University of Rhode Island

DigitalCommons@URI

Open Access Dissertations

2019

FABRICATION OF CONDUCTIVE HYDROGELS AND THEIR

APPLICATION IN NEUROPROSTHETIC INTERVENTION AND DRUG DELIVERY

Rosa Ghatee

University of Rhode Island, rosa_ghatee@yahoo.com

Follow this and additional works at: https://digitalcommons.uri.edu/oa_diss

Recommended Citation

Ghatee, Rosa, "FABRICATION OF CONDUCTIVE HYDROGELS AND THEIR APPLICATION IN

NEUROPROSTHETIC INTERVENTION AND DRUG DELIVERY" (2019). Open Access Dissertations. Paper

838.

https://digitalcommons.uri.edu/oa_diss/838

This Dissertation is brought to you for free and open access by DigitalCommons@URI. It has been accepted for inclusion in Open Access Dissertations by an authorized administrator of DigitalCommons@URI. For more information, please contact digitalcommons-group@uri.edu. 


\title{
FABRICATION OF CONDUCTIVE HYDROGELS AND \\ THEIR APPLICATION IN NEUROPROSTHETIC INTERVENTION AND DRUG DELIVERY
}

\section{BY}

ROSA GHATEE

\begin{abstract}
A DISSERTATION SUBMITTED IN PARTIAL FULFILLMENT OF THE REQUIREMENTS FOR THE DEGREE OF DOCTOR OF PHILOSOPHY

IN

CHEMICAL ENGINEERING
\end{abstract}

UNIVERSITY OF RHODE ISLAND 
DOCTOR OF PHILOSOPHY

OF

ROSA GHATEE

\section{APPROVED:}

Dissertation Committee:

Major Co-Professor

Major Co-Professor
Samantha A. Meenach

Walter Besio

Daniel Roxbury

Kunal Mankodiya

Nasser H. Zawia

DEAN OF THE GRADUATE SCHOOL

UNIVERSITY OF RHODE ISLAND 


\begin{abstract}
Electrically-responsive biomaterials such as conductive polymers and conductive hydrogels have piqued the interest of scientists for targeted payload delivery applications in recent years since generating electricity is easy. Conductive composite hydrogels are a specific class of hydrogel that can be designed to not only have mechanical properties similar to skin and soft tissue, but can also have electrical properties comparable to metals and organic semiconductors. These composite hydrogels have been considered for a wide range of technological applications. Conductive hydrogels can be fabricated to ensure that they exhibit: 1) excellent biocompatibility; 2) soft and tunable mechanical properties similar to biological tissue; 3) mixed electronic/ionic conductivity that promotes efficient signal transduction for delivering drugs to the tissue; 4) ability to create materials with well-controlled microstructure; and 5) ability to be loaded with wide range of molecular size molecules with different molecular charge; thereby making conductive hydrogels a stronger tool for targeted delivery applications.

This dissertation focuses on describing a novel method of creating hydrogel composites from the commercially available conductive polymer PEDOT (poly $(3,4-$ ethylenedioxythiophene):polystyrene sulfonate). The resulting method allows for fast production of conductive hydrogels, and does not need specific equipment. In this method of production, a wide range of mechanical properties can be achieved by altering hydrogel composition and production temperature without jeopardizing the electrical properties of the hydrogels by using conductive polymer. Additionally, this
\end{abstract}


method could be employed for fabrication of conductive composite hydrogels with a variety of polymers and cross-linkers.

Chapter two provides an overview on electrically-responsive targeted delivery depots with respect to their release mechanisms from electrical stimulation, parameters affecting drug release with electricity, and current efforts on multi-drug delivery using electrically-responsive materials.

In chapter three, we explored the production and application of pAAc-PEDOT (poly acrylic acid-poly(3,4-ethylenedioxthiophene):polystyrene sulfonate) cryogels. Our goal was to develop soft, injectable, and conducting hydrogel-based electrode materials, and to characterize their sustained mechanical and electrical properties before and after sterilization and injection. Biocompatibility, cytotoxicity, and drug delivery capability of these cryogels were also investigated. pAAc-PEDOT cryogels were made at a subfreezing temperature to generate a macroporous structure within the gels. The resulting porous hydrogels exhibited enhanced mechanical properties. The cryogels exhibited softness $(0.2-20 \mathrm{kPa})$, excellent toughness, and strain of failure, and could survive injection through 16-gauge needle. Additionally, these gels demonstrated the capability of recording alpha oscillations. Last but not least, these cryogels were found to be biocompatible and were capable of being loaded with and delivering proteins.

In chapter four, we investigated the integration of conductive hydrogels (alginatePEDOT) with tripolar concentric ring electrodes (TCREs). Our goal was to develop hydrogel-integrated electrodes with isolated channels to improve the quality of recorded signals. To this end, we fabricated alginate-PEDOT hydrogels and explored 
their mechanical and electrical properties to identify an optimized hydrogel formulation. We also investigated the channel isolation of our proposed electrode design. Furthermore, we used our novel electrode to record neural activity and compared recorded signals of hydrogel TCREs with those of conductive paste.

Chapter five provides overall conclusions for this dissertation as well as comments on potential future work. 


\section{ACKNOWLEDGMENTS}

I would like to express my sincere gratitude to my advisors Dr. Stephen Kennedy and Dr. Walter Besio for their continuous advice and support throughout my graduate study and related research; in particular for their patience, motivation, and immense knowledge. Their guidance helped me during the research and writing of this dissertation. and I could not have imagined achieving this without their support. I would also like to extend my gratitude to Dr. Samantha Meenach for stepping in as my advisor, and for her guidance, support and, insightful knowledge throughout my time in graduate school.

I would also like to thank all the staff and faculty in URI's Department of Chemical Engineering and Department of Electrical, Computer, and Biomedical Engineering for providing me with a great platform for my training and research. I also would like to specifically thank Dr. Richard Brown for granting me full access to his lab and equipment needed for the completion of my research. I would also like to thank Dr. Everett Crisman for his suggestions and help with SEM imaging. I would like to thank Dr. Geoffrey Bothun for his insightful suggestions.

Besides my advisors, I would like to thank the rest of my dissertation committee: Dr. Daniel Roxbury and Dr. Kunal Mankodiya for their insightful suggestions and participation.

I would like to extend my gratitude to all of the undergraduate students I have the pleasure of working with, including Anne Reisch, Tanner Barnes, Abed Alsassa, Justine Hayes, Jennifer Fijalkowski, Brandon Williams, and Jason Mercier. 
I also thank the CRE medical employees, Preston Steele and Joanne Hall, for their help for EEG recordings. Finally, I would like to thank my parents for their endless love and support throughout my life, and my sisters, Sara and Maryam, for always being there for me. I also thank my friends Tania, Minoo, and Anita for their help throughout completion of this research.

Funding for this work was supported through start-up funds from the University of Rhode Island's College of Engineering (Kennedy), an Early Career Development Award from the RI-INBRE (NIH/NIBMS 2P20GM103430, and an NSF EPSCoR grant (1539068). 


\section{PREFACE}

This dissertation is written in manuscript format in accordance with University of Rhode Island Graduate School Guidelines. There are five chapters included in this dissertation. The first chapter is the instruction and motivation to this work. The second chapter, entitled "Electrically-Responsive Targeted Delivery Depots" is part of an invited review paper in preparation to be submitted to "Acta Biomaterialia." The third chapter, entitled "Cryogel-Based Electronic-Tissue Interface with Soft, Highly Compressible and Tunable Mechanics" is under review in "Advanced Health Care Materials." The fourth chapter, entitled "Integration of Conductive Hydrogel into Tripolar Concentric Configuration to Improve Channel Isolation“" is prepared to be submitted to "Advanced Health Care Materials." The fifth chapter includes the conclusions and future work related to this dissertation. 


\section{TABLE OF CONTENTS}

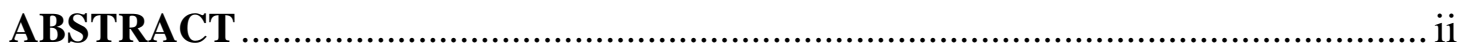

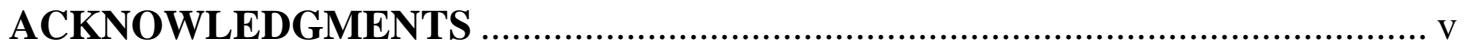

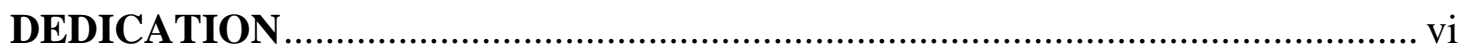

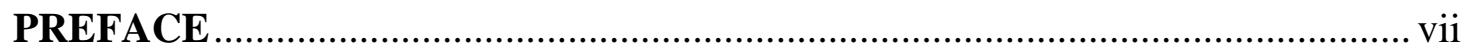

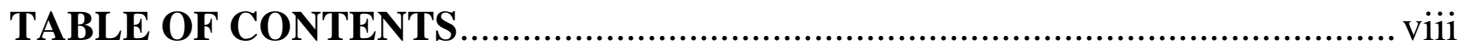

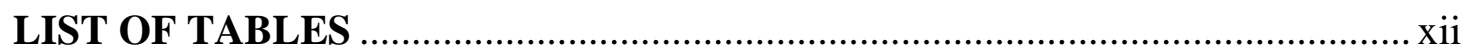

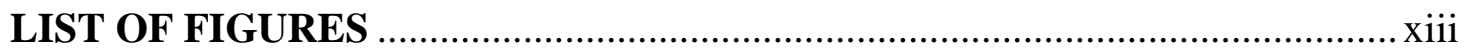

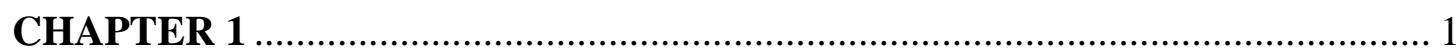

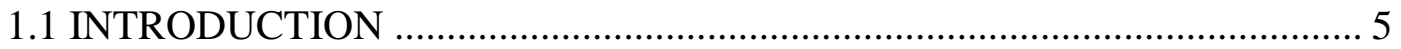

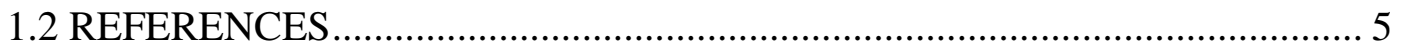

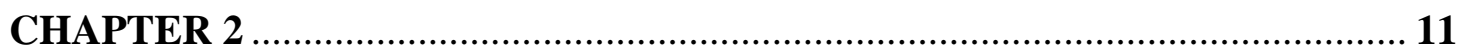

ELECTRICALLY RESPONSIVE TARGETED DELIVERY DEPOT .................. 11

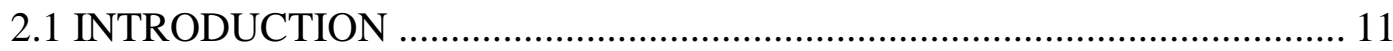

2.2 RELEASE MECHANISM FROM ELECTRICAL STIMULATION.............. 14

2.2.1 DRUG RELEASE BY PRESSURE …..................................................... 14

2.2.2 DRUG RELEASE BY MATRIX DEGREDATION .................................... 16

2.3 PARAMETERS AFFECTING DRUG RELEASE WITH ELECTRICITY ... 18

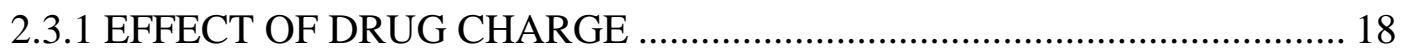

2.3.2 EFFECT OF MATRIX CONCENTRATION AND CROSS-LINKING ..... 20

2.3.3 EFFECT OF USING CONDUCTIVE MATERIALS ................................... 21

2.3.4 EFFECT OF ELECTRIC FIELD POLARITY ……………..................... 23

2.3.5 EFFECT OF ELECTRIC FIELD STRENGTH........................................... 25 


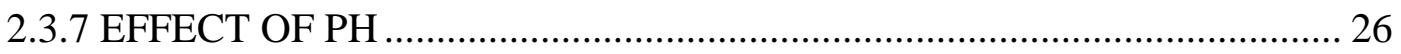

2.3.8 EFFECT OF DIRECTION OF ELECTRIC FIELD ..................................... 27

2.3.9 EFFECT OF POROSITY AND MESG SIZE ………………………......... 27

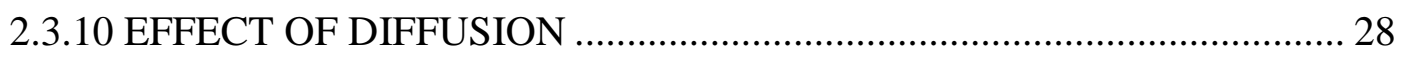

2.3.11 EFFECT OF APPLYING ELECTRIC FIELD ON TISSUE

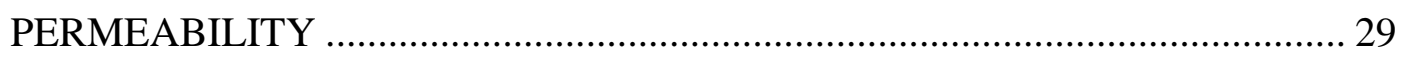

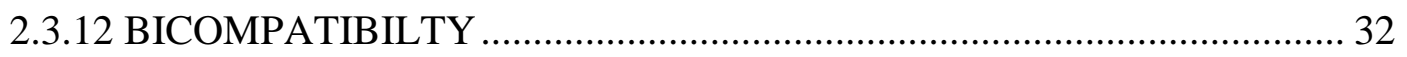

2.3.13 DISTANCE OF ELECTRODE FROM DEPOT ……………..................... 35

2.4 CURRENT EFFORTS ON MULTI-DRUG DELIVERY USING ELECTRICALLY RESPONSIVE MATERIALS ……........................................ 37

2.5 CONCLUSION AND FUTURE PERSPECTIVE.......................................... 38

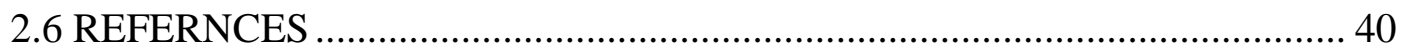

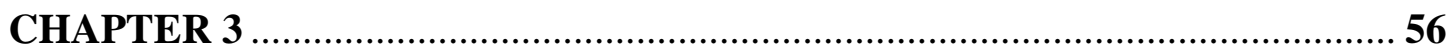

CRYOGEL-BASED ELECTRONIC-TISSUE INTERFACES WITH SOFT, HIGHLY COMPRESSIBLE AND TUNABLE MECHANICS ........................... 56

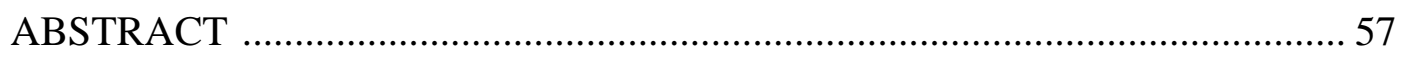

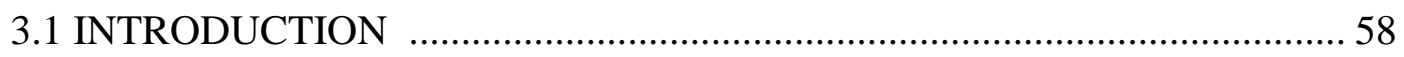

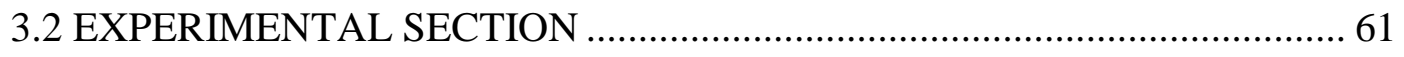

3.2.1 PEDOT/PAAC HYDROGEL AND CRYOGEL PREPARATION ............. 61

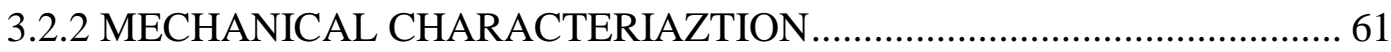

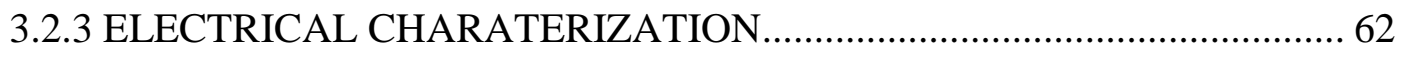

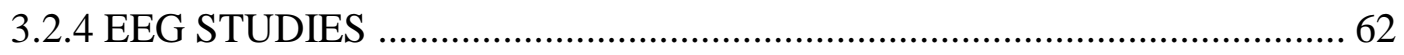




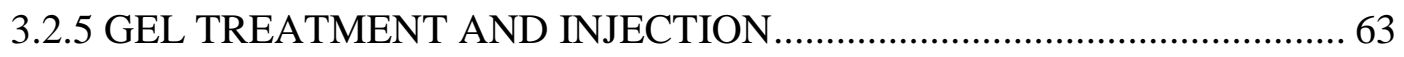

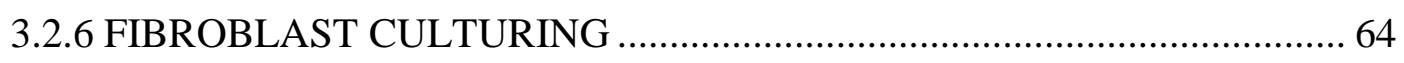

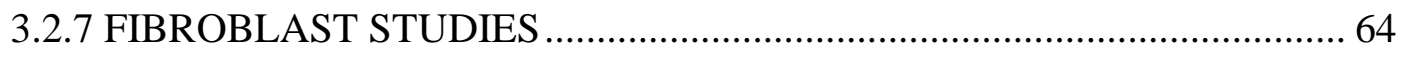

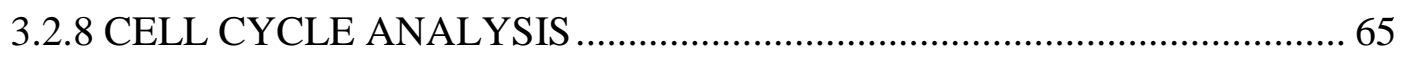

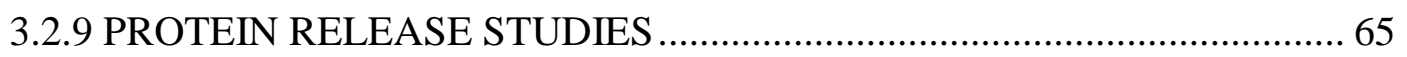

3.2.10 SAMPLE PREPARATION FOR SEM IMAGING..................................... 66

3.2.11 DATA REPRESENTATION AND STATISTICAL ANALYSIS .............. 66

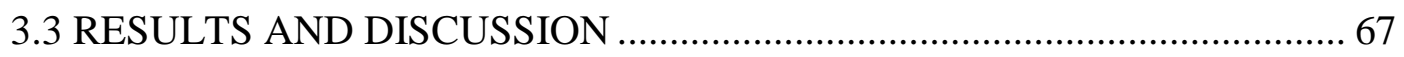

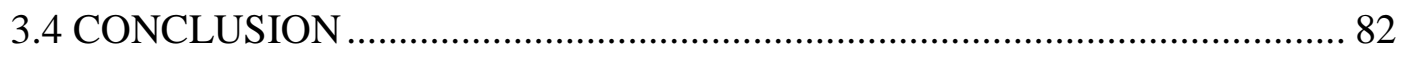

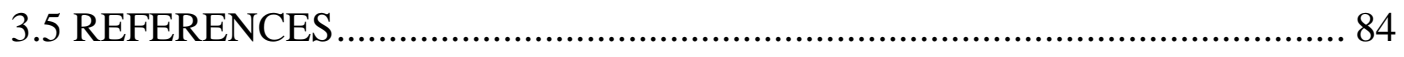

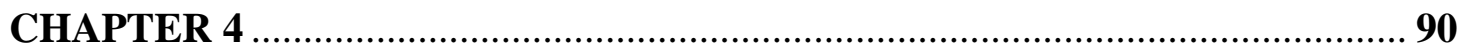

INTEGRATION OF CONDUCTIVE HYDROGEL INTO TRIPOLAR CONCENTRIC CONFIGURATION TO IMPROVE CHANNEL ISOLATION 90

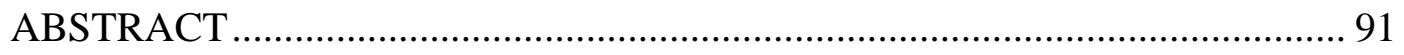

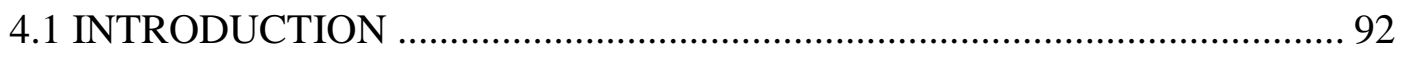

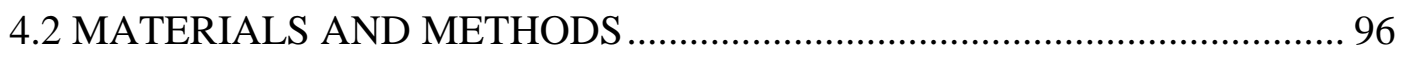

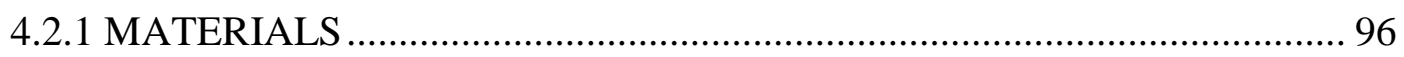

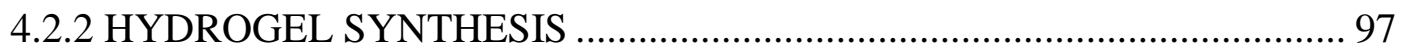

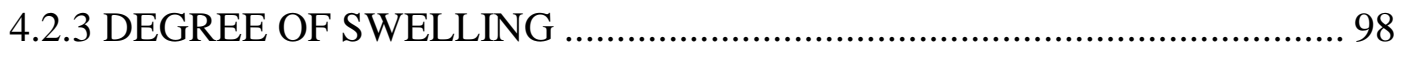

4.2.4 MECHANICAL CHARACTERIZATION................................................. 98

4.2.5 ELECTRICAL CHARACTERIZATION …………..................................... 99

4.2.6 HYDROGEL ELECTRODE FABRICATION ……………...................... 99

4.2.7 INSULATION BETWEEN CHANNELS ................................................... 101 


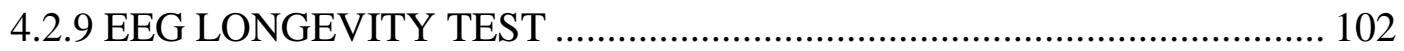

4.2.10 SIGNAL-TO-NOISE RATIO CALCULATION........................................ 102

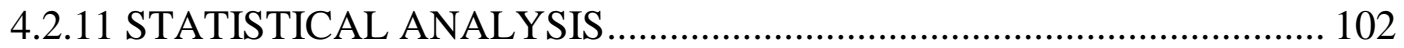

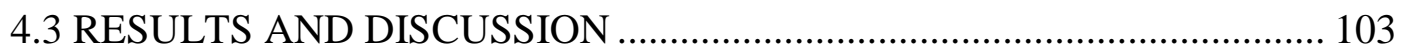

4.3.1 HYDROGEL SYNTHEIS AND DEGREE OF SWELLING …................. 103

4.3.2 MECHANICAL CHARACTERIZATION................................................. 105

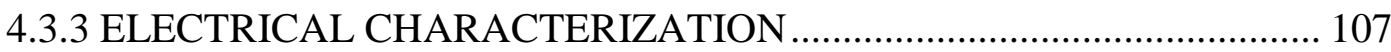

4.3.4 ISOLATION BETWEEN THE CHANNELS .......................................... 110

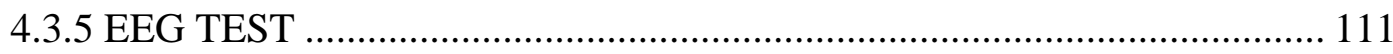

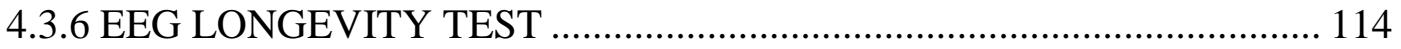

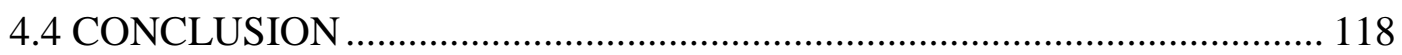

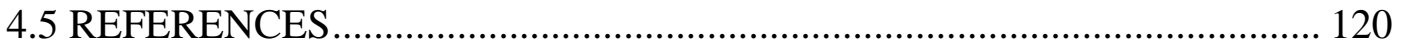

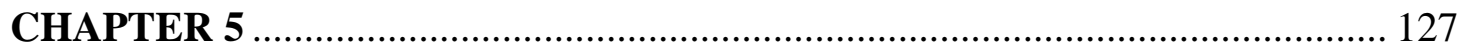

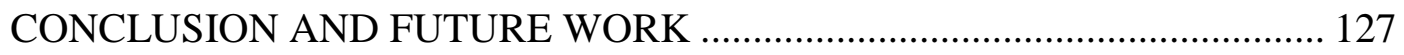

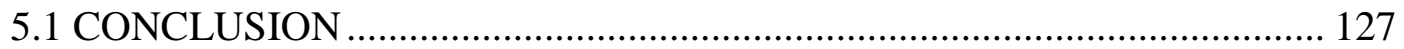

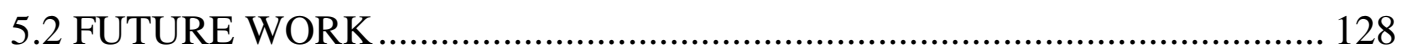

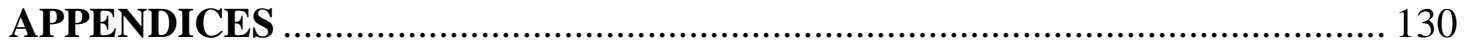

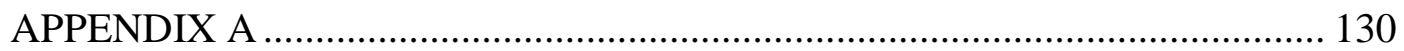

SUPPLEMENTARY MATERIALS FOR CHAPTER 3 ...................................... 130

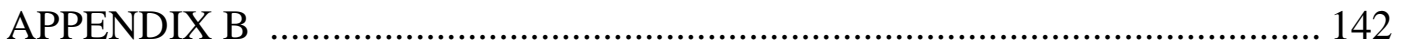

SUPPLEMENTARY MATERIALS FOR CHAPTER 4.................................. 142

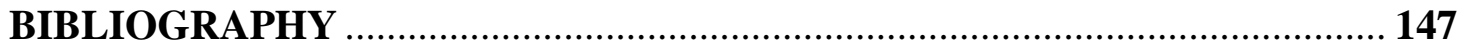




\section{LIST OF TABLES}

TABLE

PAGE

Table 4.1. Signal-to-noise ratio at $\mathrm{Oh}$ and $4 \mathrm{~h}$. signal-to-noise ratio for TCRE with Ten20 paste and $2.5 \mathrm{~mm}$ TCRHE at 0 hour and 4 hour and for $5 \mathrm{~mm}$ TCRHE at $0 \mathrm{~h}$. 


\section{LIST OF FIGURES}

FIGURE

PAGE

Figure 2.1 Hydrogel deformation that occurs when an electric field has been applied.

Figure 2.2 Matrix deform and drug releases once an electric field is applied. Representative hydrogel microstructure with (A) no electric field applied, (B) electric field applied with matrix shrinkage, (C) electric field applied with matrix swelling. 16

Figure 2.3. Schematic of hydrogel degradation and drug release upon applying electric field. (A) no electric field is applied and no drug release occurs, and (B) degradation and drug release occurs as a result of an applied electric field. 17

Figure 2.4. Surface erosion of matrix can occur upon applying an electric field. Graphs show weight loss of poly(ethyloxazolion)-poly-(methacrylic acid) complex in 0,9\% saline when $10 \mathrm{~mA}$ current is passed with (A) continuous current or (B) step-function electric current [70]. 17

Figure 2.5. Drug release rate decrease with increasing drug size. Tannic acid has the biggest size and therefore has the lowest release rate. Benzoic acid had the smallest size and hence has the highest release rate. Benzoic acid $M_{w}=122$. Folic acid $M_{w}=$ 441, tannic acid $\mathrm{M}_{\mathrm{w}}=1701$ [32]. 19 
Figure 2.6. Increasing cross-linking ratio in hydrogel decreases the rate of drug release. Amount of folic acid releases from alginate-calcium (Alg-Ca) hydrogel with 0.3, 0.7 and 1.3 cross-linking ratios [32]. 20

Figure 2.7. Increasing the amount of conductive agent enhances the drug release rate. (A) Pulsatile drug release from hydrogel upon applying electric field. Drug release rate was monitored while applying electric field for 5 minutes every 60 minutes. The pulsatile release of C-sucrose was determined for blank hydrogel and hydrogels with $0.05 \mathrm{mg} / \mathrm{ml}, 0.1 \mathrm{mg} / \mathrm{ml}$ and $0.2 \mathrm{mg} / \mathrm{ml}$ of pMWNTs (pristine multi wall carbon nanotubes). (B) C-sucrose release from hydrogel. Rate of release was calculated over time upon exposure to $10 \mathrm{~V}$ DC electric field [56]

Figure 2.8. Adding conductive agent to hydrogel enhance their mechanical properties and improves their electrical properties. (A) Stress-strain curve for an acrylic acid hydrogel with and without graphene. Increasing the amount of graphene particles in the hydrogel increases hydrogel stiffness and toughness [65]. (B) Electrical properties of poly (methacrylic) acid hydrogel improves with addition of carbon nanotubes. Where increasing carbon nanotube decreases resistance [59]. 23

Figure 2.9. Drug release in response to electrical stimulation of opposite polarity. Release of benzoic acid loaded poly(vinyl acid) hydrogel in relation to time with samples exposed to cathode or anode [33]. 24 
Figure 2.10. Drug release rate increases with increasing electric field strength. Drug release from poly(AA-co-AMPS)/carrageenan/RGO (poly (acrylic acid-co-2acrylamido-2-methyl-1-propanensulfonic acid)/carrageenan/reduced graphene oxide) hydrogels with different electric field strength. Samples was placed between parallel electrodes $500 \mathrm{~mm}$ apart $[62,68]$.

Figure 2.11. Diffusion increases with increasing mesh size and decreases with increasing cross-linking. Diffusion coefficient of benzoic acid from poly(vinyl alcohol) hydrogels in relation to mesh size and cross-linking ratio without presence of electric field [33]. 28

Figure 2.12. Cumulative release (c.r.) from hydrogel can be prevented by encapsulating drug with nanoparticles. Cumulative release is observed from hydrogel with nanoparticles of polypyrrole [81]. 29

Figure 2.13. Electrical stimulation increases porosity of the cellular membrane by generating pores. (A) Drug cannot diffuse into the cell due to drug size (no electrical stimulation). (B)Pores form in cell membrane and drug enters following electric stimulation. (C) Membrane reforms once electrical pulse is removed and drug can no longer penetrate. 31

Figure 2.14. Increasing electric field strength can enhance drug permeation through skin. Effect of current strength on permeation of Ketoprofen through skin [94]. ...... 31 
Figure 2.15. With increasing surface charge permeation increases. In vitro skin permeation profile showing ascorbyl palmitate by cathodal transport. • neutral hydrogel, $\bigcirc \quad 10 \%$ DCP (dicetylphosphate) negative lipogel, $\nabla 20 \%$ DCP

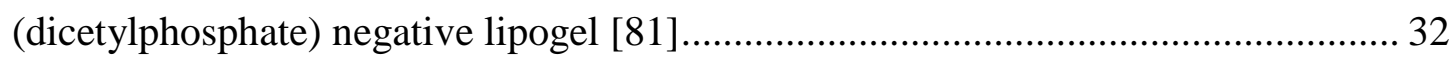

Figure 2.16. Conductive hydrogels are biocompatible, and cells maintain normal cell metabolism in direct contact with conductive hydrogels. (A) Cell proliferation of the L929 cells exposed to conductive hydrogels and TCP (mean for $n=4 \pm$ standard deviation). (B) Live/dead staining of L929 cell at successive culture periods for $\mathrm{CP} / \mathrm{OD} 1, \mathrm{CP} / \mathrm{OD} 3, \mathrm{CP} / \mathrm{OD} 5, \mathrm{C} / \mathrm{OD}$ and TCP respectively. $\mathrm{C} / \mathrm{OD}$ (chitosan/oxidize dextran), CP/OD1 (chitosan-graft-polyaniline/1 wt \% oxidize dextran), CP/OD3 (chitosan-graft- polyaniline/3 wt \% oxidize dextran), CP/OD5 (chitosan-graftpolyaniline/5 wt \% oxidize dextran) and TCP (tissue culture plate) [28] .................. 33

Figure 2.17. Conductive hydrogels are biocompatible. Figure (A) and (B) show the formation of conductive hydrogel with $1 \mathrm{wt} \%$ polypyrrole nanoparticles after subcutaneous injection in FVB mice. Black arrow indicates implants after (A) one week and (B) two weeks post implantation. Figure (C) and Figure (D) demonstrate hematoxylin and eosin (H\&E)-stained images of tissue exposed to conductive hydrogels after subcutaneous injection into FVB mouse (C) one week and (D) two weeks implantation. H\&E-stained cells could be seen in the hydrogel area [81]. ..... 34 
Figure 2.18. In vivo on-demand drug release from conductive hydrogels. In vivo fluorescent images after applying $-1.5 \mathrm{~V} / \mathrm{cm}$ to the implanted conductive hydrogel on the left side. The right side hydrogel is the control as no electric voltage was applied to it. The unit of scale of the right side of the mouse image is photons per steradian per second. (1) Before applying electric field (2) \& (3) After applying voltage for 40 seconds [81]. 36

Figure 2.19. On demand pulsatile multi-drug release from conductive hydrogels. (A) Release of fluorescein in PBS following application of $-0.5 \mathrm{~V}$ and $-1.5 \mathrm{~V}$ for 10 seconds every 5 minutes. (B) release of daunorubicin in PBS following application of $0.5 \mathrm{~V}$ for 10 second every 5 minutes [81]. 37

Figure 2.20. On demand multi-drug release from a single microchip device. The release rate calcium ions $(\Delta)$ sodium fluorescein $(\bullet)$. (Release rate unit is $\mathrm{ng} / \mathrm{min}$ ) [48]

Figure 3.1. Cryogelation of PEDOT/pAAc hydrogels result in macroporous structures with soft, tunable, and compressible mechanics. (a) Schematic detailing the cryogelation process. (b) Photograph (top) and SEM cross-section (bottom) of a 9 wt\% AAc, 1 wt\% PEGDM, 0.26 wt\% PEDOT cryogel. (c) Compressive Young's modulus (i), strain of failure (ii), and toughness (iii) vs. acrylic acid concentration for PEDOT/pAAc cryogels that were crosslinked with 1 wt $\%$ (red), 2 wt $\%$ (blue), or 5 wt $\%$ PEGDM. *, **, and $* * * *$ indicate statistically significant differences with $p$ - 
values of less than $0.05,0.01$, and 0.0001 , respectively $(\mathrm{N}=4) .+$ indicates that gels did not fail when compressed at $90 \%$ strain. Thus, strain of failure was at least $90 \%$ and hydrogel toughnesses were underestimates. 70

Figure 3.2. PEDOT/pAAc cryogels exhibited conductive electrical properties sufficient to record neural signals. (a) A schematic (i) and photo (ii) of a 9 wt $\%$ AAc, $0.26 \mathrm{wt} \%$ PEDOT, $1 \mathrm{wt} \%$ PEGDM cryogel completing an LED circuit. (b) DC conductivity vs. acrylic acid concentration for PEDOT cryogels crosslinked using 1 wt $\%$ (red), $2 \mathrm{wt} \%$ (blue), and $5 \mathrm{wt} \%$ (blue) PEGDM. (c) (i) Impedance vs. frequency for $4 \mathrm{wt} \%$ AAc, $0.26 \mathrm{wt} \%$ PEDOT cryogels crosslinked with $1 \mathrm{wt} \%$ (red) and 5wt\% (blue) PEGDM. (ii) Impedance vs. frequency for $9 \mathrm{wt} \%$ AAc, $0.26 \mathrm{wt} \%$ PEDOT cryogels crosslinked with $1 \mathrm{wt} \%$ (red) and 5wt\% (blue) PEGDM. (d) (i) Images of disc electrodes with standard Ten20 paste (blue) and a PEDOT cryogel (red). (ii, iii) Recorded neural signal vs. time (top) and power spectrum (bottom) when subject's eyes are closed (ii) and open (iii). Insets: power spectrum from 7.5 to $12.5 \mathrm{~Hz}$, highlighting alpha wave power. For all parts, n.s. indicates that no statistical significance was found $(p>0.05)$ between any groups $(\mathrm{N}=4)$.

Figure 3.3. A PEDOT/pAAc cryogel can retain its mechanical and electrical attributes after being treated in ethanol and can survive high degrees of compression. (a) Young's modulus (i) DC conductivity when measured with a $0.5 \mathrm{~V}$ signal (ii), and impedance vs. frequency (iii) for $9 \mathrm{wt} \%$ AAc, $0.26 \mathrm{wt} \%$ PEDOT, $1 \mathrm{wt} \%$ PEGDM cryogels before treatment (red) and after ethanol-treatment (blue) or autoclaving (red). 
(b) Stress vs. strain for the same cryogel when brought to and from $90 \%$ compression. Inset: photographs of the cryogel before and after compression. (c) Photographs of a 3 x $1.25 \mathrm{~mm}$ cylindrical cryogel (as formulated in parts (a) through (b)) before and after being passed through a 16-guage needle. 76

Figure 3.4. PEDOT cryogels do not disrupt fibroblast viability and cell cycle and can deliver proteins that may help reduce inflammation. (a) Schematic of in vitro viability experiments. (b) Green/red (live/dead) fluorescent images of 3T3 fibroblasts on days 1 through 7 when cells are not exposed to a gel (top) or exposed to a 9 wt\% AAc, 0.26 wt\% PEDOT, 1 wt\% PEGDM cryogel. (c) Cell density (i) and viability (ii) vs. time when cells are not exposed to a gel (dashed curve) or exposed to a the PEDOT cryogel (solid curve). (iii) Percent of fibroblasts in various stages of their cell cycle after 10 days of exposure to PEDOT cryogels (grey) vs. fibroblasts not exposed to gels (white). ** and *** indicate statically differences between cryogel exposed cells and controls with $p<0.01$ and 0.001 , respectively. n.s. indicates that no statistical differences were found $(p>0.05)$. $\mathrm{N}=4$. (d) Cumulative IL-4 release vs. time for the PEDOT cryogel after being loaded with $1 \mu \mathrm{g}$ (red) or $5 \mu \mathrm{g}$ (blue) of IL-4. $\mathrm{N}=4$.

Figure 4.1. Conductive hydrogel not only provides contact between electrode and skin but also improves isolation between channels. Comparison of electrode's contact with skin with different conductive interfaces. (A) Tripolar concentric ring electrode (TCRE). (B) Left, TCRE in contact with skin without any interface on right, Right, TCRE with conductive paste (TEN20 paste) in contact with skin. (C) Tripolar 
concentric ring hydrogel electrode (TCRHE) in contact with skin on left and schematic of conductive hydrogel on right. 94

Figure 4.2. Fabrication of tripolar concentric ring hydrogel electrode (TCRHE). (A) Tripolar concentric ring electrode (TCRE). (B) TCR mold holds both TCRE and conductive hydrogel. (i) TCR mold oblique view. (ii) TCR mold side view, (iii) TCR mold top view. (C) Schematic of fabricating TCRHE step-by-step. (i) Applying sealant on the edge of wall between rings to isolate channels and inserting TCRE. (ii) TCRE is inserted into mold and mold is ready for injection of hydrogel. (iii) Hydrogel is being injected with 22 gauge needle. (iv) TCRHE. (D) Fabrication of TCRHE stepby-step. (i) Sealant is applied on the edge of walls between rings to isolate channels and inserting TCRE. (ii) TCR mold is ready for injection of hydrogel. (iii) hydrogel is being injected with 22 gauge needle into TCR mold. (iv) TCRHE . Hydrogel mixture shown here is $2 \mathrm{w} \%$ alginate, $2.5 \mathrm{mM} \mathrm{AAD}$ and $0.26 \mathrm{wt} \%$ PEDOT. 100

Figure 4.3. Alg-PEDOT hydrogel exhibit comparably little swelling. Comparison of the degree swelling for different conductive hydrogel with different polymer (A) $\mathrm{P}(\mathrm{AAc})$ and (B) alginate as the backbone. (A) Integration of P(AAc)-PEDOT hydrogel into TCR format for (i) hydrogel with 4 wt \% P(AAc), 5 wt \% PEGDM and 0.26 wt \% PEDOT (lowest degree of swelling). (ii) hydrogel with 9 wt \% P(AAc), 1 wt \% PEGDM and 0.26 wt \% PEDOT. (iii) comparison of degree swelling for a wide range of P(AAc) (4 - 10 wt \%) and PEGDM (1, 2 and 5 wt $\%)$ with constant 0.26 wt \% PEDOT. (B) Hydrogel made from different concentration of alginate and AAD have a 
swelling degree around 1. (i) alginate-PEDOT hydrogel integrated into TCR format for hydrogel with $2 \mathrm{wt} \%$ alginate, $2.5 \mathrm{mM}$ AAD and $0.26 \mathrm{wt} \%$ PEDOT. (ii) comparison of degree of swelling for a wide range of alginate (2 - $5 \mathrm{wt} \%)$ and AAD $(2.5 \& 5 \mathrm{mM})$ with constant $0.26 \mathrm{wt} \%$ PEDOT. $\mathrm{N}=4$ for all parts. 104

Figure 4.4. Hydrogel mechanics are tunable while degree of swelling remains around 1. Different hydrogel formulations made from alginate (2- $5 \mathrm{wt} \%)$ and crosslinked with AAD $(2.5 \& 5 \mathrm{mM})$ with constant $0.26 \mathrm{wt} \%$ PEDOT. Mechanical characteristics of hydrogels including (A) Young's modulus, (B) toughness, and (C) strain of failure. $\mathrm{N}=4$ for all parts. 106

Figure 4.5. Hydrogel electrical properties are independent of hydrogel formulation. (A). IV curve for different formulation of hydrogel made from alginate (2- $5 \mathrm{wt} \%$ ) and crosslinked with AAD (2.5 \& $5 \mathrm{mM})$ with constant 0.26 wt\% PEDOT. 8, 4, 2, 1.5, $1.5,-2,-4$ and $8 \mathrm{v}$ voltages were applied, and currents were read. (B). Impedance for different formulation of hydrogel made from alginate (2- $5 \mathrm{wt} \%)$ and crosslinked with AAD $(2.5 \& 5 \mathrm{mM})$ with constant $0.26 \mathrm{wt} \%$ PEDOT. Frequency was applied from 10 to $10^{5} \mathrm{~Hz}$ at $10 \mathrm{mv}$ voltage. (C). Conductivities for different hydrogel formulation made from alginate $(2-5 \mathrm{wt} \%)$ and crosslinked with AAD $(2.5 \& 5$ wt $\%)$ with constant 0.26 wt $\%$ PEDOT at (i) $\mathrm{V}=1.5 \mathrm{v}$, (ii) $\mathrm{V}=2 \mathrm{v}$, and (iv) $\mathrm{V}=8 \mathrm{v}$. For all parts $\mathrm{N}=4$ and n.s. indicates that no statistically significant difference was found ( $\mathrm{p}>$ $0.05)$. 109 
Figure 4.6. TCRHE exhibit much better isolation between channels in comparison to conductive paste, where high impedance between channels indicate isolation. (A) Isolation between middle ring to center disc. Red for TCRE with Ten20 paste and blue for $2.5 \mathrm{~mm}$ TCR hydrogel electrode (2.5 mm TCRHE) (2 wt \% alginate, $2.5 \mathrm{mM}$ AAD and 0.26 wt $\%$ PEDOT). (B) Isolation between center disc to outer ring. Red for TCRE with Ten20 paste and blue for $2.5 \mathrm{~mm}$ TCR hydrogel electrode $(2.5 \mathrm{~mm}$ TCRHE) (2 wt \% alginate, $2.5 \mathrm{mM}$ AAD and $0.26 \mathrm{wt} \%$ PEDOT). (C) Isolation between outer ring to middle ring. Red for TCRE with Ten20 paste and blue for 2.5 mm TCR hydrogel electrode (2.5 mm TCRHE)) (2 wt \% alginate, $2.5 \mathrm{mM}$ AAD and 0.26 wt \% PEDOT). Frequency was applied from 10 to $1000 \mathrm{~Hz}$ at $10 \mathrm{mv}$ voltage.

Figure 4.7. TCRHEs have higher amplitude and significantly improves power spectrum in comparison to TCRE with Ten20 paste. (A) EEG recording from occipital lobe using Ten20 paste (red) compared to hydrogel (2.5 $\mathrm{mm}$ lead electrode (blue)) while subject eyes were closed. Inset: zoom-in EEG recording while eyes were closed. (B). Power spectrum from occipital lobe using TEN20 paste (red) compared to hydrogel (2.5 mm electrode lead (blue)) while subject eyes were closed. Inset: zoomin power spectra, highlighting the recording of alpha waves $(8-13 \mathrm{~Hz})$ only with eyes closed. C. EEG recording from occipital lobe using TEN20 paste (red) compared to hydrogel (2.5 mm lead electrode (blue)) while subject eyes were opened. Alfa wave was vanished as soon as eyes were opened. (D). Power spectrum from occipital lobe using TEN20 paste (red) compared to hydrogel (2.5 mm lead electrode (blue)) while 
subject eyes were open. Alfa wave was disappeared as soon as eyes were opened. For all parts, $\mathrm{N}=4$ at 0 hour. * indicates p-values $<0.05$.

Figure 4.8. TCRHEs have higher amplitude and significantly improves power spectrum comparing to TCRE with Ten20 paste. (A) EEG recording from occipital lobe using TEN20 paste (red) compared to hydrogel $2.5 \mathrm{~mm}$ lead electrode (blue) while subject eyes were closed. Inset: zoom-in EEG recording while eyes were closed. (B). Power spectrum from occipital lobe using TEN20 paste (red) compared to hydrogel $2.5 \mathrm{~mm}$ electrode lead (blue) while subject eyes were closed. Inset: zoom-in power spectra, highlighting the recording of alpha waves $(8-13 \mathrm{~Hz})$ only with eyes closed. (C) EEG recording from occipital lobe using TEN20 paste (red) compared to hydrogel $2.5 \mathrm{~mm}$ lead electrode (blue), while subject eyes were opened. Alfa wave was disappeared as soon as eyes opened. (D) Power spectrum from occipital lobe using TEN20 paste (red) compared to hydrogel $2.5 \mathrm{~mm}$ lead electrode (blue) while subject eyes were open. Alfa wave was gone as soon as eyes were opened. For all parts, $\mathrm{N}=4$ at 4 hours. $*$ indicates p-values $<0.05, * *$ indicates p-values $<0.01 \ldots 115$

Figure 4.9. TCRHE had higher power spectrum comparing to TCRE with Ten20 paste over time. 8 - $13 \mathrm{~Hz}$ (alpha wave) while subject's eyes were closed. $\mathrm{N}=4$ for all parts. $*$ indicates $\mathrm{p}$-values $<0.05, * *$ indicates $\mathrm{p}$-values $<0.01$, *** indicates $\mathrm{p}$-values $<$ 0.001 and $* * * *$ indicates p-values $<0.0001$ 117

Figure A1. Nanoporous PEDOT/pAAc hydrogels exhibit soft and tunable mechanics. 
(a) Compressive modulus vs. acrylic acid concentration for gels crosslinked with 1 wt\% (red), 2 wt\% (blue), and 5 wt\% (green) PEGDM. "No gelation" indicates that there was not enough polymer and crosslinker to form a 3D gel structure and therefore no measurement could be taken. (b) Table summarizing statistically significant differences of moduli between different hydrogel formulations. $\mathrm{N}=4$

Figure A2. The electrical properties of these hydrogels are not significantly impacted by acrylic acid and PEGDM crosslinking concentration. (a) I-V curves for gels composed $0.26 \mathrm{wt} \%$ PEDOT and (i) 4 and $7 \mathrm{wt} \%$ AAc, (ii) $9 \mathrm{wt} \%$ AAc, and (iii) 10 wt $\%$ AAc. (b) Computed DC electrical conductivity for various $0.26 \mathrm{wt} \%$ PEDOT hydrogel formulations when excited using (i) $0.5 \mathrm{~V}$, (ii) $2 \mathrm{~V}$, (iii) $4 \mathrm{~V}$, and (iv) $8 \mathrm{~V}$. n.s. indicates no statistically significant differences. $\mathrm{N}=4$. Note that some gel formulations lacked sufficient polymer and crosslinker to form stable gels. 132

Figure A3. Many hydrogel formulations exhibit high strains of failure and toughness. Strain of failure (a) and toughness (b) vs. acrylic acid concentration for gels crosslinked with 1 wt\% (red), 2 wt\% (blue), and 5 wt\% (green) PEGDM. "No gelation" indicates that there was not enough polymer and crosslinker to form a 3D gel structure and therefore no measurement could be taken. For all parts, $\mathrm{N}=4$. In part (a) the dashed line at $90 \%$ indicates that compression tests never exceeded $90 \%$ as to not risk damaging the equipment, though no gels reached $90 \%$ compression due to failure at lower strains 
Figure A4. Nanoporous PEDOT/pAAc gel formulations swelled well beyond their original volumes after casting. Ratio of swollen volume over ordinally cast volume for PEDOT/pAAc gels vs. acrylic acid concentration for gels crosslinked with $1 \mathrm{wt} \%$ (red), $2 \mathrm{wt} \%$ (blue), and $5 \mathrm{wt} \%$ (green) PEGDM. "No gelation" indicates that there was not enough polymer and crosslinker to form a 3D gel structure and therefore no measurement could be taken. For all parts, $\mathrm{N}=4$ 134

Figure A5. Nanoporous PEDOT/pAAc gel cannot survive the strains associated with injection. (a) Compressive stress vs. strain of for a $5 \times 5 \mathrm{~cm}$ cylindrical $9 \mathrm{wt} \%$ AAc, 0.26 wt $\%$ PEDOT, 1 wt $\%$ PEGDM hydrogel, until failure (b) Photographs of a $3 \mathrm{x}$ $1.25 \mathrm{~mm}$ cylindrical hydrogel (formulated as in part (a)) before and after injection through a 16-guage needle 135

Figure A6. PEDOT cryogels exhibited similar DC conductivities over a wide range of gel formulations. DC conductivity vs. gel formulation for cryogels containing 0.26 wt\% PEDOT when excited at $0.5 \mathrm{~V}$ (a), $2 \mathrm{~V}$ (b), $4 \mathrm{~V}$ (c), and $8 \mathrm{~V}$ (d). n.s. indicates that no statistically significant differences were found $(\mathrm{N}=4)$.

Figure A7. Nyquist plots for a range of PEDOT cryogel formulations exhibit similar characteristics. (a) Real vs. imaginary component of impedance for 4 wt $\%$ AAc PEDOT cryogels crosslinked with 1 wt $\%$ (red) and 5 wt $\%$ (blue) PEGDM. (b) Real vs. imaginar component of impedance for $9 \mathrm{wt} \%$ AAc PEDOT cryogels crosslinked with $1 \mathrm{wt} \%$ (green) and $5 \mathrm{wt} \%$ (black) PEGDM. $\mathrm{N}=4$. 137 
Figure A8. Exposure to PEDOT cryogels did not visually impact the skin. Subject forearm before gel placement (top), after 1 hour of exposure when gels (middle), and immediately after gel removal (bottom). PAAc-PEDOT cryogel was made at $9 \mathrm{wt} \%$ AAc, $1 \mathrm{wt} \%$ PEGDM, and $0.26 \mathrm{wt} \%$ PEDOT. The PAAc cryogel was made at $9 \mathrm{wt} \%$ AAc, $1 \mathrm{wt} \%$ PEGDM, and $0 \mathrm{wt} \%$ PEDOT 138

Figure A9. Ethanol-treatment and autoclaving PEDOT cryogels do not statistically alter electrical conductivity. DC electrical conductivity computed at $2 \mathrm{~V}$ (a), $4 \mathrm{~V}$ (b), and $8 \mathrm{~V}$ (c) for PEDOT cryogels (9 wt $\%$ AAc, $1 \mathrm{wt} \%$ PEGDM, and $0.26 \mathrm{wt} \%$ PEDOT) before treatment (red), after ethanol-treatment (blue), or after autoclaving (green). n.s. indicates that no statistically significant differences were observed $(\mathrm{N}=$ 4). 139

Figure A10. PEDOT cryogel-exposed fibroblasts do not exhibit different cell cycles as compared to controls. Histograms of propidium iodide (PI) intensity for fibroblasts after 10 days when not exposed to PEDOT cryogels (top row) compared to fibroblasts exposed to PEDOT cryogels (bottom row).

Figure A11. PEDOT/pAAc cryogel DC resistance decreases as the gel is compressed. DC resistance vs. compressive strain for 9 wt $\%$ AAc, 0.26 wt $\%$ PEDOT, 1 wt \% PEGDM cryogels. $\mathrm{N}=4$. 141

Figure B1. TCR hydrogel electrode have a much better isolation between channels. 
High impedance between channels indicates isolation. (A) Isolation between middle ring to center disc. Red for TCRE with Ten20 paste, blue for $2.5 \mathrm{~mm}$ TCR hydrogel electrode (2.5 mm TCRHE) and green $5 \mathrm{~mm}$ TCR hydrogel electrode ( $5 \mathrm{~mm}$ TCRHE) (2 wt \% alginate, $2.5 \mathrm{mM}$ AAD and $0.26 \mathrm{wt} \%$ PEDOT). (B) Isolation between center disc to outer ring. Red for TCRE with Ten20 paste, blue for $2.5 \mathrm{~mm}$ TCR hydrogel electrode (2.5 mm TCRHE) and green $5 \mathrm{~mm}$ TCR hydrogel electrode ( $5 \mathrm{~mm}$ TCRHE) (2 wt \% alginate, $2.5 \mathrm{mM}$ AAD and $0.26 \mathrm{wt} \%$ PEDOT). (C) Isolation between outer ring to middle ring. Red for TCRE with Ten20 paste, blue for $2.5 \mathrm{~mm}$ TCR hydrogel electrode (2.5 mm TCRHE) and green $5 \mathrm{~mm}$ TCR hydrogel electrode ( $5 \mathrm{~mm}$ TCRHE) ( $2 \mathrm{wt} \%$ alginate, $2.5 \mathrm{mM}$ AAD and $0.26 \mathrm{wt} \%$ PEDOT). $\mathrm{N}=4$ for all parts

Figure B2. TCR hydrogel electrode have higher amplitude and significantly improves power spectrum comparing to TCRE with Ten20 paste. (A) EEG recording from occipital lobe using TEN20 paste (red) compared to hydrogel (2.5 mm lead electrode (blue) and $5 \mathrm{~mm}$ lead electrode) while subject eyes were closed. (i) Inset: zoom-in EEG recording while eyes were closed. (B) Power spectrum from occipital lobe using TEN20 paste (red) compared to hydrogel (2.5 mm electrode lead (blue) and $5 \mathrm{~mm}$ lead electrode (green)) while subject eyes were closed. (i) Inset: zoom-in power spectra, highlighting the recording of alpha waves $(8-13 \mathrm{~Hz})$ while eyes were closed. (C) EEG recording from occipital lobe using TEN20 paste (red) compared to hydrogel $(2.5 \mathrm{~mm}$ lead electrode (blue) and $5 \mathrm{~mm}$ lead electrode) while subject eyes were opened. Alfa wave was gone as soon as eyes were opened. (D) Power spectrum from occipital lobe using TEN20 paste (red) compared to hydrogel $(2.5 \mathrm{~mm}$ lead electrode (blue) and 5 
mm lead electrode(green)) while subject eyes were open. Alfa wave was gone as soon as eyes were opened. For all parts, $\mathrm{N}=4$ at 0 hour. ............................................. 144 


\section{CHAPTER 1}

\section{Introduction and Motivation}

Neuroprosthetic devices have been developed in order to return function, or to reduce symptoms exhibited by patients suffering from neurological diseases [1]. For instance, electrical therapies such as cochlear implants [2, 3] and deep brain stimulation $[4,5]$ are promising strategies in treating patients with neurological diseases. While promising, these strategies are not without limitations [1]. For example, the strategies require sustained tissue-electrode interface over time [6] and the electrodes must minimize overpotential at the tissue-electrode interface, and maintain a conductive path between the electrode and the tissue being stimulated. Traditionally, metallic electrodes have been used for stimulation of neural tissue. Despite their outstanding electrical properties over short periods of time, metallic electrodes do not maintain these properties over long periods of time [7]. In addition, introduction of these electrodes to patients requires invasive surgery that often ruptures neurons and blood vessels and activates a foreign body response at the site of electrode tissue interface [1]. Additionally, the mismatch between the mechanical properties of the electrode and tissue can cause shear stress at the interface, which can in turn result in prolonged inflammation at the site of electrode, and eventually the loss of connection between the electrode and surrounding tissue [8-11]. These constraints

pose limitations for the application of the commonly-used electrodes over extended periods of time. 
Electroencephalography (EEG) is an affordable, non-invasive technique used to measure electrical impulses generated by brain activity. The EEG has excellent temporal resolution, which is ideal for diagnosing neurological diseases. For example, EEG is currently used for diagnosing diseases such as epilepsy and head trauma [12, 13]. Furthermore, it is extensively used in neuro-rehabilitation applications such as in the brain-computer interface to help control the movement of prosthetic limbs in patients with paralysis. [14-17]. However, while EEG has an excellent temporal resolution, it has a limited spatial resolution. Because of the aforementioned benefits offered by EEG, several methods, such as surface Laplacian, have been designed to improve the spatial resolution of EEG. Surface Laplacian methods not only enhance spatial selectivity, but also attenuate universal noise and improve signal-to-noise ratio [16, 18-23]. Several electrodes have been designed based on surface Laplacian theory, which have a center disc and one or more rings around the center disc [18, 24]. These electrodes are often made of metals, which require electrical connections with the scalp. To successfully make a connection between electrodes and a patient's skin, conductive paste is typically used [25]. While conductive paste significantly improves the electrode-skin interface, it also partially electrically shorts the channels of electrodes and affects the quality of recorded signals. Moreover, conductive paste dries out over time, negatively affecting the quality of signal and increasing noise over time. These obstacles limit the application of conductive paste where EEG spatiotemporal resolution over time is vital. Given the constraints of EEG-based neuroprosthetic devices with respect to their current methods and the limitations of their applicability, we aimed to develop a novel biomaterial system that can provide an 
effective electrical connection between the scalp and electrode, be formed into a desired shape for the given application, and retain its integrity for long periods of time.

To address the aforementioned issues, we first aimed to develop an improved conductive interface between tissue and the electrode using conductive hydrogels. Hydrogels are soft materials made from cross-linked hydrophilic polymeric networks [26]. Although hydrogels themselves are insoluble in water, they can preserve a large fraction of water within their network for prolonged periods of time while successfully retaining their 3D structure [27]. Conductive polymer hydrogels are a unique class of hydrogels, which not only have similar mechanical properties to tissue [28], but also have similar electrical properties to metals and inorganic semiconductors [29]. There are currently several conductive polymers available, which have been widely used for fabricating conductive hydrogels [30]. It has been demonstrated that the conductive polymer poly(3-4, ethylenedioxythiophene) (PEDOT) provides better conductivity and stability in oxidized environments than other widely used conductive polymers [3136]. Moreover, PEDOT is biocompatible [37]. Thus, for the purpose of this study, PEDOT was used as the conductive component of the electrically conductive hydrogel.

PEDOT is a conjugated polymer with an outstanding chemical stability [30, 38] owing to the fact that the EDOT monomer has only two reactive hydrogens, which means that it is unlikely to have chemical defects in the PEDOT chain [39]. PEDOT is commonly used in its oxidative state where some of electrons are removed from main chain, generating positive carriers, and thus makes the polymer electrically conductive [40]. Although PEDOT is not soluble in water, it is possible to form an aqueous 
suspension by doping it with different types of anions such as poly(styrene sulfonate) (PSS) [39]. PEDOT and its water soluble PEDOT:PSS are the most commonly used conducting polymers [38].

For the resulting injectable conductive hydrogel systems, we employed a shape memory technique during hydrogel synthesis by fabricating the hydrogels at a subfreezing temperature, which results in hydrogels with interconnected macropores [41]. The ability of the hydrogels to maintain their shape can allow for minimally invasive implantation of the gels at the application site [42]. The novel method of conductive hydrogel fabrication used in the described studies utilized a commercially available conductive polymer, which allows for fast production without the need for special equipment. In addition, the resulting synthesis methods could be possibly used to fabricate conductive hydrogels with variety of polymer types for a wide variety of applications.

While PEDOT hydrogels have been created previously, they have yet to be employed as a means to enhance the spatial resolution of multi-channel electrode systems by providing soft, 3D conducting structures that retain their shape and moisture over prolonged periods of time. Thus, the work proposed here is a novel step forward in the context of contemporary technologies related to neuroprosthetics.

Overall, we hypothesized that by integrating the conductive polymer PEDOT within a hydrogel network and designing the morphology of the resulting conductive hydrogels based on the given application, that a sustained conductive interface between the electrode and tissue can be achieved. 


\section{References}

[1] J. Leach, A.K. Achyuta, S. Murthy, Bridging the divide between neuroprosthetic design, tissue engineering and neurobiology, Frontiers in Neuroengineering 2(18) (2010).

[2] F.G. Zeng, S. Rebscher, W. Harrison, X. Sun, H. Feng, Cochlear implants: system design, integration, and evaluation, IEEE Rev Biomed Eng 1 (2008) 115-42.

[3] B.S. Wilson, M.F. Dorman, Cochlear implants: a remarkable past and a brilliant future, Hear Res 242(1-2) (2008) 3-21.

[4] N.R. Williams, M.S. Okun, Deep brain stimulation (DBS) at the interface of neurology and psychiatry, J Clin Invest 123(11) (2013) 4546-56.

[5] M.K. Lyons, Deep brain stimulation: current and future clinical applications, Mayo Clin Proc 86(7) (2011) 662-72.

[6] R.T. Hassarati, W.F. Dueck, C. Tasche, P.M. Carter, L.A. Poole-Warren, R.A. Green, Improving cochlear implant properties through conductive hydrogel coatings, IEEE Trans Neural Syst Rehabil Eng 22(2) (2014) 411-8.

[7] R.A. Green, C.M. Williams, N.H. Lovell, L.A. Poole-Warren, Novel neural interface for implant electrodes: improving electroactivity of polypyrrole through MWNT incorporation, J Mater Sci Mater Med 19(4) (2008) 1625-9.

[8] P.J. Rousche, R.A. Normann, Chronic intracortical microstimulation (ICMS) of cat sensory cortex using the Utah intracortical electrode array, IEEE Transactions on Rehabilitation Engineering 7(1) (1999) 56-68. 
[9] P.J. Rousche, R.A. Normann, Chronic recording capability of the Utah Intracortical Electrode Array in cat sensory cortex, Journal of Neuroscience Methods 82(1) (1998) 1-15.

[10] R.A. Green, H. Toor, C. Dodds, N.H. Lovell, Variation in Performance of Platinum Electrodes with Size and Surface Roughness, Sensors and Materials 24(4) (2012) 165-180.

[11] M. Asplund, T. Nyberg, O. Inganäs, Electroactive polymers for neural interfaces, Polymer Chemistry 1(9) (2010).

[12] C.S. Williams, M.C. Spitz, J.F. Foley, L.K. Weaver, A.S. Lindblad, M.R. Wierzbicki, Baseline EEG abnormalities in mild traumatic brain injury from the BIMA study, Undersea Hyperb Med 43(5) (2016) 521-530.

[13] B.L. Edlow, C. Chatelle, C.A. Spencer, C.J. Chu, Y.G. Bodien, K.L. O'Connor, R.E. Hirschberg, L.R. Hochberg, J.T. Giacino, E.S. Rosenthal, O. Wu, Early detection of consciousness in patients with acute severe traumatic brain injury, Brain 140(9) (2017) 2399-2414.

[14] M. Teplan, Fundamentals of EEG measurements, MEASUREMENT SCIENTIFIC REVIEW 2 (2002).

[15] S.A. Park, H.J. Hwang, J.H. Lim, J.H. Choi, H.K. Jung, C.H. Im, Evaluation of feature extraction methods for EEG-based brain-computer interfaces in terms of robustness to slight changes in electrode locations, Med Biol Eng Comput 51(5) (2013) 571-9.

[16] D.J. McFarland, The advantages of the surface Laplacian in brain-computer interface research, Int J Psychophysiol 97(3) (2015) 271-6. 
[17] D.B. Salisbury, T.D. Parsons, K.R. Monden, Z. Trost, S.J. Driver, Braincomputer interface for individuals after spinal cord injury, Rehabil Psychol 61(4) (2016) 435-441.

[18] W. Besio, R. Aakula, K. Koka, W. Dai, Development of a Tri-polar Concentric Ring Electrode for Acquiring Accurate Laplacian Body Surface Potentials, Annals of Biomedical Engineering 34(3) (2006) 426-435.

[19] W.G. Besio, C.C. Lu, P.P. Tarjan, A feasibility study for body surface cardiac propagation maps of humans from Laplacian moments of activation, Electromagnetics 21(7-8) (2001) 621-632.

[20] K. Koka, W.G. Besio, Improvement of spatial selectivity and decrease of mutual information of tri-polar concentric ring electrodes, J Neurosci Methods 165(2) (2007) 216-22.

[21] V. Soundarajan, W.G. Besio, Simulated comparison of disc and concentric electrode maps during atrial arrhythmias, IJBEM 7(1) (2005) 217-220.

[22] W.G. Besio, K. Koka, R. Aakula, W. Dai, Tri-polar concentric ring electrode development for laplacian electroencephalography, Ieee T Bio-Med Eng 53(5) (2006) 926-33.

[23] W. Besio, T. Chen, Tripolar Laplacian electrocardiogram and moment of activation isochronal mapping, Physiol Meas 28(5) (2007) 515-529.

[24] D. Farina, C. Cescon, Concentric-ring electrode systems for noninvasive detection of single motor unit activity, Ieee T Bio-Med Eng 48(11) (2001) 1326-34. 
[25] P. Tallgren, S. Vanhatalo, K. Kaila, J. Voipio, Evaluation of commercially available electrodes and gels for recording of slow EEG potentials, Clin Neurophysiol 116(4) (2005) 799-806.

[26] L. Pan, G. Yu, D. Zhai, H.R. Lee, W. Zhao, N. Liu, H. Wang, B.C. Tee, Y. Shi, Y. Cui, Z. Bao, Hierarchical nanostructured conducting polymer hydrogel with high electrochemical activity, Proc Natl Acad Sci U S A 109(24) (2012) 928792.

[27] S. Naficy, J.M. Razal, G.M. Spinks, G.G. Wallace, P.G. Whitten, Electrically Conductive, Tough Hydrogels with pH Sensitivity, Chemistry of Materials 24(17) (2012) 3425-3433.

[28] U.A. Aregueta-Robles, A.J. Woolley, L.A. Poole-Warren, N.H. Lovell, R.A. Green, Organic electrode coatings for next-generation neural interfaces, Frontiers in Neuroengineering 7(15) (2014).

[29] Y. Zhao, B. Liu, L. Pan, G. Yu, 3D nanostructured conductive polymer hydrogels for high-performance electrochemical devices, Energy \& Environmental Science 6(10) (2013).

[30] N.K. Guimard, N. Gomez, C.E. Schmidt, Conducting polymers in biomedical engineering, Progress in Polymer Science 32(8-9) (2007) 876-921.

[31] M. Javadi, Q. Gu, S. Naficy, S. Farajikhah, J.M. Crook, G.G. Wallace, S. Beirne, S.E. Moulton, Conductive Tough Hydrogel for Bioapplications, Macromol Biosci 18(2) (2018). 
[32] G.L. Mario Cheong, K.S. Lim, A. Jakubowicz, P.J. Martens, L.A. Poole-Warren, R.A. Green, Conductive hydrogels with tailored bioactivity for implantable electrode coatings, Acta Biomater 10(3) (2014) 1216-26.

[33] M.R. Abidian, D.H. Kim, D.C. Martin, Conducting-Polymer Nanotubes for Controlled Drug Release, Adv Mater 18(4) (2006) 405-409.

[34] M.R. Abidian, D.C. Martin, Experimental and theoretical characterization of implantable neural microelectrodes modified with conducting polymer nanotubes, Biomaterials 29(9) (2008) 1273-83.

[35] P. Huang, W. Chen, L. Yan, An inorganic-organic double network hydrogel of graphene and polymer, Nanoscale 5(13) (2013) 6034-9.

[36] S. Sekine, Y. Ido, T. Miyake, K. Nagamine, M. Nishizawa, Conducting polymer electrodes printed on hydrogel, J Am Chem Soc 132(38) (2010) 13174-5.

[37] W. Cho, J. Wu, B.S. Shim, W.F. Kuan, S.E. Mastroianni, W.S. Young, C.C. Kuo, T.H. Epps, 3rd, D.C. Martin, Synthesis and characterization of bicontinuous cubic poly(3,4-ethylene dioxythiophene) gyroid (PEDOT GYR) gels, Phys Chem Chem Phys 17(7) (2015) 5115-23.

[38] A. Patra, V. Agrawal, R. Bhargav, Shahjad, D. Bhardwaj, S. Chand, Y. Sheynin, M. Bendikov, Metal Free Conducting PEDOS, PEDOT, and Their Analogues via an Unusual Bromine-Catalyzed Polymerization, Macromolecules 48(24) (2015) 8760-8764.

[39] D.C. Martin, J. Wu, C.M. Shaw, Z. King, S.A. Spanninga, S. Richardson-Burns, J. Hendricks, J. Yang, The Morphology of Poly(3,4-Ethylenedioxythiophene), Polymer Reviews 50(3) (2010) 340-384. 
[40] T. Yamamoto, Molecular assembly and properties of polythiophenes, NPG Asia Materials 2(2) (2010) 54-60.

[41] A. Kumar, A. Srivastava, Cell separation using cryogel-based affinity chromatography, Nat Protoc 5(11) (2010) 1737-47.

[42] L. Wang, J. Shansky, C. Borselli, D. Mooney, H. Vandenburgh, Design and fabrication of a biodegradable, covalently crosslinked shape-memory alginate scaffold for cell and growth factor delivery, Tissue Eng Part A 18(19-20) (2012) 2000-7. 


\section{CHAPTER 2}

\section{Electrically-Responsive Targeted Delivery Depots}

\subsection{Introduction}

Electrical stimulation can be used for a variety of treatments such as pain management [1-4], neuromuscular dysfunction [5], strength training [6], improving local blood flow [7], motor control and retardation of muscle atrophy [2, 8, 9], improving joint mobility [10], tissue repair [9] and wound healing [11-13], and acute and chronic edema [14-17]. Electrical stimulation has also been used for drug delivery [18], brain computer interface (BCI) [5], deep brain stimulation [19], cochlear implants [20, 21], transcranial direct current stimulation (tDCS) [7], etc.

Stimuli-responsive biomaterials have garnered great interest in recent years for their use in drug delivery applications [25]. Although many delivery systems have been developed that can respond to electromagnetic fields, light, ultrasound, or $\mathrm{pH}$ [23-25], activating these systems requires the use of large and specialized devices. On the other hand, generating electric stimuli typically does not require large or specialized equipment [26, 27]. Moreover, designing and miniaturizing the type of equipment required for applying electrical stimulation is an advantage [28] of using electrical stimulation for drug delivery applications. Hydrogels serve as drug reservoirs where drugs can be physically entrapped within hydrogel network [29, 30], have electrostatic interactions with hydrogel network [31-33], or bind with conductive polymers [34-36] and nanoparticles [27,31,37, 38]. 
Electrically stimulated drug delivery systems are platforms that allow localized and on-demand drug release for a variety of applications [39]. For example, electrical pulses can enhance membrane permeability in drug delivery systems [40-42], and can allow for the delivery of large molecules such as peptides, proteins, nucleotides [43, 44], and therapeutic agents such as insulin and chemotherapeutics, which often face difficulties in being released from biomaterials [27, 45]. Electrical stimulation of membranes loaded with these therapeutics could enhance effective drug delivery by enhancing membrane permeability [46]. The application of electroporation (electropermeability) has been used to develop a new cancer treatment modality, known as electrochemotherapy, over the past two decades [45]. Moreover, drug delivery systems have been employed for the delivery of therapeutics for treatment of diseases such as Alzheimer's disease [39] and epilepsy [47], which require the delivery of agents able to overcome the low permeability of blood-brain barrier.

Locally delivering therapeutics to tissues has been a challenge for a long time. One of the methods used to address this is the design of drug-loaded microchips and their implantation in the vicinity of the tissue needing access to the therapeutics. Microchips provide excellent local and on-demand release of single or multiple drugs and they can deliver a multitude of therapeutic forms, including liquids, solids, and gels [48-50]. The downside of using microchips is that they require invasive surgery for implantation, and implanting microchips for a prolonged time could cause inflammation at the implantation site. Moreover, microchips have a low-capacity reservoir $(<1 \mu \mathrm{L})$ [51]. When higher dosage of drug needs to be delivered, it requires a microchip with a larger reservoir, which can be expensive [26]. To overcome these 
issues, conductive hydrogels $[34,52]$, conductive polymers $[39,53,54]$, and conductive sol-gels [14] have been developed as electrically-responsive drug delivery depots.

For drug delivery with electrical stimulation, direct current (DC) stimulation is commonly used to apply an electric field [55]; however, DC stimulation can be a potential physical and electrical hazard to the skin and tissue at the site of the electrode-tissue interface. Temperature, acid/base, or other types of chemical burns may cause skin irritation, burns, and in extreme cases when tissue is exposed to an electric field for a prolonged period of time, causes tissue necrosis [56]. As a result, local temperature, $\mathrm{pH}$, and electrode overpotential needs to be monitored to avoid tissue irritation and local burns. In addition, using a safe electric current density and duration and conductive paste can minimize the side effects of electrical stimulation $[37,55,56]$.

While exposing tissue to electric fields for prolonged periods can increase the risk of burn and tissue necrosis due to local changes in temperature and $\mathrm{pH}$ [55], controlled local increases of temperature via electrical stimulation, known as radiofrequency ablation, has been used [57]. In this method, electrodes are inserted inside tissue in order to induce controlled tissue necrosis for the treatment of a variety of diseases, including cancer [57, 58]. Moreover, radiofrequency ablation can be combined with chemotherapy, allowing for chemotherapeutic agents to be released in the vicinity of the heated area to enhance the effect of the chemotherapeutic agent [5759]. 


\subsection{Release mechanisms from electrical stimulation}

\subsubsection{Drug release by pressure}

When a DC electric field has been applied to hydrogels between electrodes, it induces anisotropic deformation and ion shift in the hydrogels [60], which causes mobile ions to move toward the counter-electrode, causing local $\mathrm{pH}$ and osmotic pressure changes. As a result, the hydrogels shrink when pressure is increased and swell when pressure is decreased [60-64], and consequently, the pressure on the anode side is not the same as the pressure on the cathode side [65-68]. Additionally, free ions in the surrounding solution move toward the counter-electrode and move into the hydrogel network [69, 70] (Figure 2.1). A uniaxial stress gradient, caused by electrostatic forces, plays an important role in the deformation of hydrogels $[61,65$, 71], and these deformations are reversible so that once an electric field is removed the hydrogel regains its original shape $[29,69,72]$. A change in $\mathrm{pH}$ within a hydrogel can result in a volume change (swelling [37, 47], shrinkage/deswelling [29, 56, 66, 73]), or deformation (bending) $[42,69,74,75]$ ) depending on the hydrogel charge and surrounding electric field strength. As a result, a $\mathrm{pH}$ change in the hydrogel at the site of a working electrode could result in drug release [31].

Both $\mathrm{pH}$ changes and deformation can contribute to the release of drugs from hydrogels. For neutral drugs, one primary mechanism is involved in drug release - the deformation of the hydrogel matrix at the working electrode [66]. However, for charged drugs, three mechanisms are involved in drug release: ion exchange, swelling/deswelling/bending (deformation), and electro-osmosis from anode to 
cathode or vice versa depending on the electric field direction [67]. Figure 2.2 demonstrates the effect of electric field application on drug release. When the electric field is off, there is no matrix deformation, volume change, or drug release (Figure 2.2A). As soon as an electric field is applied the matrix shrinks (Figure 2.2B) or swells (Figure 2.2C) depending on the hydrogel properties, allowing for effective release of the imbibed drugs.

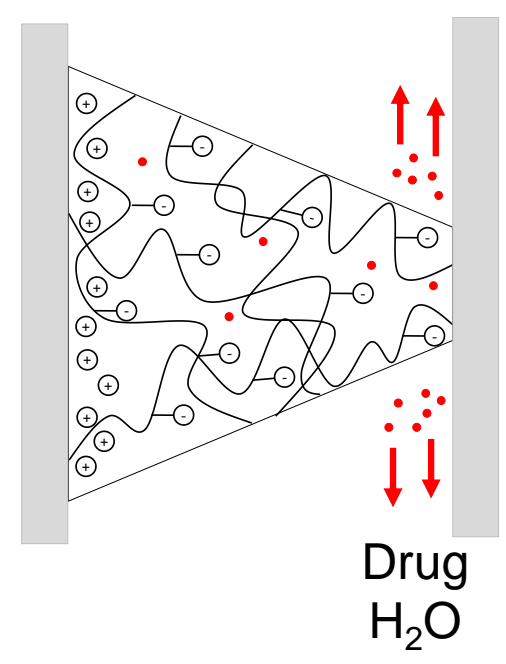

Figure 2.1. Hydrogel deformation that occurs when an electric field has been applied. 
A Electric field

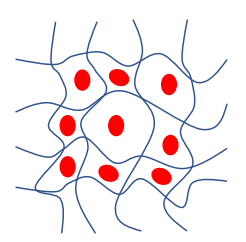

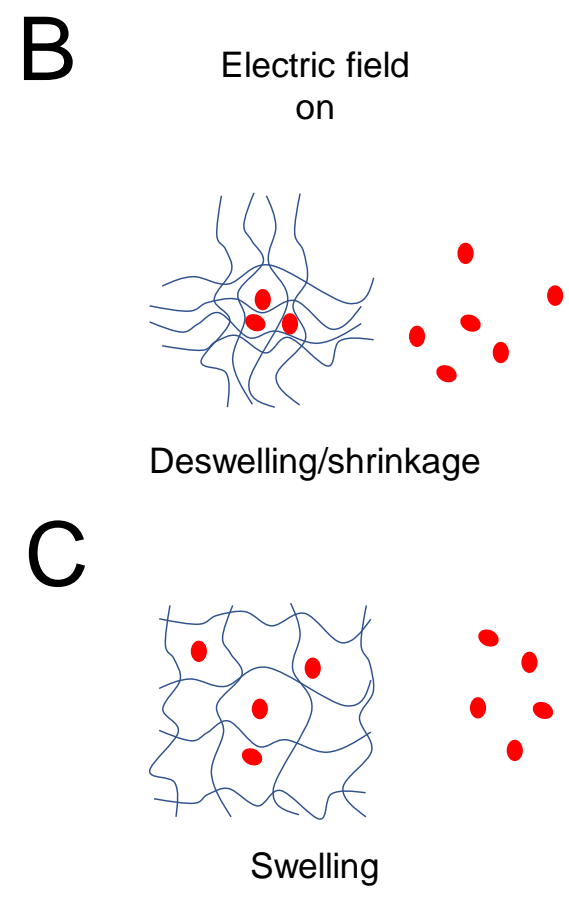

Swelling

Figure 2. Matrices deform and drug releases once an electric field is applied. Representative hydrogel microstructure with (A) no electric field, (B) electric field applied with matrix shrinkage, (C) electric field applied with matrix swelling.

\subsubsection{Drug release by matrix degradation}

Electrical stimulation of hydrogels can result in the migration of ions towards the counter-electrode, which can result in localized changes in $\mathrm{pH}$. As a result, a change in $\mathrm{pH}$ can cause matrix degradation and drug release [76-80]. Moreover, the rate of drug release depends on $\mathrm{pH}$, where the drug release rate is higher at lower $\mathrm{pH}$ and decreases when the $\mathrm{pH}$ increases [76]. Figure 2.3 illustrates the degradation of a hydrogel matrix when an electric field is applied, and Figure 2.4 demonstrates how polymer weight loss can occur when an electric field is applied. 
A

B

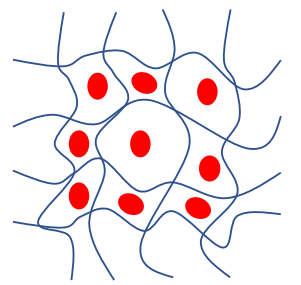

Electric field

off

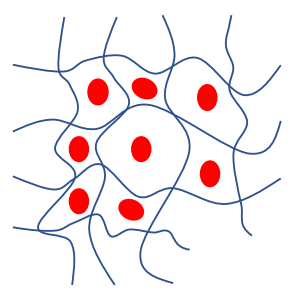

Electric field

on

Figure 2.3. Schematic of hydrogel degradation and drug release upon applying an electric field. (A) no electric field is applied and no drug release occurs, and (B) degradation and drug release occurs as a result of an applied electric field.

A

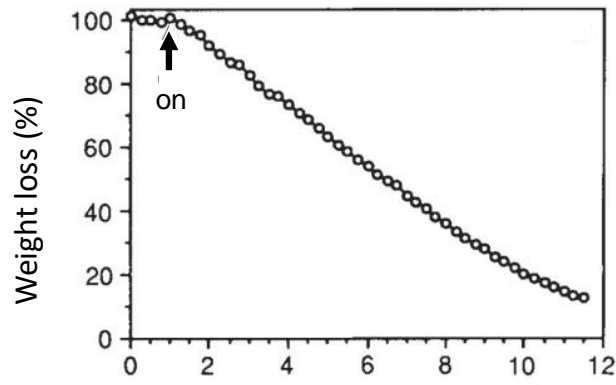

Time (h)
B

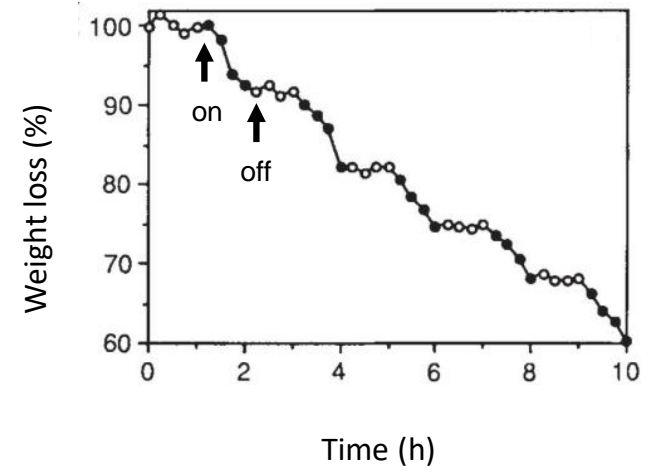

Figure 2.4 Surface erosion of a matrix can occur upon applying an electric field. Graphs show weight loss of poly(ethyloxazolion)-poly-(methacrylic acid) complexed in $0.9 \%$ saline when $10 \mathrm{~mA}$ current is passed with a (A) continuous current or (B) step-function electric current [70]. 


\subsection{Parameters affecting drug release via electricity}

For electrically-controlled drug release from biomaterials, sol-gels [81, 82], hydrogels [66, 76], and conductive polymers [35, 83] have been used as drug reservoirs. For typical drug delivery systems, hydrogels are more common than conductive polymers, since conductive polymers have certain limitations and are only needed in certain applications. For example, only charged drugs with certain sizes can be released from biomaterials using electrical stimulation [52, 84]. Hydrogels offer many advantages in drug delivery applications including their tunable mechanical properties, biocompatibility, biodegradability, and high water content $[28,62,68]$. Additionally, the three-dimensional structure of hydrogels allows for the immobilization of large molecules such as protein, peptides, and DNA-based drugs $[85,86]$. Unfortunately, hydrogels on their own typically exhibit poor electrical behavior [87]. One type of hydrogel that can be used to overcome this limitation is electrolyte hydrogels, which can be used for controlled released [37, 64, 66]. In addition, materials can be incorporated into hydrogel to improve their conductivity. These materials include conductive polymers [30, 35], conductive polymer nanoparticles [78, 81], carbon nanotubes [56, 88], dielectric nanoparticles [30], graphene [89] and metal nanoparticles such as silver [90]. In addition to enhancing conductivity these materials can also reduce diffusion of drug from hydrogel [37], and thus this limitation needs to be taken into consideration when designing electricallyconductive hydrogel systems for drug delivery applications.

\subsubsection{Effect of drug charge and size}


Adequate characterization of the molecular interaction of matrices with drugs is critical for developing an effective and reliable electrically-stimulation drug delivery system. One of the most import factors that needs to be taken into consideration in the design of matrix formulations is drug charge [32, 66, 70]. Although the delivery of ionic drugs is common with electrical stimulation, non-ionic drugs also can be loaded to matrixes for this application, but often at lower concentrations than ionic drugs. The molecular interactions between charged drug and charged matrix can increase the loading capacity of hydrogels [70]. In addition, drug release rates decrease with increasing drug size [32] and Figure 2.5 demonstrates that larger drug sizes results in lower drug release rates.

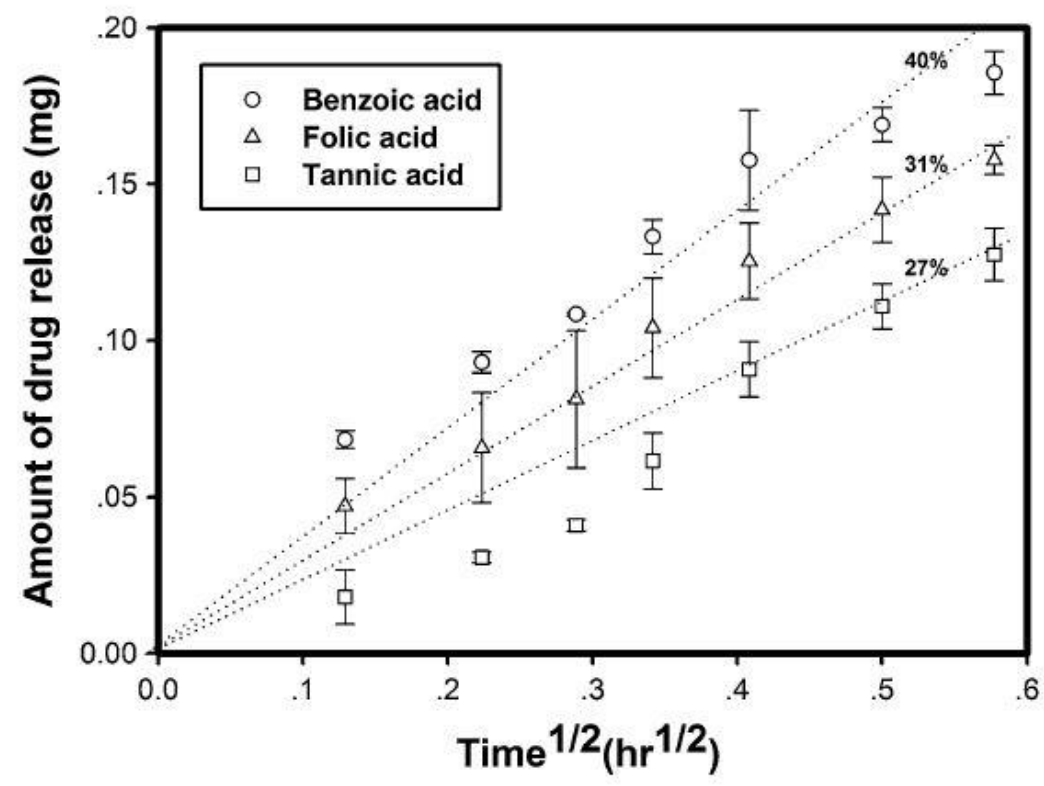

Figure 2.5 Drug release rate decreases with increasing drug size. Tannic acid has the largest size and therefore has the lowest release rate. Benzoic acid has the smallest size and hence has the highest release rate. Benzoic acid $\mathrm{M}_{\mathrm{w}}=122$. Folic acid $\mathrm{M}_{\mathrm{w}}=$ 441, tannic acid $\mathrm{M}_{\mathrm{w}}=1701$ [32]. 


\subsubsection{Effect of matrix concentration and cross-linking}

Increasing matrix concentration and charge will enhance its response to electric fields [64, 91], thereby impacting the release of drugs from the systems. Another factor that can affect the release rates of drugs from matrixes is the crosslinking ratio of the hydrogel systems. Increasing the cross-linking ratio decreases the porosity of hydrogel resulting in a decrease in the drug release rate [34, 92-05]], as shown in Figure 2.6. Increasing the cross-linking ratio also decreases molecular mobility with in the hydrogel matrix [96]. Polymer molecular weight affects the crosslinking ratio of hydrogel systems, where shorter molecular weight chains result in larger free volumes and chain ends within the hydrogels [75]. In addition, increasing cross-linking density improves the mechanical properties of hydrogels [96].

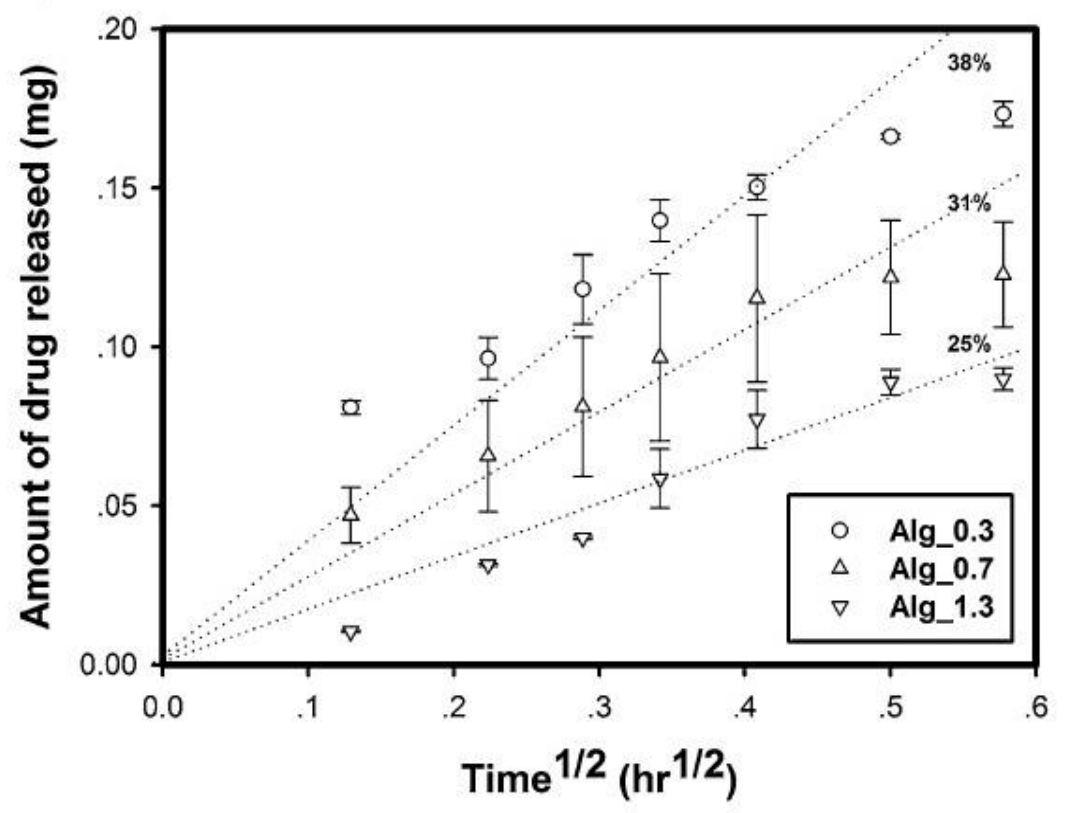


Figure 2.6. Increasing the cross-linking ratio in hydrogels decreases the rate of drug release. Amount of folic acid released from alginate-calcium (Alg-Ca) hydrogels with $0.3,0.7$, and 1.3 cross-linking ratios [32].

\subsubsection{Effect of adding conductive materials}

Hydrogels that are responsive to electrical stimulation are sometimes known as electrolyte hydrogels [64]. In general, the addition of conductive agents to electrolyte hydrogels will enhance their conductivity $[34,56,62,80,82,91]$ and after electrical stimulation, increase drug release [34, 62]. Samples with higher conductivity can deform more significantly, which can result in enhanced drug release [56, 73, 98]. Despite these advantages, there is a limit for increasing the amount of conductive additives to hydrogels, as increasing too much can result in sedimentation of the conductive agent $[56,73,99]$, or not significantly improve the conductivity of the hydrogel system [62]. Moreover, high loading of a conductive agent can result in increased cross-linking between the polymer chain added agent, thereby restraining chain movement so that the hydrogel exhibits a smaller mesh size, less swelling, and hence a minimized drug release rate $[34,68,100]$. If a large amount of conductive nanoparticles are loaded into a hydrogel, they have the potential to allow for a burst of drug out of the hydrogel and/or result in local damage to the matrix due to stress of removing the electric field (due to matrix deformation while electric field is applied). For multi-pulsatile release profiles, it is imperative to choose a conductive agentpolymer ratio which prevents diffusion [62] and allows for the constant release of drug during stimulation without damaging the matrix [56]. Figure 2.7 demonstrates the 
effect of adding conductive agent to hydrogel on drug release rates and Figure 2.8 shows the effect of adding graphene and carbon nanotubes to hydrogels on mechanical and electrical properties.
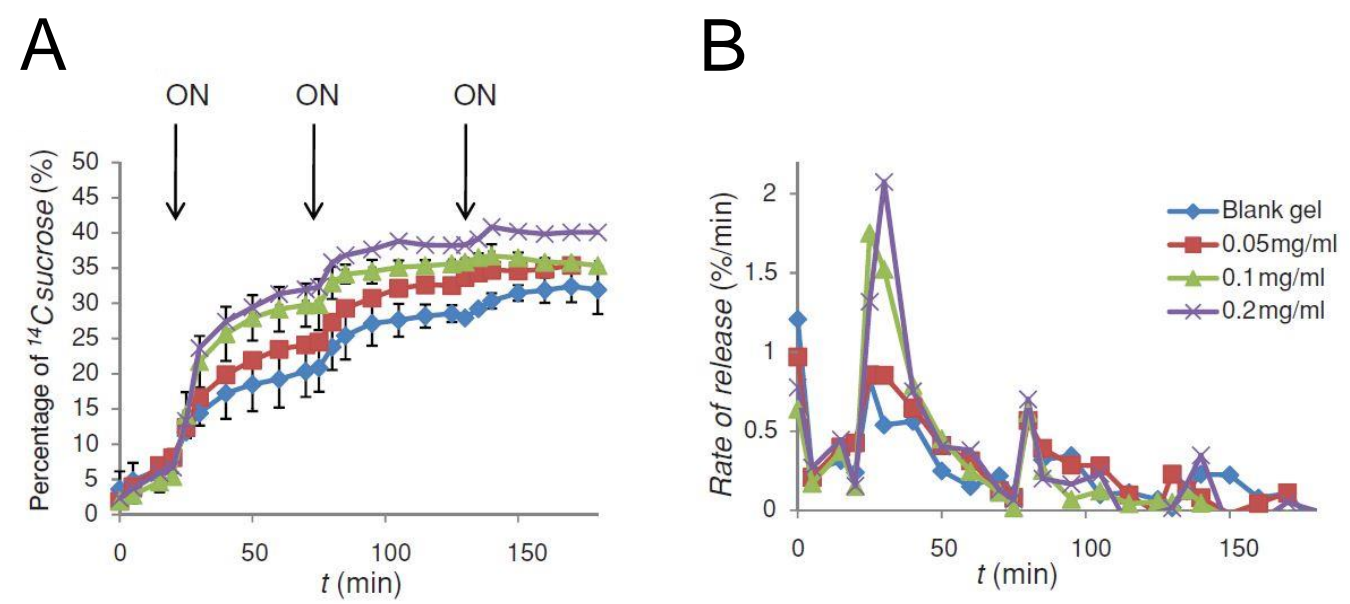

Figure 2.7. Increasing the amount of conductive agent enhances the drug release

rate. (A) Pulsatile drug release from a hydrogel upon applying electric field. Drug release was monitored while applying an electric field for 5 minutes every 60 minutes. The pulsatile release of C-sucrose was determined for blank hydrogels and hydrogels with $0.05 \mathrm{mg} / \mathrm{ml}, 0.1 \mathrm{mg} / \mathrm{mls}$ and $0.2 \mathrm{mg} / \mathrm{ml}$ of pMWNT (pristine multi-wall carbon nanotubes). (B) C-sucrose release rates from the described hydrogels. Rates of release were calculated over time upon exposure to $10 \mathrm{~V}$ DC electric field [56]. 

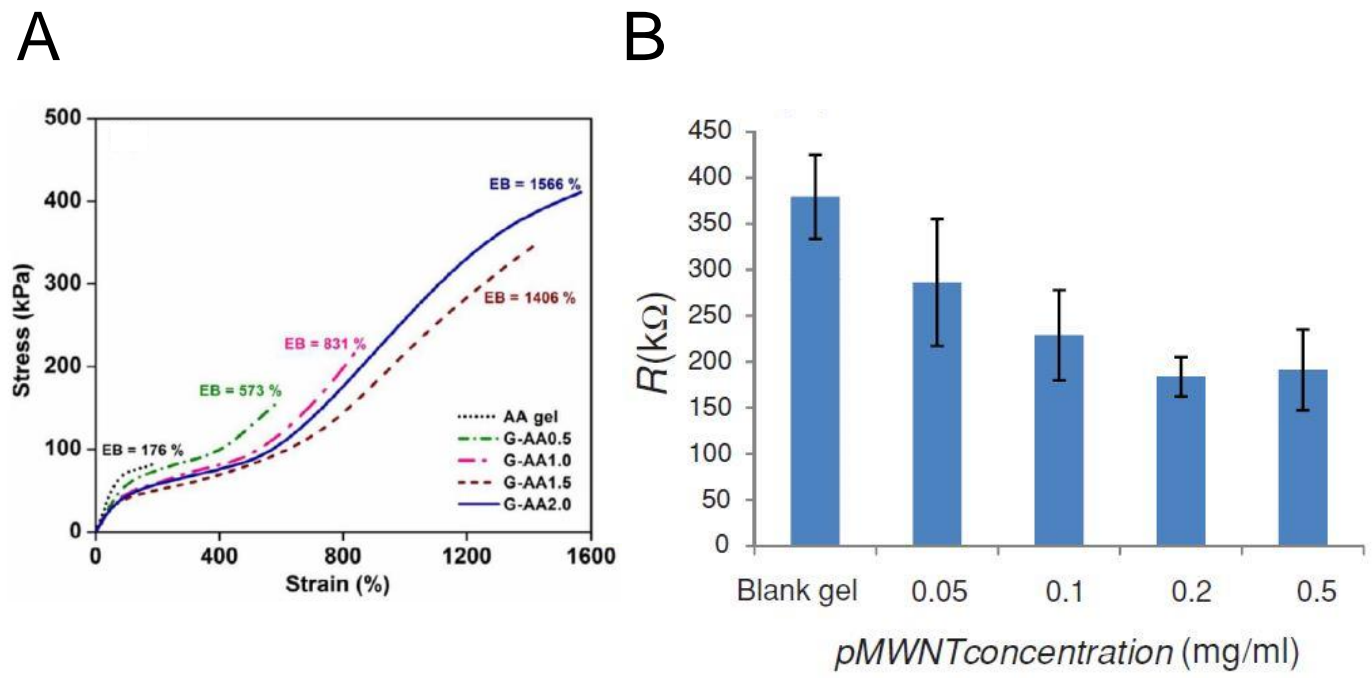

Figure 2.8. Adding conductive agents to hydrogels enhance their mechanical properties and improves their electrical properties. (A) Stress-strain curve for an acrylic acid hydrogel with and without graphene. Increasing the amount of graphene particles in the hydrogel increases hydrogel stiffness and toughness [65]. (B) Electrical properties of poly(methacrylic) acid hydrogel improves with the addition of carbon nanotubes, where increasing carbon nanotube concentration decreases resistance [59].

\subsubsection{Effect of electric field polarity}

Electric field polarity is one the most important factors in the controlled release of drugs from electrically-responsive materials. Electric fields generate a driving force that enhances drug release by generating electro-repulsive forces between the drug and electrode with the same charge, which increases the rate of drug release $[28,32,34$, 84, 88]. Pairatwachapun et al. showed that the release rate of a positively charged drug is the highest when a cathode (negatively charged electrode) is placed on the hydrogel and in comparison, to an anode (positively charged electrode). Similarly, Niamlang et 
al. [84] demonstrated that the drug release rate of a negatively charged drug is highest under a cathode. Figure 2.9 shows the release of a negatively charged drug under a cathode and anode. The negatively charged electrode generated electro-repulsion forces towards the negatively charge drug, and consequently had a higher release rate.

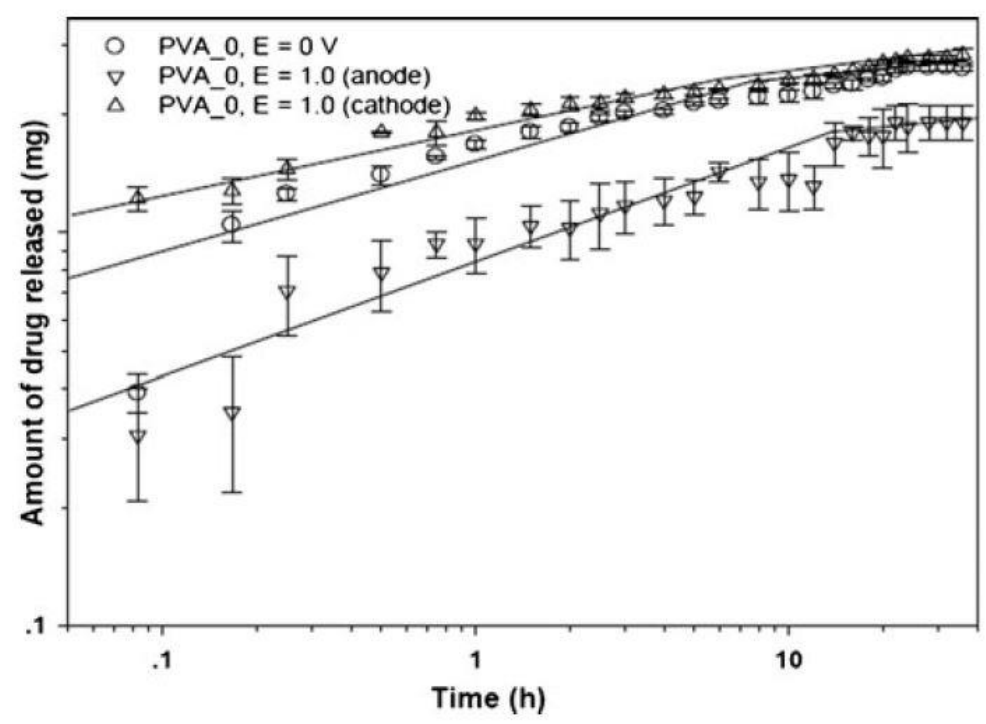

Figure 2.9. Drug release in response to electrical stimulation of opposite polarity.

Release of benzoic acid from a benzoic acid-loaded poly(vinyl acid) hydrogel in relation to time with samples exposed to a cathode or anode [33]. 


\subsubsection{Effect of electric field strength}

Electric field strength has a significant impact on drug release from electrically-responsive hydrogels, where increasing voltage can result in an increase in the drug release rate $[32,33,51,56,92,94,99,102]$. The deformation of conductive hydrogel matrices depends on the electric field intensity and hydrogel charge. With increasing voltage, deformation increases [42, 69, 74]. Increasing electric field strength can increase the shrinkage at the anode site and swelling at the cathode site, which both result in increased drug release rate $[33,67,94]$. Figure 2.10 demonstrates the effect of increasing electric field strength on drug release rate, where increasing voltage resulted in an increase in the drug release rate.

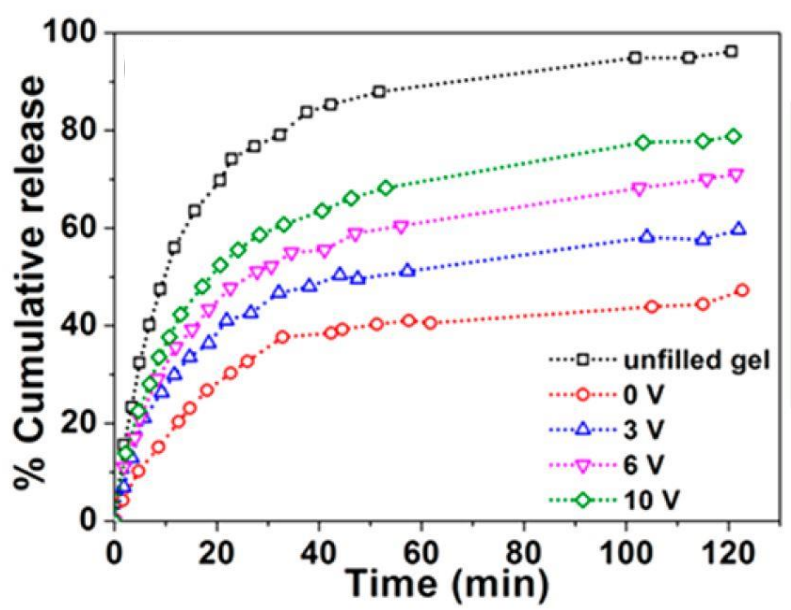

Figure 2.10. Drug release rate increases with increasing electric field strength. Drug release from poly(AA-co-AMPS)/carrageenan/RGO (poly(acrylic acid-co-2acrylamido-2-methyl-1-propanesulfonic acid)/carrageenan/reduced graphene oxide) hydrogels with different electric field strengths. Samples was placed between parallel electrodes $500 \mathrm{~mm}$ apart $[62,68]$. 


\subsubsection{Effect of duration of an applied electric field}

Increasing the exposure time of matrixes to electric fields can enhance drug release $[29,35,92]$. At the same time, increasing the duration of an applied electric field can result in an increase in temperature, especially for a hydrogel network with high impedance [56]. Any increase in temperature above $41-42{ }^{\circ} \mathrm{C}$ during electrical stimulation can induce tissue necrosis and should be avoided [89]. Seravant et al. showed that by adjusting strength and duration of an applied electric field, the temperature increase could be controlled to minimize its effect on the environment surrounding the implanted hydrogel. Fortunately, drug release from electricallystimulated hydrogel matrices can be fast. For example, drug release can be successfully achieved upon applying an electric field of $1 \mathrm{~V}$ for 10 seconds [78]. Carbon nanotubes exhibit excellent thermal conductivity and exposing a carbon nanotube-loaded hydrogel to a DC electric field (10 V for 2 minutes) resulted in an increase in temperature up to $15{ }^{\circ} \mathrm{C}$ in the bulk hydrogel [89].

\subsubsection{Effect of pH}

Solution $\mathrm{pH}$ affects the release rate of drugs from hydrogels, where negatively charged drugs exhibit a higher release rate at higher $\mathrm{pH}(\mathrm{pH}=8)$ than neutral or positive states $(\mathrm{pH}=5)$ due to electro-repulsion forces [88]. For systems that are $\mathrm{pH}$ sensitive, drug release solely depends on an applied electric field at neutral $\mathrm{pH}$ [99]. As mentioned earlier, electrical stimulation can result in $\mathrm{pH}$ changes in a hydrogel system, where the $\mathrm{pH}$ change occurs locally around electrode and not in the bulk of the hydrogel. $\mathrm{pH}$ changes in hydrogel systems should be monitored, since an increase 
in $\mathrm{pH}$ above 13 can result in tissue necrosis [37]. To avoid tissue damage, electric field strength and application time should be monitored closely, where with increasing applied voltage, exposure time should be decreased [37].

\subsubsection{Effect of direction of electric field}

Strain in hydrogels following deformation is due to the pressure and therefore this effect is isotropic. As a result, drug release from hydrogel does not depend on the direction of an applied electric field and it can be applied from any direction [106].

\subsubsection{Effect of porosity and mesh size}

Larger pore sizes in hydrogels enhance drug and ion mobility in and throughout the hydrogel matrix $[64,79,107]$, and as a result the quantity of drug released is higher from hydrogels with larger pore sizes. Larger pore sizes can be obtained within hydrogels by using a smaller crosslinking ratio [33, 34, 84, 92]. Figure 2.11 demonstrates the correlation of drug diffusion from a hydrogel with the hydrogel mesh size and cross-linking ratio, where drug diffusion is enhanced by increasing the mesh size and diffusion decreases by increasing the cross-linking ratio. 


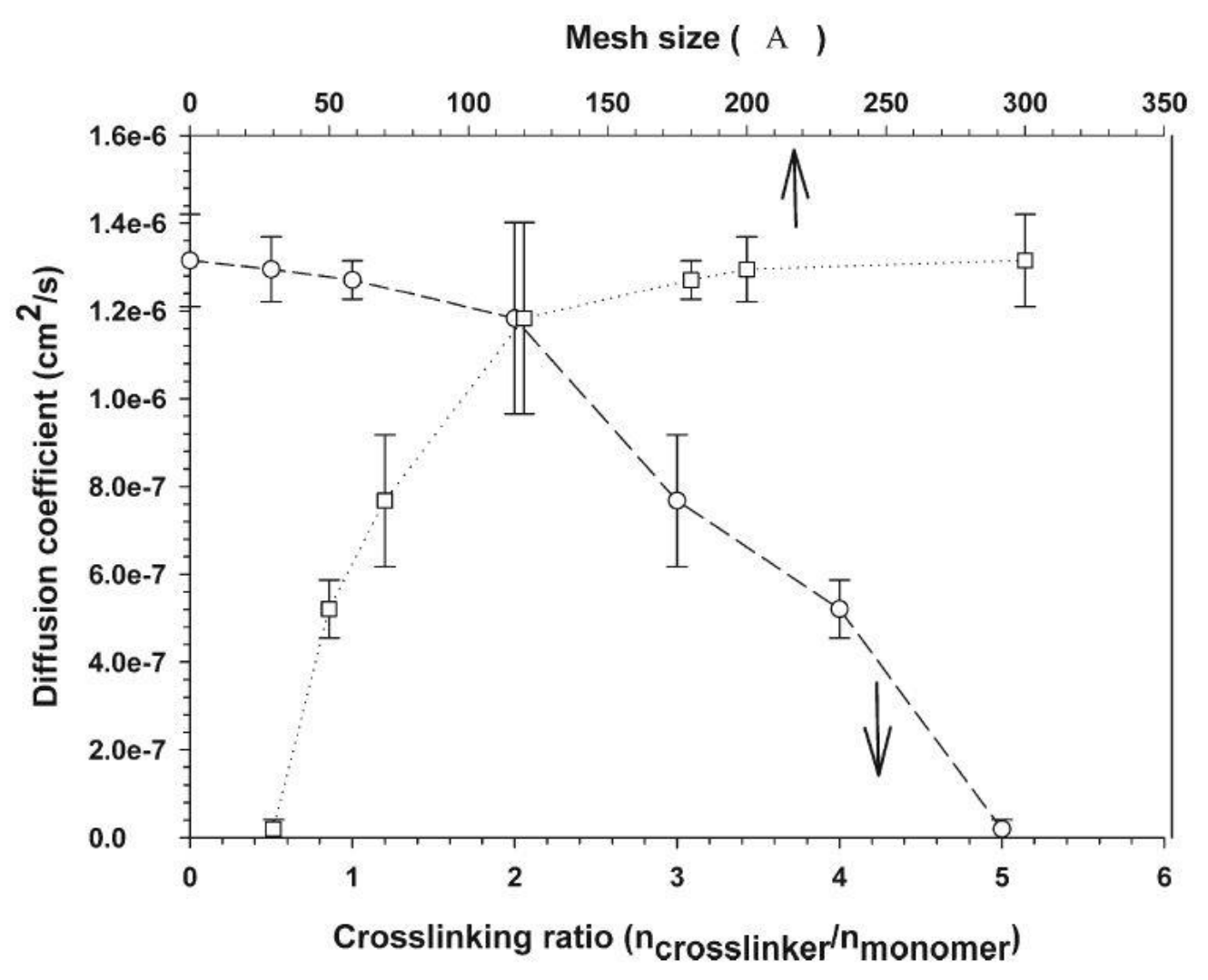

Figure 2.11. Diffusion increases with increasing mesh size and decreases with increasing cross-linking. Diffusion coefficient of benzoic acid from poly(vinyl alcohol) hydrogels in relation to mesh size and cross-linking ratio without presence of electric field [33].

\subsubsection{Effect of diffusion}

Depending on drug and hydrogel/polymer interactions, passive diffusion of the drug from the hydrogel matrix can occur $[62,84]$. In order to eliminate passive diffusion of drugs from hydrogel networks and to improve on-demand drug delivery, dopants have been added to a wide variety of hydrogel systems. Conductive polymer nanoparticles $[31,78,81]$ and carbon nanotubes have been used to prevent passive 
drug release and improve on-demand drug release from drug depots [31, 78, 81]. Figure 2.12. demonstrates that by encapsulating a drug into conductive nanoparticles, passive diffusion (cumulative release) of the drug from matrix can be prevented. Drug release rate from dopants depends on the molecule size and exposure time [31]. Increasing dopant concentration will increase release rate up to a point, and increasing it beyond a certain concentration will no longer enhance release [61].

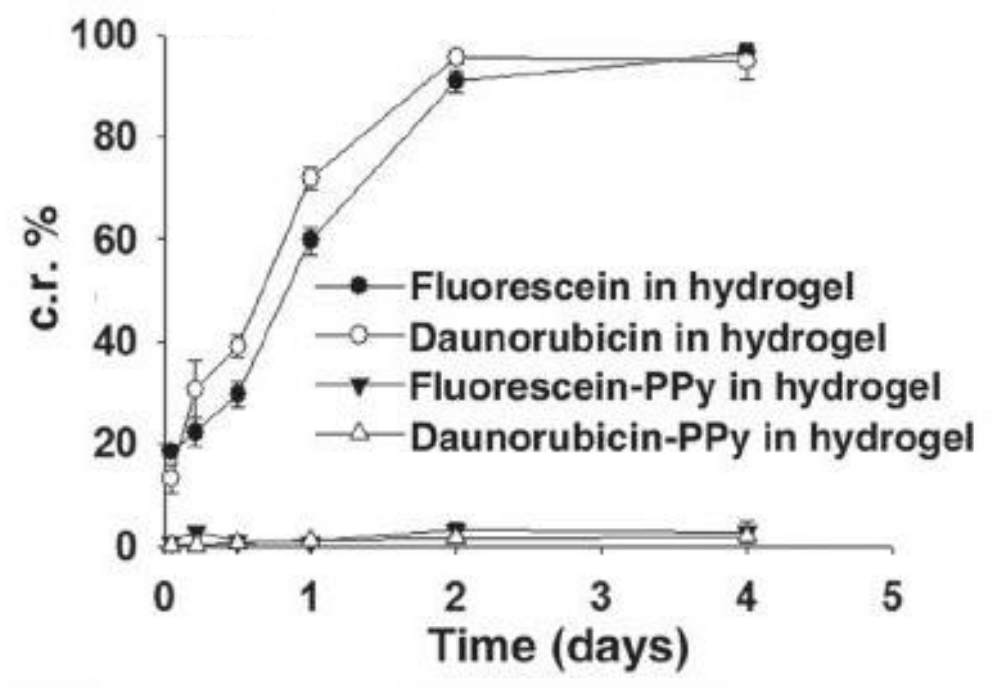

Figure 2.12. Cumulative release (c.r.) from hydrogel can be prevented by encapsulating drug with nanoparticles. Cumulative release is observed from hydrogel with nanoparticles of polypyrrole [81].

\subsubsection{Effect of applying electric field on tissue permeability}

Electrical stimulation of tissue can increase the permeability of skin. Figure 2.13 shows a schematic of how cell permeability is altered by applying an electric pulse $[40,41,94,108]$. When an electric pulse is applied to the skin pores are 
generated in the cellular membrane, allowing for the penetration of drugs through the membrane [46], which provides a unique opportunity to introduce large therapeutic molecules into tissue [40, 109]. Skin acts like an ion exchange membrane when it comes to the diffusion of drugs through the skin, where negatively charged molecules diffuse more slowly than positively or neutrally charged molecules. Overall, skin permeability will increase with increasing surface negative charge [34, 108]. Figure 2.14 demonstrates the effect of increasing electric field strength on drug permeation, whereas the electric field strength increases, drug permeation increases. Additionally, increasing the amount of conductive agent present in a drug delivery vehicle can increase skin permeability [108]. Figure 2.15 shows the effect of increasing the amount of conductive agent in a hydrogel system on the penetration of a drug through the skin, where by increasing the charge of a lipogel, skin penetration increases.

Electrotherapy also can be used to neutralize or destroy the active sites of growth factor enzymes. This application is important in that the concentration of growth factors is exponentially higher in cancerous cells in comparison to healthy tissue. In addition, low density DC electrotherapy can halt cell proliferation [110]. For example, electrical stimulation of skin can destruct the stratum corneum, and the cell structure becomes loose, thereby increasing cell infiltration in the dermis and degenerating skin appendages, which results in an increase in skin permeation [94]. 
A

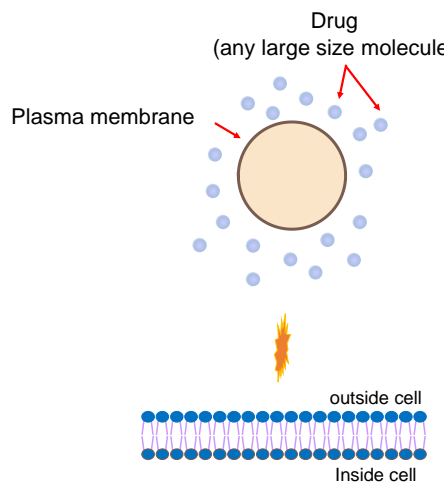

Introducing

drug to the cell
$\mathrm{B}$
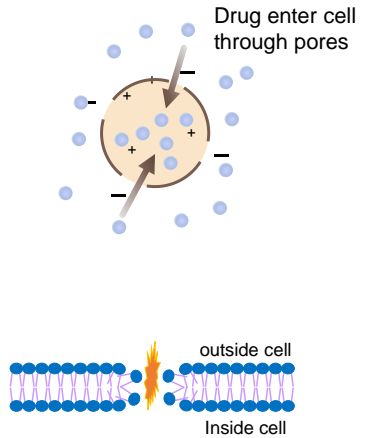

Applying electric pulse; pores generated in the plasma membrane and drug
C
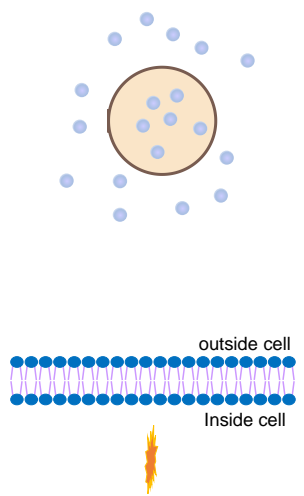

after electric pulse; pores disappear and drug remains inside cell.

Figure 2.13. Electrical stimulation increases porosity of the cellular membrane by generating pores. (A) Drug cannot diffuse into cell due to drug size (no electrical stimulation). (B) Pores form in cell membrane and drug enters following electric stimulation. (C) Membrane reforms once electrical pulse is removed and drug can no longer penetrate.

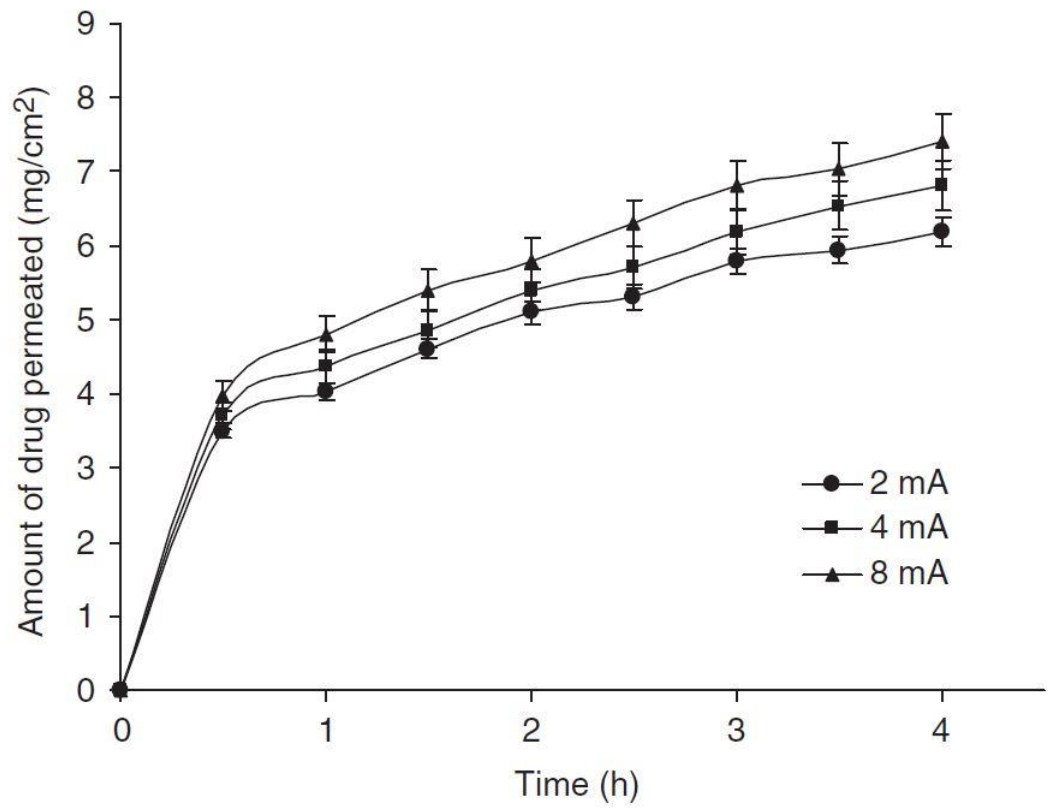


Figure 2.14. Increasing electric field strength can enhance drug permeation through skin. Effect of current strength on permeation of ketoprofen through skin [94].

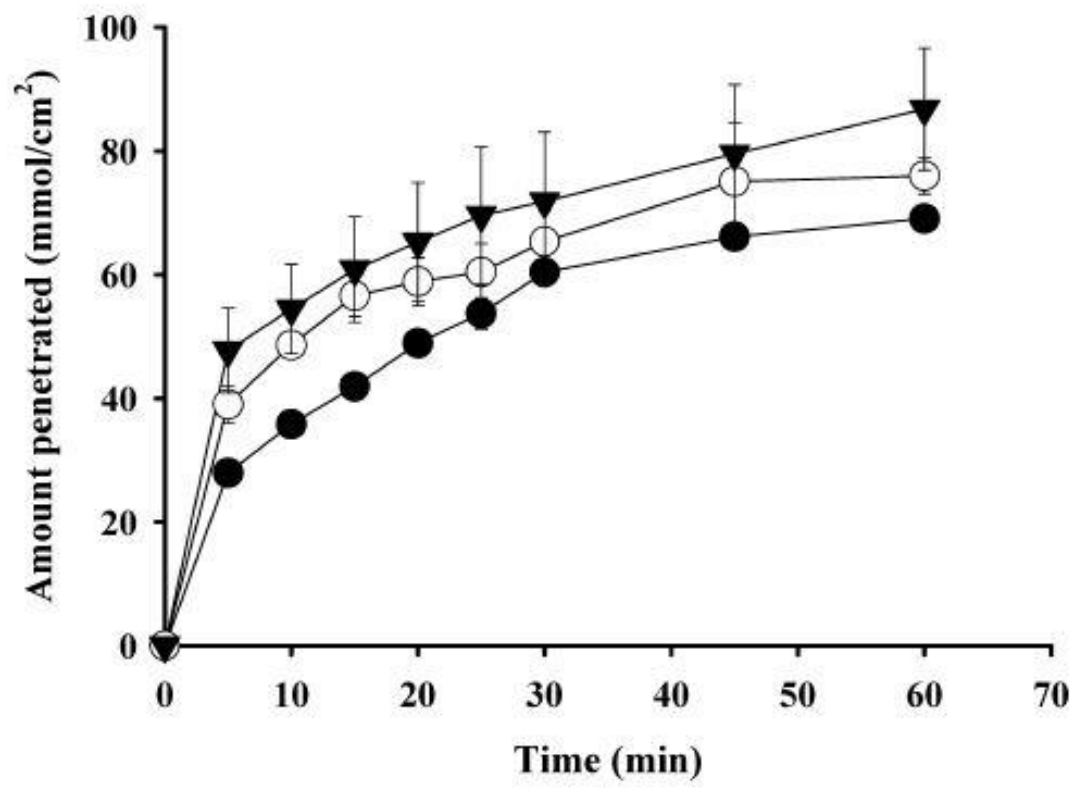

Figure 2.15. With increasing surface charge skin permeation increases. In vitro skin permeation profiles showing ascorbyl palmitate by cathodal transport. $\bullet$ neutral hydrogel, $\bigcirc \quad 10 \%$ DCP (dicetylphosphate) negative lipogel, $\quad \nabla 20 \%$ DCP (dicetylphosphate) negative lipogel [108].

\subsubsection{Biocompatibility}

Biocompatibility is an essential requirement for biomaterials used for controlled drug delivery applications [86]. High cell viability and proliferation can show that biomaterials are biocompatible $[28,62,68,111]$. Figure 2.16 shows how 
hydrogels have the potential to be biocompatible since cell viability and proliferation on the hydrogels is high and that all samples maintained their normal cellular metabolism. Histology testing on tissue surrounding hydrogels with and without applying an electric field has demonstrated that hydrogels can be biocompatible [56, 68, 80]. For example, Figure 2.17 shows the potential for hydrogels to be biocompatible upon implantation. Since $\mathrm{pH}$ changes were only observed in the vicinity of electrodes and not in the surrounding tissue, these changes do not significantly affect cell growth [37]. Additionally, hydrogels can provide a substrate for cell adhesion and growth [82, 103, 104] making them physiologically reliable biomaterials for long-term pulsatile drug release [21].

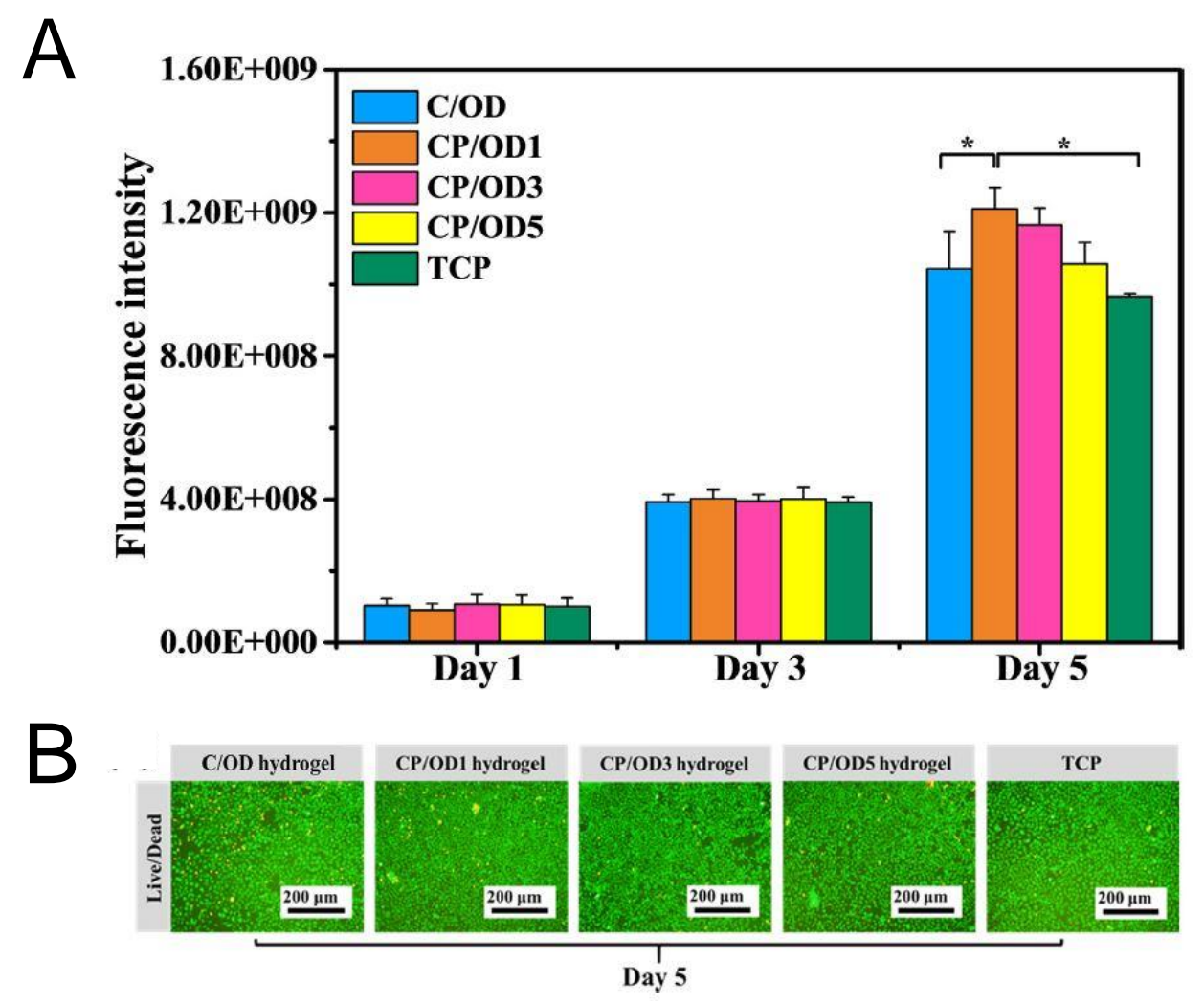


Figure 2.16. Conductive hydrogels are biocompatible, and cells maintain normal cell metabolism in direct contact with conductive hydrogels. (A) Cell proliferation of L929 cells exposed to conductive hydrogels and TCP (mean for $n=4 \pm$ standard deviation). (B) Live/dead staining of L929 cells at successive culture periods for CP/OD1, CP/OD3, CP/OD5, C/OD and TCP, respectively. C/OD (chitosan/oxidized dextran), CP/OD1 (chitosan-graft-polyaniline/1 wt \% oxidized dextran), CP/OD3 (chitosan-graft-polyaniline/3 wt \% oxidized dextran), CP/OD5 (chitosan-graftpolyaniline/5 wt \% oxidized dextran) and TCP (tissue culture plate) [28].
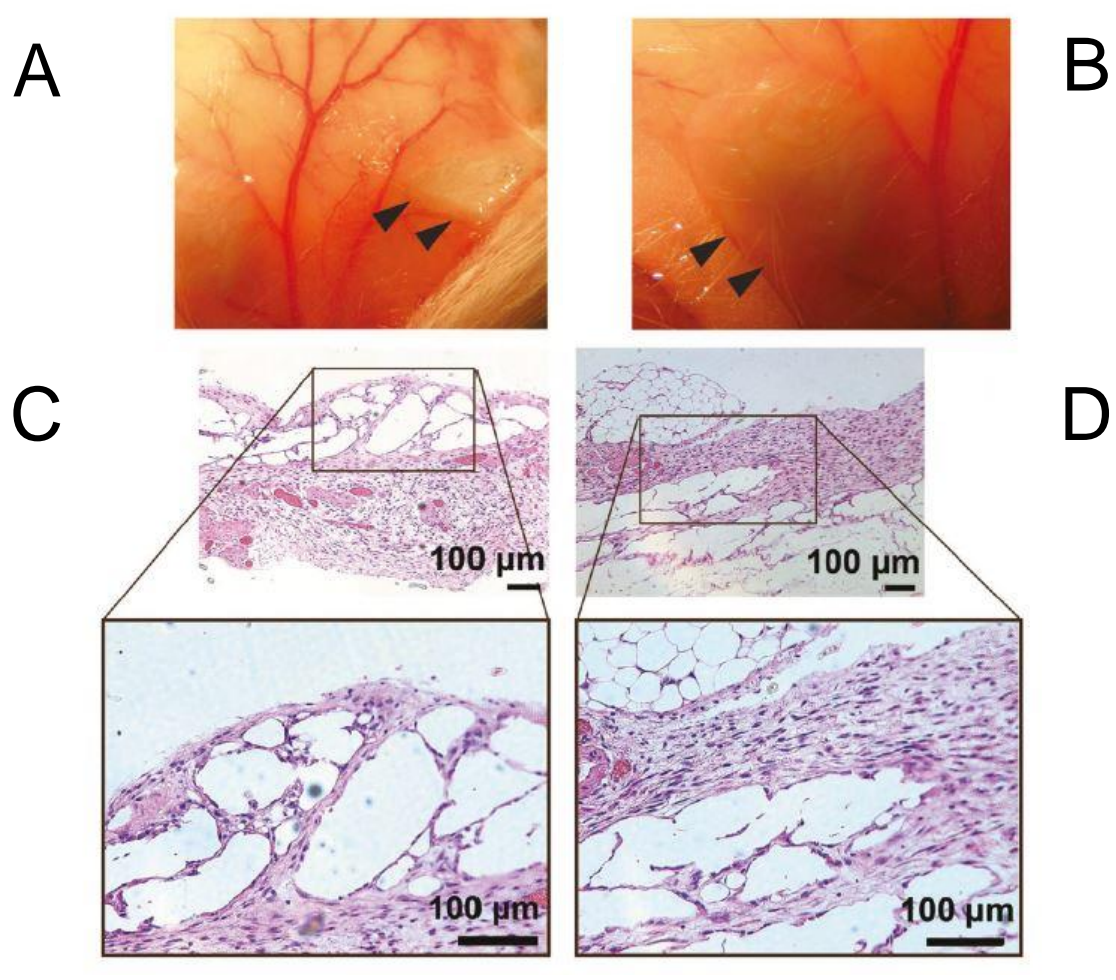

Figure 2.17. Conductive hydrogels are biocompatible. Figure (A) and (B) show the formation of a conductive hydrogel with 1 wt $\%$ polypyrrole nanoparticles after subcutaneous injection in FVB mice. Black arrow indicates implants after (A) one 
week and (B) two weeks post implantation. Figures C and D demonstrate hematoxylin and eosin (H\&E)-stained images of tissues exposed to conductive hydrogels after subcutaneous injection into FVB mice (C) one week and (D) two weeks after implantation. H\&E-stained cells can be seen in the hydrogel areas [81].

\subsubsection{Distance of electrode from depot}

Drug release from drug reservoirs has been studied under a variety of conditions and generally electrodes are either in direct contact with the reservoir or electrodes [73]. In transdermal drug delivery and iontophoresis drug delivery, when an electrode is in direct contact with the skin and electrode, drugs were able to permeate through skin $[34,37,62]$. This method is one of the most common methods of drug delivery with electrical stimulation. Increasing the thickness of the drug reservoir can decrease permeation of the drug, likely due to the length of diffusion path [64, 94]. This issue can be resolved by increasing electric field strength which increases both drug permeation and diffusion rates.

Most of these delivery systems require implantation of drug reservoir prior to stimulation. In subcutaneous drug delivery, the reservoir is implanted under the skin where the electrode and matrix are not in direct contact with one another, and the electrode is in direct contact with skin during stimulation. As discussed earlier, this direct contact might cause inflammation and tissue necrosis at the site of electrode [56]. By carefully selecting the electric field intensity, duration and polarity this issue could easily be overcome. Figure 2.18 shows an example of subcutaneous drug delivery process. 
Drug delivery to deep tissue is always challenging. One of the methods that has been developed to overcome this challenge is the integration of MEMS (MicroElectro-Mechanical Systems) with electro-responsive hydrogels, and fabrication of these implantable delivery devices for prolonged controlled drug release $[29,50]$. In recent years, there has been a significant amount of effort to deliver drugs to areas which are not easily accessible, such as the central nervous systems, which has low access due to limited permeability across the blood brain barrier [39]. The olfactory region is the only site in the human body that has direct contact to the central nervous system. Recently, delivering drug to the brain via the olfactory region has been proposed, where drugs would diffuse through olfactory mucosa and reach the central nervous system [112].

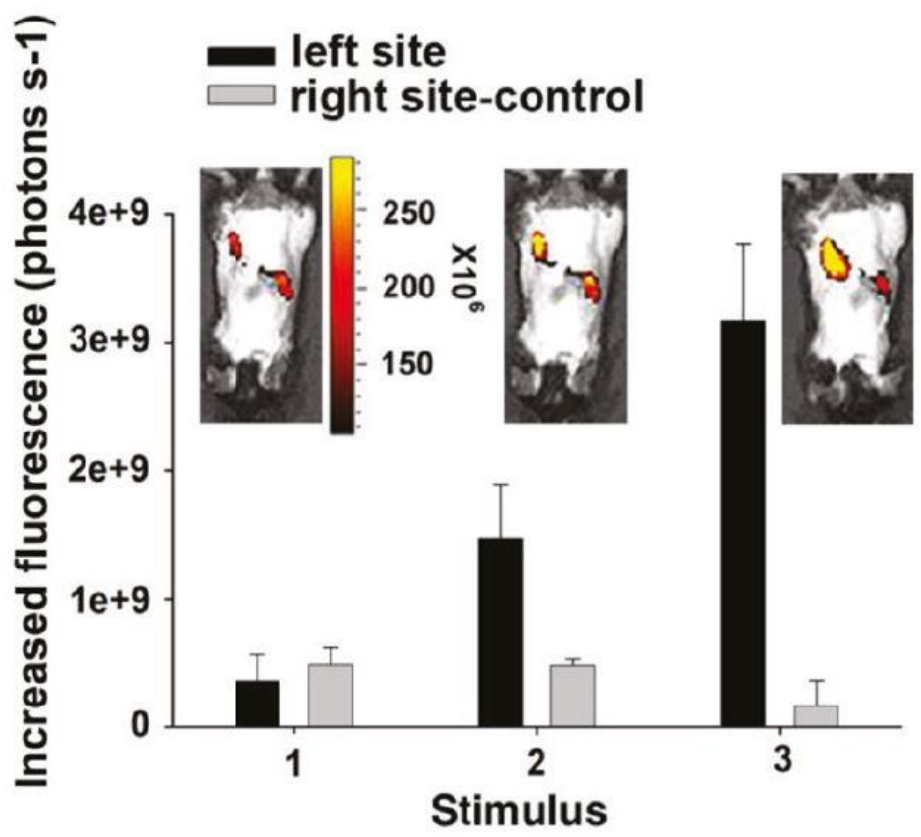


Figure 2.18. In vivo on-demand drug release from conductive hydrogels. In vivo fluorescent images after applying $-1.5 \mathrm{~V} / \mathrm{cm}$ to the implanted conductive hydrogel on the left side. The right side hydrogel is the control as no electric voltage was applied to it. The unit of scale of the right side of the mouse image is photons per steradian per second. (1) Before applying electric field and (2) \& (3) after applying voltage for 40 seconds [81].

\subsection{Current efforts on multi-drug delivery using electrically responsive materials}

Nanoparticles are promising depots for on-demand multi-drug delivery via electrical stimulation. By encapsulating drugs with different charges within nanoparticles on-demand release can be achieved by applying an electric field [81].

Luo et al. [113] developed a system consisting of polypyrrole nanoparticles within polypyrrole film. It incorporated a drug into the polymer as a dopant and integrated another drug into the nanoholes inside the polymer films. Figure 2.19 demonstrates on-demand multi drug-release from conductive hydrogel by controlling electric field polarity. In addition, microchips can be easily programmed for multi-drug release [48], as shown in Figure 2.20.
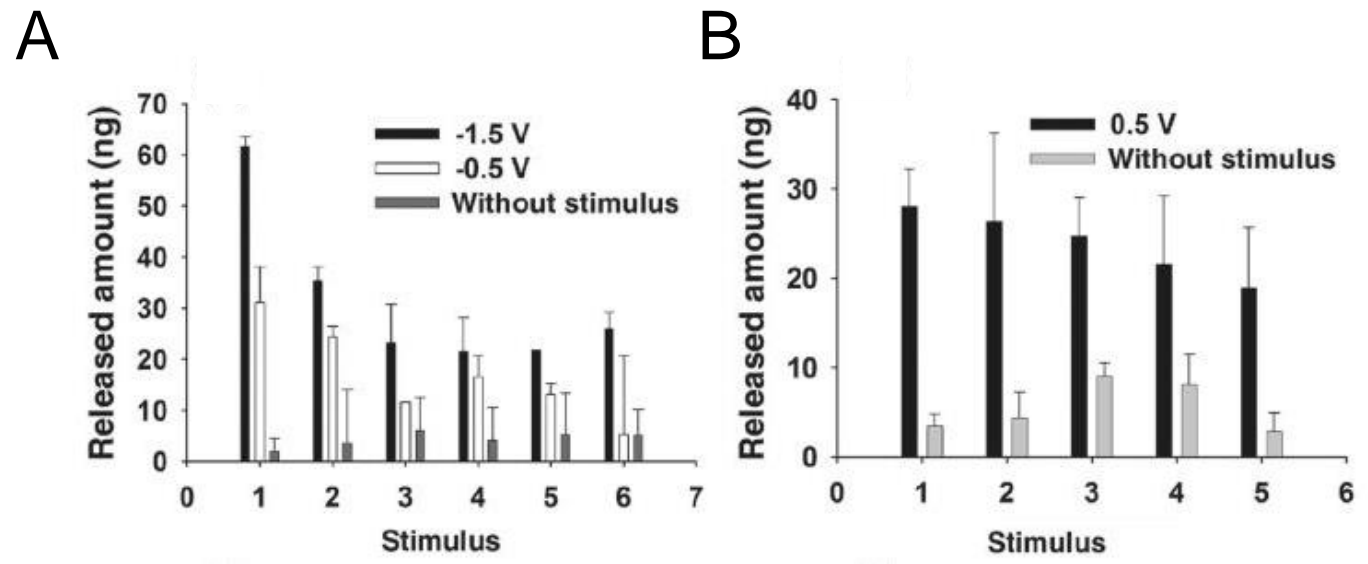
Figure 2.19. On-demand pulsatile multi-drug release from conductive hydrogel. (A) Release of fluorescein in PBS following application of $-0.5 \mathrm{~V}$ and $-1.5 \mathrm{~V}$ for 10 second every 5 minutes. (B) Release of daunorubicin in PBS following application of

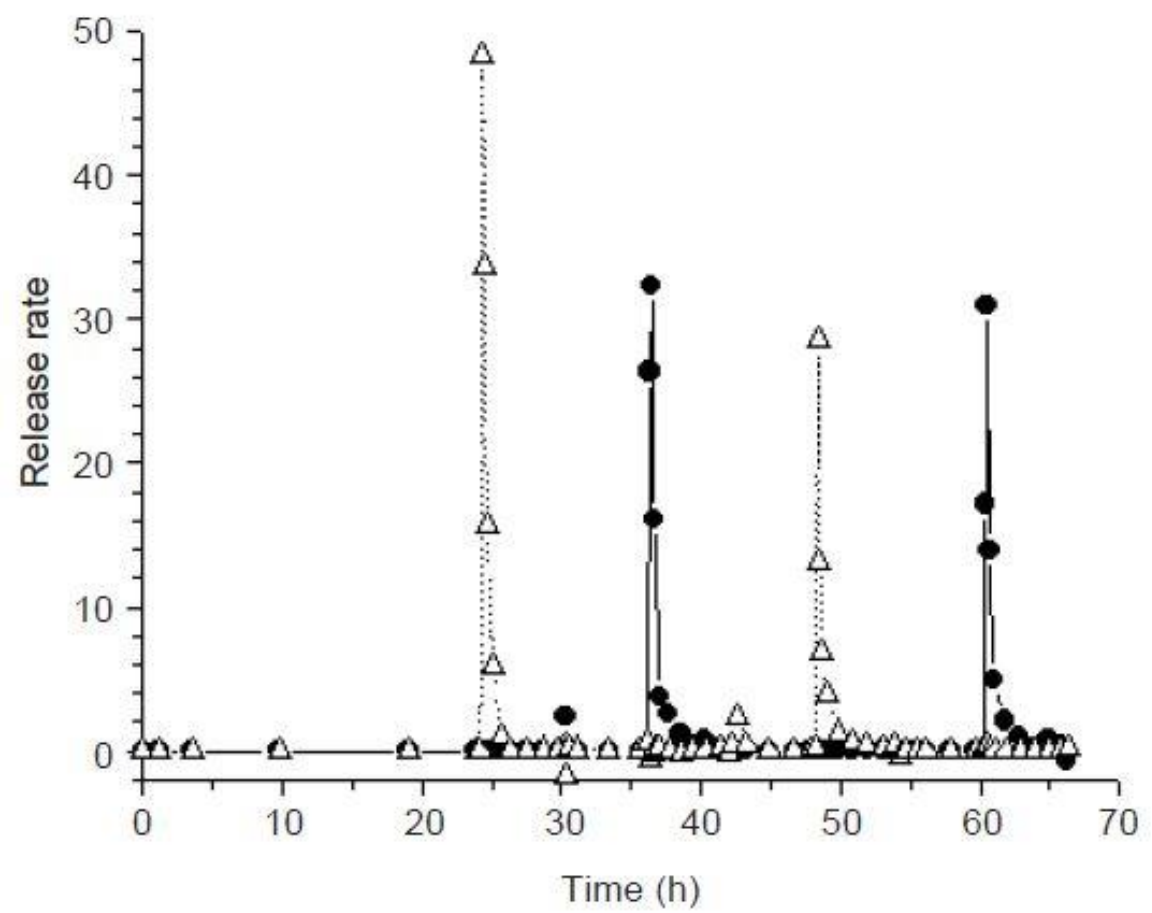

$0.5 \mathrm{~V}$ for 10 second every 5 minutes [81].

Figure 2.20. On-demand multi-drug release from a single microchip device. The release rate of calcium ions $(\Delta)$ sodium fluorescein $(\bullet)$. (Release rate unit is $\mathrm{ng} / \mathrm{min}$ ) [48].

\subsection{Conclusions and future perspectives}

Electrically-responsive biomaterials for targeted drug delivery have gained the interest of scientists in recent years. Applying an electric field to skin and tissue can 
enhance membrane permeability, which can enhance absorption of drug. Moreover, this application is beneficial for delivering large molecules to tissue that normally exhibit poor absorption. The aforementioned biomaterials are biocompatible and have similar mechanical properties with surrounding tissue, which can minimize the foreign body response. By carefully selecting the hydrogel material and its properties, gels can be designed that incorporate drug(s) within their network. Additionally, by controlling morphology of these systems (mesh size and porosity), adding conductive materials, and controlling the electric field intensity and polarity, a desired quantity of drug released can be achieved without damaging the surrounding environment. Additionally, recent studies demonstrate that hydrogel systems can be engineered in a manner where multiple drugs can be delivered to the tissue.

Wireless technologies have the potential to overcome invasiveness and the risk of necrosis while enabling on-demand multi-drug delivery. Conductive hydrogels are a promising technology that have great potential for drug delivery to deep tissue, which currently poses a great challenge to scientists. Hydrogel matrices can be engineered to release drugs using a weak electric field density where the electrophysiological activity of body could generate the required electric field. In addition, devices such as a pacemaker or pulse generator can be designed and implanted to generate the required electric field for drug delivery. 


\subsection{References}

[1] A. Weissenfels, M. Teschler, S. Willert, M. Hettchen, M. Frohlich, H. Kleinoder, M. Kohl, S. von Stengel, W. Kemmler, Effects of whole-body electromyostimulation on chronic nonspecific low back pain in adults: a randomized controlled study, J Pain Res 11 (2018) 1949-1957.

[2] S. Hollis, P. McClure, Intramuscular Electrical Stimulation for Muscle Activation of the Tibialis Anterior After Surgical Repair: A Case Report, J Orthop Sports Phys Ther 47(12) (2017) 965-969.

[3] L. Kapural, N. Mekhail, S.M. Hayek, M. Stanton-Hicks, O. Malak, Occipital nerve electrical stimulation via the midline approach and subcutaneous surgical leads for treatment of severe occipital neuralgia: a pilot study, Anesth Analg 101(1) (2005) 171-4, table of contents.

[4] J.M. Bjordal, M.I. Johnson, A.E. Ljunggreen, Transcutaneous electrical nerve stimulation (TENS) can reduce postoperative analgesic consumption. A metaanalysis with assessment of optimal treatment parameters for postoperative pain, European Journal of Pain 7(2) (2003) 181-188.

[5] W. Rongies, J. Bojakowski, L. Koktysz, W. Dolecki, A.O. Lewinska, A. Krzeski, Physiotherapy in postinfection injury to cranial nerves III, IV, and VI: a case study, Am J Phys Med Rehabil (2018).

[6] T. Kitamura, N. Mizukami, H. Mizoguchi, S. Sakaino, T. Tsuji, Control with Adjusted Pulse Frequency and Amplitude in Functional Electrical Stimulation, Conf Proc IEEE Eng Med Biol Soc 2018 (2018) 2064-2067. 
[7] Y. Watanabe, N.A.R. Nik-Mohd-Afizan, I. Takashima, Blood-brain barrier derangement after electrical brain stimulation, Journal of neurology and neuromedicin 2(10) (2017) 1-5.

[8] M.L. Audu, L.M. Lombardo, J.R. Schnellenberger, K.M. Foglyano, M.E. Miller, R.J. Triolo, A neuroprosthesis for control of seated balance after spinal cord injury, Journal of NeuroEngineering and Rehabilitation 12(1) (2015) 8.

[9] L.K. Lepley, E.M. Wojtys, R.M. Palmieri-Smith, Combination of eccentric exercise and neuromuscular electrical stimulation to improve quadriceps function post-ACL reconstruction, Knee 22(3) (2015) 270-7.

[10] E. Koutsioumpa, D. Makris, A. Theochari, D. Bagka, S. Stathakis, E. Manoulakas, M. Sgantzos, E. Zakynthinos, Effect of Transcutaneous Electrical Neuromuscular Stimulation on Myopathy in Intensive Care Patients, Am J Crit Care 27(6) (2018) 495-503.

[11] G. Torkaman, Electrical Stimulation of Wound Healing: A Review of Animal Experimental Evidence, Adv Wound Care (New Rochelle) 3(2) (2014) 202218.

[12] G. Thakral, J. Lafontaine, B. Najafi, T.K. Talal, P. Kim, L.A. Lavery, Electrical stimulation to accelerate wound healing, Diabet Foot Ankle 4 (2013).

[13] R.R. Isseroff, S.E. Dahle, Electrical Stimulation Therapy and Wound Healing: Where Are We Now?, Adv Wound Care (New Rochelle) 1(6) (2012) 238-243.

[14] Y.D. Choi, J.H. Lee, Edema and pain reduction using transcutaneous electrical nerve stimulation treatment, J Phys Ther Sci 28(11) (2016) 3084-3087. 
[15] A.R. Snyder, A.L. Perotti, K.C. Lam, R.C. Bay, The influence of high-voltage electrical stimulation on edema formation after acute injury: a systematic review, J Sport Rehabil 19(4) (2010) 436-51.

[16] I.O. Man, M.C. Morrissey, J.K. Cywinski, Effect of neuromuscular electrical stimulation on ankle swelling in the early period after ankle sprain, Phys Ther 87(1) (2007) 53-65.

[17] S. Michlovitz, W. Smith, M. Watkins, Ice and High Voltage Pulsed Stimulation in Treatment of Acute Lateral Ankle Sprains, Journal of Orthopaedic \& Sports Physical Therapy 9(9) (1988) 301-304.

[18] Y. Brudno, D.J. Mooney, On-demand drug delivery from local depots, J Control Release 219 (2015) 8-17.

[19] T.M. Herrington, J.J. Cheng, E.N. Eskandar, Mechanisms of deep brain stimulation, J Neurophysiol 115(1) (2016) 19-38.

[20] T. Yan, F. Zong, X. Ma, X. Xu, W. Chen, Z. Song, X. Han, X. Wang, H. Zhang, Cochlear implantation in patients with ossified cochleas, Am J Otolaryngol (2018).

[21] J.A. Chikar, J.L. Hendricks, S.M. Richardson-Burns, Y. Raphael, B.E. Pfingst, D.C. Martin, The use of a dual PEDOT and RGD-functionalized alginate hydrogel coating to provide sustained drug delivery and improved cochlear implant function, Biomaterials 33(7) (2012) 1982-90.

[22] Y.H. Yun, B.K. Lee, K. Park, Controlled Drug Delivery: Historical perspective for the next generation, J Control Release 219 (2015) 2-7. 
[23] S. Sershen, J. West, Implantable, polymeric systems for modulated drug delivery, Advanced Drug Delivery Reviews 54(9) (2002) 1225-1235.

[24] M.C. Koetting, J.T. Peters, S.D. Steichen, N.A. Peppas, Stimulus-responsive hydrogels: Theory, modern advances, and applications, Mater Sci Eng R Rep 93 (2015) 1-49.

[25] J. Qu, X. Zhao, P.X. Ma, B. Guo, pH-responsive self-healing injectable hydrogel based on N-carboxyethyl chitosan for hepatocellular carcinoma therapy, Acta Biomater 58 (2017) 168-180.

[26] G. Jeon, S.Y. Yang, J. Byun, J.K. Kim, Electrically actuatable smart nanoporous membrane for pulsatile drug release, Nano Lett 11(3) (2011) 1284-8.

[27] N. Hosseini-Nassab, D. Samanta, Y. Abdolazimi, J.P. Annes, R.N. Zare, Electrically controlled release of insulin using polypyrrole nanoparticles, Nanoscale 9(1) (2017) 143-149.

[28] J. Qu, X. Zhao, P.X. Ma, B. Guo, Injectable antibacterial conductive hydrogels with dual response to an electric field and pH for localized "smart" drug release, Acta Biomater 72 (2018) 55-69.

[29] Y. Liu, A. Servant, O.J. Guy, K.T. Al-Jamal, P.R. Williams, K.M. Hawkins, K. Kostarelos, An electric-field responsive microsystem for controllable miniaturised drug delivery applications, Sensors and Actuators B: Chemical 175 (2012) 100-105.

[30] L.M. Lira, S.I. Córdoba de Torresi, Conducting polymer-hydrogel composites for electrochemical release devices: Synthesis and characterization of semi- 
interpenetrating polyaniline-polyacrylamide networks, Electrochemistry Communications 7(7) (2005) 717-723.

[31] D. Zhang, F. Di, Y. Zhu, Y. Xiao, J. Che, Electroactive hybrid hydrogel: Toward a smart coating for neural electrodes, Journal of Bioactive and Compatible Polymers 30(6) (2015) 600-616.

[32] N. Paradee, A. Sirivat, S. Niamlang, W. Prissanaroon-Ouajai, Effects of crosslinking ratio, model drugs, and electric field strength on electrically controlled release for alginate-based hydrogel, J Mater Sci Mater Med 23(4) (2012) 999-1010.

[33] J. Sittiwong, S. Niamlang, N. Paradee, A. Sirivat, Electric field-controlled benzoic acid and sulphanilamide delivery from poly(vinyl alcohol) hydrogel, AAPS PharmSciTech 13(4) (2012) 1407-15.

[34] S. Pairatwachapun, N. Paradee, A. Sirivat, Controlled release of acetylsalicylic acid from polythiophene/carrageenan hydrogel via electrical stimulation, Carbohydr Polym 137 (2016) 214-221.

[35] K. Krukiewicz, P. Zawisza, A.P. Herman, R. Turczyn, S. Boncel, J.K. Zak, An electrically controlled drug delivery system based on conducting poly $(3,4-$ ethylenedioxypyrrole) matrix, Bioelectrochemistry 108 (2016) 13-20.

[36] D. Mawad, A. Lauto, G.G. Wallace, Conductive Polymer Hydrogels, in: S. Kalia (Ed.), Polymeric Hydrogels as Smart Biomaterials, Springer International Publishing, Cham, 2016, pp. 19-44. 
[37] G. Kiaee, P. Mostafalu, M. Samandari, S. Sonkusale, A pH-Mediated Electronic Wound Dressing for Controlled Drug Delivery, Adv Healthc Mater (2018) e1800396.

[38] C.J. Pérez-Martínez, S.D. Morales Chávez, T. del Castillo-Castro, T.E. Lara Ceniceros, M.M. Castillo-Ortega, D.E. Rodríguez-Félix, J.C. Gálvez Ruiz, Electroconductive nanocomposite hydrogel for pulsatile drug release, Reactive and Functional Polymers 100 (2016) 12-17.

[39] L. Wu, J. Wang, N. Gao, J. Ren, A. Zhao, X. Qu, Electrically pulsatile responsive drug delivery platform for treatment of Alzheimer's disease, Nano Research 8(7) (2015) 2400-2414.

[40] M.P. Rols, Electropermeabilization, a physical method for the delivery of therapeutic molecules into cells, Biochim Biophys Acta 1758(3) (2006) 423-8.

[41] F. Fantozzi, E. Arturoni, R. Barbucci, The effects of the electric fields on hydrogels to achieve antitumoral drug release, Bioelectrochemistry 78(2) (2010) 191-5.

[42] S.J. Kim, S.G. Yoon, S.M. Lee, J.H. Lee, S.I. Kim, Characteristics of electrical responsive alginate/poly(diallyldimethylammonium chloride) IPN hydrogel in HCl solutions, Sensors and Actuators B: Chemical 96(1-2) (2003) 1-5.

[43] S. Dubey, Y.N. Kalia, Understanding the poor iontophoretic transport of lysozyme across the skin: when high charge and high electrophoretic mobility are not enough, J Control Release 183 (2014) 35-42. 
[44] S. Kantaria, G.D. Rees, M.J. Lawrence, Gelatin-stabilised microemulsion-based organogels: rheology and application in iontophoretic transdermal drug delivery, Journal of Controlled Release 60(2-3) (1999) 355-365.

[45] G. Schmidt, I. Juhasz-Boss, E.F. Solomayer, D. Herr, Electrochemotherapy in Breast Cancer: A Review of References, Geburtshilfe Frauenheilkd 74(6) (2014) 557-562.

[46] M.R. Prausnitz, V.G. Bose, R. Langer, J.C. Weaver, Electroporation of mammalian skin: a mechanism to enhance transdermal drug delivery, Proceedings of the National Academy of Sciences 90(22) (1993) 10504-10508.

[47] X. Ying, Y. Wang, J. Liang, J. Yue, C. Xu, L. Lu, Z. Xu, J. Gao, Y. Du, Z. Chen, Angiopep-Conjugated Electro-Responsive Hydrogel Nanoparticles: Therapeutic Potential for Epilepsy, Angewandte Chemie 126(46) (2014) $12644-12648$.

[48] J.T. Santini Jr, M.J. Cima, R. Langer, A controlled-release microchip, Nature 397 (1999) 335 .

[49] J.M. Maloney, S.A. Uhland, B.F. Polito, N.F. Sheppard, Jr., C.M. Pelta, J.T. Santini, Jr., Electrothermally activated microchips for implantable drug delivery and biosensing, J Control Release 109(1-3) (2005) 244-55.

[50] R. Farra, N.F. Sheppard, Jr., L. McCabe, R.M. Neer, J.M. Anderson, J.T. Santini, Jr., M.J. Cima, R. Langer, First-in-human testing of a wirelessly controlled drug delivery microchip, Sci Transl Med 4(122) (2012) 122ra21.

[51] Y.T. Yi, J.Y. Sun, Y.W. Lu, Y.C. Liao, Programmable and on-demand drug release using electrical stimulation, Biomicrofluidics 9(2) (2015) 022401. 
[52] P. Chansai, A. Sirivat, S. Niamlang, D. Chotpattananont, K. Viravaidya-Pasuwat, Controlled transdermal iontophoresis of sulfosalicylic acid from polypyrrole/poly(acrylic acid) hydrogel, Int J Pharm 381(1) (2009) 25-33.

[53] V. Guarino, S. Zuppolini, A. Borriello, L. Ambrosio, Electro-Active Polymers (EAPs): A Promising Route to Design Bio-Organic/Bioinspired Platforms with on Demand Functionalities, Polymers 8(5) (2016).

[54] S. Inal, J. Rivnay, A.O. Suiu, G.G. Malliaras, I. McCulloch, Conjugated Polymers in Bioelectronics, Acc Chem Res 51(6) (2018) 1368-1376.

[55] P. Minhas, V. Bansal, J. Patel, J.S. Ho, J. Diaz, A. Datta, M. Bikson, Electrodes for high-definition transcutaneous DC stimulation for applications in drug delivery and electrotherapy, including tDCS, J Neurosci Methods 190(2) (2010) 188-97.

[56] A. Servant, L. Methven, R.P. Williams, K. Kostarelos, Electroresponsive polymer-carbon nanotube hydrogel hybrids for pulsatile drug delivery in vivo, Adv Healthc Mater 2(6) (2013) 806-11.

[57] A. Gasselhuber, M.R. Dreher, A. Negussie, B.J. Wood, F. Rattay, D. Haemmerich, Mathematical spatio-temporal model of drug delivery from low temperature sensitive liposomes during radiofrequency tumour ablation, Int J Hyperthermia 26(5) (2010) 499-513.

[58] F. Yan, S. Wang, W. Yang, S.N. Goldberg, H. Wu, W.L. Duan, Z.T. Deng, H.B. Han, H.R. Zheng, Tumor-penetrating Peptide-integrated Thermally Sensitive Liposomal Doxorubicin Enhances Efficacy of Radiofrequency Ablation in Liver Tumors, Radiology 285(2) (2017) 462-471. 
[59] C. Rossmann, M.A. McCrackin, K.E. Armeson, D. Haemmerich, Temperature sensitive liposomes combined with thermal ablation: Effects of duration and timing of heating in mathematical models and in vivo, PLoS One 12(6) (2017) e0179131.

[60] Y. Osada, H. Okuzaki, H. Hori, A polymer gel with electrically driven motility, Nature 355 (1992) 242.

[61] R. Tomer, D. Dimitrijevic, A.T. Florence, Electrically controlled release of macromolecules from cross-linked hyaluronic acid hydrogels, Journal of Controlled Release 33(3) (1995) 405-413.

[62] S. Ganguly, D. Ray, P. Das, P.P. Maity, S. Mondal, V.K. Aswal, S. Dhara, N.C. Das, Mechanically robust dual responsive water dispersible-graphene based conductive elastomeric hydrogel for tunable pulsatile drug release, Ultrason Sonochem 42 (2018) 212-227.

[63] X. Jin, H. Kang, Y. Huang, H. Liu, Y. Hu, Synthesis and properties of electrically-sensitive poly(acrylic acid-co-acetoacetoxy ethyl methacrylate) gels, Journal of Applied Polymer Science 110(6) (2008) 3690-3696.

[64] S. Kennedy, S. Bencherif, D. Norton, L. Weinstock, M. Mehta, D. Mooney, Rapid and extensive collapse from electrically responsive macroporous hydrogels, Adv Healthc Mater 3(4) (2014) 500-7.

[65] T. Shiga, T. Kurauchi, Deformation of polyelectrolyte gels under the influence of electric field, Journal of Applied Polymer Science 39(1112) (1990) 2305-2320. 
[66] S. Ramanathan, L.H. Block, The use of chitosan gels as matrices for electricallymodulated drug delivery, Journal of Controlled Release 70(1-2) (2001) 109123.

[67] I.C. Kwon, Y.H. Bae, S.W. Kim, Electrically erodible polymer gel for controlled release of drugs, Nature 354(6351) (1991) 291-3.

[68] S. Ganguly, P. Das, P.P. Maity, S. Mondal, S. Ghosh, S. Dhara, N.C. Das, Green Reduced Graphene Oxide Toughened Semi-IPN Monolith Hydrogel as Dual Responsive Drug Release System: Rheological, Physicomechanical, and Electrical Evaluations, J Phys Chem B 122(29) (2018) 7201-7218.

[69] G. Liu, X. Zhao, Electroresponsive Behavior of Gelatin/Alginate Semi-interpenetrating Polymer Network Membranes Under Direct-Current Electric Field, Journal of Macromolecular Science, Part A 43(2) (2007) 345354.

[70] S.Y. Kim, Y.M. Lee, Drug release behavior of electrical responsive poly(vinyl alcohol)/poly(acrylic acid) IPN hydrogels under an electric stimulus, Journal of Applied Polymer Science 74(7) (1999) 1752-1761.

[71] C.S. Hsu, L.H. Block, Anionic gels as vehicles for electrically-modulated drug delivery. solvent and drug transport phenomena, Pharmaceutical Research 13(12) (1996) 1865-1870.

[72] J. Lin, Q. Tang, D. Hu, X. Sun, Q. Li, J. Wu, Electric field sensitivity of conducting hydrogels with interpenetrating polymer network structure, Colloids and Surfaces A: Physicochemical and Engineering Aspects 346(1-3) (2009) 177-183. 
[73] U.G. Spizzirri, S. Hampel, G. Cirillo, F.P. Nicoletta, A. Hassan, O. Vittorio, N. Picci, F. Iemma, Spherical gelatin/CNTs hybrid microgels as electroresponsive drug delivery systems, Int J Pharm 448(1) (2013) 115-22.

[74] G. Filipcsei, J. Fehér, M. Zrínyi, Electric field sensitive neutral polymer gels, Journal of Molecular Structure 554(1) (2000) 109-117.

[75] W. Sangwan, K. Petcharoen, N. Paradee, W. Lerdwijitjarud, A. Sirivat, Electrically responsive materials based on polycarbazole/sodium alginate hydrogel blend for soft and flexible actuator application, Carbohydr Polym 151 (2016) 213-222.

[76] J.M. Miller, R.C. de Guzman, PABA Release from Chitosan-PCL with Induced Electric Current, bioRxiv (2018) 377481.

[77] J.S. Im, B. Bai, Y.S. Lee, The effect of carbon nanotubes on drug delivery in an electro-sensitive transdermal drug delivery system, Biomaterials 31(6) (2010) 1414-9.

[78] M.R. Abidian, D.H. Kim, D.C. Martin, Conducting-Polymer Nanotubes for Controlled Drug Release, Adv Mater 18(4) (2006) 405-409.

[79] N. Paradee, A. Sirivat, Electrically controlled release of benzoic acid from poly(3,4-ethylenedioxythiophene)/alginate matrix: effect of conductive poly(3,4-ethylenedioxythiophene) morphology, J Phys Chem B 118(31) (2014) 9263-71.

[80] C. Chen, X. Chen, H. Zhang, Q. Zhang, L. Wang, C. Li, B. Dai, J. Yang, J. Liu, D. Sun, Electrically-responsive core-shell hybrid microfibers for controlled drug release and cell culture, Acta Biomater 55 (2017) 434-442. 
[81] J. Ge, E. Neofytou, T.J. Cahill, 3rd, R.E. Beygui, R.N. Zare, Drug release from electric-field-responsive nanoparticles, ACS Nano 6(1) (2012) 227-33.

[82] Y. Wu, Y.X. Chen, J. Yan, D. Quinn, P. Dong, S.W. Sawyer, P. Soman, Fabrication of conductive gelatin methacrylate-polyaniline hydrogels, Acta Biomater 33 (2016) 122-30.

[83] B. Guo, L. Glavas, A.-C. Albertsson, Biodegradable and electrically conducting polymers for biomedical applications, Progress in Polymer Science 38(9) (2013) 1263-1286.

[84] S. Niamlang, A. Sirivat, Electrically controlled release of salicylic acid from poly(p-phenylene vinylene)/polyacrylamide hydrogels, Int J Pharm 371(1-2) (2009) 126-33.

[85] N. Mac Kenna, P. Calvert, A. Morrin, G.G. Wallace, S.E. Moulton, Electrostimulated release from a reduced graphene oxide composite hydrogel, Journal of Materials Chemistry B 3(12) (2015) 2530-2537.

[86] G. Sharifzadeh, H. Hosseinkhani, Biomolecule-Responsive Hydrogels in Medicine, Adv Healthc Mater 6(24) (2017).

[87] S. Naficy, J.M. Razal, G.M. Spinks, G.G. Wallace, P.G. Whitten, Electrically Conductive, Tough Hydrogels with pH Sensitivity, Chemistry of Materials 24(17) (2012) 3425-3433.

[88] E.J. Choi, J. Shin, Z.H. Khaleel, I. Cha, S.-H. Yun, S.-W. Cho, C. Song, Synthesis of electroconductive hydrogel films by an electro-controlled click reaction and their application to drug delivery systems, Polymer Chemistry 6(24) (2015) 4473-4478. 
[89] A. Servant, V. Leon, D. Jasim, L. Methven, P. Limousin, E.V. FernandezPacheco, M. Prato, K. Kostarelos, Graphene-based electroresponsive scaffolds as polymeric implants for on-demand drug delivery, Adv Healthc Mater 3(8) (2014) 1334-43.

[90] Y. Xiang, D. Chen, Preparation of a novel pH-responsive silver nanoparticle/poly(HEMA-PEGMA-MAA) composite hydrogel, European Polymer Journal 43(10) (2007) 4178-4187.

[91] L.X. Gao, J.L. Chen, X.W. Han, S.X. Yan, Y. Zhang, W.Q. Zhang, Z.W. Gao, Electro-response characteristic of starch hydrogel crosslinked with Glutaraldehyde, J Biomater Sci Polym Ed 26(9) (2015) 545-57.

[92] K. Juntanon, S. Niamlang, R. Rujiravanit, A. Sirivat, Electrically controlled release of sulfosalicylic acid from crosslinked poly(vinyl alcohol) hydrogel, Int J Pharm 356(1-2) (2008) 1-11.

[93] S. Indermun, Y.E. Choonara, P. Kumar, L.C. du Toit, G. Modi, R. Luttge, V. Pillay, An interfacially plasticized electro-responsive hydrogel for transdermal electro-activated and modulated (TEAM) drug delivery, Int J Pharm 462(1-2) (2014) 52-65.

[94] R.V. Kulkarni, B. Sa, Electroresponsive Polyacrylamide-grafted-xanthan Hydrogels for Drug Delivery, Journal of Bioactive and Compatible Polymers 24(4) (2009) 368-384.

[95] S. Mongkolkitikul, N. Paradee, A. Sirivat, Electrically controlled release of ibuprofen from conductive poly(3-methoxydiphenylamine)/crosslinked pectin hydrogel, Eur J Pharm Sci 112 (2018) 20-27. 
[96] K.H. Liu, T.Y. Liu, S.Y. Chen, D.M. Liu, Drug release behavior of chitosanmontmorillonite nanocomposite hydrogels following electrostimulation, Acta Biomater 4(4) (2008) 1038-45.

[97] Q. Wu, J. Wei, B. Xu, X. Liu, H. Wang, W. Wang, Q. Wang, W. Liu, A robust, highly stretchable supramolecular polymer conductive hydrogel with selfhealability and thermo-processability, Sci Rep 7 (2017) 41566.

[98] M. Chen, Y.F. Li, F. Besenbacher, Electrospun nanofibers-mediated on-demand drug release, Adv Healthc Mater 3(11) (2014) 1721-32.

[99] X. Shi, Y. Zheng, C. Wang, L. Yue, K. Qiao, G. Wang, L. Wang, H. Quan, Dual stimulus responsive drug release under the interaction of $\mathrm{pH}$ value and pulsatile electric field for a bacterial cellulose/sodium alginate/multi-walled carbon nanotube hybrid hydrogel, RSC Advances 5(52) (2015) 41820-41829.

[100] T. Tungkavet, N. Seetapan, D. Pattavarakorn, A. Sirivat, Graphene/gelatin hydrogel composites with high storage modulus sensitivity for using as electroactive actuator: Effects of surface area and electric field strength, Polymer 70 (2015) 242-251.

[101] T. Dai, X. Qing, Y. Lu, Y. Xia, Conducting hydrogels with enhanced mechanical strength, Polymer 50(22) (2009) 5236-5241.

[102] W. Li, X. Zeng, H. Wang, Q. Wang, Y. Yang, Polyaniline-poly(styrene sulfonate) conducting hydrogels reinforced by supramolecular nanofibers and used as drug carriers with electric-driven release, European Polymer Journal 66 (2015) 513-519. 
[103] S.M. Richardson-Burns, J.L. Hendricks, B. Foster, L.K. Povlich, D.H. Kim, D.C. Martin, Polymerization of the conducting polymer poly $(3,4-$ ethylenedioxythiophene) (PEDOT) around living neural cells, Biomaterials 28(8) (2007) 1539-52.

[104] H. Jo, M. Sim, S. Kim, S. Yang, Y. Yoo, J.H. Park, T.H. Yoon, M.G. Kim, J.Y. Lee, Electrically conductive graphene/polyacrylamide hydrogels produced by mild chemical reduction for enhanced myoblast growth and differentiation, Acta Biomater 48 (2017) 100-109.

[105] D.H. Kim, M. Abidian, D.C. Martin, Conducting polymers grown in hydrogel scaffolds coated on neural prosthetic devices, J Biomed Mater Res A 71(4) (2004) 577-85.

[106] X. Zhou, Y.C. Hon, S. Sun, A.F.T. Mak, Numerical simulation of the steadystate deformation of a smart hydrogel under an external electric field, Smart Materials and Structures 11(3) (2002) 459-467.

[107] S. Niamlang, T. Buranut, A. Niansiri, Electrically Controlled Aloe-Vera Extraction Release from Poly Acrylamide Hydrogel, Energy Procedia 9 (2011) 468-473.

[108] S. Lee, J. Lee, Y.W. Choi, Skin Permeation Enhancement of Ascorbyl Palmitate by Liposomal Hydrogel (Lipogel) Formulation and Electrical Assistance, Biological \& Pharmaceutical Bulletin 30(2) (2007) 393-396.

[109] G. Bianchi, L. Campanacci, M. Ronchetti, D. Donati, Electrochemotherapy in the Treatment of Bone Metastases: A Phase II Trial, World J Surg 40(12) (2016) 3088-3094. 
[110] J. Kulsh, Targeting a key enzyme in cell growth: a novel therapy for cancer, Medical Hypotheses 49(4) (1997) 297-300.

[111] Z. Shi, Y. Li, X. Chen, H. Han, G. Yang, Double network bacterial cellulose hydrogel to build a biology-device interface, Nanoscale 6(2) (2014) 970-7.

[112] J. Xi, X.A. Si, R. Gaide, Electrophoretic particle guidance significantly enhances olfactory drug delivery: a feasibility study, PLoS One 9(1) (2014) e86593.

[113] X. Luo, X.T. Cui, Sponge-like nanostructured conducting polymers for electrically controlled drug release, Electrochem commun 11(10) (2009) 1956. 


\section{CHAPTER 3}

Cryogel-Based Electronic-Tissue Interfaces with Soft, Highly Compressible, and Tunable Mechanics

Submitted to Advanced Health Care Materials

Rosa Ghatee ${ }^{1}$, Anita Tolouei ${ }^{1}$, Jennifer Fijalkowski ${ }^{1}$, Abdulrahman Alsasa ${ }^{2}$, Justin Hayes $^{1}$, Walter Besio ${ }^{2}$, Stephen Kennedy ${ }^{1,2}$

${ }^{1}$ Department of Chemical Engineering, ${ }^{2}$ Department of Electrical, Computer and Biomedical Engineering, University of Rhode Island, Kingston, Rhode Island 02881, U.S.A. 


\section{Abstract}

Electrically conductive materials with soft, tough, and tunable mechanics have utility in a wide range of applications including neuroprosthetics. Such materials can serve as interfaces between electrical components and tissues, providing mechanical matches with, and better conformations to, soft irregularly shaped surfaces. Hydrogels can potentially provide these attributes while remaining hydrated for long periods of time-providing a long-term and stable electronic-tissue interface. Additionally, in applications that demand implantation, hydrogels can be formulated to locally deliver enhancing therapeutics. Here, hydrogels are developed by entrapping a conducting polymer within a crosslinked poly(acrylic acid) (pAAc) network. Critically, these hydrogels were cast under freezing conditions which produces cryogels that exhibit macroporous, soft, and highly tunable mechanics $(0.2-20 \mathrm{kPa}$, by varying pAAc and crosslinker concentrations). Additionally, these cryogels are tough enough to survive over $90 \%$ compression, which enables survival after being passed through 16-gauge needles. Cryogels also exhibit electrical conductivities that were sufficient to record alpha-waves from the scalp of human subjects. Growth of fibroblasts cultures in the presence of these cryogels produce statistically similar viabilities compared to controls and did not disrupt fibroblast cell cycles. Finally, cryogels are capable of being loaded with and delivering proteins that can combat inflammation.

Keywords: hydrogels, electrodes, conductive polymers, neuroprosthetic 


\subsection{Introduction}

Electrically conductive materials that exhibit soft, deformable, but tough mechanical properties are highly desirable in a wide range of applications. For example, such materials could enhance neurological disorder treatment strategies - a family of disorders that affect up to a billion people worldwide and are on the rise due to gradual increases in life expectancies [1]. Neuroprosthetic devices that can restore functionality or reduce the symptoms exhibited by patients suffering from neurological diseases often require the use of electrodes to stimulate and/or record signals from neural tissues [2]. For instance, deep brain stimulation (DBS) uses implanted electrodes to electrically stimulate targets within the brain [3,4], which can reduce symptoms exhibited by patients with Parkinson's disease, essential tremor, dystonia, chronic pain, major depression, and obsessive-compulsive disorder [5]. Such strategies require an electrode-tissue interface whose electrical conductivity remains sufficiently high to transmit neural signals over time [6]. Metallic electrodes are typically used as the electrode material in these neuroprosthetic applications due to their high electrical conductivity. However, the stiffness of metallic electrodes can be problematic, especially in applications that require prolonged electrode-tissue interface and/or applications that demand electrode implantation within tissues. Specifically, stiff electrodes can have difficulty remaining in contact with soft, curvilinear surfaces (e.g., against the skin in electroencephalography (EEG) and electrocardiography (ECG) applications). Conductive pastes (such as Ten20 Conductive EEG Paste) are typically used to as interfaces to help facilitate a stable electrical pathway between 
electrodes and soft surface tissues. However, they involve messy and time-consuming electrode preparation before use, dry up during use, and are therefore not well-suited for applications requiring recording/stimulating over prolonged periods of time (e.g., for wearable sensors and electronics). For applications that demand in vivo electrode implantation (e.g., DBS), maintenance of an electrically conductive path across the electrode-tissue interface is further complicated by the body's ability to build fibrous tissue around the implanted electrode, which can greatly reduce interfacial conductivity $[2,7-9]$. Fibrous tissue development at the electrode-tissue interface is thought to be the result of several factors. First, surgical introduction of a stiff metal electrode to sensitive tissues can be traumatic and can incite a strong inflammatory response. As time progresses, the mechanical mismatch between the stiff metallic electrode and soft tissue can lead to micromotion, shear stresses, continued inflammation, and a stronger foreign body reaction $[2,8]$. These responses can lead to accelerated fibrous capsule formation around the electrode, thereby accelerating the loss of the requisite electrically conductive path. Because of the issues outlined above, this work aimed to develop a versatile material for interfacing between electronics and tissues that exhibited (i) soft mechanics to help facilitate conformation to curvilinear surfaces and to provide better mechanical matches with native tissues, (ii) sufficient electrical conductivity to record low-powered neural signals, (iii) tunable mechanics to enable application-specific customizations, (iv) toughness and compressibility to enable less traumatic introduction to tissues via injection, (v) low cytotoxicity, and (vi) the ability to locally deliver therapeutics that could potentially aid in combatting inflammation and the foreign body reaction. 
In order to achieve the above-listed properties, a hydrogel-based material was pursued. Hydrogels are soft materials made from cross-linked hydrophilic polymer networks [10] and have the potential to locally deliver therapeutic agents such as antiinflammatories [11]. Hydrogels can also have similar mechanical properties as physiological tissues [12]. However, they normally exhibit poor electrical conductivity [13] making them inappropriate for use as neuroprosthetic-tissue interface materials [14-16]. Electrically conductive polymer hydrogels are a unique class of hydrogels that not only can have similar mechanical properties as neural tissue $[17,18]$ but can also have electrical properties similar to metals and inorganic semiconductors.[19] It has previously been demonstrated that hydrogels composed of poly(3-4, ethylenedioxythiophene) (PEDOT) provide electrical conductivity and stability in oxidized environments [13, 20-24]. Moreover, PEDOT hydrogels in particular are biocompatible [25] and can have similar Young's moduli as neural tissues [2]. For these reasons, PEDOT was employed as the conductive component of the hydrogelbased interface material described here. 


\subsection{Experimental Section}

\subsubsection{PEDOT/pAAc hydrogel and cryogel preparation:}

Different amounts of acrylic acid (AAc), $550 \mathrm{Da}$ Poly (ethylene glycol) Dimethacrylate (PEGDM), sodium hydroxide $(\mathrm{NaOH})$ (465 $\mu \mathrm{g}$ per $\mathrm{ml}$ of AAc), deionized water, and Poly(3,4-ethylenedioxythiophene):polystyrene sulfonate (PEDOT:PSS) were mixed via vortexing. Then N,N,N',N'Tetramethylethylenediamine (TEMED) and Ammonium Persulfate (APS) redox reagents were mixed in to initiate gelation and the solution was immediately transferred to a $10 \times 10 \mathrm{~mm}$ cylindrical Teflon mold. This produced non-macroporous PEDOT/pAAc hydrogels. To produce macroporous cryogels, the same procedure was followed except pre-gel solutions were transferred to frozen Teflon molds (pre-frozen at $-20^{\circ} \mathrm{C}$ ) and left to gel in a $-20^{\circ} \mathrm{C}$ freezer overnight. Both hydrogels and cryogels were rinsed in Phosphate Buffered Saline (PBS) for 3 days (exchanging liquid 3 times daily) prior to running experiments to remove excess reagents and to allow the gels to fully swell.

\subsubsection{Mechanical characterizations:}

Gel stiffness (compressive modulus), strain at failure, and toughness were quantitatively measured in compression using an Instron Model 3345 (Norwood, MA). Gels of various compositions were placed between the plates of the Instron and compressed (at $2 \mathrm{~mm} / \mathrm{min}$ ) until failure or reaching $90 \%$ strain. Note that $90 \%$ compression was never exceeded as to eliminate the risk of damaging the Instron 
should the plates touch during compression. Recorded stress-strain curves were analyzed using the Instron's Bluehill software package to extract moduli, strain at failures, and toughnesses.

\subsubsection{Electrical characterizations:}

For measuring IV-curves and direct current (DC) conductivity, gels of various composition were placed between two stainless-steel plates immediately after swelling in a hydrated state and excited at $0.5,1,2,4$, and 8 VDC. DC current was recorded and used to generate IV-curves. Additionally, DC conductivity was calculated at various voltage excitations using these measured currents along with the measured length and cross-sectional surface area of each fully swollen gel. For measuring impedance vs. frequency, all gels were cut into $12 \mathrm{~mm}$ diameter and $2 \mathrm{~mm}$ height discs after fully swelling. Cut gels of various composition were measured using a GW Instek LCR-6000 meter (New Taipei, Taiwan) at 2 VAC with 2 VDC bias from $10 \mathrm{~Hz}$ to $100 \mathrm{kHz}$.

\subsubsection{EEG studies:}

9 wt \% AAc, 0.26 wt \% PEDOT, 1 wt \% PEGDM cryogels were cast in the 10mm EEG disc electrodes and rinsed/swollen as described above. These PEDOT electrodes were compared to electrodes integrated with Ten20 paste (Weaver and Company, Aurora, CO). After cleaning recording locations on the scalp with a mildly abrasive gel (Nuprep, Weave and Company), PEDOT-, cryoPEDOT, and Ten20integrated electrodes were placed on the back of human subjects' heads over to the 
occipital lobe and held in place with a sweat band. All recordings were in accordance with the University of Rhode Island IRB approved protocol. Reference and ground electrodes were placed on subjects' foreheads. Each subject had one PEDOT-, one cryoPEDOT, and one Ten20-integrated electrode in order to record and compare the same neural signals. Electrodes remained on subjects for 4 hours. EEG signals were recorded after this 4-hour period with subject eyes being closed and opened using an EEG amplifier (Grass Comet-Plus EEG/PSG Amplifier system, Grass EEG systems, Natus Medical Incorporated) and a computer with EEG software (Grass Technologies EEG Twin 4.5.2).

\subsubsection{Gel treatment and injection:}

Only 9 wt \% AAc, 0.26 wt \% PEDOT, 1 wt \% PEGDM gels were used in these studies. Ethanol-treatment of gels involved submerging fully swollen gels in a 70\% ethanol solution for $1 \mathrm{~h}$. In a biosafety cabinet, ethanol-soaked gels were transferred to sterilized PBS (sterilized using a 0.22-micron vacuum filter) and rinsed for 3 days in sterile PBS to remove excess ethanol and to allow the gels to fully reswell (ethanol treatment did cause some degree of gel collapse). Autoclave-sterilization involved placing fully swollen gels in $250 \mathrm{~mL}$ media bottles containing PBS and loosely screwing on the media bottle lids. Gel-containing media bottles were treated in a Tuttanauer 2540 series autoclave (Breda, Netherlands) for 30 minutes at $121^{\circ} \mathrm{C}$. After sterilization, the electrical and mechanical properties of these gels were characterized and compared to gels that were not sterilized using the mechanical and electrical 
testing methods described above. For injectability, fully swollen gels were cut into $3 \mathrm{x}$ $1.25 \mathrm{~mm}$ cylinders and passes through 16-gauge needle with PBS.

\subsubsection{Fibroblast culturing:}

3 T3 mouse fibroblasts were cultured in DMEM with $10 \%$ bovine calf serum and $1 \%$ penicillin streptomycin in $75 \mathrm{~cm}^{2}$ flasks at $37^{\circ} \mathrm{C}$ with $5 \% \mathrm{CO}_{2} .3 \mathrm{~T} 3 \mathrm{~s}$ were routinely passaged to maintain confluency under $70 \%$ by treating with $0.25 \%$ TrypsinEDTA for 10 min, aspirating, centrifugal washing, and re-plating in flaks with fresh growth media.

\subsubsection{Fibroblast studies:}

3 T3 mouse fibroblasts were cultured and plated on 24-well plates at 1000 cells per $\mathrm{cm}$ and allowed to grow for 24 hours before being exposed to cryogels. 9 wt $\%$ AAc, 0.26 wt \% PEDOT, 1 wt \% PEGDM cryogels $(10 \times 10 \mathrm{~mm}$ cylinders $)$ were prepared, rinsed/swollen, ethanol-disinfected (as described above except cryogels were rinsed in sterilized complete DMEM on the $3^{\text {rd }}$ day of post-ethanol rinsing in lieu of sterile PBS), and placed directly on top of 2D fibroblast cultures. After 1, 3, and 7 days of cryogel exposure, cryogels were removed and the 3T3s were analyzed by LIVE/DEAD staining and imaging on a BioTek Cytation 3 plate reader (Winooski, VT). These images were used to calculate cell viability and live cell count vs. time using BioTek Gen5 software.

\subsubsection{Cell cycle analysis:}


fibroblasts were exposed to PEDOT cryogels as described above, but for 10 full days rather than 7 (representing 5 to 6 doubling times). After cryogel exposure fibroblasts were trypsinized, collected, fixed for 15 minutes in ice-cold ethanol, spun down (400 g for 8 minutes), resuspended in a propidium iodide (PI) staining solution (Nexcelom Biosciences), and incubated for 40 minutes at $37^{\circ} \mathrm{C}$. Fibroblasts were again spun down, resuspended in fresh PBS, loaded in to a Nexcelom Cellometer counting chamber, and analyzed on a Nexcelom Cellometer Vision. The Cellometer Vision produced PI intensity histograms and enabled the extraction of the percent of cells in various phases of their cycle, based on relative PI fluorescence intensity, which is proportional to the relative amount of DNA a cell currently has (which indicates what stage the cell is in its cell cycle).

\subsubsection{Protein release studies:}

9 wt \% AAc, 0.26 wt \% PEDOT, 1 wt \% PEGDM cryogels $(10$ x $10 \mathrm{~mm}$ cylinders) were prepared and rinsed/swollen as described above. Cryogels were gently blotted using a Kimwipe to slightly dehydrate them. This slight dehydration allowed for IL-4 to be loaded by adding a concentrated solution of IL-4 (1000 ng and $5000 \mathrm{ng}$ IL-4 in $100 \mu \mathrm{L}$ PBS) dropwise to the gel. IL-4-loaded cryogels were left overnight in a sealed scintillation vial to allow IL-4 to integrate well into the cryogels. These scintillation vials were previously treated with Sigmacote to prevent binding of released protein to the walls of the scintillation vial. Cryogels were then submerged in $5 \mathrm{~mL}$ of PBS with $1 \%$ Bovine Serum Albumen (BSA). Over the course of a month, 5 
$\mathrm{mL}$ samples were taken and replaced with fresh $5 \mathrm{~mL}$ of PBS with $1 \%$ BSA. These samples were analyzed for IL-4 content using ELISA (R \& D Systems).

\subsubsection{Sample preparation for SEM imaging:}

9 wt $\%$ AAc, 0.26 wt \% PEDOT, 1 wt \% PEGDM cryogels $(10$ x $10 \mathrm{~mm}$ cylinders) were prepared as described in the Experimental section but lyophilized for 3 days instead of being thawed and rinsed. After lyophilization, dehydrated cryogels were cut in half using a sharp razor to expose the vertical cross-section of the gel. The cross-cut cryogel was sputter-coated with gold for 30 seconds, mounted, and placed in the imaging chamber of a Zeiss SIGMA VP Field Emissions Scanning Electron Microscope (FE-SEM).

3.2.11 Data representation and statistical analyses: All quantitative data presented in this work are represented as means \pm standard deviations. For statistical comparisons that were limited to single comparisons, a student t-test was applied to calculate significance with $p$-values of less than 0.05 being our benchmark for significance. For instances where multiple comparisons were made across multiple conditions, one-way Analysis of Variance (ANOVA) with a Tukey's post hoc test was utilized. The

following convention was used for indicating the level of significance: *,**, ***, and $* * * *$ indicate $p<0.05,0.01,0.001$, and 0.0001 respectively. "n.s." indicates that this benchmark for statistical significance was not met $(p>0.05)$. 


\subsection{Results and Discussion}

PEDOT hydrogels were generated by entrapping PEDOT polymer within poly(acrylic acid) (pAAc) matrices using 550 Da poly(ethylene glycol) dimethacrylate (PEGDM) as a crosslinker (see Experimental Section for more details). A wide variety of hydrogel formations were created in order to demonstrate tunable mechanics. However, for each gel formulation it was desired to achieve a high and consistent electrical conductivity so that mechanical tuning did not overly impact electrical performance. Therefore, the highest amount of PEDOT was used that could be integrated into all gel formulations (0.26 wt\% PEDOT). Indeed, by altering AAc monomer and PEGDM crosslinker concentrations (AAc from 4 to $10 \mathrm{wt} \%$ and PEGDM from 1 to $5 \mathrm{wt} \%$ ), gel stiffness could be tuned over a range of moduli consistent with tissues (Figure A1). While different gel formulations yielded gels with different stiffnesses, the DC electrical properties of the gels were similar between gel formulations when subjected to voltages between 0.5 and $8 \mathrm{~V}$ (Figure A2). This indicates that the amount of acrylic acid and PEGDM crosslinker can be used to formulate gels with specific levels of stiffness without overly impacting gel electrical performance. Also, of note was that these gels were relatively tough and compressible, exhibiting particularly high strain of failures for soft, nanoporous gels (with some formulations surviving up to $88 \%$ compression before failing (Figure A3).

While potentially useful in many applications including neuroprosthetic, these nanoporous PEDOT/pAAc gels may still present several practical limitations for use in applications that demand implantation. For example, their introduction to tissues would require an invasive surgical procedure. Such procedures would be expensive, 
inconvenient, and could inflame the tissue (with downstream effects limiting electrical conductivity). An alternative approach would be to inject the gel precursor solution into the tissue and cast the gel in vivo. While the APS/TEMED chemistry used for gelation here could be inappropriate for this, other more biologically friendly gelation strategies could be employed. However, even then, an issue with casting a gel within or against neural tissues is that the gel will swell beyond its original size after injection (and many of these gel formulations indeed swell greatly beyond their original casting size, Figure A4). While the degrees of in vitro swelling observed here would likely not be as severe in vivo (where there is much less water available to facilitate swelling), even small degrees of swelling could be painful and potentially life-threatening in or against sensitive neural tissues. An alternative strategy would be to first cast the gels ex vivo, rinse residual gelation reagents from them, allow them to fully swell, cut them to desired shapes/sizes, and then inject them into the tissue of interest. An electrical lead would be introduced to the injected gel (through the void established by the injection needle) and the injected gel would serve as soft interfacial buffer between the hard lead and the soft tissue. This injection-based approach would require a conducting hydrogel with highly compressible mechanics. While the PEDOT gels presented here exhibited mechanical properties that would suggest a high degree of compressibility and potential injectability, they were not capable of surviving the strains associated with injection. For example, after swelling fully, a gel formulation that exhibited a combination of being soft with high conductivity and high strain of failure (the $9 \mathrm{wt} \%$ AAc, $0.26 \mathrm{wt} \%$ PEDOT, $1 \mathrm{wt} \%$ PEGDM gel) failed at $80 \%$ 
compression (Figure A5a) and did not survive after being passed through a 16-guage needle (Figure A5b).

In order to generate conductive hydrogel structures that were capable of surviving the stresses associated with injection after fully swelling, gels were engineered to have shape-memory properties [26] which can enable minimally invasive injection of hydrogel materials [27]. Shape-memory properties were achieved by adopting a cryogelation approach during fabrication [28] where a gel precursor solution was prepared (Figure 3.1a, i) and frozen during polymerization and crosslinking. Ice crystal nucleation and growth during freezing forced all gel constituents to the interstitial space between ice crystals, concentrating the matrix between ice crystals (Figure 3.1a, ii). After cryogelation, the mixture was thawed, leaving macropores in the spaces previously occupied by ice crystals (Figure 3.1a, iii). The result of this process was a three-dimensional structure entrapping PEDOT with a spongy, macroporous structure (Figure 3.1b). Just as with their room-temperature-cast counterparts, these PEDOT cryogels were capable of exhibiting a wide range of stiffness depending on their formulation (Figure 3.1c, i) with high strain of failures and toughness for soft hydrogels (Figure 3.1c, ii and iii). In fact, several gel formulations did not fail under $90 \%$ compression (Fig. 3.1c, ii and iii: denoted with $¥$ ). Thus, their actual strain of failure and toughness values could not be measured since compressions beyond 90\% were not attempted (in order to prevent equipment damage). Accordingly, many of the values presented here represent minimum strain of failure and toughness values. Elsewhere, similar cryogelation approaches have been employed to produce gels with interconnected macropores [29], moduli similar to 
biological tissues [30, 31], sufficient mechanical toughness to remain intact when exposed to cyclic compression (50\% compression, 1000 cycles) [32], and the ability to spring back to form after compression through needles [28]. The novelty here is that the PEDOT/pAAc cryogels also exhibited electrical attributes potentially useful in neuroprosthetic.
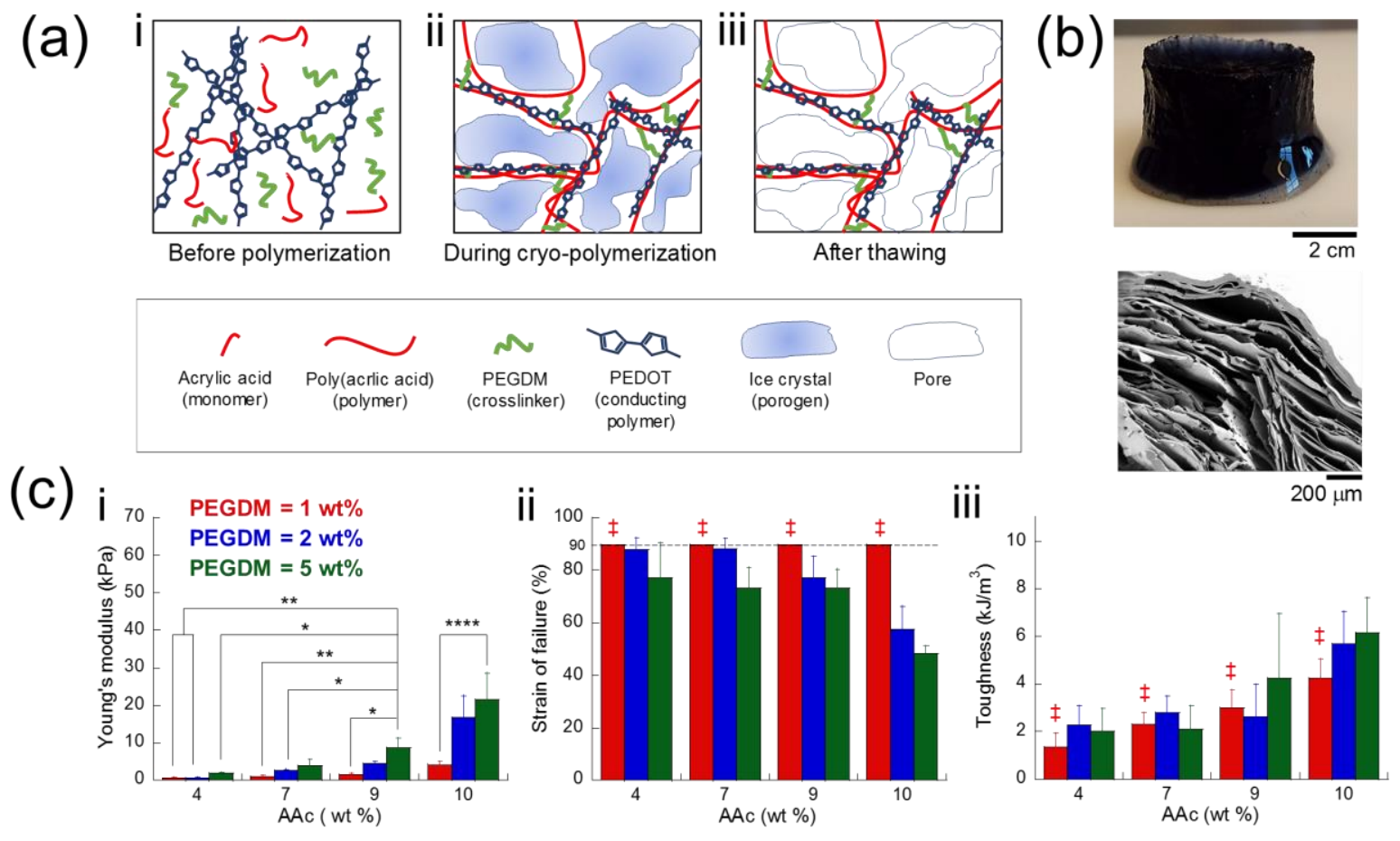

Figure 3.1. Cryogelation of PEDOT/pAAc hydrogels result in macroporous structures with soft, tunable, and compressible mechanics. (a) Schematic detailing the cryogelation process. (b) Photograph (top) and SEM cross-section (bottom) of a 9 wt\% AAc, 1 wt\% PEGDM, 0.26 wt\% PEDOT cryogel. (c) Compressive Young's modulus (i), strain of failure (ii), and toughness (iii) vs. acrylic acid concentration for PEDOT/pAAc cryogels that were crosslinked with 1 wt $\%$ (red), 2 wt $\%$ (blue), or 5 
wt $\%$ PEGDM. *, **, and **** indicate statistically significant differences with $p$ values of less than $0.05,0.01$, and 0.0001 , respectively $(\mathrm{N}=4) .+$ indicates that gels did not fail when compressed at $90 \%$ strain. Thus, strain of failure was at least $90 \%$ and hydrogel toughnesses were underestimates.

In order to explore the use of these hydrogels specifically in neuroprosthetic applications, PEDOT/pAAc cryogels were electrically characterized. Current vs. voltage curves (IV-curves) for a wide range of gel formulations showed, as expected, that conductivity was lower when excited at low voltages (Figure 3.2a, IV-curves had relatively small slopes at voltages between -2 and $+2 \mathrm{~V}$ ) and higher conductivities when excited at higher voltages (IV-curves had higher slopes at voltages less than and greater than -2 and $+2 \mathrm{~V}$, respectively). Computed conductivity values for various gel formulation and at various voltage excitation values can be found in Figure A6. While lower conductivities were recorded at voltage excitation levels lower than the overpotential at the metal-hydrogel interface, impedance measurements revealed that these cryogel systems (for gels cut to 2 x 12-mm cylinders after swelling using an LCR meter set to use a 2-volt signal) exhibited impedance magnitudes of $<200 \mathrm{Ohms}$ (Figure 3.2b) (for Nyquist plots of these same impedance data, see Figure A7 in Appendix A). Critically, because altering gel formulation can be used to tune gel mechanics (Figure 1c, i) without overly impacting gel conductivity (Figure 3.2a and $3.2 b$ ), cryogels could be customized to match the mechanics demanded by the application without sacrificing electrical performance. 
In order to determine if these electrical characteristics were sufficient to record low-powered neural signals, a specific cryogel formulation that exhibited both compressible mechanics (low Young's modulus and high strain-of-failure) and desirable electrical attributes (electrical conductivity nearing $1 \mathrm{~S} / \mathrm{cm}$ ) was selected for further study: the 9 wt $\%$ AAc, 0.26 wt \% PEDOT, 1 wt \% PEGDM cryogel. This cryogel formulation was cast on disc electrodes (Figure 3.2c, i: top), allowed to fully swell, and used to record signals emanating from a human subject's occipital lobe. These PEDOT cryogel-integrated disc electrodes (Figure 3.2c, i: bottom (blue)) were compared to electrodes integrated with Ten20 conducting paste (Figure 3.2c, i: second from top (red)) and a non-cryogelated PEDOT gel (second from bottom (green)). Compared to a popular EEG conducting paste (Ten20, which was found to be superior to other EEG conducting pastes on the market) [33]. electrodes with PEDOT cryogels were able to record neural signals at similar amplitudes and spectral powers (Figure 3.2c, ii \& iii: red and blue curves overlapped). When compared to a non-cryogelated PEDOT hydrogel (a formulation comparable to a PEDOT hydrogel reported elsewhere $[13,26,27,29-32,34-37]$ ), these cryogels also performed similarly (Figure 3.2c, ii \& iii: green and blue curves overlapped). Specifically, PEDOT cryogels provided similar degrees of signal and spectral resolution to those of a commercially available conductive paste and non-cryogel PEDOT gels. This enabled the observation of alpha waves when the subjects' eyes were closed (Figure 3.2c, ii, bottom: peak in signal from $7.5-12.5 \mathrm{~Hz}$ ) and decreased alpha waves when subjects opened their eyes (Figure 3.2c, ii \& iii, bottom: drop in signal power between $7.5-12.5 \mathrm{~Hz}$ with eyes 
opened (iii) vs. closed (ii)). Additionally, 1-hour exposure to PEDOT cryogels did not visually impact the skin (Figure A8).
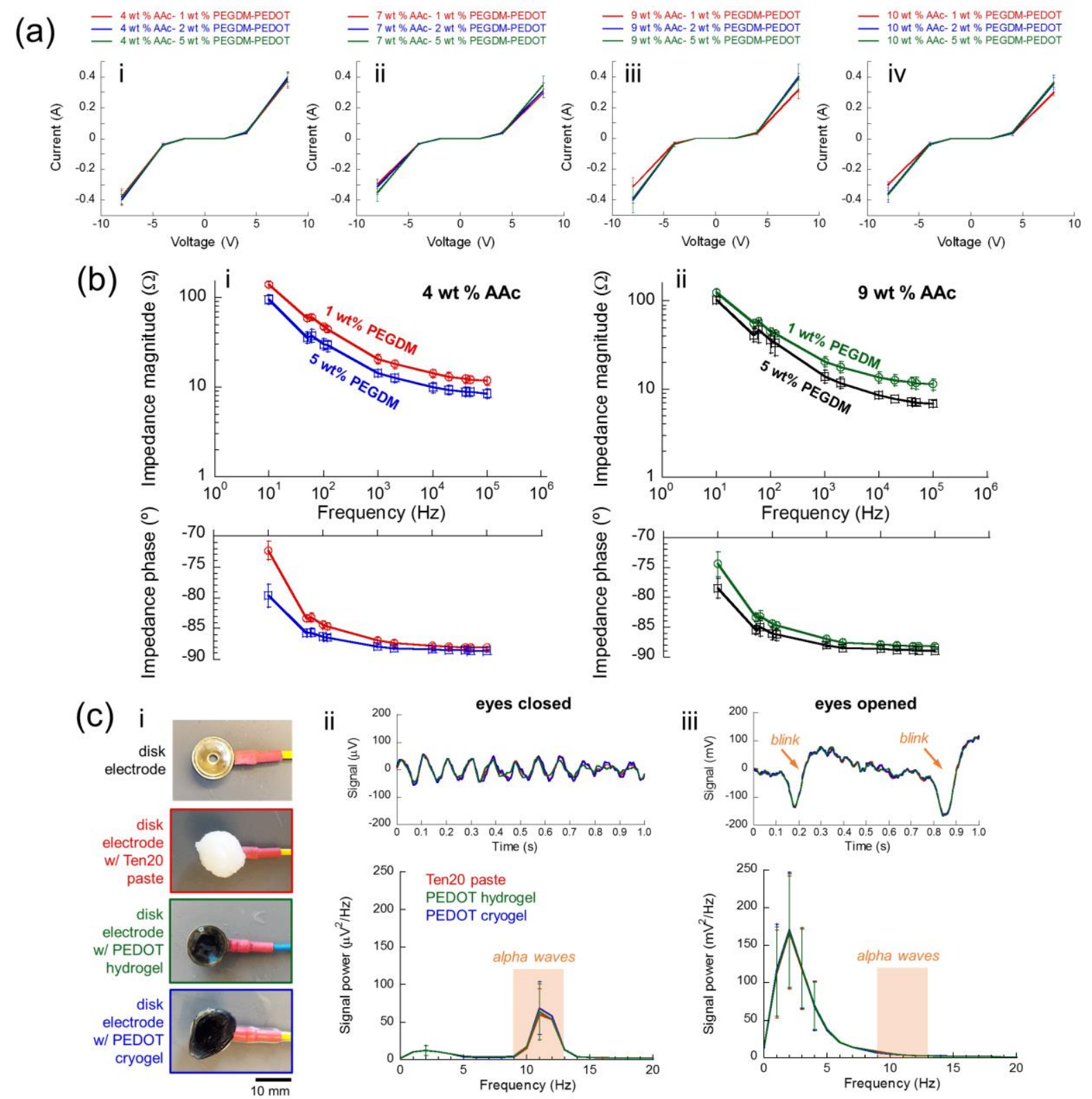

Figure 3.2. PEDOT/pAAc cryogels exhibited conductive electrical properties sufficient to record neural signals. (a) A schematic (i) and photo (ii) of a 9 wt\% AAc, 0.26 wt $\%$ PEDOT, $1 \mathrm{wt} \%$ PEGDM cryogel completing an LED circuit. (b) DC conductivity vs. acrylic acid concentration for PEDOT cryogels crosslinked using 1 
$\mathrm{wt} \%$ (red), $2 \mathrm{wt} \%$ (blue), and $5 \mathrm{wt} \%$ (blue) PEGDM. (c) (i) Impedance vs. frequency for $4 \mathrm{wt} \%$ AAc, $0.26 \mathrm{wt} \%$ PEDOT cryogels crosslinked with $1 \mathrm{wt} \%$ (red) and $5 \mathrm{wt} \%$ (blue) PEGDM. (ii) Impedance vs. frequency for $9 \mathrm{wt} \%$ AAc, $0.26 \mathrm{wt} \%$ PEDOT cryogels crosslinked with $1 \mathrm{wt} \%$ (red) and 5wt\% (blue) PEGDM. (d) (i) Images of disc electrodes with standard Ten20 paste (blue) and a PEDOT cryogel (red). (ii,iii) Recorded neural signal vs. time (top) and power spectrum (bottom) when subject's eyes are closed (ii) and open (iii). Insets: power spectrum from 7.5 to $12.5 \mathrm{~Hz}$, highlighting alpha wave power. For all parts, n.s. indicates that no statistical significance was found $(p>0.05)$ between any groups $(\mathrm{N}=4)$.

In order to examine how well a fully swollen cryogel retained its mechanical and electrical attributes after either treatment with ethanol (often used prior to placement on the skin) or autoclaving (for sterilization), $9 \mathrm{wt} \%$ AAc, $0.26 \mathrm{wt} \%$ PEDOT, 1 wt \% PEGDM cryogels were mechanically and electrically tested before and after ethanol-treatment and autoclaving. It was discovered that $70 \%$ ethanol treatment did not have a statistical impact on the cryogel's Young's modulus (Figure 3.3a, i: red and blue bars are statistically similar), suggesting that the cryogel's structure was not overtly altered by the treatment. However, autoclaving these cryogels was more disruptive and did have an impact on the gel's mechanical attributes (Figure 3.3a, comparing green and red bars/curves). While this may be a limitation for using these gels in vivo, note that they could potentially be produced as sterilized products in a clean environment with pre-sterilized materials. In terms of maintaining electrical properties before and after ethanol-treatment and autoclaving, 
DC conductivity was not statistically impacted by either ethanol-treatment or autoclaving when excited at $0.5 \mathrm{~V}$ (Figure 3.3a, ii: no statistically significant differences between red, blue, and green bars). This held true when excited at higher voltages as well (Figure A9).

Because these PEDOT cryogels exhibited particularly soft moduli and high strains of failure (Figure 3.1c) as well as the electrical ability to transmit neural signals (Figure 3.2c), experiments were conducted to examine if these cryogels could survive the compressions associated with injection. A cylindrical cryogel was compressed to $90 \%$ of its height and was able to eventually spring back to form after compression, remaining structurally intact (Figure 3.3b). This compressibility allowed these cryogels to survive being passed through a 16-gauge needle (Figure 3.3c). There have been other efforts to create injectable conducting hydrogels, though using in situ gelation to achieve injectability [38-40]. While adopting novel in vivo gelation strategies, these studies yielded gels that swelled after injection (from $120 \%$ to 500\%) which could be highly problematic since swelling could damage sensitive neural tissues. 
(a)

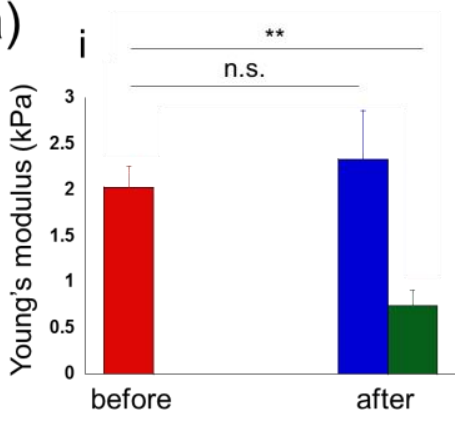

(b)

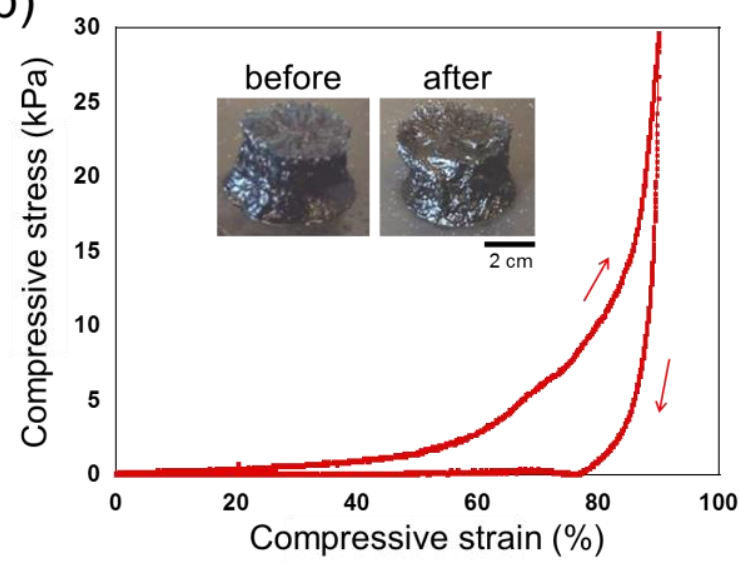

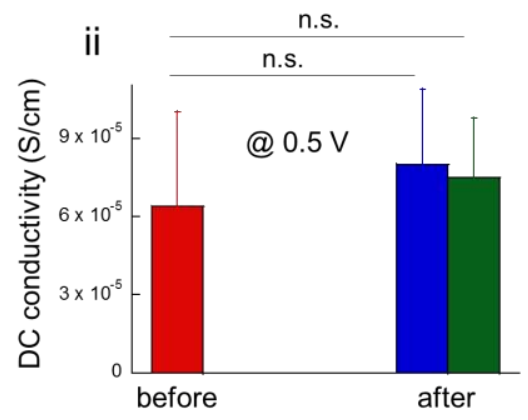

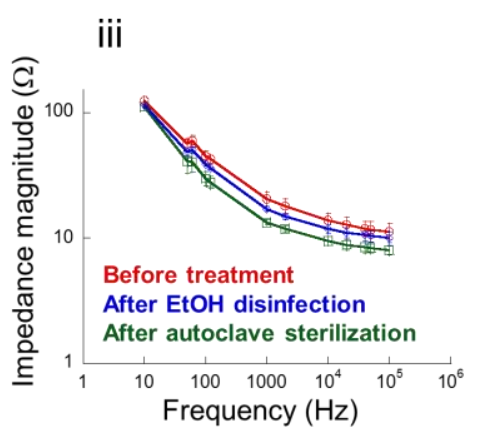

(c)

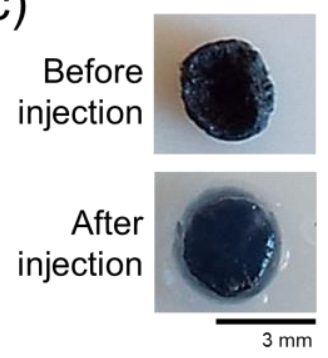

Hydrogel in syringe w/ 16gauge needle

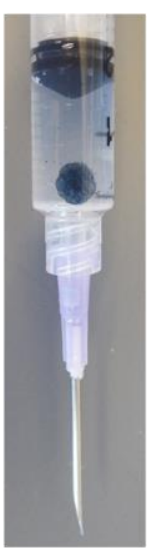

Figure 3.3. A PEDOT/pAAc cryogel can retain its mechanical and electrical attributes after being treated in ethanol and can survive high degrees of compression. (a) Young's modulus (i) DC conductivity when measured with a $0.5 \mathrm{~V}$ signal (ii), and impedance vs. frequency (iii) for $9 \mathrm{wt} \%$ AAc, $0.26 \mathrm{wt} \%$ PEDOT, 1 wt\% PEGDM cryogels before treatment (red) and after ethanol-treatment (blue) or autoclaving (red). (b) Stress vs. strain for the same cryogel when brought to and from 90\% compression. Inset: photographs of the cryogel before and after compression. (c) Photographs of a $3 \times 1.25 \mathrm{~mm}$ cylindrical cryogel (as formulated in parts (a) through (b)) before and after being passed through a 16-guage needle. 
In order to assess the viability of cells in the vicinity of these cryogels, fibroblasts studies were performed. 3T3 fibroblasts were plated and allowed to establish themselves for 24 hours. A 9 wt \% AAc, 0.26 wt \% PEDOT, 1 wt \% PEGDM cryogel was then placed directly on top of the $2 \mathrm{D}$ culture while fibroblast viability was monitored over the subsequent week and compared to controls (Figure 3.4a). LIVE/DEAD staining was performed (Figure 3.4b) to quantify cell population and viability over time. These analyses revealed that presence of a PEDOT cryogel did inhibited fibroblast growth vs time (Figure 3.4c, i: solid curve reaching lower cell densities than the dashed curve). This may have been the result of cells physically having less space to grow. In fact, the LIVE/DEAD image of the fibroblasts that grew for 7 days underneath a PEDOT cryogel reveals spots where cells did not invade (Figure 3.4b, bottom right fluorescent image). These could be locations where the cryogel made strong contact with the well-plate, physically limiting cell infiltration. The presence of a PEDOT cryogel, however, did not statistically impact fibroblast viability vs. time (Figure 3.4c, ii). Additionally, cell cycle analyses were performed on fibroblasts after exposure to PEDOT cryogels for 10 days (fibroblast doubling time is typically 18-24 hours). These studies revealed that PEDOT cryogel exposure did not statistically impact the progression of fibroblasts through their cell cycles (Figure 3.4c, iii). Propidium iodide (PI) histograms from these cell cycle analyses are provided in Supporting Information (Figure A10). The findings here are consistent with other reports. Elsewhere, PEDOT-based materials have (i) exhibited good biocompatibility with epithelial cells, neural cells, and fibroblasts [41], (ii) incited minimal inflammation in vivo $[42,43]$, and (iii) demonstrated the ability to reduce gliosis 
(glial-scar formation around materials implanted in brain tissue) [44]. However, further studies are needed to assess PEDOT cryogel biological performance. For example, viability, proliferation, and cell cycle analyses for other pertinent cells types (e.g., neurons and glia cells) will need to be performed. Additionally, in vivo studies will be needed to examine the performance of the specific PEDOT cryogel developed here, since the inflammatory response (which is not present in vitro) would play a critical role in implant performance.

Finally, a possible strategy for preserving an electrical pathway at the interface between neural tissues and electronic components in vivo is to have that interface locally deliver therapeutics that can help prevent fibrotic tissue formation. We hypothesized that these hydrogels would be particularly well-suited to locally deliver these types of therapeutics. To demonstrate this capability, 9 wt \% AAc, 0.26 wt \% PEDOT, 1 wt \% PEGDM cryogels were loaded with Interleukin-4 (IL-4) - a protein that can inhibit the inflammatory response of macrophages by differentiating them towards pro-repair phenotypes [45-47]. Release studies showed that IL-4 release was initially rapid, reaching $\sim 10 \mathrm{ng}$ of cumulative release within the first 24 hours, depending on the amount of protein loaded in the gel (Figure 3.4d, blue and red curves at 1 day). This initial burst release could be of particular use during the acute phase of inflammation immediately following cryogel introduction to the tissue. PEDOT cryogels continued to release IL-4 in lesser amounts over the course of the next 30 days (Figure 3.4d, blue and red curves after day 1). Additionally, these data showed that different amounts of cumulative IL-4 release could be achieved simply by loading 
the gels with different amounts of protein (Figure 3.4d, comparing the values of blue and red curves vs. time).

(a)

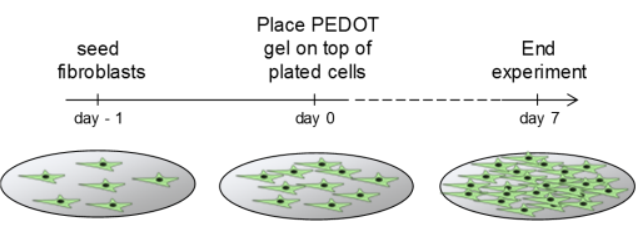

no gel
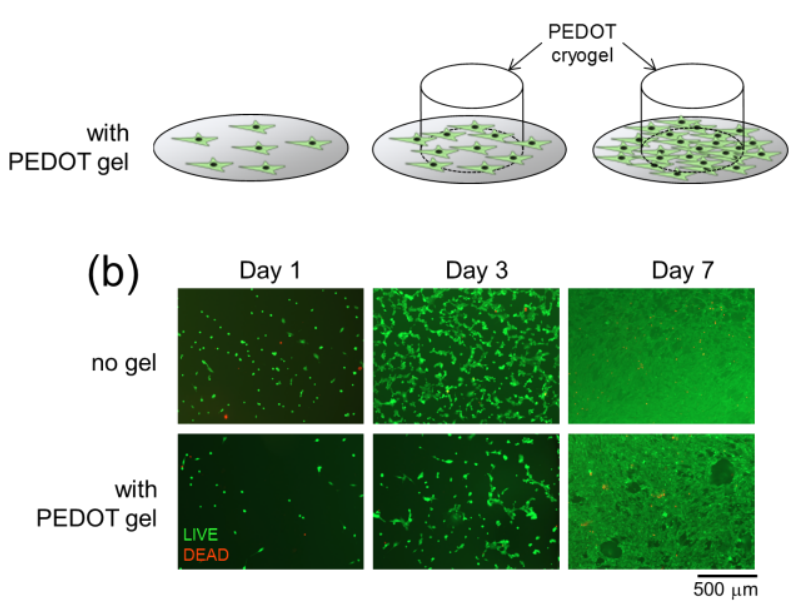

(d)

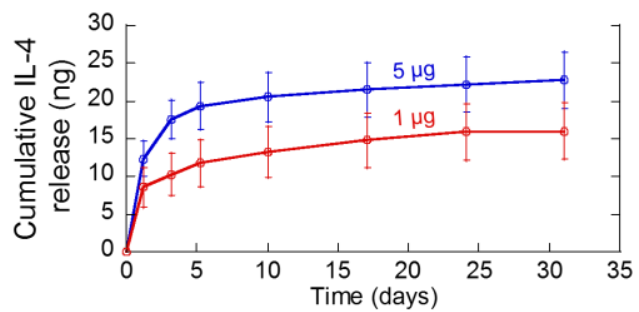

(c)
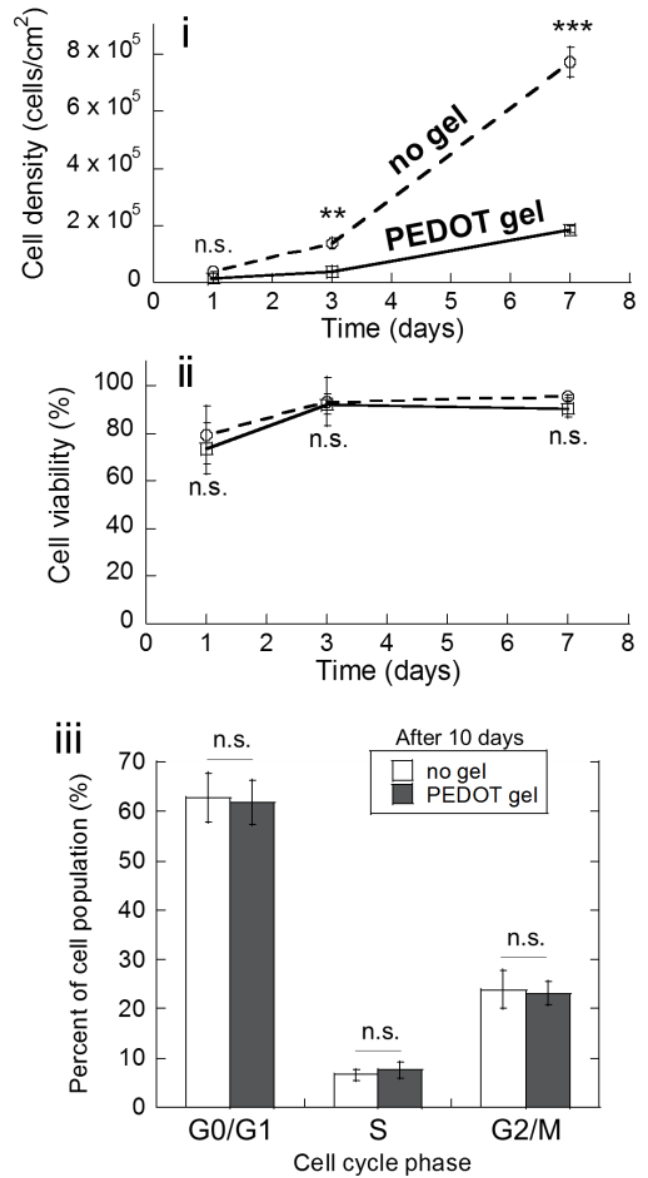

Figure 3.4. PEDOT cryogels do not disrupt fibroblast viability and cell cycle and can deliver proteins that may help reduce inflammation. (a) Schematic of in vitro viability experiments. (b) Green/red (live/dead) fluorescent images of 3T3 fibroblasts on days 1 through 7 when cells are not exposed to a gel (top) or exposed to a 9 wt\% AAc, 0.26 wt $\%$ PEDOT, 1 wt $\%$ PEGDM cryogel. (c) Cell density (i) and viability (ii) vs. time when cells are not exposed to a gel (dashed curve) or exposed to a the PEDOT cryogel (solid curve). (iii) Percent of fibroblasts in various stages of their cell 
cycle after 10 days of exposure to PEDOT cryogels (grey) vs. fibroblasts not exposed to gels (white). ${ }^{* *}$ and $* * *$ indicate statically differences between cryogel exposed cells and controls with $p<0.01$ and 0.001 , respectively. n.s. indicates that no statistical differences were found $(p>0.05) . \mathrm{N}=4$. (d) Cumulative IL-4 release vs. time for the PEDOT cryogel after being loaded with $1 \mu \mathrm{g}$ (red) or $5 \mu \mathrm{g}$ (blue) of IL-4. $\mathrm{N}=4$.

Despite not being injectable after fully swelling, the nanoporous PEDOT/pAAc room-temperature-cast hydrogels (characterized in Figs. A1-A5) exhibited attributes potentially beneficial in many neuroprosthetic applications. Their combination of soft but tunable mechanics (Figure A1: $0.5-65 \mathrm{kPa}$ ), toughness (Figure A3: $30-88 \%$ strain of failures, absorbing $1-7.5 \mathrm{~kJ} / \mathrm{m}^{3}$ before failing), ability to transmit neural signals (Figure 3.2c, green), and ability to remain hydrated (by virtue of being a crosslinked network of hydrophilic polymer) could be highly useful in applications where electrical contact must be maintained between electrical components and soft, curvilinear skin surfaces (e.g., EEG- and ECG-based applications). Use of these conductive hydrogels could provide improvements over the conductive pastes traditionally used for interfacing between electronics and tissues. For example, conductive hydrogels would involve less messy preparation, could conform better to irregularly surfaced skin, and could maintain electrical contact for longer periods of time without drying out.

The macroporous PEDOT/pAAc cryogels developed here (Figures 3.1 - 3.4) present several unique advantages for use in both topical and implanted 
neuroprosthetics applications. These conductive cryogels were composed of relatively inexpensive and biocompatible materials and were capable of being formed using straightforward techniques. Their tunable and soft $(0.2-22 \mathrm{kPa}$ Young's moduli) yet tough $\left(1.5-6 \mathrm{~kJ} / \mathrm{m}^{3}\right)$ and compressible $(50->90 \%$ strain of failures) mechanical properties provide specific advantages: (i) better mechanical matches with tissues than traditional metal electrodes alone and (ii) enable survivable compression through needles after fully swelling, and the ability to inject in or against sensitive tissues rather than surgical introduction. These desirable mechanics coincide with electrical properties that permit the recording of low-power neural signals, performing as well as conductive pastes that are currently commercially available (Figure 3.2c). Moreover, when compressed, these PEDOT cryogels' electrical resistances are significantly reduced (Figure A11, nearly 10-fold drop in DC resistance at $80 \%$ compressive strain), likely due to shortening of the electrical path. Thus, when pressed in or against tissues, they are expected to exhibit improved conductivity, enhancing their ability to record bio-electrical signals.

The mechanical and electrical attributes of the PEDOT/pAAc cryogels produced here build upon previous successes with formulating electrically conductive hydrogels. For example, hydrogels previously formed by interpenetrating electrically conductive polymer (such as polyaniline (PANi) or PEDOT) within crosslinked polymeric networks resulted in gels with conductivities on the order of 0.001 to 0.034 $\mathrm{S} / \mathrm{cm}$ and mechanics appropriate for many applications (fracture strains ranging from $60 \%$ to $90 \%$ and compressive stresses up to $1.1 \mathrm{MPa}$ ) [13, 15, 25, 35-38, 48]. One study [13] demonstrated that conducting PEDOT networks could be formed within 
dual networks (a poly(ethylene glycol) methyl ether methacrylate (PEGMA) network and a pAAc network), producing tough (surviving $80 \%$ compressive strains under $\sim 4$ MPa stresses with Young's moduli $\sim 90 \mathrm{kPa})$ and electrically conductive $(\sim 1 \mathrm{~S} / \mathrm{cm})$ gels at close to physiological $\mathrm{pHs}(\mathrm{pH}=6)$. Note that these dual network gels' mechanical and electrical properties could be further enhanced by subjecting them to acidic environments. While the dual network gels produced by Nacify et al. [13] provided excellent mechanical and electrical attributes for use as strain sensors, they likely lacked the highly compressible mechanics needed for post-swollen injection (i.e., a combination of very low Young's moduli and high strain of failures). Here, a simple cryogelation was used to produce conductive hydrogels with interconnected macroporous structures, thus endowing them with softer and compressible mechanics. This cryogelation strategy could likely be applied to other conducting hydrogels networks (such as those discussed above $[13,25,35-38,48]$ ) as a means to reduce stiffness and improve compressibility.

\subsection{Conclusion}

In summary, electrically conductive hydrogels with macroporous, soft, and compressible attributes were produced using a simple cryogelation approach and were shown to be capable of being passed through 16-guage needles for potential injection. The mechanical properties of these cryogels were tunable based on formulation, and alterations in cryogel formulation did not overly impact electrical performance. Cryogel electrical properties were sufficient to record low-power neural signals. These conductive cryogels did not impact fibroblast viability or cell cycle over the course of 
7 days but did hinder fibroblast growth and spreading compared to controls. Finally, these cryogels were capable of being loaded with anti-inflammatory protein (IL-4), releasing this protein over the course of a month. Taken altogether, these capabilities could improve interfaces between tissues and electronics in a wide range of neuroprosthetic applications and in other applications that demand soft but tough and tunable mechanical properties coupled with the ability to transmit electrical signals.

\section{Supporting Information}

Supporting Information is available at appendix A.

\section{Acknowledgements}

This work was supported by grant from the National Science Foundation (RII Track-2 FEC 1539068), a 3M Company Nontenured Faculty Award (32976949), and start-up support from the University of Rhode Island College of Engineering. The authors would like to thank Dr. Everett Crissman and the Rhode Island Consortium for Nanoscience \& Nanotechnology for their help with our SEM imaging. We would also like to thank Dr. Al Bach and Kim Andrews and the Rhode Island Idea Network for Biomedical Research Excellence's (RI-INBRE's) Centralized Research Core Facility (National Institutes of Health 2 P20 GM103430) for aiding in our fibroblast studies. 


\subsection{References}

[1] WHO, Neurological disorder: public health challenges, World Health Organization, Geneva, Switzerland, 2006.

[2] J. Leach, A.K. Achyuta, S. Murthy, Bridging the divide between neuroprosthetic design, tissue engineering and neurobiology, Frontiers in Neuroengineering 2(18) (2010).

[3] N.R. Williams, M.S. Okun, Deep brain stimulation (DBS) at the interface of neurology and psychiatry, J Clin Invest 123(11) (2013) 4546-56.

[4] M.K. Lyons, Deep brain stimulation: current and future clinical applications, Mayo Clin Proc 86(7) (2011) 662-72.

[5] M.L. Kringelbach, N. Jenkinson, S.L. Owen, T.Z. Aziz, Translational principles of deep brain stimulation, Nat Rev Neurosci 8(8) (2007) 623-35.

[6] R.T. Hassarati, W.F. Dueck, C. Tasche, P.M. Carter, L.A. Poole-Warren, R.A. Green, Improving cochlear implant properties through conductive hydrogel coatings, IEEE Trans Neural Syst Rehabil Eng 22(2) (2014) 411-8.

[7] R.A. Green, C.M. Williams, N.H. Lovell, L.A. Poole-Warren, Novel neural interface for implant electrodes: improving electroactivity of polypyrrole through MWNT incorporation, J Mater Sci Mater Med 19(4) (2008) 1625-9.

[8] P.J. Rousche, R.A. Normann, Chronic intracortical microstimulation (ICMS) of cat sensory cortex using the Utah intracortical electrode array, IEEE Transactions on Rehabilitation Engineering 7(1) (1999) 56-68. 
[9] P.J. Rousche, R.A. Normann, Chronic recording capability of the Utah Intracortical Electrode Array in cat sensory cortex, Journal of Neuroscience Methods 82(1) (1998) 1-15.

[10] L. Pan, G. Yu, D. Zhai, H.R. Lee, W. Zhao, N. Liu, H. Wang, B.C. Tee, Y. Shi, Y. Cui, Z. Bao, Hierarchical nanostructured conducting polymer hydrogel with high electrochemical activity, Proc Natl Acad Sci U S A 109(24) (2012) 9287-92.

[11] C.J. Kearney, D.J. Mooney, Macroscale delivery systems for molecular and cellular payloads, Nat Mater 12(11) (2013) 1004-17.

[12] K.C. Dee, D.A. Puleo, R. Bizios, An itroduction to tissue-biomaterial interactions, John Wiley \&Sons, Hoboken, 2003.

[13] S. Naficy, J.M. Razal, G.M. Spinks, G.G. Wallace, P.G. Whitten, Electrically Conductive, Tough Hydrogels with pH Sensitivity, Chemistry of Materials 24(17) (2012) 3425-3433.

[14] C. Uluturk, N. Alemdar, Electroconductive 3D polymeric network production by using polyaniline/chitosan-based hydrogel, Carbohydr Polym 193 (2018) 307315.

[15] M.R. Abidian, D.C. Martin, Multifunctional Nanobiomaterials for Neural Interfaces, Advanced Functional Materials 19(4) (2009) 573-585.

[16] P. Fattahi, G. Yang, G. Kim, M.R. Abidian, A review of organic and inorganic biomaterials for neural interfaces, Adv Mater 26(12) (2014) 1846-85.

[17] U.A. Aregueta-Robles, A.J. Woolley, L.A. Poole-Warren, N.H. Lovell, R.A. Green, Organic electrode coatings for next-generation neural interfaces, Frontiers in Neuroengineering 7(15) (2014). 
[18] J. Hur, K. Im, S.W. Kim, J. Kim, D.Y. Chung, T.H. Kim, K.H. Jo, J.H. Hahn, Z. Bao, S. Hwang, N. Park, Polypyrrole/Agarose-based electronically conductive and reversibly restorable hydrogel, ACS Nano 8(10) (2014) 10066-76.

[19] Y. Zhao, B. Liu, L. Pan, G. Yu, 3D nanostructured conductive polymer hydrogels for high-performance electrochemical devices, Energy \& Environmental Science 6(10) (2013).

[20] G.L. Mario Cheong, K.S. Lim, A. Jakubowicz, P.J. Martens, L.A. Poole-Warren, R.A. Green, Conductive hydrogels with tailored bioactivity for implantable electrode coatings, Acta Biomater 10(3) (2014) 1216-26.

[21] M.R. Abidian, D.H. Kim, D.C. Martin, Conducting-Polymer Nanotubes for Controlled Drug Release, Adv Mater 18(4) (2006) 405-409.

[22] M.R. Abidian, D.C. Martin, Experimental and theoretical characterization of implantable neural microelectrodes modified with conducting polymer nanotubes, Biomaterials 29(9) (2008) 1273-83.

[23] P. Huang, W. Chen, L. Yan, An inorganic-organic double network hydrogel of graphene and polymer, Nanoscale 5(13) (2013) 6034-9.

[24] S. Sekine, Y. Ido, T. Miyake, K. Nagamine, M. Nishizawa, Conducting polymer electrodes printed on hydrogel, J Am Chem Soc 132(38) (2010) 13174-5.

[25] W. Cho, J. Wu, B.S. Shim, W.F. Kuan, S.E. Mastroianni, W.S. Young, C.C. Kuo, T.H. Epps, 3rd, D.C. Martin, Synthesis and characterization of bicontinuous cubic poly(3,4-ethylene dioxythiophene) gyroid (PEDOT GYR) gels, Phys Chem Chem Phys 17(7) (2015) 5115-23. 
[26] F. Pilate, R. Mincheva, J. De Winter, P. Gerbaux, L. Wu, R. Todd, J.-M. Raquez, P. Dubois, Design of Multistimuli-Responsive Shape-Memory Polymer Materials by Reactive Extrusion, Chemistry of Materials 26(20) (2014) 5860-5867.

[27] L. Wang, J. Shansky, C. Borselli, D. Mooney, H. Vandenburgh, Design and fabrication of a biodegradable, covalently crosslinked shape-memory alginate scaffold for cell and growth factor delivery, Tissue Eng Part A 18(19-20) (2012) 2000-7.

[28] S.A. Bencherif, R.W. Sands, D. Bhatta, P. Arany, C.S. Verbeke, D.A. Edwards, D.J. Mooney, Injectable preformed scaffolds with shape-memory properties, Proc Natl Acad Sci U S A 109(48) (2012) 19590-5.

[29] A. Kumar, A. Srivastava, Cell separation using cryogel-based affinity chromatography, Nat Protoc 5(11) (2010) 1737-47.

[30] S. Kennedy, S. Bencherif, D. Norton, L. Weinstock, M. Mehta, D. Mooney, Rapid and extensive collapse from electrically responsive macroporous hydrogels, Adv Healthc Mater 3(4) (2014) 500-7.

[31] A.J. Engler, S. Sen, H.L. Sweeney, D.E. Discher, Matrix elasticity directs stem cell lineage specification, Cell 126(4) (2006) 677-89.

[32] C.A. Cezar, S.M. Kennedy, M. Mehta, J.C. Weaver, L. Gu, H. Vandenburgh, D.J. Mooney, Biphasic ferrogels for triggered drug and cell delivery, Adv Healthc Mater 3(11) (2014) 1869-76.

[33] P. Tallgren, S. Vanhatalo, K. Kaila, J. Voipio, Evaluation of commercially available electrodes and gels for recording of slow EEG potentials, Clin Neurophysiol 116(4) (2005) 799-806. 
[34] J.Y. Sun, X. Zhao, W.R. Illeperuma, O. Chaudhuri, K.H. Oh, D.J. Mooney, J.J. Vlassak, Z. Suo, Highly stretchable and tough hydrogels, Nature 489(7414) (2012) 133-6.

[35] T. Dai, X. Qing, Y. Lu, Y. Xia, Conducting hydrogels with enhanced mechanical strength, Polymer 50(22) (2009) 5236-5241.

[36] T. Dai, X. Qing, H. Zhou, C. Shen, J. Wang, Y. Lu, Mechanically strong conducting hydrogels with special double-network structure, Synthetic Metals 160(7-8) (2010) 791-796.

[37] T. Dai, Z. Shi, C. Shen, J. Wang, Y. Lu, Self-strengthened conducting polymer hydrogels, Synthetic Metals 160(9-10) (2010) 1101-1106.

[38] L. Li, J. Ge, P.X. Ma, B. Guo, Injectable conducting interpenetrating polymer network hydrogels from gelatin-graft-polyaniline and oxidized dextran with enhanced mechanical properties, RSC Advances 5(112) (2015) 92490-92498.

[39] L. Li, J. Ge, B. Guo, P.X. Ma, In situ forming biodegradable electroactive hydrogels, Polymer Chemistry 5(8) (2014).

[40] Y. Wu, B. Guo, P.X. Ma, Injectable Electroactive Hydrogels Formed via HostGuest Interactions, ACS Macro Letters 3(11) (2014) 1145-1150.

[41] R. Balint, N.J. Cassidy, S.H. Cartmell, Conductive polymers: towards a smart biomaterial for tissue engineering, Acta Biomater 10(6) (2014) 2341-53.

[42] L. Ghasemi-Mobarakeh, M.P. Prabhakaran, M. Morshed, M.H. Nasr-Esfahani, H. Baharvand, S. Kiani, S.S. Al-Deyab, S. Ramakrishna, Application of conductive polymers, scaffolds and electrical stimulation for nerve tissue engineering, $\mathrm{J}$ Tissue Eng Regen Med 5(4) (2011) e17-35. 
[43] S.C. Luo, E. Mohamed Ali, N.C. Tansil, H.H. Yu, S. Gao, E.A. Kantchev, J.Y. Ying, Poly(3,4-ethylenedioxythiophene) (PEDOT) nanobiointerfaces: thin, ultrasmooth, and functionalized PEDOT films with in vitro and in vivo biocompatibility, Langmuir 24(15) (2008) 8071-7.

[44] G. Cellot, P. Lagonegro, G. Tarabella, D. Scaini, F. Fabbri, S. Iannotta, M. Prato, G. Salviati, L. Ballerini, PEDOT:PSS Interfaces Support the Development of Neuronal Synaptic Networks with Reduced Neuroglia Response In vitro, Front Neurosci 9 (2015) 521.

[45] K.L. Spiller, R.R. Anfang, K.J. Spiller, J. Ng, K.R. Nakazawa, J.W. Daulton, G. Vunjak-Novakovic, The role of macrophage phenotype in vascularization of tissue engineering scaffolds, Biomaterials 35(15) (2014) 4477-88.

[46] K.L. Spiller, S. Nassiri, C.E. Witherel, R.R. Anfang, J. Ng, K.R. Nakazawa, T. Yu, G. Vunjak-Novakovic, Sequential delivery of immunomodulatory cytokines to facilitate the M1-to-M2 transition of macrophages and enhance vascularization of bone scaffolds, Biomaterials 37 (2015) 194-207.

[47] V.A. Kumar, N.L. Taylor, S. Shi, N.C. Wickremasinghe, R.N. D'Souza, J.D. Hartgerink, Self-assembling multidomain peptides tailor biological responses through biphasic release, Biomaterials 52 (2015) 71-8.

[48] M. Sasaki, B.C. Karikkineth, K. Nagamine, H. Kaji, K. Torimitsu, M. Nishizawa, Highly conductive stretchable and biocompatible electrode-hydrogel hybrids for advanced tissue engineering, Adv Healthc Mater 3(11) (2014) 1919-27. 


\section{CHAPTER 4}

\section{Integration of a Conductive Hydrogel Into a Tripolar Concentric Configuration to Improve Signal and Channel Isolation}

This manuscript is prepared to be submitted to

Advance Health Care Materials

By Rosa Ghatee ${ }^{1}$ Walter Besio ${ }^{2}$, Stephen Kennedy ${ }^{1,2}$

${ }^{1}$ Department of Chemical Engineering, ${ }^{2}$ Department of Electrical, Computer and Biomedical Engineering, University of Rhode Island, Kingston, Rhode Island 02881, USA 


\section{Abstract}

Electroencephalography (EEG) is one of the most important noninvasive methods for studying the brain's neural activity, and is used broadly in both diagnosis and research. However, conventional EEG methods do not provide adequate spatial resolution and signal-to-noise ratios for many emerging diagnostic and research applications. To address these inadequacies, high-resolution EEG methods are piquing the interest of researchers and clinicians. One such method, the use of tripolar concentric ring electrodes (TCREs), utilizes surface Laplacian theory and three concentrically arranged electrodes per sensor to address these issues. Although TCREs have been shown to significantly improve the spatial resolution and signal-to-noise of EEG signals it, like other electrode-to-scalp techniques, requires using conductive paste at the electrode-scalp interface. This not only electrically partially short-circuits the three concentric channels of the TCRE but also the paste can dry out over time. These issues prohibit TCREs from providing their true potential in terms of EEG spatial resolution and signal-to-noise ratio, especially in applications that require EEG recordings for prolonged periods of time. To address these issues, TCREs with electrically conductive hydrogels were developed to (i) eliminate the need for conductive paste, (ii) provide improved isolation between channels, and (iii) improve signal-to-noise ratio preservation over time. The aim of this work was to develop conductive hydrogels, integrate them into TCRE configurations, and characterize their sustained electrical properties over time as compared to TCREs with conductive paste. 
Key Words: conductive hydrogel, tripolar concentric ring electrode (TCRE), PEDOT, EEG

\subsection{Introduction}

Electroencephalography (EEG) is a technique used to measure electrical impulses generated by brain activity and is relatively affordable and easy to implement. The EEG provides excellent temporal resolution of complex brain signals which makes it ideal for diagnosing certain neurological diseases. For example, EEG is used to diagnose epilepsy, dementia, Alzheimer's disease [1], psychosis such as depression and schizophrenia [2,3], and narcolepsy [4]. In addition EEG can be used to evaluate the overall brain electrical activity of patients suffering from brain trauma [5, 6], extent of brain damage in comatose patients [7], drug intoxication, and encephalopathy [8]. Furthermore, EEG is extensively used in rehabilitation applications such as in brain-computer interfaces to provide control of prosthetic movement in patients with paralysis [9-12]. However, while EEG's excellent temporal resolution makes it highly desirable, EEG does not inherently provide good spatial resolution. This limits the use of EEG in emerging research and clinical theaters where excellent spatial and temporal resolutions are needed to monitor the spatiotemporally complex activities of the brain (e.g., real-time mapping/imaging of brain activity). If EEG techniques can be endowed with better spatial resolution, it will prove to be a powerful tool in a wide range of emerging and future neurological diagnostics and treatments. 
Because of this potential, there are several methods designed to improve the spatial resolution of EEG. Some of these methods, based on surface Laplacian theory, do not require predetermined modeling of the head's conductive heterogeneity, but nonetheless provide similar results as other methods that do require this predetermined modeling (e.g., cortical imaging) [13]. Surface Laplacian methods enhance spatial selectivity and source identification, attenuate universal noise, and improve signal-tonoise ratio. Moreover, surface Laplacian methods are independent of reference electrode location and even improve temporal resolutions [11, 14-17]. These attributes make these methods superb choices for high resolution EEG. There are many versions of surface Laplacian theory to process brain activity and different types of EEG electrode designs based on surface Laplacian theories. Most of these electrodes have a center conducting disc and one or more ring electrodes around the center disc; for example, a bipolar electrode, tripolar concentric ring electrode, and concentric ring electrodes (with one center disc and several rings around it). Increasing the number of rings around the center disc can enhance spatial selectivity even more, but improvements in spatial selectivity become negligible at some point. Studies have shown that electrodes with a center disc and two or three rings are the optimal $[18,19]$ (e.g., a center disc with two rings around it: a tripolar concentric ring electrode or TCRE, which will be investigated here) (Figure 4.1A).

Like traditional disc-shaped EEG electrodes, TCRE (and other multi-channel electrodes designed based on surface Laplacian theory) require electrical contact with the patient's scalp. To improve the electrical connection between an electrode and the patient's skin, a conductive paste is typically used [20]. While this paste significantly 
improves the electrode-skin interface connection, it also partially electrically shorts out the channels of TCRE configuration, somewhat limiting its ability to provide enhanced spatial selectivity (Figure 4.1B Right). To make matters worse, conductive pastes dry out over time, affecting signal quality and increasing noise over time. This limits their use in applications that involve EEG spatiotemporal analysis over prolonged periods of time (e.g., in wearable electronics). Thus, technologies that can provide prolonged conductive interfaces between TRCEs and a patient's scalp without shorting out the channels could enable a wide range of EEG-based therapies and diagnostics.

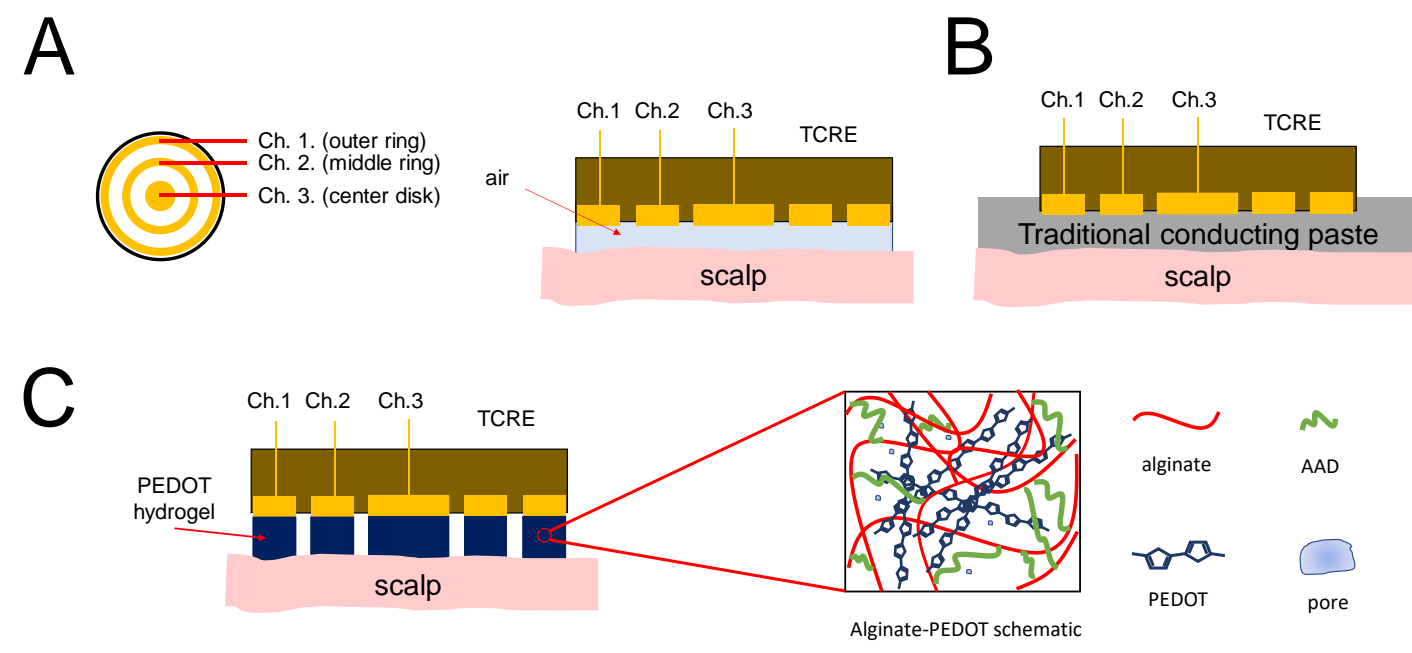

Figure 4.1. Conductive hydrogel not only provides contact between electrode and skin but also improves isolation between channels. Comparison of electrode's contact with skin with different conductive interfaces. (A) Tripolar concentric ring electrode (TCRE). (B) Left, TCRE in contact with skin without any interface, Right, TCRE with conductive paste (TEN20 paste) in contact with skin. (C) Tripolar 
concentric ring hydrogel electrode (TCRHE) in contact with skin on left and schematic of conductive hydrogel on right.

Hydrogels are soft materials which are made from cross-linked hydrophilic polymer networks [21]. Although they are insoluble in water, they can preserve a large fraction of water within their network for prolonged periods of time and retain 3D shapes, unlike conductive pastes [22]. However, hydrogels typically exhibit poor conductivity [23]. Although this is not an issue in many applications, for use as an interface between electrodes and skin, conductivity is a critical priority [24, 25]. Conductive polymer hydrogels are a unique class of hydrogels which not only have soft mechanical properties similar to skin [26], but also have electrical properties like metals and inorganic semiconductors [27]. There are several conductive polymers available which are widely used for fabricating conductive hydrogels, though for use as electrodes in neural interfaces: polypyrrole and poly(3-4, ethylenedioxythiophene) (PEDOT) are primarily used [28]. It has recently been demonstrated that PEDOT provides improved conductivity and stability in oxidized environments over polypyrrole [22, 24, 29-32]. Moreover, PEDOT is biocompatible [33]. Thus, PEDOT will be employed as the conductive component of the hydrogel electrodes.

While PEDOT hydrogels have been created previously, they have yet to be employed as a means to enhance spatial resolution of multi-electrode systems by providing soft, 3D conducting structures that retain their shape and moisture over prolonged periods of time. Thus, the work proposed here is a novel step forward in the context of contemporary technologies. The hypothesis guiding this work is that electrodes can be made from conductive hydrogels which can be formed in any 
desirable shape (i.e., a TCRE configuration) and retain that shape, thus providing improved isolation between TCRE channels and prolonged conductivity and contact with scalp (Figure 4.1C). Specifically, this study involves the synthesis of conductive hydrogels with multiple polymers and crosslinkers and evaluation of their structural, mechanical and electrical behaviors to identify an optimal hydrogel formulation to integrate into TCRE configuration and refine electrode design to optimize its performance and improve channel isolation. Moreover, we performed EEG testing to compare hydrogel-integrated TCRE (TCRHE) to regular TCRE with conductive paste.

\subsection{Materials and Methods}

\subsubsection{Materials}

For alginate-PEDOT (alg-PEDOT) hydrogels, alginate (PROTANAL LFR5/60) was purchased from FMC. Adipic acid dihydrazide (AAD), 1hydroxybenzotriazole hydrate (HOBt), N-(3-dimethylaminopropyl)-N'ethylcarbodiimide hydrochloride (EDC), poly(3,4-ethylenedioxythiophene)poly(styrene sulfonate) (PEDOT:PSS) and phosphate buffered saline (PBS) were purchased from Sigma-Aldrich (St. Louis, MS). Sigmacote for coating glass sheets was purchased from Sigma-Aldrich (St. Louis, MS). All materials were used without any modification.

For polyacrylic acid-PEDOT (PAAc-PEDOT) hydrogels, acrylic acid (AAc), sodium hydroxide $(\mathrm{NaOH})$, poly(ethylene glycol) dimethacrylate (PEGDM), poly(3,4ethylenedioxythiophene):polystyrene sulfonate (PEDOT:PSS or PEDOT from now 
on), N,N,N',N'-Tetramethylethylenediamine (TEMED), ammonium persulfate (APS), aluminum oxide column, and phosphate buffered saline (PBS) were purchased from Sigma Aldrich. All materials were used without any modification, except AAc, which was purified using aluminum oxide column to remove the inhibitor.

To fabricate the tripolar concentric ring hydrogel electrode (TCRHE), a tripolar concentric ring electrodes (TCREs) were acquired from CREmedical. The tripolar concentric ring mold (TCR mold) was printed using a 3D printer. Epoxy (marine epoxy) and sealant were purchased from Amazon and the conductive hydrogel was fabricated in a manner described in detail in the next sections.

\subsubsection{Hydrogel synthesis}

For preparation of alg-PEDOT hydrogels, alg-AAD solution was prepared first, where different amounts of alginate $(2-5 \mathrm{wt} \%)$ were added to deionized water (DI water) and stirred until fully dissolved. The AAD (2.5 and $5 \mathrm{mM}$ ) and HOBt were added to the mixture until fully dissolved, and the resulting solutions were stored at $4^{\circ} \mathrm{C} .2 \mathrm{ml}$ of alg-AAD solution was mixed with $2.5 \mathrm{ml}$ of PEDOT, $0.5 \mathrm{ml}$ of EDC (50 $\mathrm{mg} / \mathrm{ml}$ ) was added, and immediately after mixing the mixture was poured onto a Sigmacoted glass sheet. Another piece of Sigmacoted glass sheet was placed on top of the solution with $2 \mathrm{~mm}$ spacing, and after 30 minutes hydrogels were cut with a biopsy punch and stored in PBS.

To make pAAc-PEDOT hydrogels, different amounts of purified AAc (4 -10 wt \%), PEGDM solution (1, 2 and 5 wt \%), $\mathrm{NaOH}$ solution (465 $\mu \mathrm{l}$ per ml of AAc), PEDOT (0.26 wt \%) and DI water (depending on hydrogel formulation DI water may 
or may not be added) were added and vortexed. TEMED and APS (137 mg/ml) were then added to the solution to initiate gelation. This solution was immediately transferred to $10 \times 10 \mathrm{~mm}$ cylindrical Teflon molds and left to gel at room temperature for three hours. Samples were then removed and stored in PBS.

\subsubsection{Degree of swelling}

To measure degree of swelling, the height and diameters of the hydrogels were measure before immersion into PBS to evaluate their volume $\left(\mathrm{V}_{\text {before }}\right)$. After samples were fully swollen and had reached equilibrium their height and diameters were measured again $\left(\mathrm{V}_{\mathrm{after}}\right)$, and degree of swelling was calculated from:

$$
\text { degree of swelling }=\frac{V_{\text {after }}}{V_{\text {before }}} \quad \text { Eq. } 4.1
$$

\subsubsection{Mechanical characterization}

The Young's modulus, strain of failure $(\mathrm{SoF})$ and toughness of the hydrogels were quantitively measured using a compression testing. For mechanical characterization, the sample was placed between grids of an Instron Universal Testing System (Model 3345) and compressed until failure. After the compression test on a given sample, a stress-strain curve was obtained, and mechanical properties such as Young's modulus, strain of failure and toughness were obtained from this curve.

\subsubsection{Electrical characterization}


Prior to measuring the electrical properties of the hydrogels, fully swollen samples were cut into discs $12 \pm 0.1 \mathrm{~mm}$ in diameter and $2 \pm 0.1 \mathrm{~mm}$ in height. A sample was then placed between electrodes and -8, -2, -1.5, 1.5, 2 and 8 Volts (Electro industries DIGI 360) were applied, and the current (Fluke 115) was measured. For measuring impedance (LCR-6300), hydrogels were exposed to different frequencies $(10,50,60,100,120,1000,2000,10000,20000,40000,50000$ and $100000 \mathrm{~Hz}$ at 10 $\mathrm{mv}$ ) and the magnitude of impedance and phase angle were recorded.

\subsubsection{Hydrogel electrode fabrication}

Molds with ring sizes and heights similar to those of the TCRE were printed using a 3D printer (Original Prusa i3 MK2). Figure 4.2A show the TCRE, and Figure 4.2B illustrates the mold design. Sealant was applied on the edge of the electrode wall between the rings (Figures 4.2C (i) and 4.2D (i)) to improve insulation between the rings. A traditional TCRE (prepared ahead of time) was inserted inside the mold and the electrode back was covered with epoxy. Hydrogels were prepared as described previously, and were injected into the mold, where Figures $4.2 \mathrm{C}$ (iv) and 4.2D (iv) show the prepared TCRHE. 
A

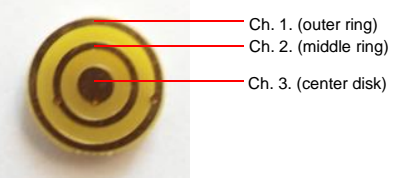

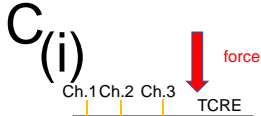

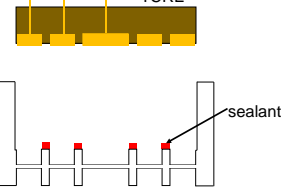

TCR hydrogel mold

D(i)

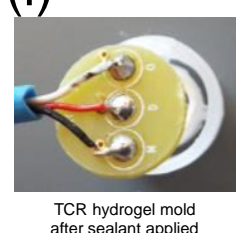

$\mathrm{B}$
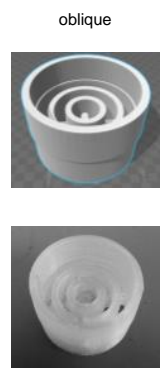

(ii)

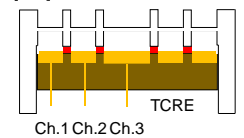
TCR hydrogel mold with
electrode snapped into place

(ii)

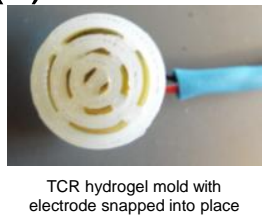

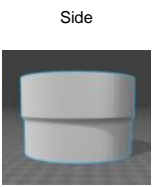

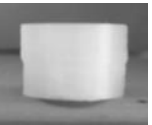

(iii)

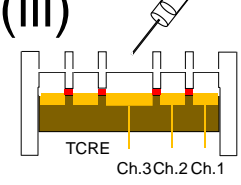

Injecting hydrogel mixture into

(iii)

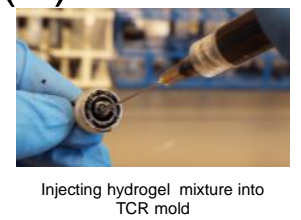

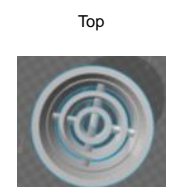

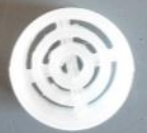

(iv)

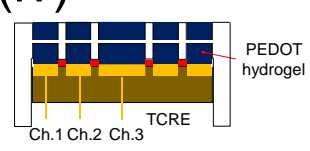

TCR hydrogel electrode

(iv)

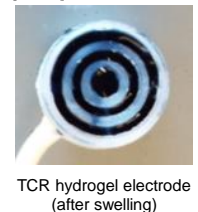

Figure 4.2. Fabrication of tripolar concentric ring hydrogel electrode (TCRHE).

(A) Tripolar concentric ring electrode (TCRE). (B) TCR mold holds both the TCRE and conductive hydrogel. (i) TCR mold oblique view. (ii) TCR mold side view, (iii) TCR mold top view. (C) Schematic of fabricating TCRHE step-by-step. (i) Applying sealant on the edge of wall between rings to isolate channels and inserting TCRE. (ii) TCRE is inserted into mold and mold is ready for injection of hydrogel. (iii) Hydrogel is being injected with 22 gauge needle. (iv) TCRHE. (D) Fabrication of TCRHE step by step. (i) Sealant is applied on the edge of walls between rings to isolate channels and inserting TCRE. (ii) TCR mold is ready for injection of hydrogel. (iii) hydrogel is being injected with 22 gauge needle into TCR mold. (iv) TCRHE. Hydrogel mixture shown here is $2 \mathrm{w} \%$ alginate, $2.5 \mathrm{mM}$ AAD and $0.26 \mathrm{wt} \%$ PEDOT. 


\subsubsection{Insulation between rings}

To measure the insulation between the channels, impedance between the channels was measured using an LCR meter. Wires attached to each ring were connected to the impedance meter, and impedance was measured at 10,50,60, 100, 120 and $1000 \mathrm{~Hz}$ with a $10 \mathrm{mv}$ excitation voltage in ideal conditions, where TCRHE and TCRE with Ten20 paste were exposed to air.

\subsubsection{Electroencephalography (EEG) testing}

EEG test was performed on four volunteers, two male and two females. All volunteers signed IRB consent form. A typical EEG test setup was used. Briefly, ground and reference electrodes (gold disc electrodes with Ten20 paste) were put on the forehead of a patient, and TCRHEs and TCRE were placed on the occipital lobe using a headband. A TCRE with Ten20 paste (as the conductive interface) was in the center $(\mathrm{Oz})$ and TCRHEs were on the left $(\mathrm{O} 1)$ and right $(\mathrm{O} 2)$ occipital lobes. The electrode attachment sites were swiped with Nuprep prior to testing. The volunteer was asked to alternately open and close their eyes for about 60 seconds periods. The tEEG and TCRHE signals were pre-amplified (187.5 V/V) with a t-Interface 202.0 (CREmedical Corp.), then amplified (1000 V/V) and digitized (200 S/s) with a Grass

Comet Plus (Grass technologies West Warwick RI) by notch filtering with range of 1$70 \mathrm{~Hz}$ while recording. I assume you used a tInterface.

\subsubsection{EEG longevity testing}


A volunteer was asked to wear electrodes for $4 \mathrm{~h}$ and EEG testing was performed using the method previously described. The volunteer moved around freely while wearing the electrodes.

\subsubsection{Signal-to-noise ratio}

The noise was estimated by obtaining the power spectrum between blinks ( $1 \mathrm{~s})$ and the signal-to-noise ratio was derived by dividing the power spectrum for the condition with eyes closed by the noise.

\subsubsection{Statistical analysis}

All experiments were performed at least four times. The results were reported as mean \pm standard deviation. MATLAB was used to evaluate FFT on EEG results to acquire the power spectra and signal-to-noise ratios. Statistical analysis was performed using ANOVA (analysis of variance) with Tukey's HSD (honestly significant difference) test at a significance level of 0.05 . * indicates p-values $<0.05, * *$ indicates

p-values $<0.01, * * *$ indicates p-values $<0.001$ and $* * * *$ indicates p-values $<0.0001$.

\subsection{Results and discussion}

\subsubsection{Hydrogel synthesis and degree of swelling}

To successfully fabricate a tripolar concentric ring hydrogel electrode, a conductive hydrogel formulation that can remain intact inside the mold should first be identified. To meet this criterion, PAAc-PEDOT hydrogels and alg-PEDOT hydrogels 
with different polymer and cross-linker concentrations were fabricated. The conductive polymer (PEDOT) concentration remained constant $(0.26$ wt $\%)$ for all hydrogel formulations. $0.26 \mathrm{wt} \%$ PEDOT was the maximum concentration needed since initial experiments showed that using higher concentrations of PEDOT did not improve gel conductivity (data not shown). As shown in Figure 4.3, hydrogel volumes were measures before and after being fully swollen in PBS to measure and compare the degree of swelling, which was between $3-12$ for PAAc-PEDOT hydrogels (Figure 3A(iii)). PAAc-PEDOT hydrogels were injected into a TCR configuration, and as expected, they swelled significantly and did not remain inside the mold. Figures 4.3A (i) and 4.3A (ii) show two different PAAc formulations with the lowest and highest degrees of swelling, respectively.

On the other hand, the degree of swelling remained around 1 for the algPEDOT formulations (Figure 4.3B (ii)), indicating that the degree of swelling for algPEDOT hydrogels was independent of their formulation parameters. Alg-PEDOT hydrogel formulations were also injected into a TCR mold, and remained in the mold, proving that alg-PEDOT hydrogels had a degree of swelling around 1 (Figure 4.3B (i)). The significant differences in the swelling behavior of PAAc-PEDOT and algPEDOT hydrogels are due to the differences in the polymer chains of alginate and polyacrylic acid. In the hydrogel formulations, sodium ions are present to make crosslinking possible. When the hydrogel swells in PBS, there is a $\mathrm{pH}$ difference between hydrogel network and surrounding medium, and water moves into the hydrogel matrix until it reaches equilibrium. There are more sodium ions within the PAAc hydrogel network than the alginate hydrogel network, which causes PAAc hydrogels to swell 
more than alginate hydrogels. Figure $4.3 \mathrm{~A}$ (iii) demonstrates that by increasing the amount of polymer present in the formulation that the degree of swelling increases, since with increasing polymer concentration, sodium ion concentration increases for both alginate and PAAc. However, there is a higher concentration of sodium oxide within PAAc-PEDOT hydrogel formulations in comparison to alg-PEDOT hydrogels.

A
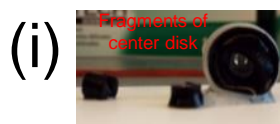

(ii)

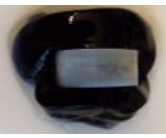

(iii)

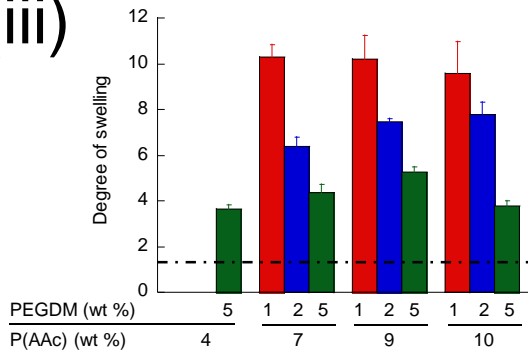

B

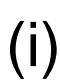
2 wt\% alginate- 2.5 mM AAD-PEDOT

(ii)

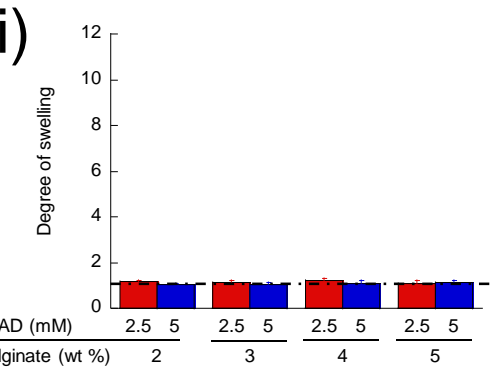

Figure 4.3. Alg-PEDOT hydrogel exhibit comparably little swelling. Comparison of the degree of swelling for different conductive hydrogels with different polymers including (A) $\mathrm{P}(\mathrm{AAc})$ and (B) alginate as the backbone. (A) Integration of $\mathrm{P}(\mathrm{AAc})-$ PEDOT hydrogel into TCR format for (i) hydrogel with 4 wt \% P(AAc), 5 wt \% PEGDM and 0.26 wt \% PEDOT (lowest degree of swelling). (ii) Hydrogel with 9 wt $\%$ P(AAc), 1 wt \% PEGDM and 0.26 wt \% PEDOT. (iii) Comparison of degree swelling for a wide range of $\mathrm{P}(\mathrm{AAc})(4-10 \mathrm{wt} \%)$ and PEGDM (1, 2 and $5 \mathrm{wt} \%)$ with constant 0.26 wt \% PEDOT. (B) Hydrogel made from different concentration of alginate and AAD have a swelling degree around 1. (i) Alginate-PEDOT hydrogel integrated into TCR format for hydrogel with $2 \mathrm{wt} \%$ alginate, $2.5 \mathrm{mM}$ and $0.26 \mathrm{wt} \%$ 
PEDOT. (ii) Comparison of degree of swelling for a wide range of alginate (2 - $5 \mathrm{wt}$ $\%)$ and $\operatorname{AAD}(2.5 \& 5 \mathrm{mM})$ with constant $0.26 \mathrm{wt} \%$ PEDOT. $\mathrm{N}=4$ for all parts.

\subsubsection{Mechanical properties}

For diagnostic purposes, patients frequently are asked to wear electrodes for up to 24 hours [34]. To make testing more bearable for patients, especially children, we aimed to use conductive hydrogels that have similar mechanical properties to skin, and at the same time ones that exhibit favorable toughness to ensure long-term integrity of the electrodes. To do so, the mechanical properties of the hydrogels, namely Young's modulus, strain of failure and toughness were measured (Figure 4.4). Figure 4.4A shows the mechanical properties of alg-PEDOT hydrogel formulations. The Young's modulus was between $2-16 \mathrm{kPa}$ for these formulations, and these data indicated that with increasing polymer and crosslinking concentrations, the Young's modulus increases. The alg-PEDOT formulations had Young's modulus values similar to skin (Young's modulus between $50-150 \mathrm{kPa}$ [35]). Furthermore, these hydrogel formulations demonstrated toughness between $2-25 \mathrm{~kJ} / \mathrm{m}^{3}$ (Figure 4.4B), where the toughness increased with increasing alginate and cross-linker concentrations. The hydrogel formulations can withstand high strains (75 \% - 83\%) (Figure 4.4C) and algPEDOT hydrogels with 2 wt $\%$ alginate $/ 2.5 \mathrm{mM} \mathrm{AAD/0.26} \mathrm{wt} \mathrm{\%} \mathrm{PEDOT} \mathrm{had} \mathrm{a}$ significantly higher strain of failure in comparison to other formulations. Toughness and strain of failure are important properties to ensure the long-term robustness, and consequently, the integrity of electrodes. 
There have been reports of fabricating conductive hydrogels with PEDOT [36] and 3-4, ethylenedioxythiophene (EDOT) monomer (monomer form of PEDOT) [22]. In these studies, other synthesis methods were used to fabricate a double network conductive hydrogel which resulted in high Young's modulus and toughness. Critically, in our study, we demonstrated the ability to explicitly tune hydrogel mechanical properties by varying the polymer and cross-linker concentrations. Moreover, the method of conductive hydrogel synthesis described here enables a relatively fast and straightforward gel fabrication method, and can be adapted for a variety of polymers to achieve desirable mechanical properties.
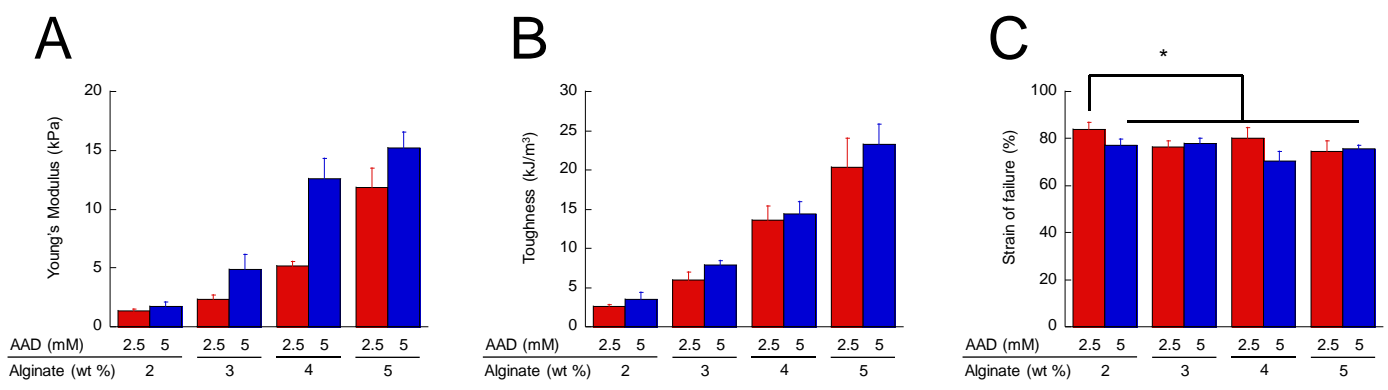

Figure 4.4. Hydrogel mechanics are tunable while degree of swelling remains around 1. Different hydrogel formulations made from alginate (2- $5 \mathrm{wt} \%$ ) and crosslinked with AAD $(2.5 \& 5 \mathrm{mM})$ with constant $0.26 \mathrm{wt} \%$ PEDOT. Mechanical characteristics of hydrogels including (A) Young's modulus, (B) toughness, and (C) strain of failure. $\mathrm{N}=4$ for all studies.

\subsubsection{Electrical properties}

Hydrogel electrical properties are independent of their formulation as shown in Figure 4.5. Figure 4.5A shows the IV curves for alg-PEDOT formulations with 
different alginate and crosslinker concentrations at different voltages. At each measured voltage, there were no statistically significant difference in the current read from hydrogel formulation. Also, the impedance of the hydrogels was measured at different frequencies at $10 \mathrm{mv}$, and the impedance of the hydrogels decreased with increasing frequency for all gel formulations. These data confirmed that there was no statistically significant difference in impedance of the hydrogel formulations at each frequency (Figure 4.5B). Moreover, the conductivity of these hydrogels was evaluated at $1.5 \mathrm{v}, 2 \mathrm{v}$ and $8 \mathrm{v}$ to have a better understanding of their behavior at given voltages (Figure 4.5C), where the conductivity of hydrogels increased with increasing voltage. The highest conductivity (about $1 \mathrm{~S} / \mathrm{cm}$ ) was achieved at $\pm 8 \mathrm{v}$.

The conductivity of the hydrogels at any given voltage is controlled by overpotential. The independence of electrical properties from the formulations was expected since the same amount of PEDOT $(0.26 \mathrm{wt} \%)$ was used for all formulations. Furthermore, our early tests showed that the electrical properties of the hydrogels did not change with changing PEDOT formulation (data not shown). Independence of electrical behavior from the formulation is beneficial as it allows for the hydrogel properties to be tuned depending on the application requirements. A variety of conductive hydrogel formulations with different amounts of polymer and conductive polymer components have been designed, resulting in systems with different properties. For example, depending on the synthesis method and PEDOT/EDOT concentrations, conductivities between $6.8 \times 10^{-4}$ to $1 \mathrm{~S} / \mathrm{cm}$ have been reported for conductive hydrogels [22, 33, 37-40]. Zhao et al. [36] reported conductivities greater than $3.3 \mathrm{~S} / \mathrm{cm}$ for conductive hydrogels comprised of both PEDOT and polypyrrole 
(PPy) conductive polymers. The electrical conductivities reported here and in the described studies [22, 33, 37-40] are likely appropriate for electrical stimulation and sensing of neural tissue. For instance, the conductivities of scalp, skull and brain tissue are $0.33 \times 10^{-3}, 0.0042 \times 10^{-3}$ and $0.33 \times 10^{-3} \mathrm{~S} / \mathrm{cm}$, respectively [41, 42]. Overall, electrode materials should exhibit high conductivities for proper sensing and stimulation, and the hydrogels designed in this study provide electrical conductivities well above the described tissues. Furthermore, since the hydrogel formulations exhibited similar electrical properties, alg-PEDOT hydrogels with 2 wt \% alginate, 2.5 mM AAD and 0.26 wt \% PEDOT were chosen for fabricating TCRHE since they had the highest strain of failure. 
C

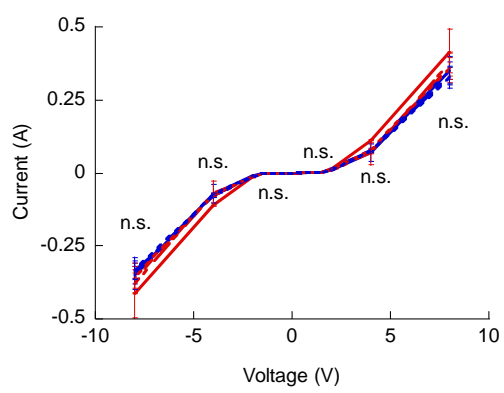

- 2 wt \% alg-2.5 mM AAD-PEDOT $2 \mathrm{wt} \%$ alg-5 mM AAD-PEDOT -. 3 wt \% alg- 2.5 mM AAD-PEDOT

- - 3 wt \% alg- 5 mM AAD-PEDOT

- - $4 \mathrm{wt} \%$ alg- 2.5 mM AAD-PEDOT

- - 4 wt $\%$ alg- 5 mM AAD-PEDOT

---- 5 wt \% alg-2.5 mM AAD-PEDO

B 5 wt \% alg-5 mMAAD-PEDOT

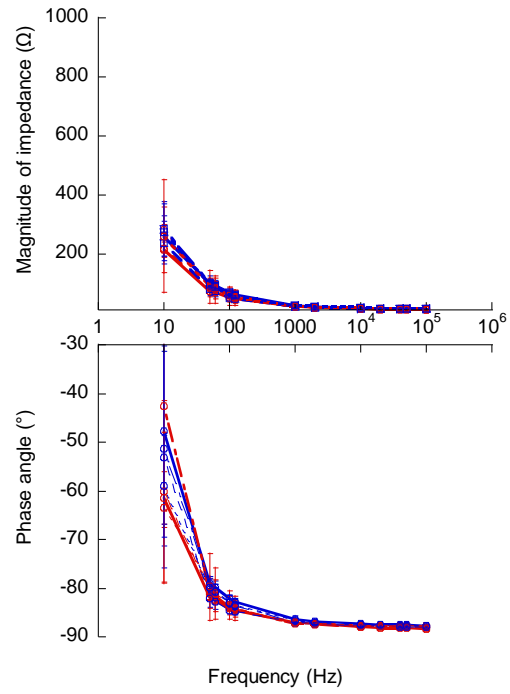

(i)

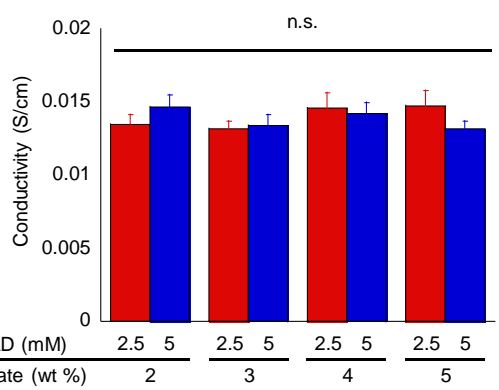

$\mathrm{V}=1.5 \mathrm{v}$

(ii)

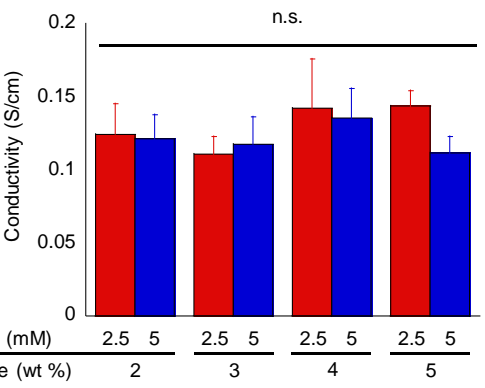

$V=2 v$

(iii)

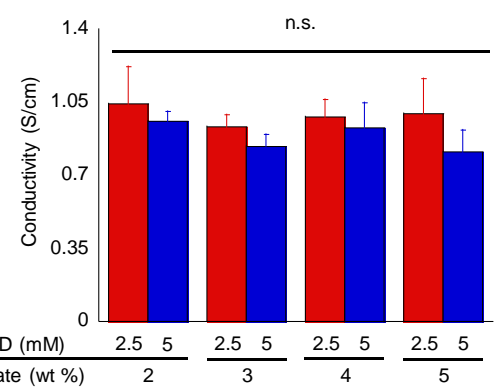

$\mathrm{V}=8 \mathrm{v}$

Figure 4.5. Hydrogel electrical properties are independent of hydrogel

formulation. (A). IV curve for different hydrogel formulations made from alginate (2$5 \mathrm{wt} \%)$ and crosslinked with AAD (2.5 \& $5 \mathrm{mM})$ with constant $0.26 \mathrm{wt} \%$ PEDOT. 8, $4,2,1.5,-1.5,-2,-4$ and $8 \mathrm{v}$ voltages were applied, and currents were read. (B). Impedance for different hydrogel formulations made from alginate (2- $5 \mathrm{wt} \%$ ) and crosslinked with AAD $(2.5 \& 5 \mathrm{mM})$ with constant 0.26 wt $\%$ PEDOT. Frequency was 
applied from 10 to $10^{5} \mathrm{~Hz}$ at $10 \mathrm{mv}$ voltage. (C) Conductivities for different hydrogel formulations made from alginate (2-5 wt $\%)$ and crosslinked with AAD (2.5 \& $5 \mathrm{wt}$ $\%$ ) with constant $0.26 \mathrm{wt} \%$ PEDOT at (i) $\mathrm{V}=1.5 \mathrm{v}$, (ii) $\mathrm{V}=2 \mathrm{v}$, and (iv) $\mathrm{V}=8 \mathrm{v}$. For all studies $\mathrm{N}=4$ and $\mathrm{n}$.s. indicates that no statistically significant difference was found $(\mathrm{p}>0.05)$

\subsubsection{Isolation between TRE rings}

One of the main issues with TCREs is the use of conductive paste, which is applied between the scalp and electrodes that partially shorts the electrode channels and affects the quality of signals. Additionally, conductive paste dries over time which affects the long-term stability of the system. To ensure isolation between the channels present in the TCRHE, impedance between the channels was measured. Furthermore, TCRHE with two heights $(2.5$ and $5 \mathrm{~mm}$ ) were fabricated to optimize the design of the system (Figures $4.6 \mathrm{~B} 1$ ). Impedance between two channels was measured at a time. Figures $4.6 \mathrm{~A}, 4.6 \mathrm{~B}$ and $4.6 \mathrm{C}$ demonstrated impedance between the middle ring to center disc, center disc to outer ring and middle ring to outer ring for TCRHE, indicating that the impedance between the channels were at least two orders of magnitude higher for these systems than TCRE with conductive paste. Higher impedance between channels is an indication of isolation between the rings (Figures 4.6 and $\mathrm{B} 1)$. 

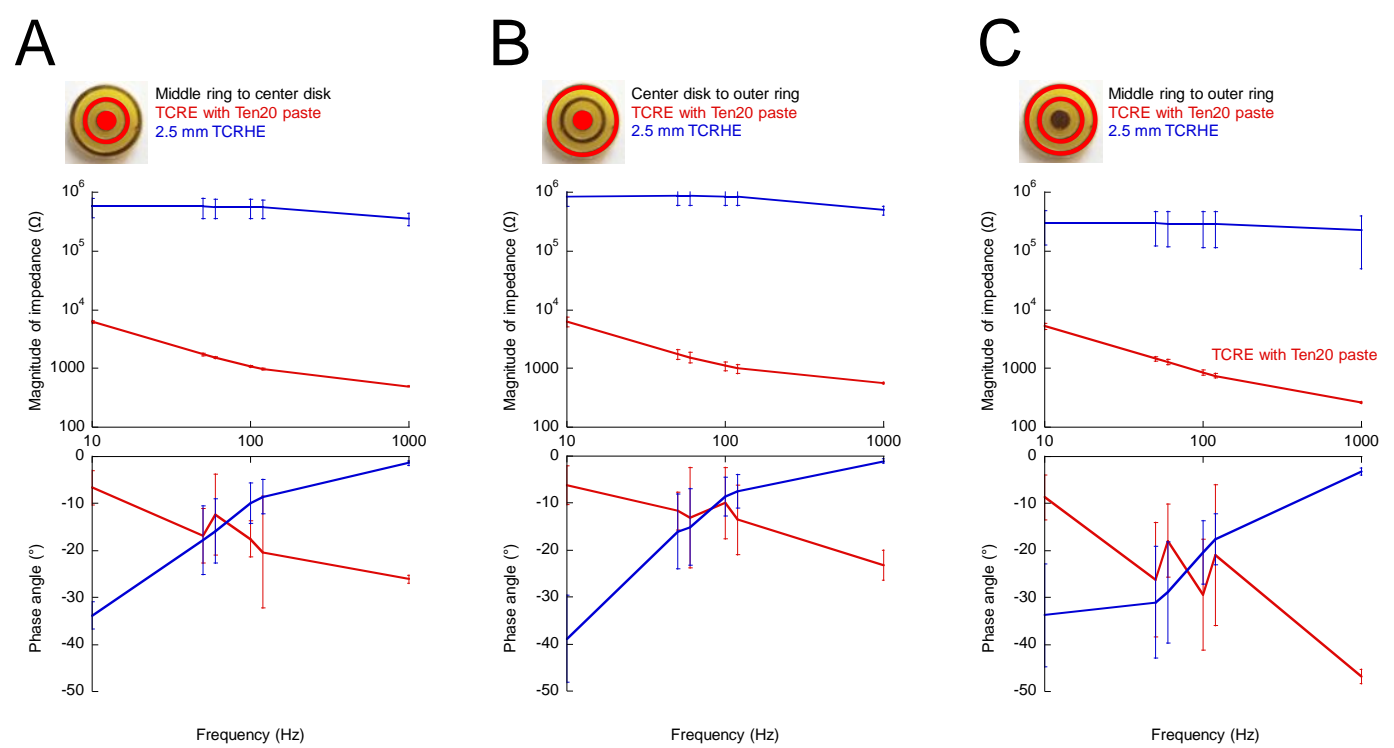

Figure 4.6. TCRHE exhibit much better isolation between channels in comparison to conductive paste, where high impedance between channels indicates isolation. (A) Isolation between middle ring to center disc. Red for TCRE with Ten20 paste and blue for $2.5 \mathrm{~mm}$ TCR hydrogel electrode (2.5 mm TCRHE) (2 wt $\%$ alginate, $2.5 \mathrm{mM} \mathrm{AAD}$ and $0.26 \mathrm{wt} \%$ PEDOT). (B) Isolation between center disc to outer ring. Red for TCRE with Ten20 paste and blue for $2.5 \mathrm{~mm}$ TCR hydrogel electrode (2.5 mm TCRHE) ( $2 \mathrm{wt} \%$ alginate, $2.5 \mathrm{mM} \mathrm{AAD}$ and $0.26 \mathrm{wt} \%$ PEDOT). (C) Isolation between outer ring to middle ring. Red for TCRE with Ten20 paste and blue for $2.5 \mathrm{~mm}$ TCR hydrogel electrode (2.5 mm TCRHE)) ( $2 \mathrm{wt} \%$ alginate, $2.5 \mathrm{mM}$ AAD and 0.26 wt \% PEDOT). Frequency was applied from 10 to $1000 \mathrm{~Hz}$ at $10 \mathrm{mv}$ voltage.

\subsubsection{EEG testing}

Volunteers (two male and two female) were involved in the study and signed IRB approved consent forms. To evaluate the performance of TCRHEs and in 
comparison, to TCREs, EEG testing was performed while volunteers wore the aforementioned electrodes. Figures 4.8 and B3 demonstrated that TCRHE exhibited better performance compared to TCRE with Ten20 paste, providing higher potential and power spectrum, especially while patient eyes were closed. Figures 4.7A and S3A show real-time recording and alpha rhythms, where the alpha rhythm is normal brain activity when conscious and relaxed, consisting of oscillations of $8-13 \mathrm{~Hz}$. Such rhythms can be detected from the occipital lobe while eyes are closed, and was clearly observed for all of the electrodes evaluated (2.5 and $5 \mathrm{~mm}$ TCRHE and TCRE with Ten20 paste). Results showed that $2.5 \mathrm{~mm}$ TCRHE exhibited higher amplitude in comparison to TCRE with Ten20 paste. Figures 4.7B and B2B show the power spectra of the electrodes while patients' eyes were closed, and the inset data showing the power spectrum for alpha waves clearly indicate that $2.5 \mathrm{~mm}$ TCRHE exhibited better performance in comparison to TCRE with Ten20 paste. Figures 4.7C and B3C show real-time EEG recordings, where alpha rhythms disappeared as soon as the eyes were opened. Figures 4.7D and B2D show the power spectra of TCRE and TCRHE while the volunteer's eyes were open. The data showed that TCRHE can not only record neural activity, but also have better performance than TCRE with Ten20 paste.

Moreover, Figure B2 shows that $2.5 \mathrm{~mm}$ TCRHE exhibited better performance than $5 \mathrm{~mm}$ TCRHE. Since impedance decreases with decreasing thickness, $2.5 \mathrm{~mm}$ TCRHE outperformed $5 \mathrm{~mm}$ TCRHE as it had lower impedance. In addition, the 2.5 $\mathrm{mm}$ TCHRE had a higher signal-to-noise ratio than the $5 \mathrm{~mm}$ TCRHE and TCRE with Ten20 paste at its peak. The increase in performance of the $2.5 \mathrm{~mm}$ TCRHE in comparison to the $5 \mathrm{~mm}$ TCRHE is caused by the differences in their impedance. It 
was also shown that by decreasing the electrode lead height, the signal-to-noise ratio could be further enhanced (Table 4.1). Since the $2.5 \mathrm{~mm}$ TCRHE exhibited better performance parameters and had higher signal-to-noise ratio than the $5 \mathrm{~mm}$ TCRHE the EEG longevity test was only performed using $2.5 \mathrm{~mm}$ TCRHE.
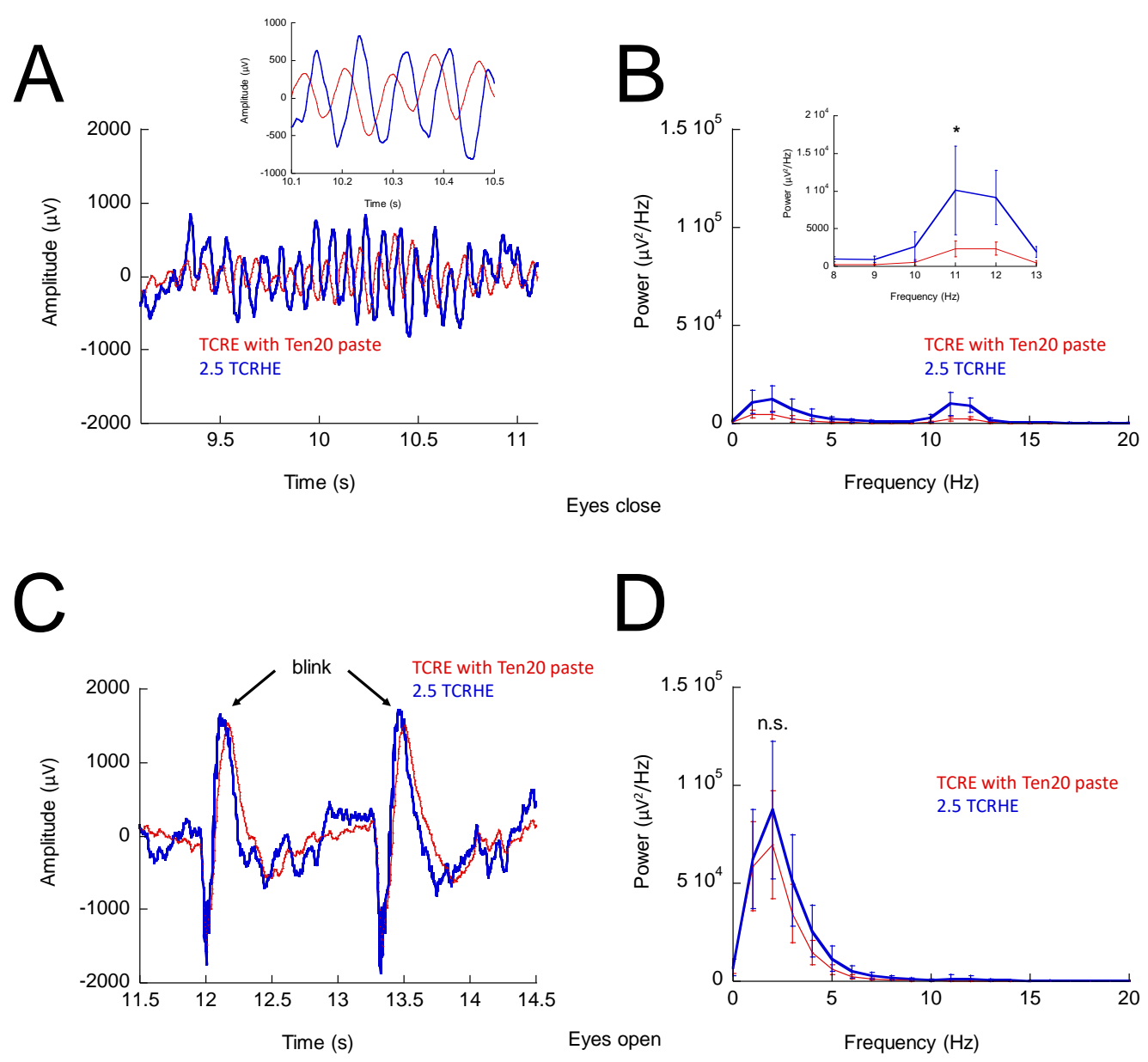

Figure 4.7. TCRHE have higher amplitude and significantly improves the power spectrum in comparison to TCRE with Ten20 paste. (A) EEG recording from occipital lobe using Ten20 paste (red) compared to hydrogel $(2.5 \mathrm{~mm}$ lead electrode (blue)) while subject eyes were closed. Inset: zoom-in EEG recording while eyes were closed. (B) Power spectrum from occipital lobe using Ten20 paste (red) compared to 
hydrogel (2.5 mm electrode lead (blue)) while subject eyes were closed. Inset: zoomin power spectra, highlighting the recording of alpha waves $(8-13 \mathrm{~Hz})$ only with eyes closed. (C) EEG recording from occipital lobe using Ten20 paste (red) compared to hydrogel (2.5 mm lead electrode (blue)) while subject eyes were opened. Alpha wave vanished as soon as eyes were opened. (D) Power spectrum from occipital lobe using Ten20 paste (red) compared to hydrogel $(2.5 \mathrm{~mm}$ lead electrode (blue) $)$ while the subject's eyes were open. Alpha waves disappeared as soon as eyes were opened. For all parts, $\mathrm{N}=4$ at 0 hour. * indicates p-values $<0.05$.

\subsubsection{EEG longevity test}

To characterize the durability of the electrodes, EEG testing was performed after the subject wore electrodes for 4 hours continuously, while moving around freely. As seen in Figure 4.8, alpha waves were detected and the TCRHE had a higher signal amplitude compared to TCRE with Ten20 paste. Figure 4.8B shows the, displayed power spectra while the subject's eyes were closed, and the inset illustrates the alpha wave power spectra, indicating that TCRHE had higher power than TCRE with Ten20 paste after $4 \mathrm{~h}$. Figure $4.8 \mathrm{D}$ (power spectrum) show data where the subject's eyes were open, which showed that TCRHE had a better performance than TCRE. 

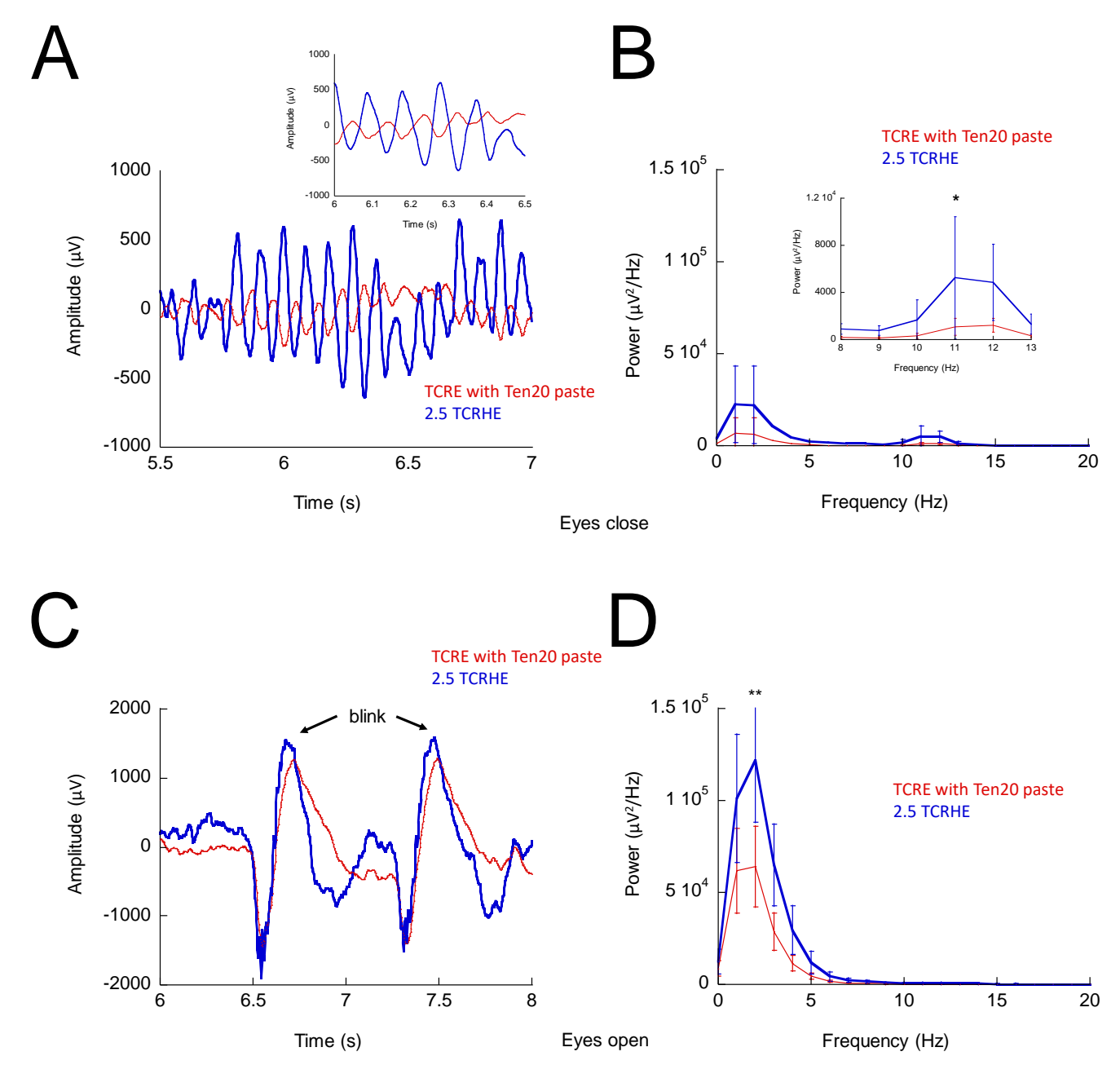

Figure 4.8. TCRHE have higher amplitude and significantly improve the power spectrum comparing to TCRE with Ten20 paste. (A) EEG recording from occipital lobe using Ten20 paste (red) compared to hydrogel $2.5 \mathrm{~mm}$ lead electrode (blue) while subject eyes were closed. Inset: zoom-in EEG recording while eyes were closed. (B) Power spectrum from occipital lobe using Ten20 paste (red) compared to hydrogel 2.5 mm electrode lead (blue) while subject eyes were closed. Inset: zoom-in power spectra, highlighting the recording of alpha waves $(8-13 \mathrm{~Hz})$ only with eyes closed. (C) EEG recording from occipital lobe using Ten20 paste (red) compared to hydrogel $2.5 \mathrm{~mm}$ lead electrode (blue), while subject eyes were opened. Alpha waves 
disappeared as soon as eyes opened. (D) Power spectrum from occipital lobe using Ten20 paste (red) compared to hydrogel $2.5 \mathrm{~mm}$ lead electrode (blue) while subject eyes were open. Alfa waves disappeared as soon as eyes opened. For all parts, $\mathrm{N}=4$ at 4 hours. * indicates p-values $<0.05, * *$ indicates p-values $<0.01$.

Table 4.1. Signal-to-noise ratio of electrodes at $0 \mathrm{~h}$ and $4 \mathrm{~h}$ for alpha wave $(8-13$ Hz). Signal-to-noise ratio for alpha oscillation for TCRE with Ten20 paste and 2.5 $\mathrm{mm}$ TCRHE at 0 hour and 4 hour and for $5 \mathrm{~mm}$ TCRHE at $0 \mathrm{~h}$.

\begin{tabular}{|c|c|c|c|c|c|c|}
\hline $\begin{array}{l}\text { freque } \\
\text { ncy }\end{array}$ & Ten2 & paste & $2.5 \mathrm{~mm}$ & ГCRHE & $5 \mathrm{~mm}$ TCR & \\
\hline & $\mathbf{0 h}$ & $4 h$ & $\mathbf{O h}$ & $4 \mathrm{~h}$ & Oh & $4 h$ \\
\hline 8 & $0.66 \pm 0.11$ & $1.26 \pm 0.85$ & $0.87 \pm 0.17$ & $1.81 \pm 0.79$ & $1.03 \pm 0.28$ & - \\
\hline 9 & $0.96 \pm 0.37$ & $1.15 \pm 0.54$ & $1.25 \pm 0.2$ & $1.46 \pm 0.55$ & $1.07 \pm 0.27$ & - \\
\hline 10 & $3.22 \pm 4.01$ & $2.53 \pm 0.54$ & $5.61 \pm 1.23$ & $2.72 \pm 0.98$ & $2.10 \pm 0.51$ & - \\
\hline 11 & $19.38 \pm 2.38$ & $11.65 \pm 3.57$ & $21.16 \pm 1.77$ & $11.44 \pm 2.35$ & $11.69 \pm 2.95$ & - \\
\hline 12 & $21.46 \pm 2.38$ & $14.48 \pm 2.68$ & $22.96 \pm 8.13$ & $11.15 \pm 1.46$ & $14.27 \pm 5.62$ & - \\
\hline 13 & $6.20 \pm 1.65$ & $5.06 \pm 0.66$ & $5.61 \pm 1.90$ & $4.61 \pm 1.04$ & $4.64 \pm 3.21$ & - \\
\hline
\end{tabular}

Figure 4.9 shows the performance of TCRHE and TCRE with Ten20 paste over time at $8-13 \mathrm{~Hz}$ while eyes were closed (alpha waves). The conductive hydrogel and electrode design enhanced signal amplitude and maintained a steady interface between skin and the electrode. There was no statistically significant difference in 
TCRHE power after 4 hours, whereas the power decreased over time for TCRE with Ten20 paste.

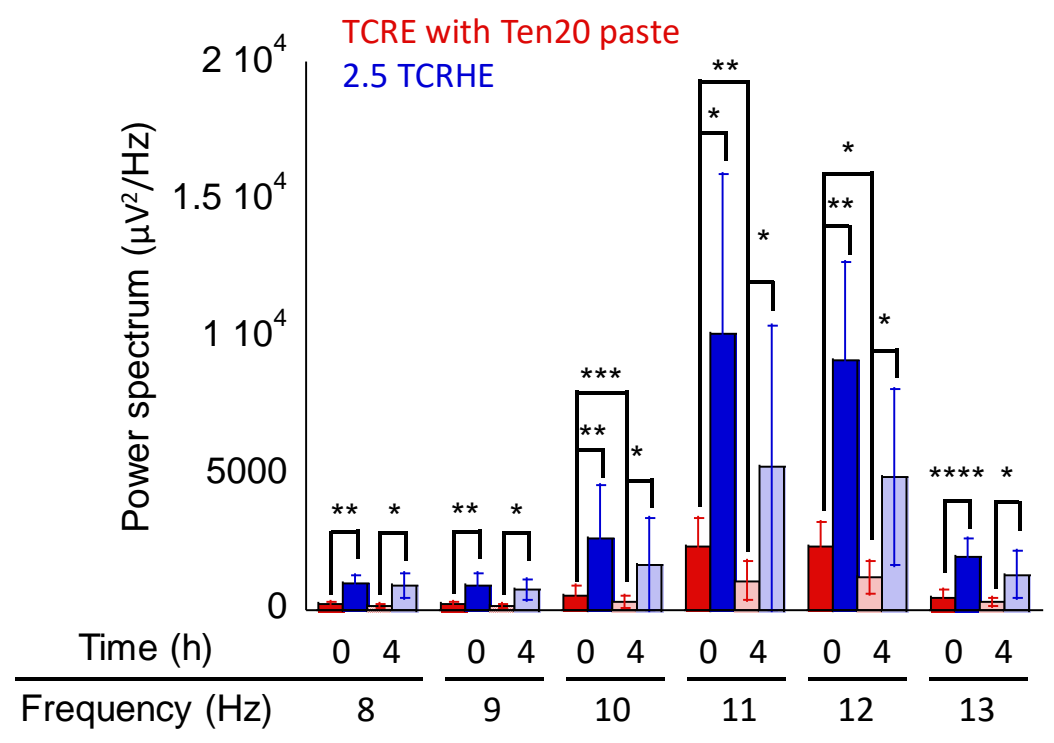

Figure 4.9. TCRHE had higher power spectrum compared to TCRE with Ten20 paste over time. 8 - $13 \mathrm{~Hz}$ (alpha waves) while subject's eyes were closed. $\mathrm{N}=4$ for all parts. $*$ indicates p-values $<0.05$, ** indicates $\mathrm{p}$-values $<0.01$, *** indicates $\mathrm{p}$ values $<0.001$ and $* * * *$ indicates $\mathrm{p}$-values $<0.0001$.

The aim of this work was to develop a novel conductive polymer hydrogel serving as an interface between an electrode and skin tissue combining the beneficial properties of hydrogels with excellent electrical properties of PEDOT. With such interfaces, it is important to consider a design which provides a stable, durable interface with the tissue. To achieve this goal, we developed a fabrication method of conductive hydrogels where the conductive hydrogel was made using commercially available PEDOT. With this method of fabrication, conductive hydrogels were made 
with tunable mechanics and favorable electrical properties for the described application. This method of fabrication can be adapted for a wide range of negatively and neutrally charged polymers.

There has been significant effort to develop electrodes that eliminate the use of a conductive interface (dry electrodes). Some of the most attractive features of dry electrodes are their robustness and ability to adapt to body curvature. On the other hand, dry electrodes have high impedance and do not provide a good connection between the electrode and skin, resulting in poor signal quality. These features limit the use of dry electrodes and thus conductive paste is used to overcome such limitations (wet electrodes). Conductive paste dries over time, which can affect the quality of recorded signals $[43,44]$. Recent advances in the integration of materials and electronic systems have resulted in a new generation of dry electrodes which integrate conductive hydrogels [45-47] and conductive polymers [48] within the electrodes. PEDOT:PSS was used as conductive interface to fabricate hydrogel-based dry electrodes. These designs, different in their natures (electrode type and conductive layer), improved the quality and stability of EEG signals, and enhanced the impedance and signal-to-noise ratio of electrodes. Our conductive hydrogels are robust, similar to dry electrodes, and provide a good connection with skin tissue as wet electrodes do.

\subsection{Conclusion}

In conclusion, we developed a new design to integrate a conductive interface within an electrode that maintained a good connection with skin for successful EEG recordings. Electrodes with alg-PEDOT hydrogel interfaces were optimized with a 
thickness that resulted in stronger signal amplitudes than that of regular TCREs with conductive paste. Additionally, the hydrogel-based electrodes maintained their integrity over time. Moreover, by decreasing the electrode design's signal-to-noise ratio will be improved. Overall, this work shows a new fabrication approach that can be used to integrate a conductive hydrogel interface with electrodes, creating new designs that enhance spatial resolution for high quality electroencephalography.

\section{Supporting information}

Supporting information can be found in Appendix B.

\section{Acknowledgements}

This research was supported by NSF (RII Track-2 FEC 1539068), 3M Company Nontenure Faculty Award (32976949) and start-up support from the University of Rhode Island, College of Engineering.

The authors like to thank Tanya Wang for her help with 3D printing. Preston Steele for training EEG test. Brandon Williams, Lynne McCane and Jason Mercier for volunteering for EEG testing. 


\subsection{References}

[1] A. Horvath, A. Szucs, G. Csukly, A. Sakovics, G. Stefanics, A. Kamondi, EEG and ERP biomarkers of Alzheimer's disease: a critical review, Front Biosci (Landmark Ed) 23 (2018) 183-220.

[2] Y. Yokoi, Z. Narita, T. Sumiyoshi, Transcranial Direct Current Stimulation in Depression and Psychosis: A Systematic Review, Clin EEG Neurosci (2017) 1550059417732247.

[3] B.A. Coffman, S.M. Haigh, T.K. Murphy, J. Leiter-Mcbeth, D.F. Salisbury, Reduced auditory segmentation potentials in first-episode schizophrenia, Schizophr Res (2017).

[4] S.F. Schoch, E. Werth, R. Poryazova, T.E. Scammell, C.R. Baumann, L.L. Imbach, Dysregulation of Sleep Behavioral States in Narcolepsy, Sleep (2017).

[5] C.S. Williams, M.C. Spitz, J.F. Foley, L.K. Weaver, A.S. Lindblad, M.R. Wierzbicki, Baseline EEG abnormalities in mild traumatic brain injury from the BIMA study, Undersea Hyperb Med 43(5) (2016) 521-530.

[6] B.L. Edlow, C. Chatelle, C.A. Spencer, C.J. Chu, Y.G. Bodien, K.L. O'Connor, R.E. Hirschberg, L.R. Hochberg, J.T. Giacino, E.S. Rosenthal, O. Wu, Early detection of consciousness in patients with acute severe traumatic brain injury, Brain 140(9) (2017) 2399-2414.

[7] F. Zubler, A. Steimer, R. Kurmann, M. Bandarabadi, J. Novy, H. Gast, M. Oddo, K. Schindler, A.O. Rossetti, EEG synchronization measures are early outcome 
predictors in comatose patients after cardiac arrest, Clin Neurophysiol 128(4) (2017) 635-642.

[8] R. Sutter, R.D. Stevens, P.W. Kaplan, Clinical and imaging correlates of EEG patterns in hospitalized patients with encephalopathy, J Neurol 260(4) (2013) 1087-98.

[9] M. Teplan, Fundamentals of EEG measurements, MEASUREMENT SCIENTIFIC REVIEW 2 (2002).

[10] S.A. Park, H.J. Hwang, J.H. Lim, J.H. Choi, H.K. Jung, C.H. Im, Evaluation of feature extraction methods for EEG-based brain-computer interfaces in terms of robustness to slight changes in electrode locations, Med Biol Eng Comput 51(5) (2013) 571-9.

[11] D.J. McFarland, The advantages of the surface Laplacian in brain-computer interface research, Int J Psychophysiol 97(3) (2015) 271-6.

[12] D.B. Salisbury, T.D. Parsons, K.R. Monden, Z. Trost, S.J. Driver, Braincomputer interface for individuals after spinal cord injury, Rehabil Psychol 61(4) (2016) 435-441.

[13] P.L. Nunez, R.B. Silberstein, P.J. Cadusch, R.S. Wijesinghe, A.F. Westdorp, R. Srinivasan, A theoretical and experimental study of high resolution EEG based on surface Laplacians and cortical imaging, Electroencephalography and Clinical Neurophysiology 90(1) (1994) 40-57.

[14] W.G. Besio, K. Koka, R. Aakula, W. Dai, Tri-polar concentric ring electrode development for laplacian electroencephalography, Ieee T Bio-Med Eng 53(5) (2006) 926-33. 
[15] S.K. Law, P.L. Nunez, R.S. Wijesinghe, High-resolution EEG using spline generated surface Laplacians on spherical and ellipsoidal surfaces, Ieee T BioMed Eng 40(2) (1993) 145-53.

[16] B. He, J. Lian, G. Li, High-resolution EEG: a new realistic geometry spline Laplacian estimation technique, Clinical Neurophysiology 112(5) (2001) 845852.

[17] C. Carvalhaes, J.A. de Barros, The surface Laplacian technique in EEG: Theory and methods, Int J Psychophysiol 97(3) (2015) 174-88.

[18] D. Farina, C. Cescon, Concentric-ring electrode systems for noninvasive detection of single motor unit activity, Ieee T Bio-Med Eng 48(11) (2001) 1326-34.

[19] W. Besio, R. Aakula, K. Koka, W. Dai, Development of a Tri-polar Concentric Ring Electrode for Acquiring Accurate Laplacian Body Surface Potentials, Annals of Biomedical Engineering 34(3) (2006) 426-435.

[20] P. Tallgren, S. Vanhatalo, K. Kaila, J. Voipio, Evaluation of commercially available electrodes and gels for recording of slow EEG potentials, Clin Neurophysiol 116(4) (2005) 799-806.

[21] L. Pan, G. Yu, D. Zhai, H.R. Lee, W. Zhao, N. Liu, H. Wang, B.C. Tee, Y. Shi, Y. Cui, Z. Bao, Hierarchical nanostructured conducting polymer hydrogel with high electrochemical activity, Proc Natl Acad Sci U S A 109(24) (2012) 928792. 
[22] S. Naficy, J.M. Razal, G.M. Spinks, G.G. Wallace, P.G. Whitten, Electrically Conductive, Tough Hydrogels with $\mathrm{pH}$ Sensitivity, Chemistry of Materials 24(17) (2012) 3425-3433.

[23] G.A. Saracino, D. Cigognini, D. Silva, A. Caprini, F. Gelain, Nanomaterials design and tests for neural tissue engineering, Chem Soc Rev 42(1) (2013) $225-62$.

[24] G.L. Mario Cheong, K.S. Lim, A. Jakubowicz, P.J. Martens, L.A. Poole-Warren, R.A. Green, Conductive hydrogels with tailored bioactivity for implantable electrode coatings, Acta Biomater 10(3) (2014) 1216-26.

[25] J.B. Leach, A.K. Achyuta, S.K. Murthy, Bridging the Divide between Neuroprosthetic Design, Tissue Engineering and Neurobiology, Front Neuroeng 2(18) (2010) 18.

[26] U.A. Aregueta-Robles, A.J. Woolley, L.A. Poole-Warren, N.H. Lovell, R.A. Green, Organic electrode coatings for next-generation neural interfaces, Frontiers in Neuroengineering 7(15) (2014).

[27] Y. Zhao, B. Liu, L. Pan, G. Yu, 3D nanostructured conductive polymer hydrogels for high-performance electrochemical devices, Energy \& Environmental Science 6(10) (2013).

[28] N.K. Guimard, N. Gomez, C.E. Schmidt, Conducting polymers in biomedical engineering, Progress in Polymer Science 32(8-9) (2007) 876-921.

[29] M.R. Abidian, D.H. Kim, D.C. Martin, Conducting-Polymer Nanotubes for Controlled Drug Release, Adv Mater 18(4) (2006) 405-409. 
[30] M.R. Abidian, D.C. Martin, Experimental and theoretical characterization of implantable neural microelectrodes modified with conducting polymer nanotubes, Biomaterials 29(9) (2008) 1273-83.

[31] P. Huang, W. Chen, L. Yan, An inorganic-organic double network hydrogel of graphene and polymer, Nanoscale 5(13) (2013) 6034-9.

[32] S. Sekine, Y. Ido, T. Miyake, K. Nagamine, M. Nishizawa, Conducting polymer electrodes printed on hydrogel, J Am Chem Soc 132(38) (2010) 13174-5.

[33] W. Cho, J. Wu, B.S. Shim, W.F. Kuan, S.E. Mastroianni, W.S. Young, C.C. Kuo, T.H. Epps, 3rd, D.C. Martin, Synthesis and characterization of bicontinuous cubic poly(3,4-ethylene dioxythiophene) gyroid (PEDOT GYR) gels, Phys Chem Chem Phys 17(7) (2015) 5115-23.

[34] S.R. Benbadis, E. O'Neill, W.O. Tatum, L. Heriaud, Outcome of prolonged video-EEG monitoring at a typical referral epilepsy center, Epilepsia 45(9) (2004) 1150-3.

[35] M. Pawlaczyk, M. Lelonkiewicz, M. Wieczorowski, Age-dependent biomechanical properties of the skin, Postepy Dermatol Alergol 30(5) (2013) $302-6$.

[36] Y. Zhao, S. Chen, J. Hu, J. Yu, G. Feng, B. Yang, C. Li, N. Zhao, C. Zhu, J. Xu, Microgel-Enhanced Double Network Hydrogel Electrode with High Conductivity and Stability for Intrinsically Stretchable and Flexible All-GelState Supercapacitor, ACS Appl Mater Interfaces 10(23) (2018) 19323-19330.

[37] T. Dai, X. Qing, Y. Lu, Y. Xia, Conducting hydrogels with enhanced mechanical strength, Polymer 50(22) (2009) 5236-5241. 
[38] T. Dai, X. Qing, H. Zhou, C. Shen, J. Wang, Y. Lu, Mechanically strong conducting hydrogels with special double-network structure, Synthetic Metals 160(7-8) (2010) 791-796.

[39] T. Dai, Z. Shi, C. Shen, J. Wang, Y. Lu, Self-strengthened conducting polymer hydrogels, Synthetic Metals 160(9-10) (2010) 1101-1106.

[40] M. Sasaki, B.C. Karikkineth, K. Nagamine, H. Kaji, K. Torimitsu, M. Nishizawa, Highly conductive stretchable and biocompatible electrode-hydrogel hybrids for advanced tissue engineering, Adv Healthc Mater 3(11) (2014) 1919-27.

[41] L. Manola, B.H. Roelofsen, J. Holsheimer, E. Marani, J. Geelen, Modelling motor cortex stimulation for chronic pain control: electrical potential field, activating functions and responses of simple nerve fibre models, Med Biol Eng Comput 43(3) (2005) 335-43.

[42] S. Gabriel, R.W. Lau, C. Gabriel, The dielectric properties of biological tissues: II. Measurements in the frequency range $10 \mathrm{~Hz}$ to $20 \mathrm{GHz}$, Phys Med Biol 41(11) (1996) 2251-69.

[43] M.A. Lopez-Gordo, D. Sanchez-Morillo, F. Pelayo Valle, Dry EEG electrodes, Sensors (Basel) 14(7) (2014) 12847-70.

[44] G. Li, S. Wang, Y.Y. Duan, Towards gel-free electrodes: A systematic study of electrode-skin impedance, Sensors and Actuators B: Chemical 241 (2017) 1244-1255.

[45] K. Nagamine, S. Chihara, H. Kai, H. Kaji, M. Nishizawa, Totally shapeconformable electrode/hydrogel composite for on-skin electrophysiological measurements, Sensors and Actuators B: Chemical 237 (2016) 49-53. 
[46] N.A. Staples, J.A. Goding, A.D. Gilmour, K.Y. Aristovich, P. Byrnes-Preston, D.S. Holder, J.W. Morley, N.H. Lovell, D.J. Chew, R.A. Green, Conductive Hydrogel Electrodes for Delivery of Long-Term High Frequency Pulses, Front Neurosci 11 (2017) 748.

[47] C. Kleber, M. Bruns, K. Lienkamp, J. Ruhe, M. Asplund, An interpenetrating, microstructurable and covalently attached conducting polymer hydrogel for neural interfaces, Acta Biomater 58 (2017) 365-375.

[48] K. Wang, U. Parekh, T. Pailla, H. Garudadri, V. Gilja, T.N. Ng, Stretchable Dry Electrodes with Concentric Ring Geometry for Enhancing Spatial Resolution in Electrophysiology, Adv Healthc Mater 6(19) (2017).

[49] Y. Ye-Lin, E. Senet, G. Prats-Boluda, E. Garcia-Breijo, J.V. Lidon, J. GarciaCasado, Tripolar Flexible Concentric Ring Electrode Printed with Inkjet Technology for ECG Recording, In: Roa Romero L. (eds) XIII Mediterranean Conference on Medical and Biological Engineering and Computing 2013 41(IFMBE Proceedings) (2013) 1579-1582.

[50] C.-C. Lu, P.P. Tarjan, Pasteless, Active, Concentric Ring Sensors for Directly Obtained Laplacian Cardiac Electrograms, Journal of Medical and Biological Engineering 22(4) (2002) 199-203. 


\section{CHAPTER 5}

\section{CONCLUSIONS AND FUTURE WORK}

\subsection{Conclusions}

This dissertation focused on the fabrication, development, optimization and characterization of conductive hydrogels. Several conductive hydrogels systems were made using commercially available conductive polymer. The method of hydrogel production was fast and does not require specific instruments. Additionally, both natural and synthetic polymers were used to fabricate conductive hydrogels with this method, demonstrating the versatility in the method itself.

In the third chapter, we used the synthetic polymer polyacrylic acid to make hydrogels at room and sub-freezing temperatures in order to fabricate conductive hydrogels with different morphologies (macroporous and nanoporous structures). The macroporous cryogel exhibited shape memory properties, making them suitable for injection. Furthermore, by changing the formulation of the cryogels wide range mechanical properties; young's modulus between $0.2-20 \mathrm{kPa}$, high strain of failure (they could endure $90 \%$ compressive strain) and excellent toughness (above $2 \mathrm{~kJ} / \mathrm{m}^{3}$ ), were achieved. These properties are crucial to ensure the robustness and durability of these materials. Additionally, the hydrogels exhibited sufficient conductivity to record neural activity. Finally, the materials were biocompatible, and were loaded with and successfully delivered protein to reduce inflammation. 
In chapter 4, we used the natural polymer alginate to make conductive hydrogels to be incorporated into electrodes. In this work, the goal was to integrate conductive hydrogels into a tripolar concentric electrode configuration to enhance channel isolation, and to fabricate electrodes that can record neural activity for prolonged periods of time. To do so, we used nanoporous hydrogels that could hold a large amount of water within their network. The resulting alginate-PEDOT (algPEDOT) hydrogels exhibited a high strain of failure (above $80 \%$ ) and great toughness (above $2 \mathrm{~kJ} / \mathrm{m}^{3}$ ). Integration of the alg-PEDOT hydrogel into a tripolar concentric ring configuration significantly enhanced channel isolation (about two order of magnitude). In addition, the optimized electrode design amplified neural activity recorded with the hydrogel-based electrodes in comparison with tripolar concentric ring electrode (TCREs). The optimized design enhanced the signal-to-noise ratio of the electrode, and this electrode design overcomes the limitation of using conductive paste. In addition, the given system was found to be robust and can adapt to body curvature, allowing the system to be more advantageous to previously-described designs.

\subsection{Future work}

This dissertation demonstrated a fast and easily accessible method for the fabrication of conductive hydrogels. Many opportunities exist to conduct further research with these materials, which may include, but is not limited to:

- Investigation of localized protein and drug delivery using cryogels by investigating different types of drugs and proteins that could be delivered by 
this system, which would also involve investigating the appropriate electric and polarity properties for safe drug delivery.

- Optimizing the mold design for TCREs by fabricating electrodes and molds as one piece. In this manner, the electrode lead height could be reduced and the signal-to-noise ratio could be further enhanced.

- With the capabilities of the enhanced special resolution of TCREs and conductive hydrogels capabilities to be fabricated in any design, targeted drug delivery and multi-drug delivery using tripolar concentric ring hydrogel electrodes could be further investigated. 


\section{APPENDIX A}

\section{Supplementary Materials for Chapter 3}

Graphical abstract
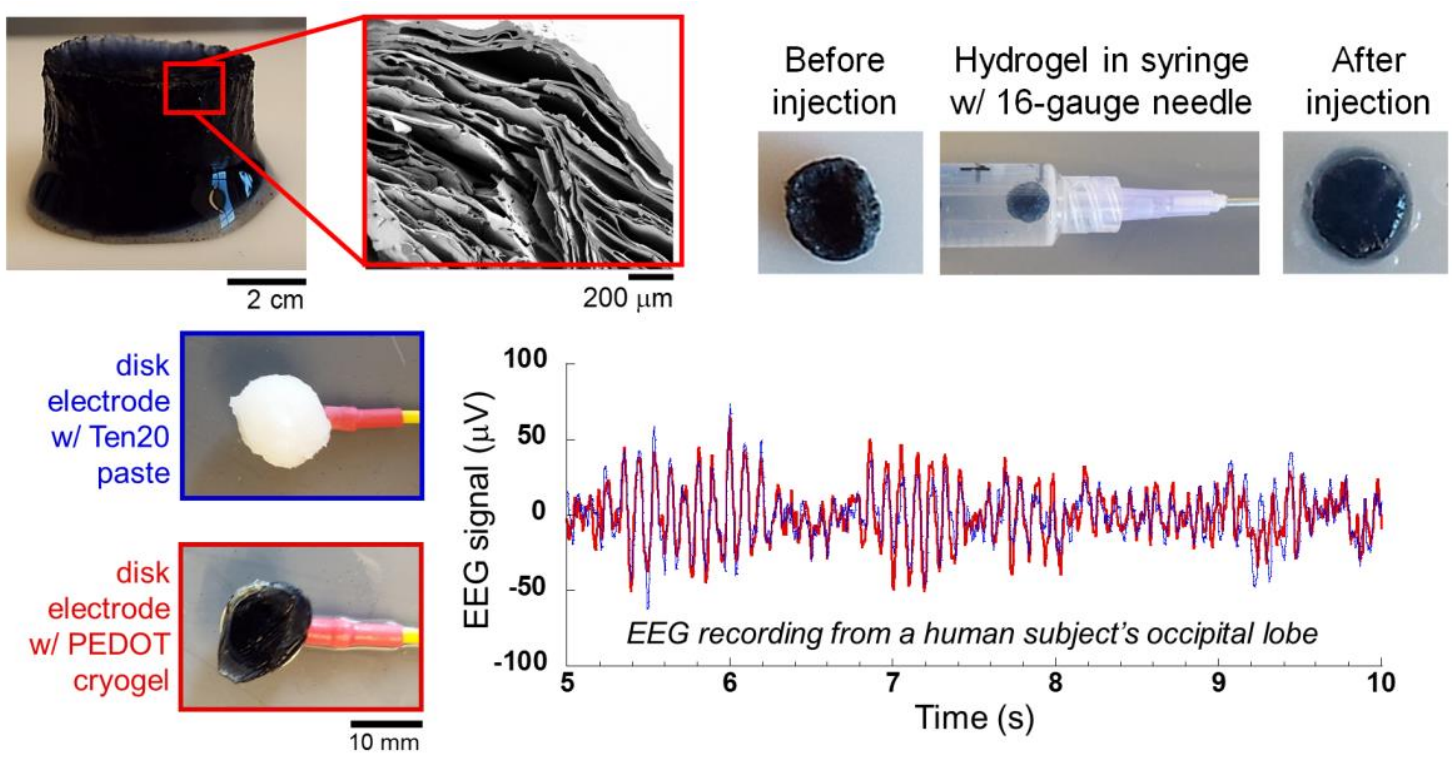

$200 \mu \mathrm{m}$

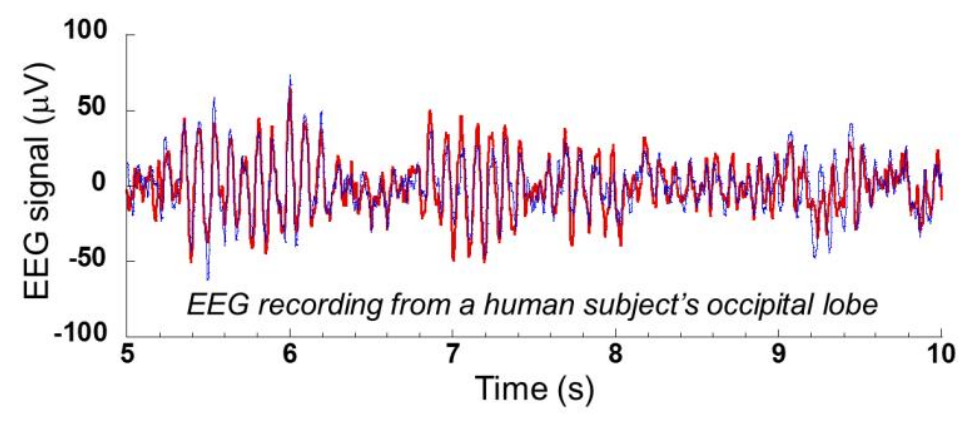

PEDOT-integrated cryogels exhibit porous, soft and highly compressible mechanics and can electrically transmit neural signals. PEDOT cryogels exhibit macroporous structures that enhance softness and compressibility which enables injection through a 16-guage needle. Cryogel conductivity is sufficient to record EEG signals at levels similar to traditional conductive pastes. 
(a)

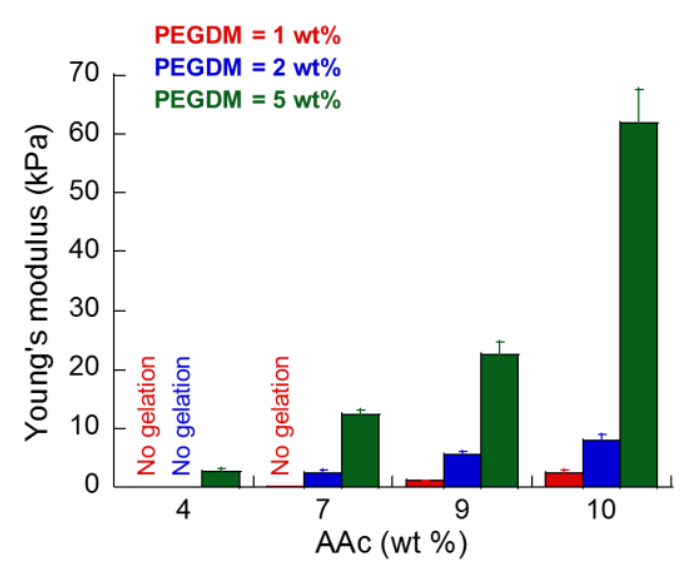

(b)

\begin{tabular}{|c|c|c|c|c|c|c|c|c|c|c|c|c|}
\hline & \multirow[b]{2}{*}{ wt $\%$ PEGDM } & \multicolumn{2}{|c|}{$4 \mathrm{wt} \%$ AAc } & \multicolumn{3}{|c|}{$7 \mathrm{wt} \%$ AAc } & \multicolumn{3}{|c|}{9 wt $\%$ AAc } & \multicolumn{3}{|c|}{$10 w t \%$ AAc } \\
\hline & & 1 wt $\% \quad 2$ wt $\%$ & $5 \mathrm{wt} \%$ & $1 \mathrm{wt} \%$ & $2 w t \%$ & 5 wt $\%$ & $1 \mathrm{wt} \%$ & $2 w t \%$ & 5 wt $\%$ & $1 \mathrm{wt} \%$ & 2 wt $\%$ & $5 \mathrm{wt} \%$ \\
\hline \multirow{3}{*}{$4 w t \%$ AAc } & $1 w t \%$ & & & & & & & & & & & \\
\hline & $2 w t \%$ & & & & & & & & & & & \\
\hline & 5 wt $\%$ & & & & n.s. & $\star \star \star \star \star$ & n.s. & n.s. & $\star * \star * *$ & n.s. & ** & 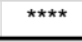 \\
\hline \multirow{3}{*}{$7 \mathrm{wt} \%$ AAc } & $1 \mathrm{wt} \%$ & & & & & & & & & & & \\
\hline & 2 wt $\%$ & & n.s. & & & $\star \star \star \star \star *$ & n.s. & n.s. & $* * * *$ & n.s. & ** & $\star * * * *$ \\
\hline & 5 wt $\%$ & & 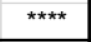 & & $\star \star \star * *$ & & $\star \star \star \star *$ & $\star \star \star *$ & $\star \star \star \star *$ & $\star * * * *$ & * & 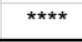 \\
\hline \multirow{3}{*}{9 wt $\%$ AAc } & $1 \mathrm{wt} \%$ & & n.s. & & n.s. & $\star \star \star \star \star$ & & * & $\star \star \star \star \star *$ & n.s. & $\star \star \star *$ & $\star \star \star \star \star *$ \\
\hline & $2 w t \%$ & & n.s. & & n.s. & $\star \star * *$ & * & & $\star \star \star \star *$ & n.s. & n.s. & $\star * * *$ \\
\hline & 5 wt $\%$ & & 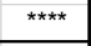 & & 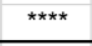 & 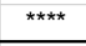 & $\star \star \star \star *$ & $\star \star \star \star *$ & & $\star \star \star \star *$ & 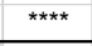 & 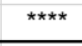 \\
\hline \multirow{3}{*}{$10 w t \%$ AAc } & $1 \mathrm{wt} \%$ & & n.s. & & n.s. & $\star * \star * *$ & n.s. & n.s. & $* * * *$ & & ** & $* * * *$ \\
\hline & $2 w t \%$ & & $\star \star$ & & $\star *$ & * & $\star \star \star$ & n.s. & 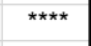 & $\star \star$ & & 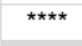 \\
\hline & 5 wt $\%$ & & $\star \star \star \star \star$ & & $\star \star \star \star \star$ & $\star \star \star \star \star$ & $\star \star \star \star$ & 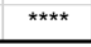 & $\star \star \star \star \star$ & $\star \star \star \star *$ & 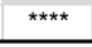 & \\
\hline
\end{tabular}

Figure A1. Nanoporous PEDOT/pAAc hydrogels exhibit soft and tunable

mechanics. (a) Compressive modulus vs. acrylic acid concentration for gels crosslinked with $1 \mathrm{wt} \%$ (red), $2 \mathrm{wt} \%$ (blue), and $5 \mathrm{wt} \%$ (green) PEGDM. "No gelation" indicates that there was not enough polymer and crosslinker to form a 3D gel structure and therefore no measurement could be taken. (b) Table summarizing statistically significant differences of moduli between different hydrogel formulations. $\mathrm{N}=4$. 

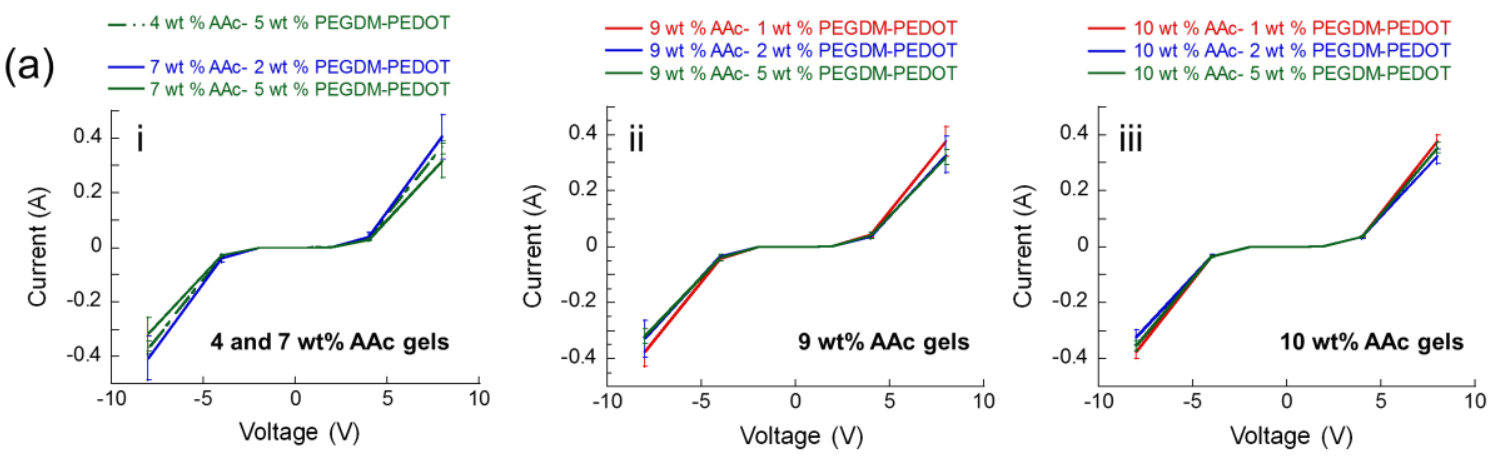

(b)
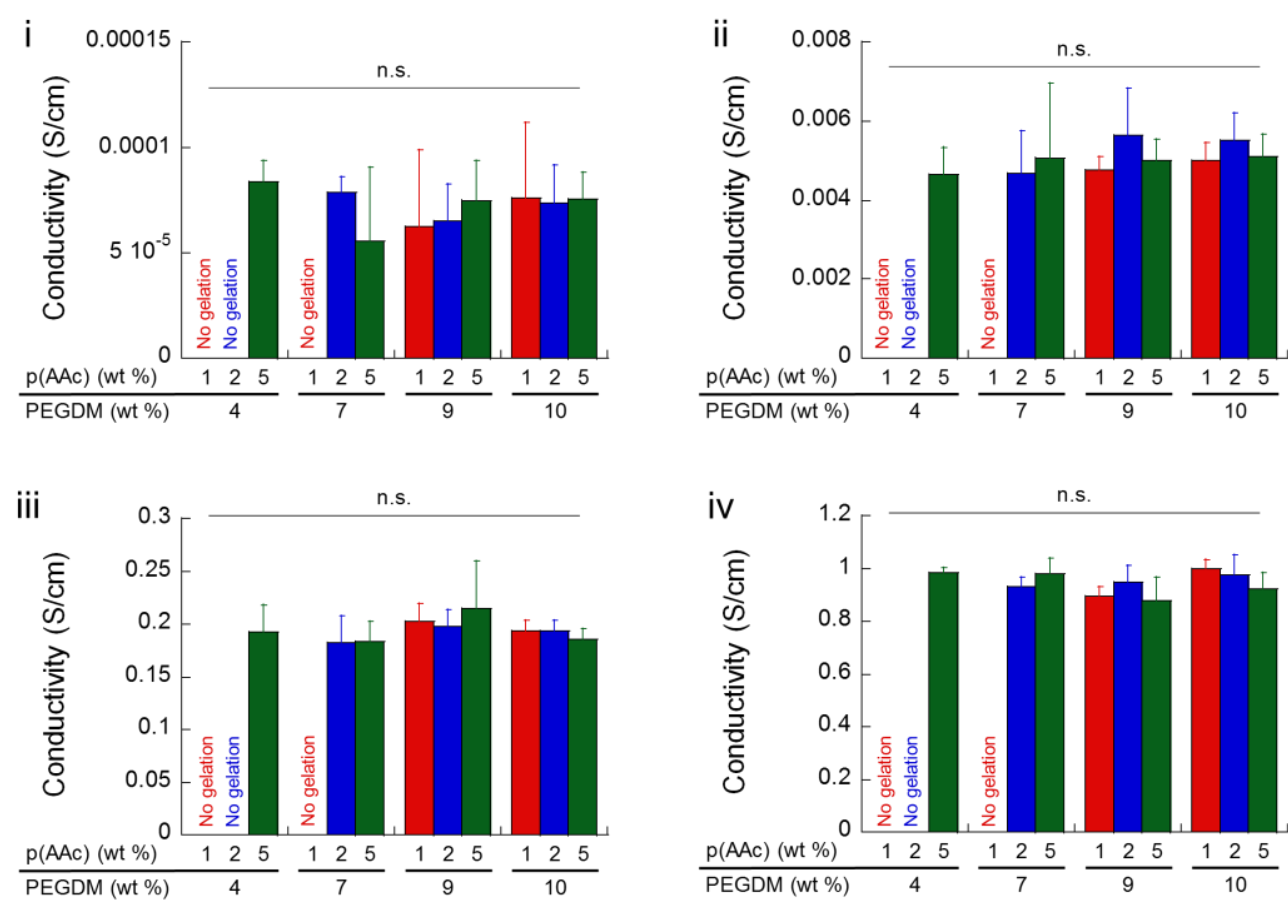

Figure A2. The electrical properties of hydrogels are not significantly impacted by acrylic acid and PEGDM crosslinking concentration. (a) I-V curves for gels composed 0.26 wt \% PEDOT and (i) 4 and 7 wt $\%$ AAc, (ii) 9 wt $\%$ AAc, and (iii) 10 wt\% AAc. (b) Computed DC electrical conductivity for various $0.26 \mathrm{wt} \%$ PEDOT hydrogel formulations when excited using (i) $0.5 \mathrm{~V}$, (ii) $2 \mathrm{~V}$, (iii) $4 \mathrm{~V}$, and (iv) $8 \mathrm{~V}$. n.s. indicates no statistically significant differences. $\mathrm{N}=4$. Note that some gel formulations lacked sufficient polymer and crosslinker to form stable gels. 

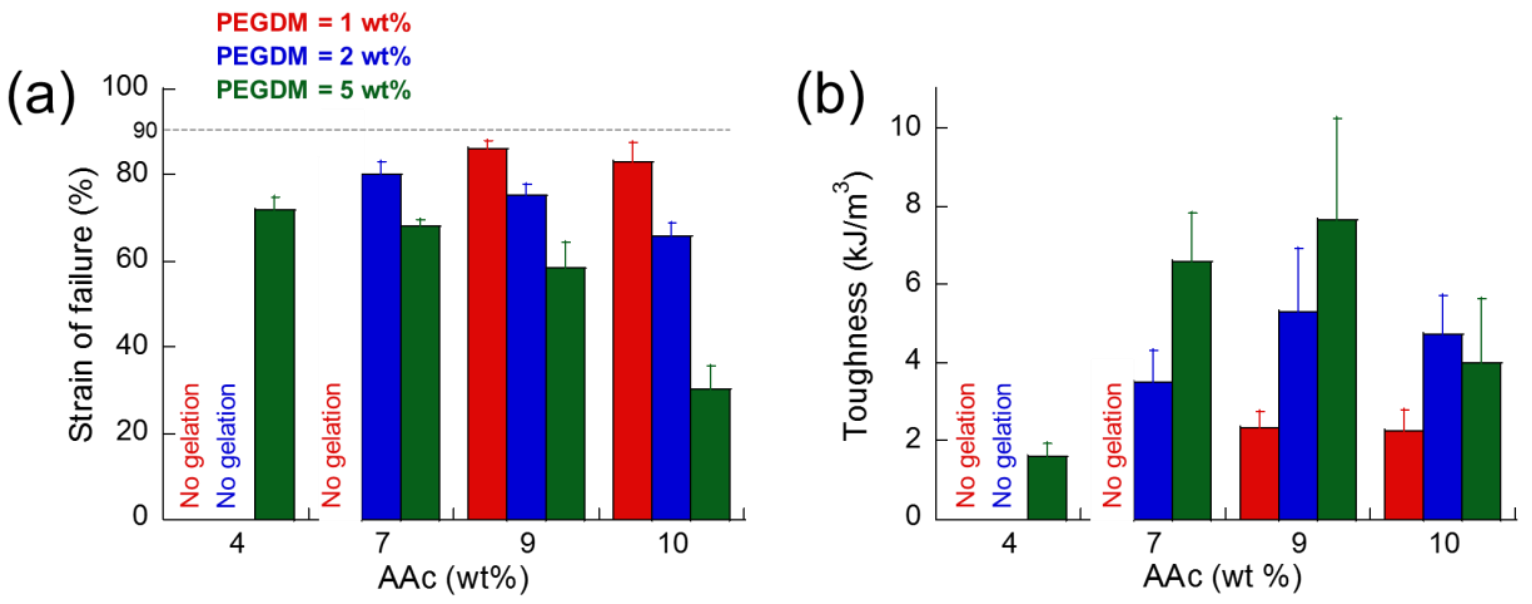

Figure A3. Many hydrogel formulations exhibit high strains of failure and toughness. Strain of failure (a) and toughness (b) vs. acrylic acid concentration for gels crosslinked with 1 wt\% (red), 2 wt\% (blue), and 5 wt\% (green) PEGDM. "No gelation" indicates that there was not enough polymer and crosslinker to form a 3D gel structure and therefore no measurement could be taken. For all parts, $\mathrm{N}=4$. In part (a) the dashed line at $90 \%$ indicates that compression tests never exceeded $90 \%$ as to not risk damaging the equipment, though no gels reached $90 \%$ compression due to failure at lower strains. 


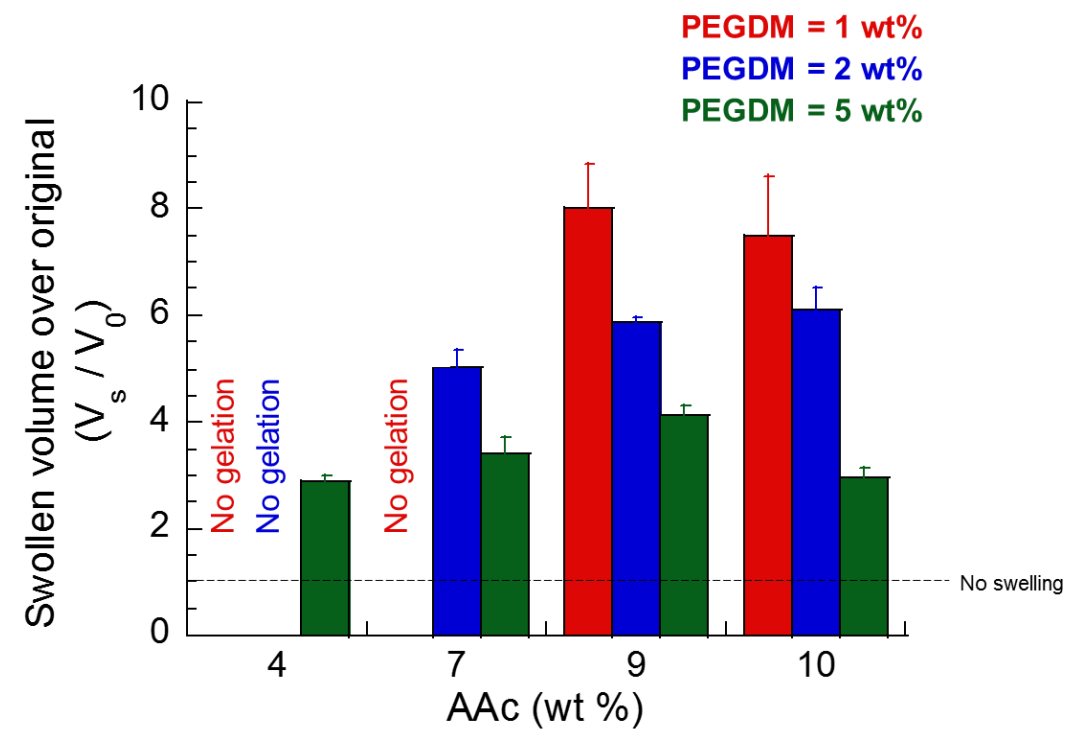

Figure A4. Nanoporous PEDOT/pAAc gel formulations swelled well beyond their original volumes after casting. Ratio of swollen volume over ordinally cast volume for PEDOT/pAAc gels vs. acrylic acid concentration for gels crosslinked with $1 \mathrm{wt} \%$ (red), $2 \mathrm{wt} \%$ (blue), and $5 \mathrm{wt} \%$ (green) PEGDM. "No gelation" indicates that there was not enough polymer and crosslinker to form a 3D gel structure and therefore no measurement could be taken. For all parts, $\mathrm{N}=4$. 


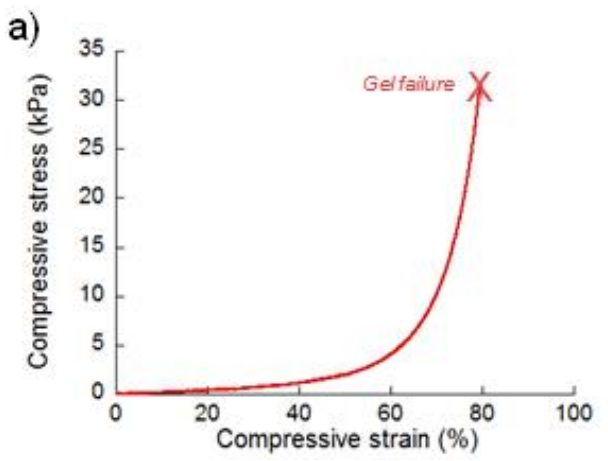

(b)

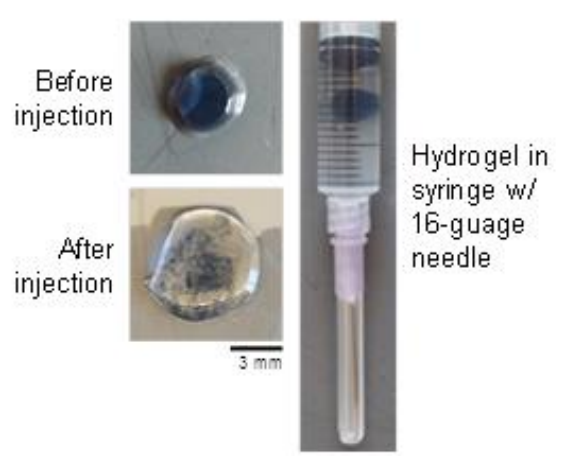

Figure A5. Nanoporous PEDOT/pAAc gel cannot survive the strains associated with injection. (a) Compressive stress vs. strain of for a 5 x $5 \mathrm{~cm}$ cylindrical $9 \mathrm{wt} \%$ AAc, 0.26 wt $\%$ PEDOT, $1 \mathrm{wt} \%$ PEGDM hydrogel, until failure (b) Photographs of a 3 x $1.25 \mathrm{~mm}$ cylindrical hydrogel (formulated as in part (a)) before and after injection through a 16-guage needle. 
(a)

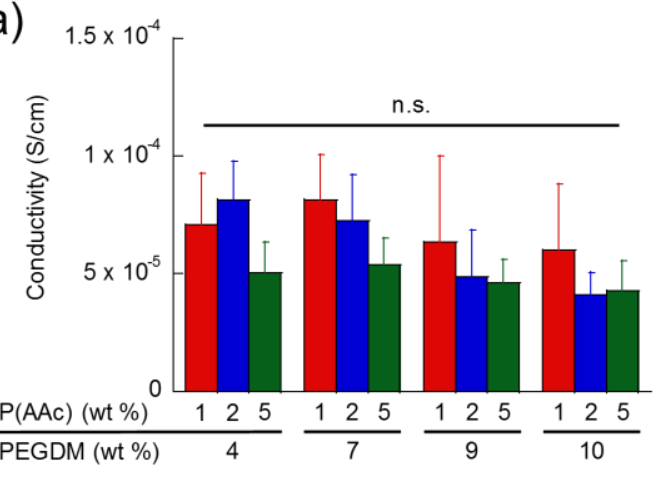

(c)

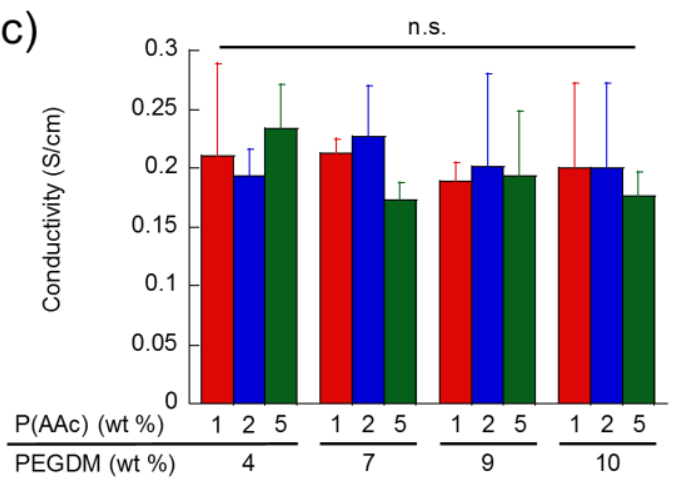

(b)

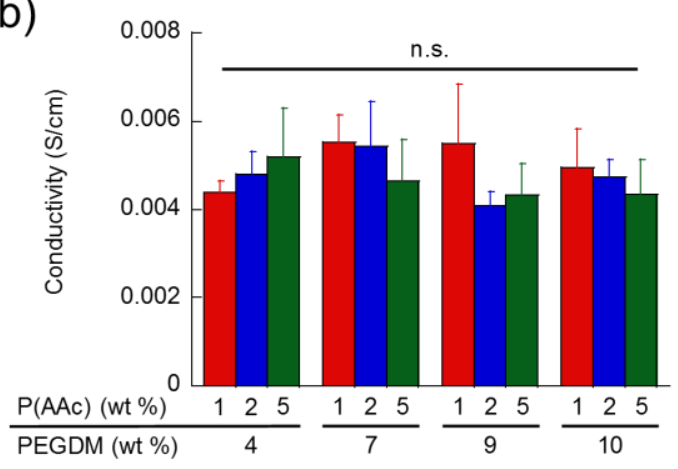

(d)

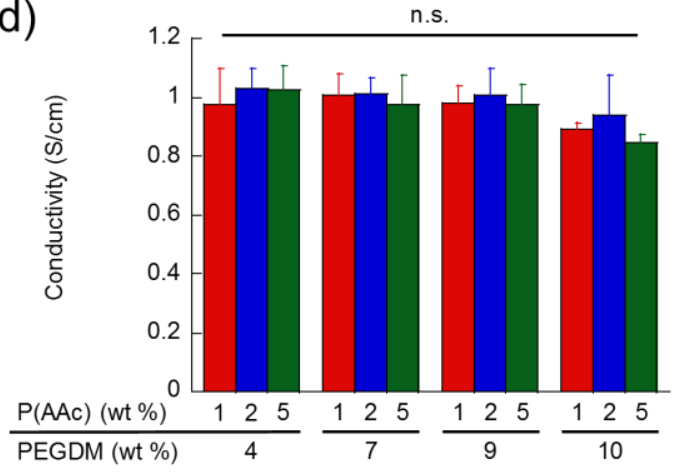

Figure A6. PEDOT cryogels exhibited similar DC conductivities over a wide range of gel formulations. DC conductivity vs. gel formulation for cryogels containing $0.26 \mathrm{wt} \%$ PEDOT when excited at $0.5 \mathrm{~V}(\mathrm{a}), 2 \mathrm{~V}(\mathrm{~b}), 4 \mathrm{~V}(\mathrm{c})$, and $8 \mathrm{~V}(\mathrm{~d})$. n.s. indicates that no statistically significant differences were found $(\mathrm{N}=4)$. 

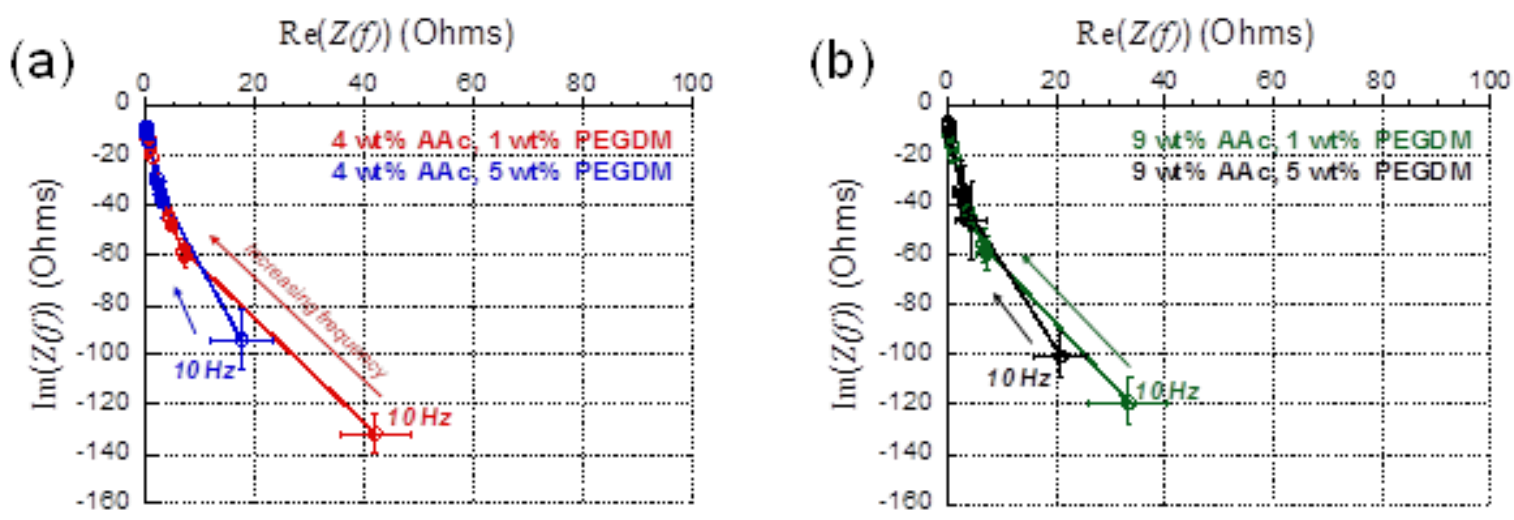

Figure A7. Nyquist plots for a range of PEDOT cryogel formulations exhibit similar characteristics. (a) Real vs. imaginary component of impedance for 4 wt $\%$ AAc PEDOT cryogels crosslinked with 1 wt $\%$ (red) and 5 wt $\%$ (blue) PEGDM. (b) Real vs. imaginary component of impedance for 9 wt $\%$ AAc PEDOT cryogels crosslinked with $1 \mathrm{wt} \%$ (green) and $5 \mathrm{wt} \%$ (black) PEGDM. $\mathrm{N}=4$. 


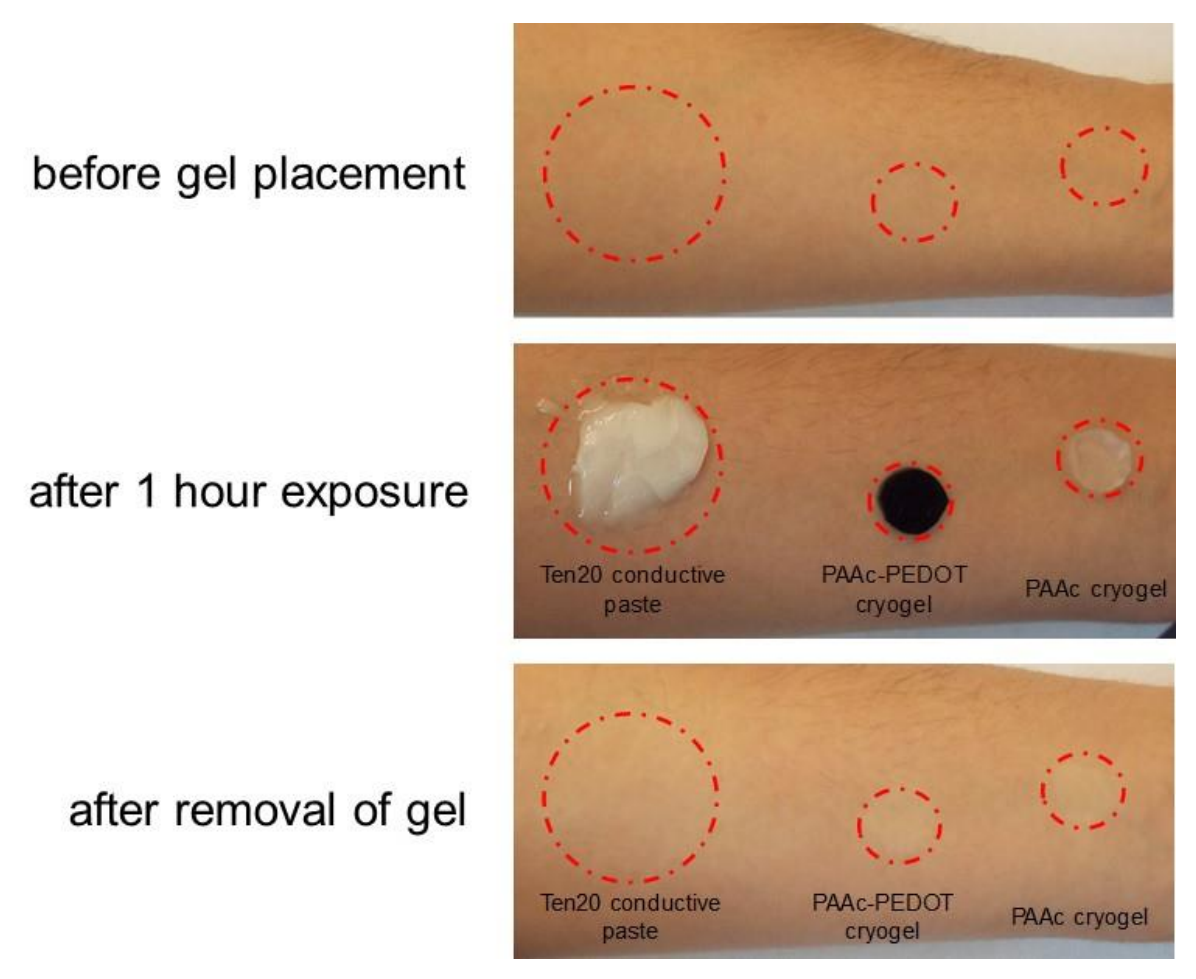

Figure A8. Exposure to PEDOT cryogels did not visually impact the skin. Subject forearm before gel placement (top), after 1 hour of exposure when gels (middle), and immediately after gel removal (bottom). PAAc-PEDOT cryogel was made at $9 \mathrm{wt} \%$ AAc, $1 \mathrm{wt} \%$ PEGDM, and $0.26 \mathrm{wt} \%$ PEDOT. The PAAc cryogel was made at $9 \mathrm{wt} \%$ AAc, $1 \mathrm{wt} \%$ PEGDM, and $0 \mathrm{wt} \%$ PEDOT. 
(a)

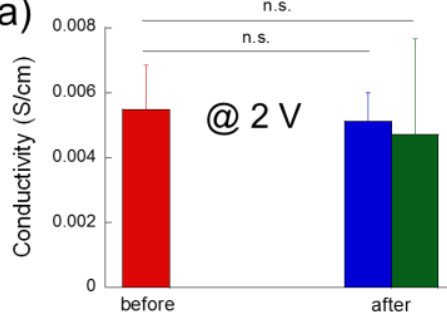

(b)

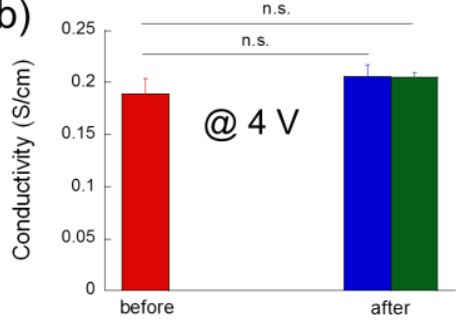

(c)

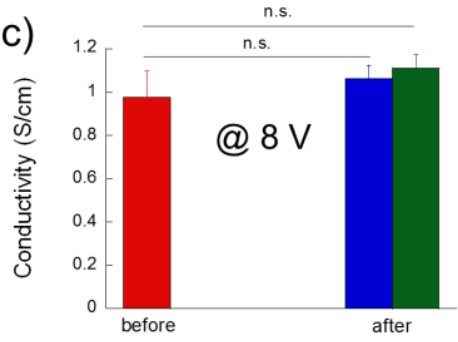

Figure A9. Ethanol-treatment and autoclaving PEDOT cryogels do not statistically alter electrical conductivity. DC electrical conductivity computed at $2 \mathrm{~V}$ (a), $4 \mathrm{~V}$ (b), and $8 \mathrm{~V}$ (c) for PEDOT cryogels (9 wt\% AAc, 1 wt\% PEGDM, and 0.26 wt\% PEDOT) before treatment (red), after ethanol-treatment (blue), or after autoclaving (green). n.s. indicates that no statistically significant differences were observed $(\mathrm{N}=4)$. 

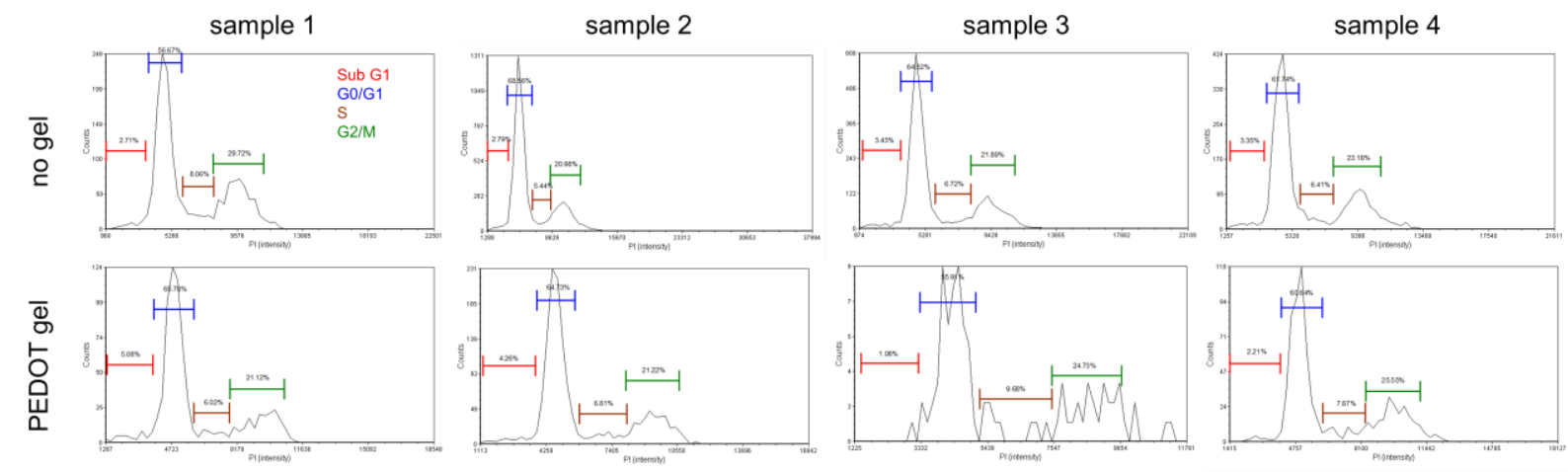

Figure A10. PEDOT cryogel-exposed fibroblasts do not exhibit different cell cycles as compared to controls. Histograms of propidium iodide (PI) intensity for fibroblasts after 10 days when not exposed to PEDOT cryogels (top row) compared to fibroblasts exposed to PEDOT cryogels (bottom row). 


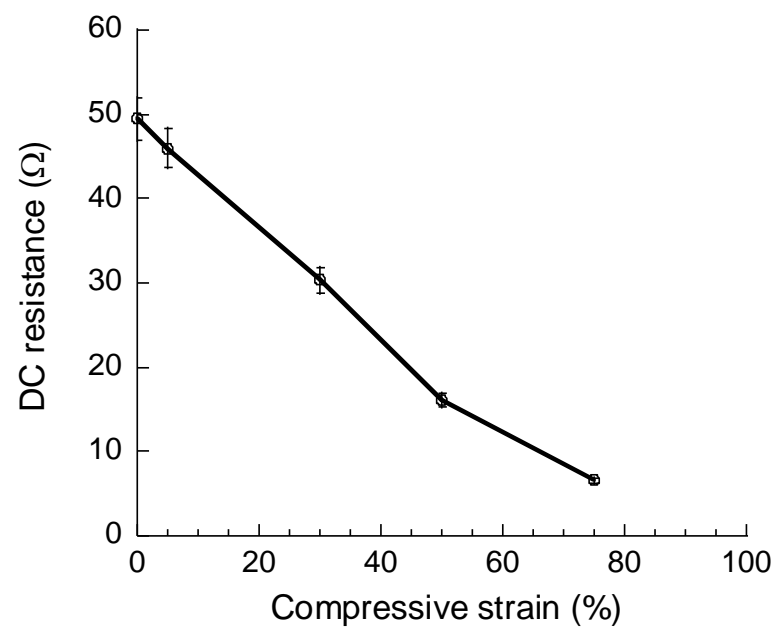

Figure A11. PEDOT/pAAc cryogel DC resistance decreases as the gel is compressed. DC resistance vs. compressive strain for $9 \mathrm{wt} \%$ AAc, $0.26 \mathrm{wt} \%$ PEDOT, $1 \mathrm{wt} \%$ PEGDM cryogels. $\mathrm{N}=4$. 


\section{APPENDIX B}

\section{Supplementary Materials for Chapter 4}
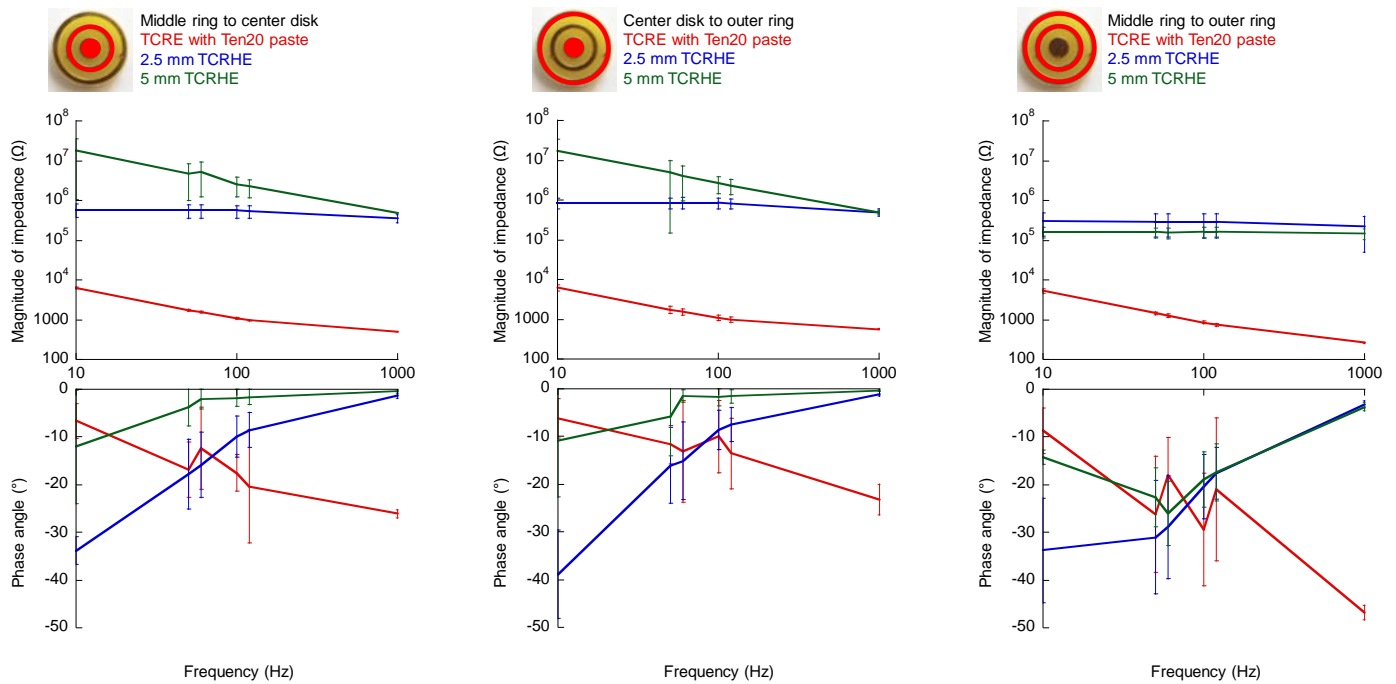

Figure B1. TCR hydrogel electrode have a much better isolation between channels. High impedance between channels indicates isolation. (A) isolation between middle ring to center disc. Red for TCRE with Ten20 paste, blue for $2.5 \mathrm{~mm}$ TCR hydrogel electrode (2.5 mm TCRHE) and green $5 \mathrm{~mm}$ TCR hydrogel electrode (5 mm TCRHE) (2 wt \% alginate, $2.5 \mathrm{mM}$ AAD and 0.26 wt \% PEDOT). isolation between center disc to outer ring. Red for TCRE with Ten20 paste, blue for $2.5 \mathrm{~mm}$ TCR hydrogel electrode (2.5 mm TCRHE) and green $5 \mathrm{~mm}$ TCR hydrogel electrode (5 mm TCRHE) ( $2 \mathrm{wt} \%$ alginate, $2.5 \mathrm{mM}$ AAD and $0.26 \mathrm{wt} \%$ PEDOT). (C) isolation between outer ring to middle ring. Red for TCRE with Ten20 paste, blue for $2.5 \mathrm{~mm}$ TCR hydrogel electrode (2.5 mm TCRHE) and green $5 \mathrm{~mm}$ TCR hydrogel 
electrode (5 mm TCRHE) (2 wt \% alginate, $2.5 \mathrm{mM}$ AAD and 0.26 wt \% PEDOT). $\mathrm{N}$

$=$ for all parts. 

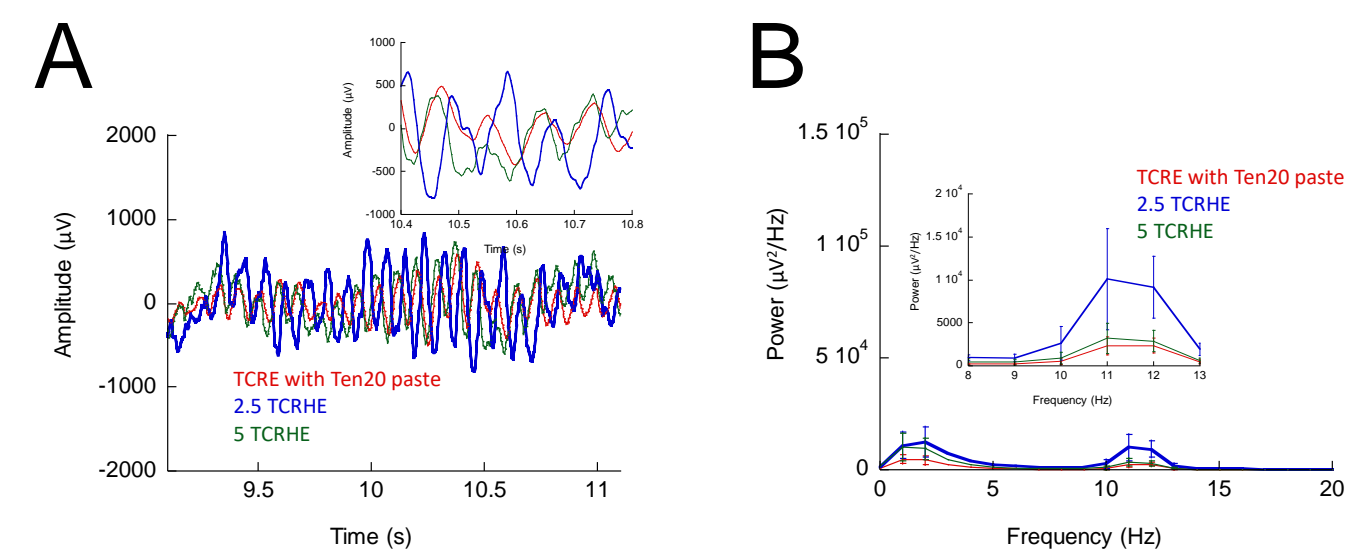

Eyes close

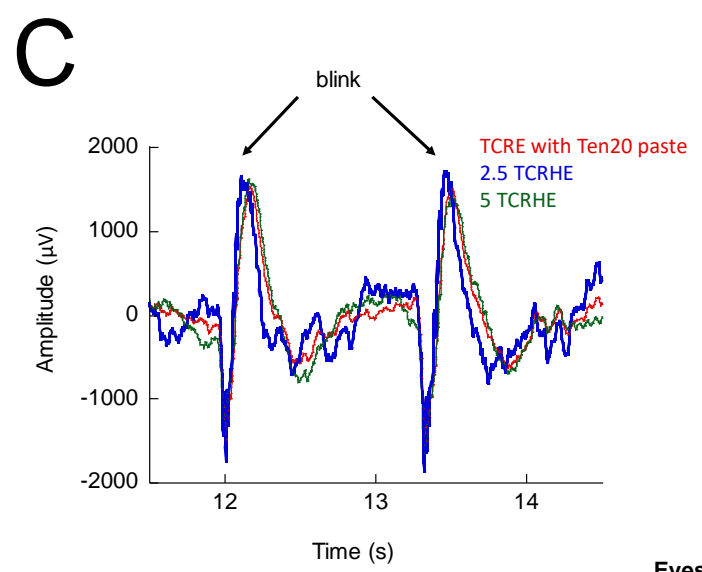

D

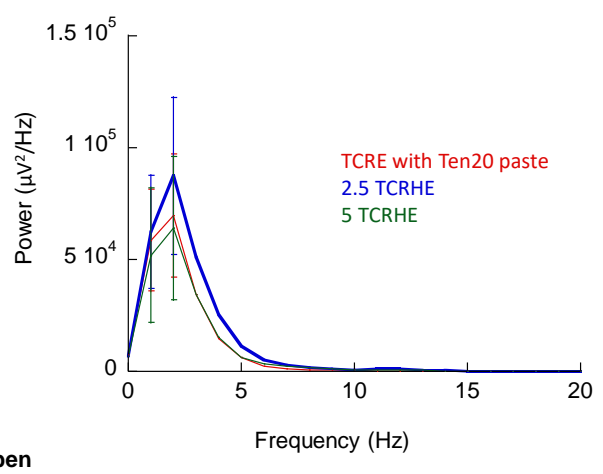

Figure B2. TCR hydrogel electrode have higher amplitude and significantly improves power spectrum comparing to TCRE with Ten20 paste. (A) EEG recording from occipital lobe using TEN20 paste (red) compared to hydrogel (2.5 mm lead electrode (blue) and $5 \mathrm{~mm}$ lead electrode) while subject eyes were closed. (i) Inset: zoom-in EEG recording while eyes were closed. (B) Power spectrum from occipital lobe using TEN20 paste (red) compared to hydrogel $(2.5 \mathrm{~mm}$ electrode lead (blue) and $5 \mathrm{~mm}$ lead electrode (green)) while subject eyes were closed. (i) Inset: zoom-in power spectra, highlighting the recording of alpha waves $(8-13 \mathrm{~Hz})$ while eyes were closed. (C) EEG recording from occipital lobe using TEN20 paste (red) compared to hydrogel ( $2.5 \mathrm{~mm}$ lead electrode (blue) and $5 \mathrm{~mm}$ lead electrode) while 
subject eyes were opened. Alfa wave was gone as soon as eyes were opened. (D) Power spectrum from occipital lobe using TEN20 paste (red) compared to hydrogel (2.5 mm lead electrode (blue) and $5 \mathrm{~mm}$ lead electrode(green)) while subject eyes were open. Alfa wave was gone as soon as eyes were opened. For all parts, $\mathrm{N}=4$ at 0 hour. 


\section{BIBLIOGRAPHY}

(2013). Handbook of Clinical Neurology. Amesterdam, Netherlands, Elsevier, B. V.

Abidian, M. R., et al. (2006). "Conducting-Polymer Nanotubes for Controlled Drug Release." Adv Mater 18(4): 405-409.

Abidian, M. R. and D. C. Martin (2008). "Experimental and theoretical characterization of implantable neural microelectrodes modified with conducting polymer nanotubes." Biomaterials 29(9): 1273-1283.

Abidian, M. R. and D. C. Martin (2009). "Multifunctional Nanobiomaterials for Neural Interfaces." Advanced Functional Materials 19(4): 573-585.

Alam, M., et al. (2016). "Brain-machine interface facilitated neurorehabilitation via spinal stimulation after spinal cord injury: Recent progress and future perspectives." Brain Res 1646: 25-33.

Antensteiner, M., et al. (2017). "Conducting Polymer Microcups for Organic Bioelectronics and Drug Delivery Applications." Advanced Materials 29(39): 1702576.

Aregueta-Robles, U. A., et al. (2014). "Organic electrode coatings for next-generation neural interfaces." frontiers in Neuroengineering 7(15).

Asplund, M., et al. (2010). "Electroactive polymers for neural interfaces." Polymer Chemistry 1(9).

Audu, M. L., et al. (2015). "A neuroprosthesis for control of seated balance after spinal cord injury." Journal of NeuroEngineering and Rehabilitation 12(1): 8.

Balint, R., et al. (2014). "Conductive polymers: towards a smart biomaterial for tissue engineering." Acta Biomater 10(6): 2341-2353. 
Benbadis, S. R., et al. (2004). "Outcome of prolonged video-EEG monitoring at a typical referral epilepsy center." Epilepsia 45(9): 1150-1153.

Bencherif, S. A., et al. (2012). "Injectable preformed scaffolds with shape-memory properties." Proc Natl Acad Sci U S A 109(48): 19590-19595.

Bergey, G. K. (2013). "Neurostimulation in the treatment of epilepsy." Exp Neurol 244: 87-95.

Besio, W., et al. (2006). "Development of a Tri-polar Concentric Ring Electrode for Acquiring Accurate Laplacian Body Surface Potentials." Annals of Biomedical Engineering 34(3): 426-435.

Besio, W. and T. Chen (2007). "Tripolar Laplacian electrocardiogram and moment of activation isochronal mapping." Physiological Measurement 28(5): 515-529.

Besio, W. G., et al. (2006). "Tri-polar concentric ring electrode development for laplacian electroencephalography." IEEE Trans Biomed Eng 53(5): 926-933.

Besio, W. G., et al. (2001). "A feasibility study for body surface cardiac propagation maps of humans from Laplacian moments of activation." Electromagnetics 21(7-8): 621-632.

Bhat, S. and A. Kumar (2013). "Biomaterials and bioengineering tomorrow's healthcare." Biomatter 3(3).

Bianchi, G., et al. (2016). "Electrochemotherapy in the Treatment of Bone Metastases: A Phase II Trial." World J Surg 40(12): 3088-3094.

Bjordal, J. M., et al. (2003). "Transcutaneous electrical nerve stimulation (TENS) can reduce postoperative analgesic consumption. A meta-analysis with assessment 
of optimal treatment parameters for postoperative pain." European Journal of Pain 7(2): 181-188.

Brudno, Y. and D. J. Mooney (2015). "On-demand drug delivery from local depots." J Control Release 219: 8-17.

Brunoni, A. R., et al. (2012). "Clinical research with transcranial direct current stimulation (tDCS): challenges and future directions." Brain Stimul 5(3): 175195.

Calder, S., et al. (2017). Anatomical variations of the stomach effects on electrogastrography. Engineering in Medicine and Biology Society (EMBC), 2017 39th Annual International Conference of the IEEE, Seogwipo, South Korea, IEEE.

Carmel, J. B., et al. (2013). "Motor cortex electrical stimulation promotes axon outgrowth to brain stem and spinal targets that control the forelimb impaired by unilateral corticospinal injury." Eur J Neurosci 37(7): 1090-1102.

Carvalhaes, C. and J. A. de Barros (2015). "The surface Laplacian technique in EEG: Theory and methods." Int J Psychophysiol 97(3): 174-188.

Cellot, G., et al. (2015). "PEDOT:PSS Interfaces Support the Development of Neuronal Synaptic Networks with Reduced Neuroglia Response In vitro." Front Neurosci 9: 521.

Cezar, C. A., et al. (2014). "Biphasic ferrogels for triggered drug and cell delivery." Adv Healthc Mater 3(11): 1869-1876.

Chang, C., et al. (2010). "Superabsorbent hydrogels based on cellulose for smart swelling and controllable delivery." European Polymer Journal 46(1): 92-100. 
Chang, P., et al. (2013). "Seizure control by ketogenic diet-associated medium chain fatty acids." Neuropharmacology 69: 105-114.

Chansai, P., et al. (2009). "Controlled transdermal iontophoresis of sulfosalicylic acid from polypyrrole/poly(acrylic acid) hydrogel." Int J Pharm 381(1): 25-33.

Chen, C., et al. (2017). "Electrically-responsive core-shell hybrid microfibers for controlled drug release and cell culture." Acta Biomater 55: 434-442.

Chen, M., et al. (2014). "Electrospun nanofibers-mediated on-demand drug release." Adv Healthc Mater 3(11): 1721-1732.

Chen, Y. H., et al. (2014). "Soft, comfortable polymer dry electrodes for high quality ECG and EEG recording." Sensors (Basel) 14(12): 23758-23780.

Chikar, J. A., et al. (2012). "The use of a dual PEDOT and RGD-functionalized alginate hydrogel coating to provide sustained drug delivery and improved cochlear implant function." Biomaterials 33(7): 1982-1990.

Cho, W., et al. (2015). "Synthesis and characterization of bicontinuous cubic poly(3,4ethylene dioxythiophene) gyroid (PEDOT GYR) gels." Phys Chem Chem Phys 17(7): 5115-5123.

Choi, E. J., et al. (2015). "Synthesis of electroconductive hydrogel films by an electrocontrolled click reaction and their application to drug delivery systems." Polymer Chemistry 6(24): 4473-4478.

Choi, Y. D. and J. H. Lee (2016). "Edema and pain reduction using transcutaneous electrical nerve stimulation treatment." J Phys Ther Sci 28(11): 3084-3087.

Coffman, B. A., et al. (2017). "Reduced auditory segmentation potentials in firstepisode schizophrenia." Schizophr Res. 
Cogan, S. F. (2008). "Neural stimulation and recording electrodes." Annu Rev Biomed Eng 10: 275-309.

Da Cunha, C., et al. (2015). "Toward sophisticated basal ganglia neuromodulation: Review on basal ganglia deep brain stimulation." Neurosci Biobehav Rev 58: 186-210.

Dai, T., et al. (2009). "Conducting hydrogels with enhanced mechanical strength." Polymer 50(22): 5236-5241.

Dai, T., et al. (2010). "Mechanically strong conducting hydrogels with special doublenetwork structure." Synthetic Metals 160(7-8): 791-796.

Dai, T., et al. (2010). "Self-strengthened conducting polymer hydrogels." Synthetic Metals 160(9-10): 1101-1106.

de Guzman, R. C. (2018). "PABA Release from Chitosan-PCL with Induced Electric Current." Biomedical Journal of Scientific \& Technical Research 7(3).

Dee, K. C., et al. (2003). An itroduction to tissue-biomaterial interactions. Hoboken, John Wiley \&Sons.

Dubey, S. and Y. N. Kalia (2014). "Understanding the poor iontophoretic transport of lysozyme across the skin: when high charge and high electrophoretic mobility are not enough." J Control Release 183: 35-42.

Edhemovic, I., et al. (2011). "Electrochemotherapy: a new technological approach in treatment of metastases in the liver." Technol Cancer Res Treat 10(5): 475485.

Edlow, B. L., et al. (2017). "Early detection of consciousness in patients with acute severe traumatic brain injury." Brain 140(9): 2399-2414. 
Engler, A. J., et al. (2006). "Matrix elasticity directs stem cell lineage specification." Cell 126(4): 677-689.

Erturk, G. and B. Mattiasson (2014). "Cryogels-versatile tools in bioseparation." J Chromatogr A 1357: 24-35.

Fan, Z., et al. (2016). "Significant Enhancement in the Thermoelectric Properties of PEDOT:PSS Films through a Treatment with Organic Solutions of Inorganic Salts." ACS Appl Mater Interfaces 8(35): 23204-23211.

Fantozzi, F., et al. (2010). "The effects of the electric fields on hydrogels to achieve antitumoral drug release." Bioelectrochemistry 78(2): 191-195.

Farina, D. and C. Cescon (2001). "Concentric-ring electrode systems for noninvasive detection of single motor unit activity." IEEE Trans Biomed Eng 48(11): 13261334.

Farra, R., et al. (2012). "First-in-human testing of a wirelessly controlled drug delivery microchip." Sci Transl Med 4(122): 122ra121.

Fattahi, P., et al. (2014). "A review of organic and inorganic biomaterials for neural interfaces." Adv Mater 26(12): 1846-1885.

Feig, V. R., et al. (2018). "Mechanically tunable conductive interpenetrating network hydrogels that mimic the elastic moduli of biological tissue." Nat Commun 9(1): 2740 .

Ferlauto, L., et al. (2018). "Development and Characterization of PEDOT:PSS/Alginate Soft Microelectrodes for Application in Neuroprosthetics." Front Neurosci 12: 648. 
Feron, K., et al. (2018). "Organic Bioelectronics: Materials and Biocompatibility." Int J Mol Sci 19(8).

Filipcsei, G., et al. (2000). "Electric field sensitive neutral polymer gels." Journal of Molecular Structure 554(1): 109-117.

Fridman, G. Y. and C. C. Della Santina (2012). "Progress toward development of a multichannel vestibular prosthesis for treatment of bilateral vestibular deficiency." Anat Rec (Hoboken) 295(11): 2010-2029.

Gabriel, S., et al. (1996). "The dielectric properties of biological tissues: II. Measurements in the frequency range $10 \mathrm{~Hz}$ to $20 \mathrm{GHz} . "$ Phys Med Biol 41(11): 2251-2269.

Ganguly, S., et al. (2018). "Green Reduced Graphene Oxide Toughened Semi-IPN Monolith Hydrogel as Dual Responsive Drug Release System: Rheological, Physicomechanical, and Electrical Evaluations." J Phys Chem B 122(29): 7201-7218.

Ganguly, S., et al. (2018). "Mechanically robust dual responsive water dispersiblegraphene based conductive elastomeric hydrogel for tunable pulsatile drug release." Ultrason Sonochem 42: 212-227.

Gao, L. X., et al. (2015). "Electro-response characteristic of starch hydrogel crosslinked with Glutaraldehyde." J Biomater Sci Polym Ed 26(9): 545-557.

Gasselhuber, A., et al. (2010). "Mathematical spatio-temporal model of drug delivery from low temperature sensitive liposomes during radiofrequency tumour ablation." Int J Hyperthermia 26(5): 499-513. 
Ge, J., et al. (2012). "Drug release from electric-field-responsive nanoparticles." ACS Nano 6(1): 227-233.

Ghasemi-Mobarakeh, L., et al. (2011). "Application of conductive polymers, scaffolds and electrical stimulation for nerve tissue engineering." J Tissue Eng Regen Med 5(4): e17-35.

Green, R. A., et al. (2012). "Variation in Performance of Platinum Electrodes with Size and Surface Roughness." sensors and materials 24(4): 165-180.

Green, R. A., et al. (2008). "Novel neural interface for implant electrodes: improving electroactivity of polypyrrole through MWNT incorporation." J Mater Sci Mater Med 19(4): 1625-1629.

Guarino, V., et al. (2016). "Electro-Active Polymers (EAPs): A Promising Route to Design Bio-Organic/Bioinspired Platforms with on Demand Functionalities." Polymers 8(5).

Guillet, J. F., et al. (2017). "A Hydrogel/Carbon-Nanotube Needle-Free Device for Electrostimulated Skin Drug Delivery." Chemphyschem 18(19): 2715-2723.

Guimard, N. K., et al. (2007). "Conducting polymers in biomedical engineering." Progress in Polymer Science 32(8-9): 876-921.

Guiseppi-Elie, A. (2010). "Electroconductive hydrogels: synthesis, characterization and biomedical applications." Biomaterials 31(10): 2701-2716.

Gulyuz, U. and O. Okay (2014). "Self-Healing Poly(acrylic acid) Hydrogels with Shape Memory Behavior of High Mechanical Strength." Macromolecules 47(19): 6889-6899. 
Gun'ko, V. M., et al. (2013). "Cryogels: morphological, structural and adsorption characterisation." Adv Colloid Interface Sci 187-188: 1-46.

Guo, B., et al. (2013). "Biodegradable and electrically conducting polymers for biomedical applications." Progress in Polymer Science 38(9): 1263-1286.

Hammond, C., et al. (2008). "Latest view on the mechanism of action of deep brain stimulation." Mov Disord 23(15): 2111-2121.

Hassarati, R. T., et al. (2014). "Improving cochlear implant properties through conductive hydrogel coatings." IEEE Trans Neural Syst Rehabil Eng 22(2): 411-418.

He, B. and R. J. Cohen (1992). "Body surface Laplacian ECG mapping." IEEE Trans Biomed Eng 39(11): 1179-1191.

He, B., et al. (2001). "High-resolution EEG: a new realistic geometry spline Laplacian estimation technique." Clinical Neurophysiology 112(5): 845-852.

Herrington, T. M., et al. (2016). "Mechanisms of deep brain stimulation." J Neurophysiol 115(1): 19-38.

Hollis, S. and P. McClure (2017). "Intramuscular Electrical Stimulation for Muscle Activation of the Tibialis Anterior After Surgical Repair: A Case Report." J Orthop Sports Phys Ther 47(12): 965-969.

Horvath, A., et al. (2018). "EEG and ERP biomarkers of Alzheimer's disease: a critical review." Front Biosci (Landmark Ed) 23: 183-220.

Hosseini-Nassab, N., et al. (2017). "Electrically controlled release of insulin using polypyrrole nanoparticles." Nanoscale 9(1): 143-149. 
Hosseinzadeh, S., et al. (2016). "Nanofibrous hydrogel with stable electrical conductivity for biological applications." Polymer 97: 205-216.

Hsu, C. S. and L. H. Block (1996). "Anionic gels as vehicles for electricallymodulated drug delivery. solvent and drug transport phenomena." Pharmaceutical Research 13(12): 1865-1870.

Huang, P., et al. (2013). "An inorganic-organic double network hydrogel of graphene and polymer." Nanoscale 5(13): 6034-6039.

Huang, W.-C., et al. (2011). "Characterization and drug release behavior of chip-like amphiphilic chitosan-silica hybrid hydrogel for electrically modulated release of ethosuximide: an in vitro study." Journal of Materials Chemistry 21(40).

Hur, J., et al. (2014). "Polypyrrole/Agarose-based electronically conductive and reversibly restorable hydrogel." ACS Nano 8(10): 10066-10076.

Hutten, M., et al. (2014). "In vitro and in vivo evaluation of a hydrogel reservoir as a continuous drug delivery system for inner ear treatment." PLoS One 9(8): e104564.

Im, J. S., et al. (2010). "The effect of carbon nanotubes on drug delivery in an electrosensitive transdermal drug delivery system." Biomaterials 31(6): 1414-1419.

Inal, S., et al. (2018). "Conjugated Polymers in Bioelectronics." Acc Chem Res 51(6): 1368-1376.

Indermun, S., et al. (2014). "An interfacially plasticized electro-responsive hydrogel for transdermal electro-activated and modulated (TEAM) drug delivery." Int J Pharm 462(1-2): 52-65. 
Indermun, S., et al. (2014). "Patient-controlled analgesia: therapeutic interventions using transdermal electro-activated and electro-modulated drug delivery." J Pharm Sci 103(2): 353-366.

Isseroff, R. R. and S. E. Dahle (2012). "Electrical Stimulation Therapy and Wound Healing: Where Are We Now?" Adv Wound Care (New Rochelle) 1(6): 238243.

Javadi, M., et al. (2018). "Conductive Tough Hydrogel for Bioapplications." Macromol Biosci 18(2).

Jensen, M., et al. (2002). "Loading into and electro-stimulated release of peptides and proteins from chondroitin 4-sulphate hydrogels." European Journal of Pharmaceutical Sciences 15(2): 139-148.

Jeon, G., et al. (2011). "Electrically actuatable smart nanoporous membrane for pulsatile drug release." Nano Lett 11(3): 1284-1288.

Jin, X., et al. (2008). "Synthesis and properties of electrically-sensitive poly(acrylic acid-co-acetoacetoxy ethyl methacrylate) gels." Journal of Applied Polymer Science 110(6): 3690-3696.

Jo, H., et al. (2017). "Electrically conductive graphene/polyacrylamide hydrogels produced by mild chemical reduction for enhanced myoblast growth and differentiation." Acta Biomater 48: 100-109.

Juntanon, K., et al. (2008). "Electrically controlled release of sulfosalicylic acid from crosslinked poly(vinyl alcohol) hydrogel." Int J Pharm 356(1-2): 1-11. 
Kantaria, S., et al. (1999). "Gelatin-stabilised microemulsion-based organogels: rheology and application in iontophoretic transdermal drug delivery." Journal of Controlled Release 60(2-3): 355-365.

Kapural, L., et al. (2005). "Occipital nerve electrical stimulation via the midline approach and subcutaneous surgical leads for treatment of severe occipital neuralgia: a pilot study." Anesth Analg 101(1): 171-174, table of contents.

Kearney, C. J. and D. J. Mooney (2013). "Macroscale delivery systems for molecular and cellular payloads." Nat Mater 12(11): 1004-1017.

Kennedy, S., et al. (2014). "Rapid and extensive collapse from electrically responsive macroporous hydrogels." Adv Healthc Mater 3(4): 500-507.

Khorrami, M. and M. R. Abidian (2018). "Aligned Conducting Polymer Nanotubes for Neural Prostheses." Conf Proc IEEE Eng Med Biol Soc 2018: 6080-6083.

Kiaee, G., et al. (2018). "A pH-Mediated Electronic Wound Dressing for Controlled Drug Delivery." Adv Healthc Mater: e1800396.

Kilby, J., et al. (2016). Design of new multi-channel electrodes for surface electromyography signals for signal-processing. IEEE 38th annual international conference of Engineering in Medicine and Biology Society (EMBC), Orlando, FL, USA, IEEE.

Kim, D. H., et al. (2004). "Conducting polymers grown in hydrogel scaffolds coated on neural prosthetic devices." J Biomed Mater Res A 71(4): 577-585.

Kim, S. and K. E. Healy (2003). "Synthesis and Characterization of Injectable Poly(Nisopropylacrylamide-co-acrylic acid) Hydrogels with Proteolytically Degradable Cross-Links." Biomacromolecules 4(5): 1214-1223. 
Kim, S. J., et al. (2003). "Characteristics of electrical responsive alginate/poly(diallyldimethylammonium chloride) IPN hydrogel in $\mathrm{HCl}$ solutions." Sensors and Actuators B: Chemical 96(1-2): 1-5.

Kim, S. Y. and Y. M. Lee (1999). "Drug release behavior of electrical responsive poly(vinyl alcohol)/poly(acrylic acid) IPN hydrogels under an electric stimulus." Journal of Applied Polymer Science 74(7): 1752-1761.

Kitamura, T., et al. (2018). "Control with Adjusted Pulse Frequency and Amplitude in Functional Electrical Stimulation." Conf Proc IEEE Eng Med Biol Soc 2018: 2064-2067.

Kleber, C., et al. (2017). "An interpenetrating, microstructurable and covalently attached conducting polymer hydrogel for neural interfaces." Acta Biomater 58: 365-375.

Koetting, M. C., et al. (2015). "Stimulus-responsive hydrogels: Theory, modern advances, and applications." Mater Sci Eng R Rep 93: 1-49.

Koka, K. and W. G. Besio (2007). "Improvement of spatial selectivity and decrease of mutual information of tri-polar concentric ring electrodes." J Neurosci Methods 165(2): 216-222.

Koutsioumpa, E., et al. (2018). "Effect of Transcutaneous Electrical Neuromuscular Stimulation on Myopathy in Intensive Care Patients." Am J Crit Care 27(6): 495-503.

Kozai, T. D., et al. (2015). "Brain tissue responses to neural implants impact signal sensitivity and intervention strategies." ACS Chem Neurosci 6(1): 48-67. 
Kringelbach, M. L., et al. (2007). "Translational principles of deep brain stimulation." Nat Rev Neurosci 8(8): 623-635.

Krukiewicz, K., et al. (2016). "An electrically controlled drug delivery system based on conducting poly(3,4-ethylenedioxypyrrole) matrix." Bioelectrochemistry 108: $13-20$.

Kulkarni, R. V. and B. Sa (2009). "Electroresponsive Polyacrylamide-grafted-xanthan Hydrogels for Drug Delivery." Journal of Bioactive and Compatible Polymers 24(4): 368-384.

Kulkarni, R. V., et al. (2010). "Polyacrylamide-g-alginate-based electrically responsive hydrogel for drug delivery application: Synthesis, characterization, and formulation development." Journal of Applied Polymer Science 115(2): 1180-1188.

Kulsh, J. (1997). "Targeting a key enzyme in cell growth: a novel therapy for cancer." Medical Hypotheses 49(4): 297-300.

Kumar, A. and A. Srivastava (2010). "Cell separation using cryogel-based affinity chromatography." Nat Protoc 5(11): 1737-1747.

Kumar, V. A., et al. (2015). "Self-assembling multidomain peptides tailor biological responses through biphasic release." Biomaterials 52: 71-78.

Kuo, J. R., et al. (2015). "Deep brain light stimulation effects on glutamate and dopamine concentration." Biomed Opt Express 6(1): 23-31.

Kwon, I. C., et al. (1991). "Electrically erodible polymer gel for controlled release of drugs." Nature 354(6351): 291-293. 
Law, S. K., et al. (1993). "High-resolution EEG using spline generated surface Laplacians on spherical and ellipsoidal surfaces." IEEE Trans Biomed Eng 40(2): 145-153.

Leach, J., et al. (2010). "Bridging the divide between neuroprosthetic design, tissue engineering and neurobiology." frontiers in Neuroengineering 2(18).

Lee, S., et al. (2007). "Skin Permeation Enhancement of Ascorbyl Palmitate by Liposomal Hydrogel (Lipogel) Formulation and Electrical Assistance." Biological \& Pharmaceutical Bulletin 30(2): 393-396.

Lepley, L. K., et al. (2015). "Combination of eccentric exercise and neuromuscular electrical stimulation to improve quadriceps function post-ACL reconstruction." Knee 22(3): 270-277.

Li, G., et al. (2017). "Towards gel-free electrodes: A systematic study of electrodeskin impedance." Sensors and Actuators B: Chemical 241: 1244-1255.

Li, H. (2009). "Kinetics of smart hydrogels responding to electric field: A transient deformation analysis." International Journal of Solids and Structures 46(6): 1326-1333.

Li, L., et al. (2014). "In situ forming biodegradable electroactive hydrogels." Polymer Chemistry 5(8).

Li, L., et al. (2015). "Injectable conducting interpenetrating polymer network hydrogels from gelatin-graft-polyaniline and oxidized dextran with enhanced mechanical properties." RSC Advances 5(112): 92490-92498. 
Li, W., et al. (2015). "Polyaniline-poly(styrene sulfonate) conducting hydrogels reinforced by supramolecular nanofibers and used as drug carriers with electric-driven release." European Polymer Journal 66: 513-519.

Liang, Y., et al. (2019). "Adhesive Hemostatic Conducting Injectable Composite Hydrogels with Sustained Drug Release and Photothermal Antibacterial Activity to Promote Full-Thickness Skin Regeneration During Wound Healing." Small: e1900046.

Lin, J., et al. (2009). "Electric field sensitivity of conducting hydrogels with interpenetrating polymer network structure." Colloids and Surfaces A: Physicochemical and Engineering Aspects 346(1-3): 177-183.

Lira, L. M. and S. I. Córdoba de Torresi (2005). "Conducting polymer-hydrogel composites for electrochemical release devices: Synthesis and characterization of semi-interpenetrating polyaniline-polyacrylamide networks." Electrochemistry Communications 7(7): 717-723.

Liu, G. and X. Zhao (2007). "Electroresponsive Behavior of Gelatin/Alginate Semi-interpenetrating Polymer Network Membranes Under Direct-Current Electric Field." Journal of Macromolecular Science, Part A 43(2): 345-354.

Liu, K. H., et al. (2008). "Drug release behavior of chitosan-montmorillonite nanocomposite hydrogels following electrostimulation." Acta Biomater 4(4): 1038-1045.

Liu, Y., et al. (2012). "An electric-field responsive microsystem for controllable miniaturised drug delivery applications." Sensors and Actuators B: Chemical 175: 100-105. 
Lopez-Gordo, M. A., et al. (2014). "Dry EEG electrodes." Sensors (Basel) 14(7): 12847-12870.

Lu, C.-C. and P. P. Tarjan (2002). "Pasteless, Active, Concentric Ring Sensors for Directly Obtained Laplacian Cardiac Electrograms." Journal of Medical and Biological Engineering 22(4): 199-203.

Lu, Y., et al. (2014). "Elastic, conductive, polymeric hydrogels and sponges." Sci Rep 4: 5792 .

Luo, S. C., et al. (2008). "Poly(3,4-ethylenedioxythiophene) (PEDOT) nanobiointerfaces: thin, ultrasmooth, and functionalized PEDOT films with in vitro and in vivo biocompatibility." Langmuir 24(15): 8071-8077.

Luo, X. and X. T. Cui (2009). "Sponge-like nanostructured conducting polymers for electrically controlled drug release." Electrochem commun 11(10): 1956.

Lyons, M. K. (2011). "Deep brain stimulation: current and future clinical applications." Mayo Clin Proc 86(7): 662-672.

Mac Kenna, N., et al. (2015). "Electro-stimulated release from a reduced graphene oxide composite hydrogel." Journal of Materials Chemistry B 3(12): 25302537.

Maloney, J. M., et al. (2005). "Electrothermally activated microchips for implantable drug delivery and biosensing." J Control Release 109(1-3): 244-255.

Man, I. O., et al. (2007). "Effect of neuromuscular electrical stimulation on ankle swelling in the early period after ankle sprain." Phys Ther 87(1): 53-65. 
Manola, L., et al. (2005). "Modelling motor cortex stimulation for chronic pain control: electrical potential field, activating functions and responses of simple nerve fibre models." Med Biol Eng Comput 43(3): 335-343.

Mario Cheong, G. L., et al. (2014). "Conductive hydrogels with tailored bioactivity for implantable electrode coatings." Acta Biomater 10(3): 1216-1226.

Martin, D. C., et al. (2010). "The Morphology of Poly(3,4-Ethylenedioxythiophene)." Polymer Reviews 50(3): 340-384.

Marty, M., et al. (2006). "Electrochemotherapy - An easy, highly effective and safe treatment of cutaneous and subcutaneous metastases: Results of ESOPE (European Standard Operating Procedures of Electrochemotherapy) study." 4(11): 3-13.

Mawad, D., et al. (2016). Conductive Polymer Hydrogels. Polymeric Hydrogels as Smart Biomaterials. S. Kalia. Cham, Springer International Publishing: 19-44.

McFarland, D. J. (2015). "The advantages of the surface Laplacian in brain-computer interface research." Int J Psychophysiol 97(3): 271-276.

Merrill, D. R., et al. (2005). "Electrical stimulation of excitable tissue: design of efficacious and safe protocols." J Neurosci Methods 141(2): 171-198.

Michlovitz, S., et al. (1988). "Ice and High Voltage Pulsed Stimulation in Treatment of Acute Lateral Ankle Sprains." Journal of Orthopaedic \& Sports Physical Therapy 9(9): 301-304.

Miller, J. M. and R. C. de Guzman (2018). "PABA Release from Chitosan-PCL with Induced Electric Current." bioRxiv: 377481. 
Minassian, K., et al. (2012). "Neuromodulation of lower limb motor control in restorative neurology." Clin Neurol Neurosurg 114(5): 489-497.

Minhas, P., et al. (2010). "Electrodes for high-definition transcutaneous DC stimulation for applications in drug delivery and electrotherapy, including tDCS." J Neurosci Methods 190(2): 188-197.

Mongkolkitikul, S., et al. (2018). "Electrically controlled release of ibuprofen from conductive poly(3-methoxydiphenylamine)/crosslinked pectin hydrogel." Eur J Pharm Sci 112: 20-27.

Murdan, S. (2003). "Electro-responsive drug delivery from hydrogels." Journal of Controlled Release 92(1-2): 1-17.

Myllymaa, S., et al. (2012). "New disposable forehead electrode set with excellent signal quality and imaging compatibility." Journal of Neuroscience Methods 215: 103-109.

Naficy, S., et al. (2009). "Modulated release of dexamethasone from chitosan-carbon nanotube films." Sensors and Actuators A: Physical 155(1): 120-124.

Naficy, S., et al. (2012). "Electrically Conductive, Tough Hydrogels with pH Sensitivity." Chemistry of Materials 24(17): 3425-3433.

Nagamine, K., et al. (2016). "Totally shape-conformable electrode/hydrogel composite for on-skin electrophysiological measurements." Sensors and Actuators B: Chemical 237: 49-53.

Niamlang, S., et al. (2011). "Electrically Controlled Aloe-Vera Extraction Release from Poly Acrylamide Hydrogel." Energy Procedia 9: 468-473. 
Niamlang, S., et al. (2013). "Controlled Aloin Release from Crosslinked Polyacrylamide Hydrogels: Effects of Mesh Size, Electric Field Strength and a Conductive Polymer." Materials (Basel) 6(10): 4787-4800.

Niamlang, S. and A. Sirivat (2009). "Electrically controlled release of salicylic acid from poly(p-phenylene vinylene)/polyacrylamide hydrogels." Int J Pharm 371(1-2): 126-133.

Nunez, P. L., et al. (1994). "A theoretical and experimental study of high resolution EEG based on surface Laplacians and cortical imaging." Electroencephalography and Clinical Neurophysiology 90(1): 40-57.

O'Grady, M. L., et al. (2010). "Optimization of electroactive hydrogel actuators." ACS Appl Mater Interfaces 2(2): 343-346.

Organization, W. H. (2017). "Epilepsy fact sheet."

Osada, Y., et al. (1992). "A polymer gel with electrically driven motility." Nature 355: 242.

Paffi, A., et al. (2015). "Numerical characterization of intraoperative and chronic electrodes in deep brain stimulation." Front Comput Neurosci 9: 2.

Pairatwachapun, S., et al. (2016). "Controlled release of acetylsalicylic acid from polythiophene/carrageenan hydrogel via electrical stimulation." Carbohydr Polym 137: 214-221.

Pan, L., et al. (2012). "Hierarchical nanostructured conducting polymer hydrogel with high electrochemical activity." Proc Natl Acad Sci U S A 109(24): 9287-9292.

Pancrazio, J. J. (2008). "Neural interfaces at the nanoscale." Nanomedicine (Lond) 3(6): 823-830. 
Paradee, N. and A. Sirivat (2014). "Electrically controlled release of benzoic acid from poly(3,4-ethylenedioxythiophene)/alginate matrix: effect of conductive poly(3,4-ethylenedioxythiophene) morphology." J Phys Chem B 118(31): 9263-9271.

Paradee, N., et al. (2012). "Effects of crosslinking ratio, model drugs, and electric field strength on electrically controlled release for alginate-based hydrogel." J Mater Sci Mater Med 23(4): 999-1010.

Park, S. A., et al. (2013). "Evaluation of feature extraction methods for EEG-based brain-computer interfaces in terms of robustness to slight changes in electrode locations." Med Biol Eng Comput 51(5): 571-579.

Patra, A., et al. (2015). "Metal Free Conducting PEDOS, PEDOT, and Their Analogues via an Unusual Bromine-Catalyzed Polymerization." Macromolecules 48(24): 8760-8764.

Pawlaczyk, M., et al. (2013). "Age-dependent biomechanical properties of the skin." Postepy Dermatol Alergol 30(5): 302-306.

Pérez-Martínez, C. J., et al. (2016). "Electroconductive nanocomposite hydrogel for pulsatile drug release." Reactive and Functional Polymers 100: 12-17.

Pezaris, J. S. and E. N. Eskandar (2009). "Getting signals into the brain: visual prosthetics through thalamic microstimulation." Neurosurg Focus 27(1): E6.

Pilate, F., et al. (2014). "Design of Multistimuli-Responsive Shape-Memory Polymer Materials by Reactive Extrusion." Chemistry of Materials 26(20): 5860-5867. Polikov, V. S., et al. (2005). "Response of brain tissue to chronically implanted neural electrodes." J Neurosci Methods 148(1): 1-18. 
Prats-Boluda, G., et al. (2011). "Active concentric ring electrode for non-invasive detection of intestinal myoelectric signals." Med Eng Phys 33(4): 446-455.

Prats-Boluda, G., et al. (2012). "Active flexible concentric ring electrode for noninvasive surface bioelectrical recordings." Measurement Science and Technology 23(12).

Prausnitz, M. R., et al. (1993). "Electroporation of mammalian skin: a mechanism to enhance transdermal drug delivery." Proceedings of the National Academy of Sciences 90(22): 10504-10508.

Qu, J., et al. (2017). "pH-responsive self-healing injectable hydrogel based on Ncarboxyethyl chitosan for hepatocellular carcinoma therapy." Acta Biomater 58: $168-180$.

Qu, J., et al. (2018). "Injectable antibacterial conductive hydrogels with dual response to an electric field and $\mathrm{pH}$ for localized "smart" drug release." Acta Biomater 72: 55-69.

Ramanathan, S. and L. H. Block (2001). "The use of chitosan gels as matrices for electrically-modulated drug delivery." Journal of Controlled Release 70(1-2): $109-123$.

Ren, Y., et al. (2017). "Injectable hydrogel based on quaternized chitosan, gelatin and dopamine as localized drug delivery system to treat Parkinson's disease." Int J Biol Macromol 105(Pt 1): 1079-1087.

Richardson-Burns, S. M., et al. (2007). "Polymerization of the conducting polymer poly(3,4-ethylenedioxythiophene) (PEDOT) around living neural cells." Biomaterials 28(8): 1539-1552. 
Rols, M. P. (2006). "Electropermeabilization, a physical method for the delivery of therapeutic molecules into cells." Biochim Biophys Acta 1758(3): 423-428.

Rongies, W., et al. (2018). "Physiotherapy in postinfection injury to cranial nerves III, IV, and VI: a case study." Am J Phys Med Rehabil.

Rossmann, C., et al. (2017). "Temperature sensitive liposomes combined with thermal ablation: Effects of duration and timing of heating in mathematical models and in vivo." PLoS One 12(6): e0179131.

Rousche, P. J. and R. A. Normann (1998). "Chronic recording capability of the Utah Intracortical Electrode Array in cat sensory cortex." Journal of Neuroscience Methods 82(1): 1-15.

Rousche, P. J. and R. A. Normann (1999). "Chronic intracortical microstimulation (ICMS) of cat sensory cortex using the Utah intracortical electrode array." IEEE Transactions on Rehabilitation Engineering 7(1): 56-68.

Salisbury, D. B., et al. (2016). "Brain-computer interface for individuals after spinal cord injury." Rehabil Psychol 61(4): 435-441.

Sangwan, W., et al. (2016). "Electrically responsive materials based on polycarbazole/sodium alginate hydrogel blend for soft and flexible actuator application." Carbohydr Polym 151: 213-222.

Santini Jr, J. T., et al. (1999). "A controlled-release microchip." Nature 397: 335.

Saracino, G. A., et al. (2013). "Nanomaterials design and tests for neural tissue engineering." Chem Soc Rev 42(1): 225-262. 
Sasaki, M., et al. (2014). "Highly conductive stretchable and biocompatible electrodehydrogel hybrids for advanced tissue engineering." Adv Healthc Mater 3(11): 1919-1927.

Schachter, S. C., et al. (2009). "Advances in the Application of Technology to Epilepsy: The CIMIT/NIO Epilepsy Innovation Summit." Epilepsy \& Behavior 16(1): 3-46.

Schmidt, G., et al. (2014). "Electrochemotherapy in Breast Cancer: A Review of References." Geburtshilfe Frauenheilkd 74(6): 557-562.

Schoch, S. F., et al. (2017). "Dysregulation of Sleep Behavioral States in Narcolepsy." Sleep.

Sekine, S., et al. (2010). "Conducting polymer electrodes printed on hydrogel." J Am Chem Soc 132(38): 13174-13175.

Sersa, G. and D. Miklavcic (2008). "Electrochemotherapy of tumours." J Vis Exp(22).

Sershen, S. and J. West (2002). "Implantable, polymeric systems for modulated drug delivery." Advanced Drug Delivery Reviews 54(9): 1225-1235.

Servant, A., et al. (2014). "Graphene-based electroresponsive scaffolds as polymeric implants for on-demand drug delivery." Adv Healthc Mater 3(8): 1334-1343.

Servant, A., et al. (2013). "Electroresponsive polymer-carbon nanotube hydrogel hybrids for pulsatile drug delivery in vivo." Adv Healthc Mater 2(6): 806-811.

Sharifzadeh, G. and H. Hosseinkhani (2017). "Biomolecule-Responsive Hydrogels in Medicine." Adv Healthc Mater 6(24).

Shi, X., et al. (2015). "Dual stimulus responsive drug release under the interaction of $\mathrm{pH}$ value and pulsatile electric field for a bacterial cellulose/sodium 
alginate/multi-walled carbon nanotube hybrid hydrogel." RSC Advances 5(52): $41820-41829$.

Shi, Z., et al. (2014). "Double network bacterial cellulose hydrogel to build a biologydevice interface." Nanoscale 6(2): 970-977.

Shiga, T. and T. Kurauchi (1990). "Deformation of polyelectrolyte gels under the influence of electric field." Journal of Applied Polymer Science 39(1112): 2305-2320.

Silva, R. R., et al. (2015). "Dielectric Behavior of Alginate-Based Hydrogel Containing Neomycin-Loaded Lipid Nanovesicles under Influence of Electrical Potentials." Journal of the Brazilian Chemical Society.

Sittiwong, J., et al. (2012). "Electric field-controlled benzoic acid and sulphanilamide delivery from poly(vinyl alcohol) hydrogel." AAPS PharmSciTech 13(4): $1407-1415$.

Snyder, A. R., et al. (2010). "The influence of high-voltage electrical stimulation on edema formation after acute injury: a systematic review." J Sport Rehabil 19(4): 436-451.

Soundarajan, V. and W. G. Besio (2005). "Simulated comparison of disc and concentric electrode maps during atrial arrhythmias." IJBEM 7(1): 217-220.

Spiller, K. L., et al. (2014). "The role of macrophage phenotype in vascularization of tissue engineering scaffolds." Biomaterials 35(15): 4477-4488. 
Spiller, K. L., et al. (2015). "Sequential delivery of immunomodulatory cytokines to facilitate the M1-to-M2 transition of macrophages and enhance vascularization of bone scaffolds." Biomaterials 37: 194-207.

Spizzirri, U. G., et al. (2013). "Spherical gelatin/CNTs hybrid microgels as electroresponsive drug delivery systems." Int J Pharm 448(1): 115-122.

Stafstrom, C. E. and L. Carmant (2015). "Seizures and epilepsy: an overview for neuroscientists." Cold Spring Harb Perspect Med 5(6).

Staples, N. A., et al. (2017). "Conductive Hydrogel Electrodes for Delivery of LongTerm High Frequency Pulses." Front Neurosci 11: 748.

Sun, J. Y., et al. (2012). "Highly stretchable and tough hydrogels." Nature 489(7414): 133-136.

Sutani, K., et al. (2001). "The synthesis and the electric-responsiveness of hydrogels entrapping natural polyelectrolyte." Radiation Physics and Chemistry 61(1): 49-54.

Sutter, R., et al. (2013). "Clinical and imaging correlates of EEG patterns in hospitalized patients with encephalopathy." J Neurol 260(4): 1087-1098.

Tallgren, P., et al. (2005). "Evaluation of commercially available electrodes and gels for recording of slow EEG potentials." Clin Neurophysiol 116(4): 799-806.

Tamber, M. S. and J. M. Mountz (2012). "Advances in the diagnosis and treatment of epilepsy." Semin Nucl Med 42(6): 371-386.

Tao, Y., et al. (2005). "Polyacrylamide hydrogels with trapped sulfonated polyaniline." European Polymer Journal 41(6): 1342-1349. 
Teplan, M. (2002). "Fundamentals of EEG measurements." MEASUREMENT

\section{SCIENTIFIC REVIEW 2.}

Tessa, L. L., et al. (2015). "Field Reliability Estimation for Cochlear Implants." IEEE Trans Biomed Eng 62(8): 2062-2069.

Thakral, G., et al. (2013). "Electrical stimulation to accelerate wound healing." Diabet Foot Ankle 4.

Tilley, D. M., et al. (2015). "A continuous spinal cord stimulation model attenuates pain-related behavior in vivo following induction of a peripheral nerve injury." Neuromodulation 18(3): 171-176; discussion 176.

Tolaymat, A., et al. (2015). "Diagnosis and management of childhood epilepsy." Curr Probl Pediatr Adolesc Health Care 45(1): 3-17.

Tolouei, A. E., et al. (2018). "A Magnetically Responsive Biomaterial System for Flexibly Regulating the Duration between Pro- and Anti-Inflammatory Cytokine Deliveries." Adv Healthc Mater 7(12): e1800227.

Tomer, R., et al. (1995). "Electrically controlled release of macromolecules from cross-linked hyaluronic acid hydrogels." Journal of Controlled Release 33(3): 405-413.

Torkaman, G. (2014). "Electrical Stimulation of Wound Healing: A Review of Animal Experimental Evidence." Adv Wound Care (New Rochelle) 3(2): 202-218.

Tungkavet, T., et al. (2015). "Electromechanical properties of multi-walled carbon nanotube/gelatin hydrogel composites: effects of aspect ratios, electric field, and temperature." Mater Sci Eng C Mater Biol Appl 46: 281-289. 
Tungkavet, T., et al. (2015). "Graphene/gelatin hydrogel composites with high storage modulus sensitivity for using as electroactive actuator: Effects of surface area and electric field strength." Polymer 70: 242-251.

Uluturk, C. and N. Alemdar (2018). "Electroconductive 3D polymeric network production by using polyaniline/chitosan-based hydrogel." Carbohydr Polym 193: 307-315.

Uppalapati, D., et al. (2016). "Conducting polymers with defined micro- or nanostructures for drug delivery." Biomaterials 111: 149-162.

Usta, A. and R. Asmatulu (2016). "Synthesis and Analysis of Electrically Sensitive Hydrogels Incorporated with Cancer Drugs." Journal of Pharmaceutics \& Drug Delivery Research 5(2).

Wang, K., et al. (2017). "Stretchable Dry Electrodes with Concentric Ring Geometry for Enhancing Spatial Resolution in Electrophysiology." Adv Healthc Mater 6(19).

Wang, L., et al. (2018). "Tough, adhesive, self-healable and transparent ionically conductive zwitterionic nanocomposite hydrogels as skin strain sensors." ACS Appl Mater Interfaces.

Wang, L., et al. (2012). "Design and fabrication of a biodegradable, covalently crosslinked shape-memory alginate scaffold for cell and growth factor delivery." Tissue Eng Part A 18(19-20): 2000-2007.

Wang, L. L. and J. A. Burdick (2017). "Engineered Hydrogels for Local and Sustained Delivery of RNA-Interference Therapies." Adv Healthc Mater 6(1). 
Watanabe, Y., et al. (2017). "Blood-brain barrier derangement after electrical brain stimulation." Journal of neurology and neuromedicin 2(10): 1-5.

Weissenfels, A., et al. (2018). "Effects of whole-body electromyostimulation on chronic nonspecific low back pain in adults: a randomized controlled study." J Pain Res 11: 1949-1957.

Whelan, C. D., et al. (2018). "Structural brain abnormalities in the common epilepsies assessed in a worldwide ENIGMA study." Brain 141(2): 391-408.

WHO (2006). Neurological disorder: public health challenges. Geneva, Switzerland, World Health Organization.

Williams, C. S., et al. (2016). "Baseline EEG abnormalities in mild traumatic brain injury from the BIMA study." Undersea Hyperb Med 43(5): 521-530.

Williams, N. R. and M. S. Okun (2013). "Deep brain stimulation (DBS) at the interface of neurology and psychiatry." J Clin Invest 123(11): 4546-4556.

Wilson, A. N. and A. Guiseppi-Elie (2013). "Bioresponsive hydrogels." Adv Healthc Mater 2(4): 520-532.

Wilson, B. S. and M. F. Dorman (2008). "Cochlear implants: a remarkable past and a brilliant future." Hear Res 242(1-2): 3-21.

Wu, L., et al. (2015). "Electrically pulsatile responsive drug delivery platform for treatment of Alzheimer's disease." Nano Research 8(7): 2400-2414.

Wu, Q., et al. (2017). "A robust, highly stretchable supramolecular polymer conductive hydrogel with self-healability and thermo-processability." Sci Rep 7: 41566. 
Wu, Y., et al. (2016). "Fabrication of conductive gelatin methacrylate-polyaniline hydrogels." Acta Biomater 33: 122-130.

Wu, Y., et al. (2014). "Injectable Electroactive Hydrogels Formed via Host-Guest Interactions." ACS Macro Letters 3(11): 1145-1150.

Xi, J., et al. (2014). "Electrophoretic particle guidance significantly enhances olfactory drug delivery: a feasibility study." PLoS One 9(1): e86593.

Xiang, Y. and D. Chen (2007). "Preparation of a novel pH-responsive silver nanoparticle/poly(HEMA-PEGMA-MAA) composite hydrogel." European Polymer Journal 43(10): 4178-4187.

Xiao, Y., et al. (2012). "An effective approach for the fabrication of reinforced composite hydrogel engineered with SWNTs, polypyrrole and PEGDA hydrogel." Journal of Materials Chemistry 22(16).

Yamamoto, T. (2010). "Molecular assembly and properties of polythiophenes." NPG Asia Materials 2(2): 54-60.

Yan, F., et al. (2017). "Tumor-penetrating Peptide-integrated Thermally Sensitive Liposomal Doxorubicin Enhances Efficacy of Radiofrequency Ablation in Liver Tumors." Radiology 285(2): 462-471.

Yan, T., et al. (2018). "Cochlear implantation in patients with ossified cochleas." Am J Otolaryngol.

Yang, Y. and J. B. F. N. Engberts (2000). "Stimuli response of polysoap hydrogels in aqueous solution and DC electric fields." Colloids and Surfaces A: Physicochemical and Engineering Aspects 169(1-3): 85-94. 
Ye-Lin, Y., et al. (2013). "Tripolar Flexible Concentric Ring Electrode Printed with Inkjet Technology for ECG Recording." In: Roa Romero L. (eds) XIII Mediterranean Conference on Medical and Biological Engineering and Computing 2013 41(IFMBE Proceedings): 1579-1582.

Yi, Y. T., et al. (2015). "Programmable and on-demand drug release using electrical stimulation." Biomicrofluidics 9(2): 022401.

Ying, X., et al. (2014). "Angiopep-Conjugated Electro-Responsive Hydrogel Nanoparticles: Therapeutic Potential for Epilepsy." Angewandte Chemie 126(46): $12644-12648$.

Yokoi, Y., et al. (2017). "Transcranial Direct Current Stimulation in Depression and Psychosis: A Systematic Review." Clin EEG Neurosci: 1550059417732247.

Yoshida, R., et al. (2006). "Maskless microfabrication of thermosensitive gels using a microscope and application to a controlled release microchip." Lab Chip 6(10): 1384-1386.

Yu, J. M., et al. (2009). "EEG spectral changes underlying BOLD responses contralateral to spikes in patients with focal epilepsy." Epilepsia 50(7): 18041809.

Yuk, S. H., et al. (1992). "Electric current-sensitive drug delivery systems using sodium alginate/polyacrylic acid composites." Pharmaceutical Research 09(7): 955-957.

Yun, Y. H., et al. (2015). "Controlled Drug Delivery: Historical perspective for the next generation." J Control Release 219: 2-7. 
Zeng, F. G., et al. (2008). "Cochlear implants: system design, integration, and evaluation." IEEE Rev Biomed Eng 1: 115-142.

Zhang, D., et al. (2015). "Electroactive hybrid hydrogel: Toward a smart coating for neural electrodes." Journal of Bioactive and Compatible Polymers 30(6): 600616.

Zhao, Y., et al. (2018). "Microgel-Enhanced Double Network Hydrogel Electrode with High Conductivity and Stability for Intrinsically Stretchable and Flexible All-Gel-State Supercapacitor." ACS Appl Mater Interfaces 10(23): 1932319330.

Zhao, Y., et al. (2013). "3D nanostructured conductive polymer hydrogels for highperformance electrochemical devices." Energy \& Environmental Science $6(10)$.

Zhou, X., et al. (2002). "Numerical simulation of the steady-state deformation of a smart hydrogel under an external electric field." Smart Materials and Structures 11(3): 459-467.

Zubler, F., et al. (2017). "EEG synchronization measures are early outcome predictors in comatose patients after cardiac arrest." Clin Neurophysiol 128(4): 635-642. 